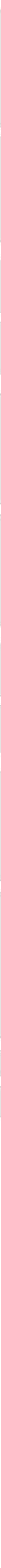

Elasticity and fracture

of

soft network materials 


\section{Propositions}

1. The classical view of crack propagation from defects does not apply in most biological networks.

(this thesis)

2. The fracture behaviour of polymer double networks is dominated by enthalpic stretching of polymer chains.

(this thesis)

3. The versatile cellular machinery demonstrates that there is also plenty of room in the middle for technological development.

4. Search engines bias the direction of academic research.

5. Scientific progress slows down if no space is provided, both literally and figuratively, for unguided discussions.

6. Copy-pasting the complete package of expectations from science to expectations from individual scientists, increases the stress experienced by researchers.

7. Student satisfaction is being misused as an indicator of educational quality.

8. Investing in new train tracks will hamper the transition to a sustainable mobility system in Europe.

Propositions belonging to the thesis, entitled

Dealing with stress

Elasticity and fracture of soft network materials

Justin Tauber

Wageningen, 22 October 2021 


\title{
Dealing with stress
}

\author{
Elasticity and fracture \\ of soft network materials
}




\section{Thesis committee}

\section{Promotor}

Prof. Dr J. van der Gucht

Professor of Physical Chemistry and Soft Matter

Wageningen University \& Research

\section{Co-promotor}

Dr S. Dussi

Harvard John A. Paulson School of Engineering and Applied Sciences

Harvard University, Cambridge, US

\section{Other members}

Prof. Dr J.L. van Leeuwen, Wageningen University \& Research

Dr E. Zaccarelli, CNR-ISC, Rome, Italy

Prof. Dr C. Storm, Eindhoven University of Technology

Dr B.P. Tighe, TU Delft 


\title{
Dealing with stress
}

\author{
Elasticity and fracture \\ of soft network materials
}

\author{
Justin Tauber
}

Thesis

submitted in fulfilment of the requirements for the degree of doctor at Wageningen University

by the authority of the Rector Magnificus,

Prof. Dr A.P. J. Mol,

in the presence of the

Thesis Committee appointed by the Academic Board,

to be defended in public

on Friday 22 October 2021

at 4 p.m. in the Aula. 
Justin Tauber

Dealing with stress - Elasticity and fracture of soft network materials 244 pages

$\mathrm{PhD}$ thesis, Wageningen University, Wageningen, The Netherlands (2021) With references, with summary in English

ISBN: 978-94-6395-946-9

DOI: https://doi.org/10.18174/552128 


\section{Contents}

1 General introduction................................................. 1

\section{Elasticity and fracture of elastic networks}

2 Fracture of athermal elastic networks ................................ 29

3 The role of temperature in the rigidity-controlled fracture of elastic networks 55

\section{Elasticity and fracture of composite networks}

4 Stress management in composite biopolymer networks ................. 95

5 Microscopic insights into the fracture of double elastic networks .......... 131

6 Sharing the load: stress redistribution governs fracture of polymer double

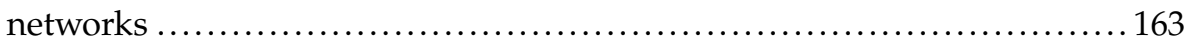

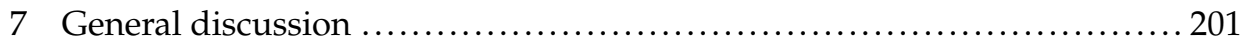

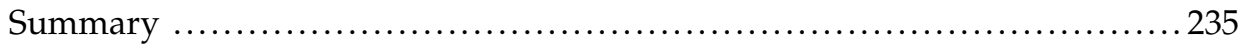

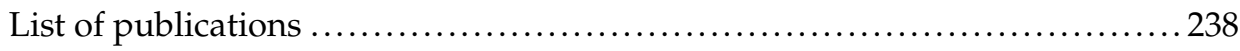

Acknowledgements .................................................... 239

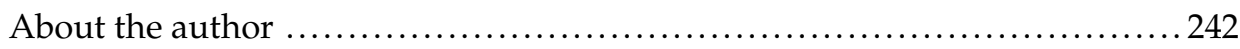

Overview of completed training activities ................................ 243 



\section{Chapter 1}

\section{General introduction}




\subsection{Why should you be interested in the fracture of soft network materials?}

When a material breaks this can be impractical, annoying or right out dangerous. Great care is taken every day to avoid the accumulation of damage in materials, but time has shown, again and again, that we can not prevent it. In this thesis, we will discuss the fracture of soft network materials. In contrast to stiff materials, these materials can be stretched significantly before any damage starts to accumulate. Furthermore, all these materials are networks at some level; therefore, the accumulation of damage occurs via the degradation of these network structures. Due to their high deformability prior to rupture, soft network materials have found a unique place in technology, especially since the discovery of rubber. Traditionally, these materials are used to make tires or to seal joints (Figure 1.1(a)), but more and more high deformability is used as a feature in advanced technologies, such as soft robotics (Figure 1.1(b) ${ }^{1,2}$. Soft network materials also play a key role in biology: in multicellular organisms cells are embedded in an extracellular matrix and even inside cells several network structures can be found that provide structural support (Figure 1.1(c) and (d)) ${ }^{3}$.

In the end, the accumulation of damage is an unavoidable process in soft network materials as well. Based on where we find these materials, we can identify two methods to mitigate the effects of fracture: the engineering approach and the biological approach. In the engineering approach one tries to detect the accumulation of damage at an early stage ${ }^{4}$ or delay damage accumulation via material composition or methods of usage. Here the guiding philosophy is that the accumulation of damage unavoidably leads to loss of function. By contrast, from a biological point of view the accumulation of damage does not have to be a slippery slope towards loss of function. Damage can be repaired ${ }^{5}$ or mitigated by network reorganization ${ }^{6}$, a process that can lead to new functionality, with cell motility as the prime example ${ }^{7}$. Material scientists have come to cherish this optimistic perspective on damage accumulation, leading to the development of (bio-inspired) responsive and self-healing materials ${ }^{8-10}$.

Both approaches require an intimate knowledge of the nature of elasticity and damage accumulation in soft network materials. Classically, elasticity and fracture have been studied from a macroscopic point of view. As a result, the microscopic structure of the material only has a minor role to play in most theories for elasticity and fracture. Nevertheless, all types of catastrophic failure must start at the microscopic level. A microscopic explanation of fracture would therefore deepen our understanding of the mechanical response of soft network materials. This microscopic explanation is especially desired in the context of soft network 

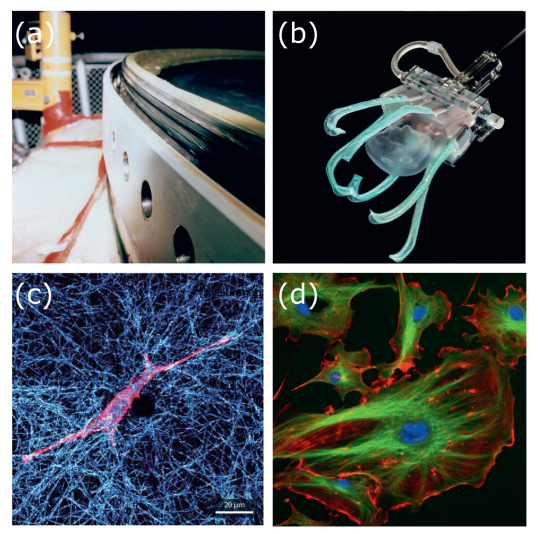

Figure 1.1. Examples of soft network materials in technology and biology. (a) Silicon o-rings, used to seal joints in the solid rocket booster of the space shuttle Challenger, lose deformability at sub-zero temperatures, decreasing the quality of the seal. Gas escaping through these seals likely caused the catastrophic explosion of the space shuttle in 1986 (source: NASA). (b) This soft robotic gripper is designed to capture fragile marine animals, like jellyfish, without causing damage to the organism. From Ref. [2]. Reprinted with permission from AAAS. (c) A cell embedded in the extracellular matrix. Reprinted from Ref. [3]. (d) The cytoskeleton (source: ImageJ).

materials for two reasons. Firstly, the microscopic structure of soft network materials is highly disordered, a property that has proven to influence the fracture of stiff materials to a great extent ${ }^{11}$. Secondly, for several soft network materials it has been shown that upon deformation the network undergoes significant rearrangements at the microscopic level ${ }^{12-14}$. These two phenomena suggest that the role of network structure cannot be overlooked.

The aim of this thesis is to explore the relation between network structure and fracture behaviour of soft network materials. Special attention is paid to composite networks, where interactions at the network level are expected to dominate both the elastic and fracture response. The presented work is numerical in nature, but the connection with experimental results plays a central role. In particular, we perform simulations in relation to fibre networks and polymer networks. We will show that networks deal with stress via the redistribution of load through the network, and that network structure dictates their elastic and fracture response.

\subsection{What are soft network materials?}

With the term "soft network materials" we describe a whole range of materials at once. Some of these you encounter every day (e.g. your bicycle tire), others 
(a)

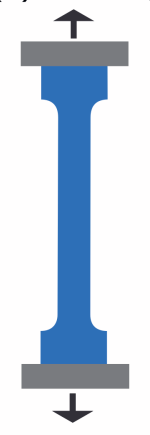

(b)

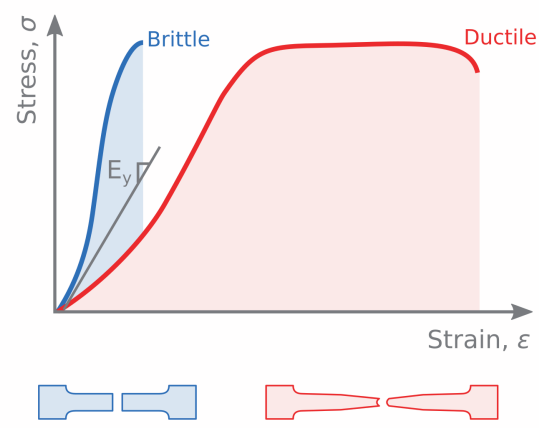

(c)

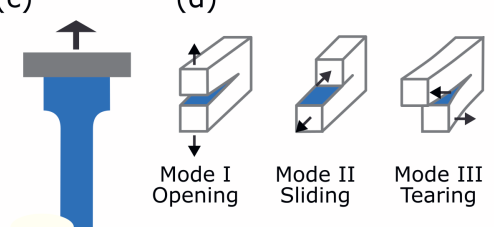

(e)

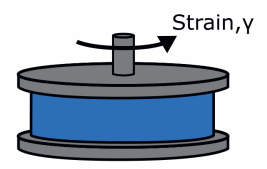

Figure 1.2. Quantifying the global mechanical response of soft materials. (a) Schematic of a tensile test on a dog bone shaped sample. (b) Schematic of the resulting stress-strain curve for brittle (blue) and ductile (red) fracture. The drawings underneath the plot represent the dogbones after failure. Young's Modulus $E_{y}$ is determined from the initial slope. Adapted from @Nicoguaro/CC-BY 4.0 (via Wikimedia Commons). (c) Schematic of a notched sample, used to measure the fracture energy for mode I fracture (crack opening). (d) The three fundamental modes of fracture: crack opening, sliding and tearing. (e) Schematic of a shear test used for very soft materials.

you rely on without knowing (in fact, the largest part of your body consists of soft materials) and some you might have never seen before. Below, we will introduce the soft network materials that we will discuss in this thesis.

To create a sense of how broad this class of materials really is, we provide a rough indication of their mechanical properties (summarized in Table 1.1). These mechanical properties are determined by measuring the force required to deform a sample of the material. To allow easy comparison between measurements, the measured force and corresponding deformation are rescaled to stress and strain, respectively. For example, for a tensile test, where the material is elongated as shown in Figure 1.2(a), the measured force is divided by the initial cross-sectional area to obtain the engineering stress $\sigma=F / A_{0}$ and the deformation is divided by the initial length of the sample to obtain the engineering strain $\epsilon=\left(L-L_{0}\right) / L_{0}$. From the resulting stress versus strain curve (Figure 1.2(b)) we can extract several useful properties, such as the initial resistance to deformation, also called Young's Modulus $E_{y}$, which is equal to the initial slope, and the fracture strength, the maximum load that the material can carry. Furthermore, the toughness of the sample can be quantified by measuring the energy required to expand a crack from a notch in the sample (Figure 1.2(c)), this value is called the fracture energy $\Gamma$. 
(a)

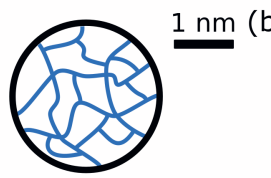

Elastomers

(e)

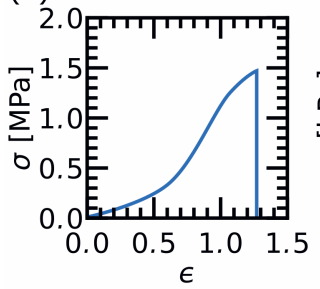

(b)

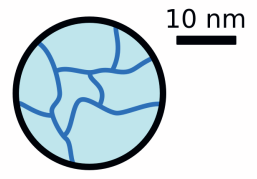

Hydrogels

(f)

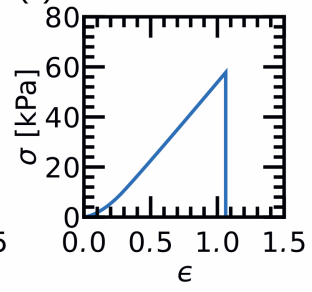

(c)

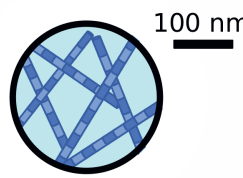

Fibre networks

(g)

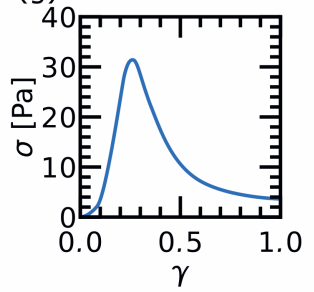

(d)

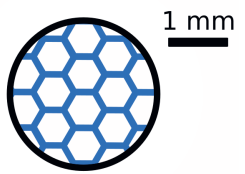

(h)

Macroscopic networks

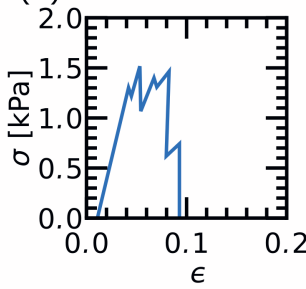

Figure 1.3. Failure response of networks over a range of length scales. (a-d) Schematic representation of (a) an elastomer ( $1 \mathrm{~nm})$, (b) a hydrogel $(10 \mathrm{~nm})$, (c) a fibre network (100 nm-1 $\mu \mathrm{m})$ and (d) a macroscopic network (e.g. metamaterials, $1 \mathrm{~mm}-1 \mathrm{~cm}$ ). (e-h) Fabricated examples of the mechanical behaviour. (e) Stress response to a tensile test on an elastomer, e.g. PDMS ${ }^{15}$. (f) Stress response to a tensile test on a hydrogel, e.g. tetra-PEG ${ }^{16}$. (g) Stress response to a shear test on a fibre network, e.g. collagen ${ }^{17}$. (h) Stress response to a tensile test on a macroscopic network, e.g. a laser-cut acrylic sheet ${ }^{18}$.

Elastomers - One of the most prevalent network materials are polymeric network materials consisting solely of flexible polymer strands interconnected by crosslinkers (Figure 1.3(a)), such as thermosetting rubbers and plastics. Because we are studying the role of network structure we are most interested in polymer networks above their glass temperature $T_{g}$, i.e. elastomers, where enthalpic interactions between chains are small and the polymers in between the crosslinkers behave as in a polymer melt. As a consequence, the majority of the interactions between polymers in the material takes place via the network ${ }^{19}$. Still some other interactions occur in elastomers as well, such as entanglements and molecular friction, which are known to influence the mechanical response before and during fracture $^{20}$. Because in elastomers the entire volume of the material is occupied by polymer chains, the material can be quite stiff with a Young's modulus ranging from 0.01 to $10 \mathrm{GPa}^{21}$ (for comparison, steel has a Young's modulus of $\sim 200 \mathrm{GPa}$ ). Their tensile fracture strength ranges from 1 to $50 \mathrm{MPa}^{15,21}$ and their mode I fracture energy from 50 to $1000 \mathrm{~J} \mathrm{~m}^{-2}$ (see Ref. [20]). In Figure 1.3(e) an example of a stress-strain curve is shown, revealing an abrupt fracture response after the maximum strength is reached. Prior to the peak a strain-softening regime can be seen, which is present in some elastomers ${ }^{15}$. Because elastomers combine high deformability prior to damage accumulation with a reasonably high stiffness, these 


\begin{tabular}{lllll}
\hline \hline Type & $\begin{array}{l}\text { Weight } \\
\text { fraction }\end{array}$ & $\begin{array}{l}\text { Linear } \\
\text { Modulus }\end{array}$ & Strength & $\begin{array}{l}\text { Fracture } \\
\text { energy }\end{array}$ \\
\hline Elastomers & $100 \%$ & $10^{4}-10^{7} \mathrm{~Pa}$ & $10^{6}-10^{7} \mathrm{~Pa}$ & $50-1000 \mathrm{~J} \mathrm{~m}^{-2}$ \\
Hydrogels & $10 \%$ & $10^{3}-10^{5} \mathrm{~Pa}$ & $10^{4}-10^{5} \mathrm{~Pa}$ & $0.5-10 \mathrm{~J} \mathrm{~m}^{-2}$ \\
Fibre networks & $0.5-5 \%$ & $10-100 \mathrm{~Pa}$ & $10-200 \mathrm{~Pa}$ & - \\
\hline
\end{tabular}

Table 1.1. Elastic and fracture properties of soft network materials.

materials are often used in an engineering context. To improve their mechanical properties, elastomers can be reinforced, for example by the introduction of filler particles (this is why rubber tires are black) and, more recently, by crosslinking additional polymer networks inside an existing elastomer as shown in Fig 1.4(a). The latter approach can even shift the typically abrupt or brittle fracture response of elastomer networks to a more gradual or ductile response (see Figure 1.2(b)), significantly extending the fracture regime and increasing the fracture energy from $50 \mathrm{~J} \mathrm{~m}^{-2}$ up to $5000 \mathrm{~J} \mathrm{~m}^{-2}{ }^{22}$

Hydrogels - Another class of polymeric network materials is that of polymer gels, which are polymer network materials embedded in a solvent, e.g. a polymer gel dissolved in water is called a hydrogel. Because the weight fraction of polymers is significantly lower in these materials (around $10 \%$ of the total weight), they are typically softer and weaker than elastomers. This also means a significant reduction in interactions between polymers below the network level, making these materials interesting candidates for the study of how network structure affects the failure behaviour. However, the presence of solvent also introduces new physical phenomena such as the swelling of polymer chains and a coupling between network deformation and fluid flow referred to as poroelasticity. Typically, their Young's modulus ranges from 1 to $100 \mathrm{kPa}^{22}$, their tensile fracture strength ranges from 10 to $100 \mathrm{kPa}^{23}$ and their mode I fracture energy is around $10 \mathrm{~J} \mathrm{~m}^{-2}$ (see Ref. [24]). An example of a stress-strain curve is shown in Figure 1.3(f). Due to their lower stiffness and high solvent content, these materials are often used in a biomedical context. However, single network hydrogels can be very sensitive to defects in the material. To prevent this problem a hydrogel can be reinforced, similar to elastomers, by crosslinking a second network inside the existing network, forming a double network with moduli from 0.1 to $1.0 \mathrm{MPa}^{25}$, fracture strengths of 1 to $10 \mathrm{MPa}^{25}$ and fracture energies of $10^{2}-10^{3} \mathrm{~J} \mathrm{~m}^{-2}$ comparable to unfilled elastomers $^{24}$. Also hydrogel double networks show a distinct ductile fracture response, significantly reducing the flaw-sensitivity (Figure 1.4(b)). 
Fibre networks - Besides networks made out of polymers, there are also networks composed of fibres such as actin and collagen (Figure 1.3(c)). These fibres are typically formed by the complexation of macromolecules. As a result, these fibres are thicker and longer than single stranded polymers, making it possible to observe these networks through a microscope. Also, the resulting networks are coarser with mesh sizes ranging from $1 \mu \mathrm{m}$ to $10 \mu \mathrm{m}^{12,26,27}$. Fibre networks are typically embedded in a solvent, just like polymeric gels. Again the mass fraction of the network is very low (ranging from $0.5 \%$ to $5 \%{ }^{26,28-30}$ ), so that most interactions take place via the network. Because these fibre networks are coarser than polymeric gels, it is easier for the solvent to move around the fibres, reducing (but not removing) poroelastic effects ${ }^{3,31}$. The nature of the crosslinks in fibre networks can vary widely, from linkages via flexible proteins to intricate branching and interweaving of fibre bundles. Interactions within these fibre networks can be highly dynamic. Due to the low fibre density, the linear stiffness of these materials is very low, making classical mechanical tests like extension extremely difficult. Therefore, elasticity and fracture behaviour of these materials is often tested under shear as shown schematically in Figure 1.2(e). Their linear stiffness, referred to as the shear modulus $G^{\prime}$, ranges from $10 \mathrm{~Pa}$ to $100 \mathrm{~Pa}^{26,30}$ and their strength varies from $10 \mathrm{~Pa}$ to $200 \mathrm{~Pa}^{28,32}$. Fracture energies are not well studied because of the extension problem. An example of a stress-strain curve is shown in Figure 1.3(g), which reveals a more gradual decay after the peak stress. Most examples of fibre networks can be found in a biological context, providing structural support both inside and outside of cells ${ }^{3}$. In fact, many of these biological structures, such as the cytoskeleton and the extracellular matrix can be considered as a composite as they consist of a mixture of several biological networks (Figure 1.1(d)) ${ }^{3}$. In addition, there are several examples of synthetic fibre networks ${ }^{33,34}$.

Macroscopic networks - So far we have considered materials in which we cannot see the structure with our naked eye. Even a small volume of those materials still contains enormous amounts of elements and crosslinkers. However, many networks exist that we can observe with our own eyes. Examples are textiles, fishing nets, and several metamaterials. Also for research purposes these well-defined macroscopic networks have been fabricated in order to study the fracture response ${ }^{37}$. The properties of these materials can vary widely, depending on what material the network is made from. But, in general, the macroscopic network is softer and more stretchable than the material in its bulk form. An example of a stress-strain curve for a single network is shown in Figure 1.3(h) and an example of a composite is shown in Figure 1.4(c). Because the number of elements in macroscopic networks is very low, the fracture of individual bonds is visible in the global stress-strain response. 
(a)

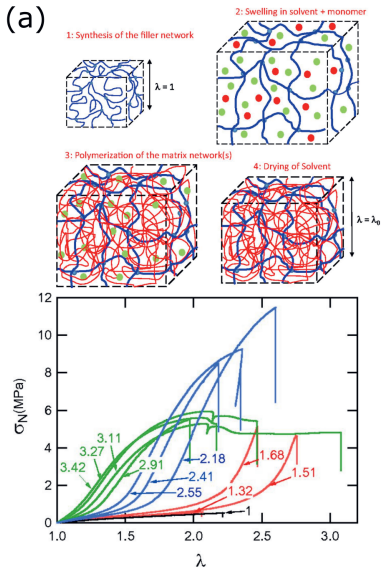

(b)

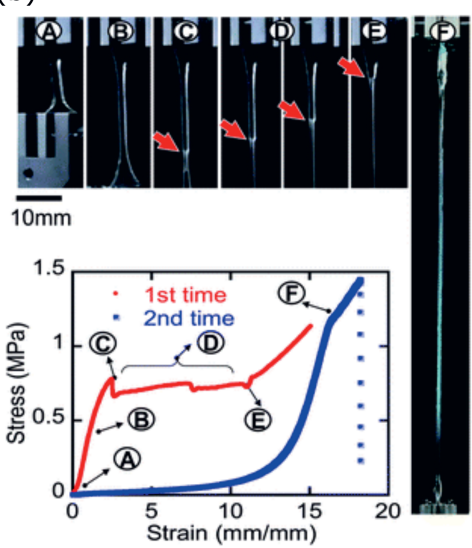

(C) Wood - Silicone rubber

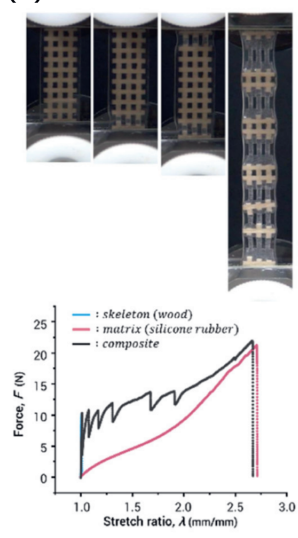

Figure 1.4. Examples of composite networks materials. (a) An elastomeric composite can be created by swelling a sacrifical network (blue) inside a matrix monomer, and later crosslinking these monomers in to a matrix network. The bottom panel shows the mechanical response (normal stress $\sigma_{N}$ versus stretch $\lambda$ ) upon uniaxial extension. As a function of the swelling ratio $\lambda_{0}$, the mechanical response can be shifted from a brittle (red) to a ductile response (green). The coloured numbers in the bottom panel indicate the $\lambda_{0}$ value corresponding to each curve. Reprinted from Ref. [35]. Copyright 2018 National Academy of Sciences. (b) A hydrogel composite network. At the start of the plateau in the stress-strain curve a neck appears in the material as demonstrated by the pictures. Reprinted from Ref. [25]. (c) A macroscopic composite. Adapted with permission from Ref. [36]. Copyright 2019 American Chemical Society.

\subsubsection{Fundamentals of network elasticity}

With these examples in mind, we can distinguish two main contributions to the mechanical response of a soft network material: the nature of the building blocks and the structure of the network.

The elastic response of the building blocks, the piece of polymer or fibre in between crosslinkers, stems from their resistance to deformation; therefore, the exact elastic response of these building blocks is highly dependent on the nature and size of the building block. In particular, the length of the building block $l_{c}$ compared to its persistence length $l_{p}$ plays an important role. The persistence length is a measure for the ease of undulating a polymer via thermal motions and is defined as $l_{p}=\kappa /\left(k_{\mathrm{B}} T\right)$ with $\kappa$ the bending rigidity and $k_{\mathrm{B}} T$ the thermal energy. If the structure in between crosslinks is very flexible or very long, the thermal fluctuations dominate the elastic response. For example, in polymers $\left(l_{c} \gg l_{p}\right.$, Figure 1.5(a)) and flexible fibres $\left(l_{c} \gtrsim l_{p}\right.$, Figure 1.5(b)) the initial response is governed by entropy, where the resistance to stretching is caused by a reduction 


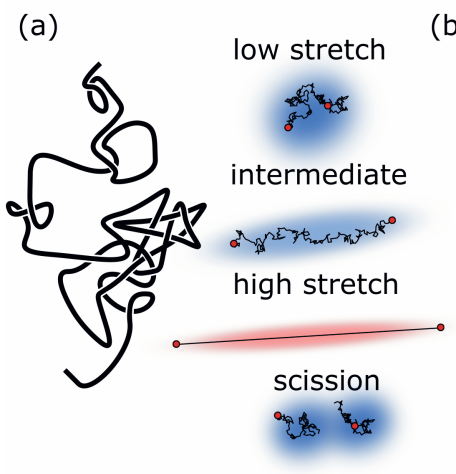

(b)

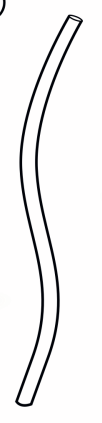

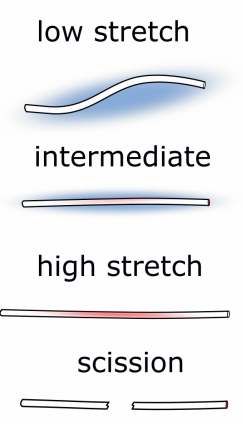

(c)

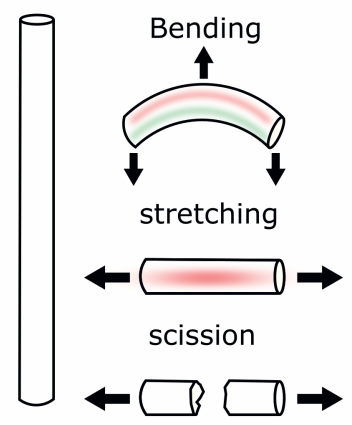

Figure 1.5. Building block elasticity. The elastic response of a building block depends on the nature of the building block and the extent or type of deformation. We distinguish between (a) flexible polymers, (b) semi-flexible polymers, and (c) stiff fibres. The resistance to deformation can be due to entropy (blue), enthalpic stretching (red) or enthalpic compression (green).

in accessible conformations (indicated in blue), followed by an enthalpic regime, where extension results in stretching of molecular bonds (indicated in red). Due to these different regimes the elastic response is highly non-linear. For stiffer or shorter fibres $\left(l_{c} \ll l_{p}\right.$, Figure 1.5(c)) the entropic effects are negligible, and the mechanical response is completely governed by the (non-)covalent interactions inside the fibre; these are also called athermal fibres. The elastic response of these fibres is closer to a linear response. What all these elements have in common is that they will break if they are stretched far enough, most often within an enthalpic regime. For flexible polymers the extension at rupture will be several times the initial distance between the crosslinkers, while for stiff fibres the extension at rupture might only be slightly larger than the initial distance.

Even though the origin of the material response is the elastic response of the building blocks, there is no one-to-one correspondence between the elasticity of a single building block and the mechanical response of an entire network. It is obvious that in a network not all building blocks are perfectly aligned with the applied deformation field, which is one factor that needs to be considered. More importantly, networks have the ability to rearrange if they are deformed to minimize the amount of load on the building blocks. These rearrangements are called non-affine, because they deviate from the global "affine" deformation that is applied. The consequences of these non-affine rearrangements at the network level can be surprising, such as the strain-stiffening response of collagen ${ }^{13}$ or the auxetic response of metamaterials ${ }^{38}$. How the distribution of load takes place is governed by the network structure, or specifically network rigidity, as we will explain in 


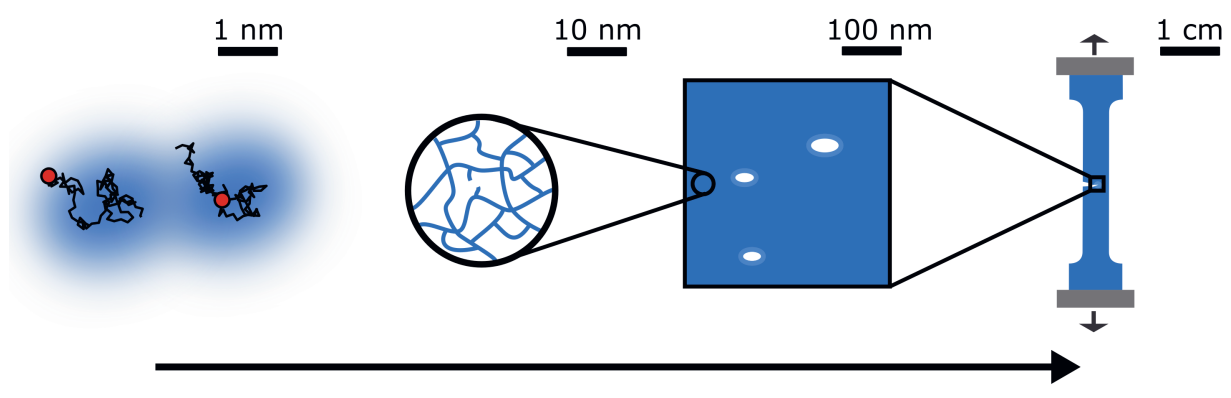

Figure 1.6. Bridging length scales. Fracture starts with the scission of a single chain, leading to accumulation of damage to the local network structure. Damage can occur in the bulk due to heterogeneity in the network structure or around small defects ${ }^{39}$. Eventually, a microcrack will nucleate and propagate to form a macroscopic crack.

more detail in Chapter 2. If these non-affine rearrangements occur, the distribution of tension in the network will be highly dependent on the network structure and therefore also the failure of building blocks can be expected to depend on the structure of the network. However, a clear picture of this relation between network structure and fracture is still lacking.

\subsection{Why is material fracture a difficult problem?}

The reason that the effect of network structure on material fracture is poorly understood, is the large separation in length scales between the first fracture event (breaking of a single chain) and the global failure caused by the evolution of macroscopic cracks as shown schematically in Figure 1.6. Capturing everything that happens in between these length scales in experiment, numerical models, or theory, is a challenge. In addition, there is a large separation in time scales: while the initial damage might accumulate slowly, the transition towards global failure often happens in the blink of an eye.

Also the network structure itself poses a problem. Typically, the intrinsic disorder provides little opportunity for simplification of the network structure, as is commonly done in studies on crystalline solids. In principle, this means that an accurate description of elasticity and fracture should involve all the components of the network.

Due to the disordered structure it is hard to predict when and where fracture will occur, especially because a full characterization of each and every part of the network is practically impossible. Nevertheless, we know that on average the fracture response between different samples of the same material is reproducible. 
(a)
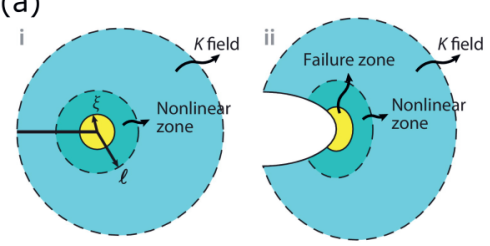

(b)

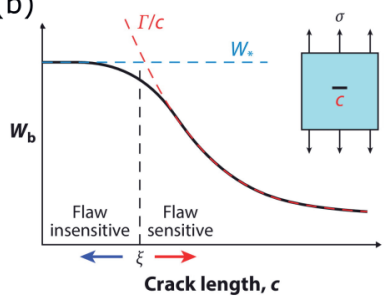

(c)

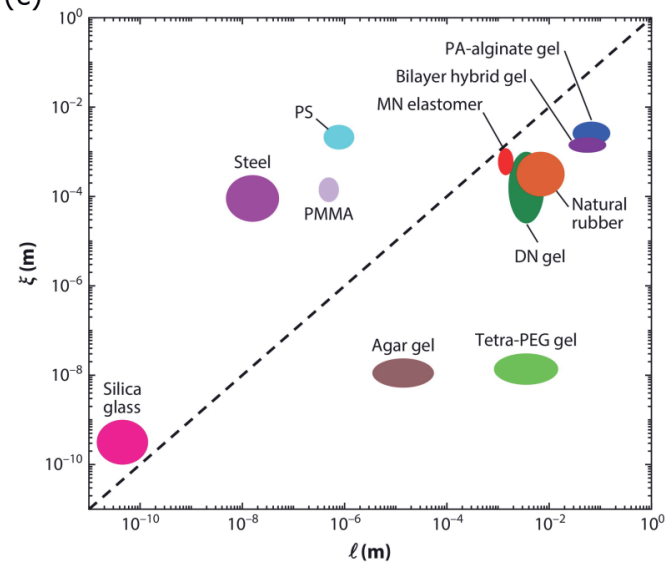

Figure 1.7. Length scales in continuum elasticity. (a) Around a crack tip two zones can be identified: the dissipative zone, where energy dissipates prior to expansion of the crack, and the non-linear zone, where non-linear elasticity dominates. The size of the largest defect in the material with respect to these lengths determines the fracture response. (b) In particular, if $\xi \ll c$ stress can easily concentrate at the tips of the defect, and thus the energy required for fracture will be flaw-sensitive $W_{b} \sim \Gamma / c$. However, if $\xi \gg c$ the fracture response will be flaw-insensitive and the fracture energy is dominated by $W^{* 15,40,41}$, the energy required for fracture if no defects are present. With this information and assuming that the size of the defect $c$ is significantly smaller than the size of the sample $L$, four types of materials can be identified: stiff brittle materials $(\ell \ll \xi \ll c)$, stiff ductile materials $(\ell \ll \xi \sim c)$, soft brittle materials $(\xi \ll \ell \ll c)$, and soft ductile materials $(\ell \gg \xi \sim c)$. (c) $\xi$ and $\ell$ for a wide range of materials. For all materials considered in this thesis $\xi<\ell$ (they lie below the dashed line). Reprinted with permission from Ref. [42].

Fracture can therefore be described as a statistical process. What makes fracture a tough statistical problem is that the fracture events are not independent: which bonds fail does not only depend on the initial network structure, but also on the evolution of damage and structural reorganizations in the network.

Despite these three challenges, there is great interest from science and technology to improve our microscopic understanding of fracture in soft network materials. In broad lines the (microscopic) fracture response takes place in three stages:

1. Damage accumulation - In the damage accumulation stage, damage develops homogeneously in the bulk of the material ${ }^{22}$. Whether this damage is caused by heterogeneity in the network structure or only occurs around larger heterogeneities arising from sample preparation is still unclear ${ }^{43,44}$. 
However, in some materials this type of damage can lead to significant softening, also called the Mullins effect ${ }^{22,45,46}$.

2. Crack nucleation - Subsequently, microscopic cracks will start to nucleate. The difference between these cracks and the damage accumulated in the first regime is that the cracks have a distinguishable crack surface. Alternatively, microscopic cracks can already be present, for example in the form of gas bubbles.

3. Crack propagation - Finally, one or several microscopic cracks will propagate to form macroscopic cracks until a crack spans the entire system, leading to catastrophic failure.

Little is known about the processes leading up to the propagation of a crack in soft materials and also the initial stage of crack propagation, where in principle many cracks can propagate at the same time, remains relatively unexplored. By contrast, a significant amount of experimental and theoretical research has been done on the propagation of cracks from defects in polymer networks based on an extension of linear elastic fracture mechanics (LEFM). We summarize these findings here, as they provide both a useful perspective on the process of fracture and a motivation for our research. The aim of these theories is to predict at which global stress a crack will propagate from a defect. For soft network materials, this requires detailed knowledge about the effect of large deformation on stress concentration at the crack tip. In particular, two length scales are introduced: the non-linear length scale $\ell$ and the dissipative length scale $\xi^{42}$. The first length scale is the distance from the crack tip over which the stress response is highly non-linear, which can be written as $\ell \sim \Gamma / E_{y}$. The second length scale is the distance over which stress concentration is smeared out due to dissipation processes $\xi \sim \Gamma / W_{*}$ with $W_{*}$ the critical energy per unit volume for material failure without a pre-existing crack. In soft materials $\ell$ and $\xi$ are typically a lot larger than the atomic length scales and the size of the building blocks $l_{c}$ as shown for several materials in Figure 1.7(c). How these length scales compare to the size of a typical defect with size $c$, determines the global fracture response. We are interested in two cases: flaw-sensitive soft brittle materials $(\xi \ll \ell \ll c)$, such as many hydrogels, and the flaw-insensitive soft ductile materials $(\ell \gg \xi \sim c)$, such as many double networks, for both of which $\ell \gg \xi$ and thus $W_{*} \gg E_{y}{ }^{42}$. Although these length scales are useful tools in classifying the fracture response of soft polymer networks, the microscopic damage processes that control these length scales are poorly understood. Furthermore, it is unclear if these ideas can be translated to the fibre networks and macroscopic networks introduced in Section 1.2. Bridging the gap between the current macroscopic understanding of fracture and the emerging picture of damage accumulation 
(a)

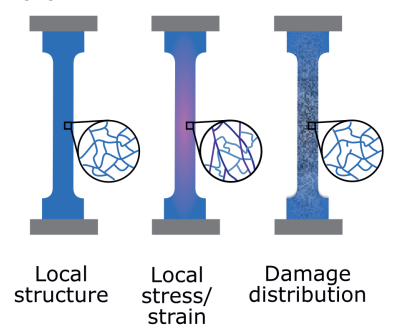

strain

Structural characterization

Live detection

Post-mortem analysis (b)
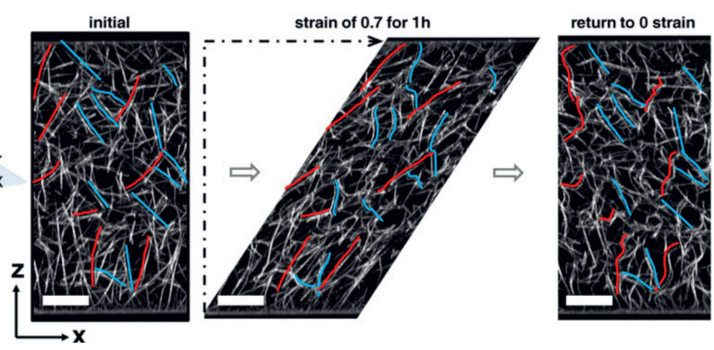

(c)
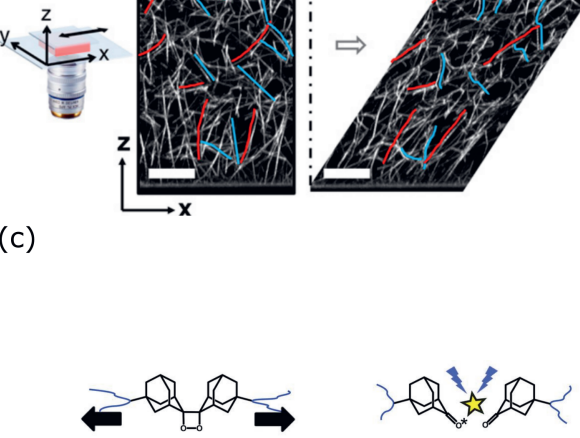

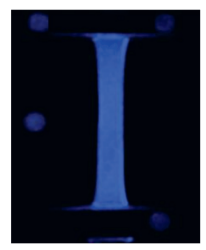

Figure 1.8. Experimental state-of-the-art. (a) Data of interest and the stages of the fracture experiment at which local information can be obtained. (b) Direct study of plastic events in fibrin networks using confocal microscopy. Reprinted from Ref. [28]. (c) Dioxetane mechanophores as a reporter for chain scission. The dioxetane mechanophore emits a photon upon bond scission, which can be detected with a camera, as is shown here for a strip of an elastomeric composite under uniaxial extension. From Ref. [22]. Reprinted with permission from AAAS.

at the microscopic level is one of the important motivations for this thesis. To start bridging this gap both state-of-the art experimental techniques and powerful simulation techniques are required that can capture the microscopic response to fracture.

\subsection{Experimental state-of-the-art}

A complete description of the microscopic failure process includes: local stress, local strain and damage accumulation (Figure 1.8(a)). Ideally, these data are acquired at the same time during the fracture process, so that the spatial and temporal correlation in the data can be studied. Realistically, this is not always possible, therefore there are also techniques that either study the structure of the network prior to deformation or post-mortem. Although temporal information is lost with these techniques, they do allow a higher spatial resolution as the acquisition time is no longer dependent on the fracture process. Here, we will discuss some of these experimental techniques to reflect the state-of-the-art and 
provide insight into the experimental data that is available on the microscopic damage process.

Which measurement technique works best depends highly on the material and the problem of interest (deformation mode, length scale and timescale). For fibre networks it is possible to study the microscopic fracture process using microscopy. In particular confocal laser scanning microscopy can be very helpful in this regard because it provides three-dimensional information on the fracture process ${ }^{12,28}$. A drawback of microscopy is that it allows to only study a small part of the sample; large rearrangements can cause the area of interest to move out of the field of view quickly. For hydrogels and elastomers microscopy is not so useful, at least not during the fracture process, because the network structure can not be resolved. To at least give some idea about the development of cracks in the nucleation process, small-angle scattering techniques such as SAXS and SANS can be used. Although these techniques can resolve features at and below the network level, the ensemble averaged nature of the data makes interpretation difficult ${ }^{47-49}$. Spatially resolved scattering techniques such as laser speckle imaging 9,50 , on the other hand, are very promising in studying fracture problems. Although this technique can not resolve the network structure, it provides information on dynamics for an impressive range of length and time scales. In a sense, this technique provides information on local strain. However, this technique is mostly useful for fracture phenomena where the global deformation is limited.

LSI already demonstrated that it is not always needed to completely resolve the network structure in order to study microscopic processes, as long as somehow the local response can be measured. One completely different trick to measure local response is to chemically modify the network components, so that they can visualize strain, stress or damage. Intriguing chemical designs, called mechanophores, do just that ${ }^{22,51-55}$. In particular, we can distinguish damage reporters ${ }^{22,55}$, like dioxetane (Fig. 1.8(c)) and merocyanine, from stress/strain reporters ${ }^{53,54}$, like spiropyran. A major challenge for these reporters is not only to introduce them into the networks, but also to quantify their output ${ }^{55}$.

Besides these techniques used to study material failure, we want to emphasize the importance of single molecule experiments ${ }^{56}$ (or single fibre experiments ${ }^{57}$ ), which are very helpful to understand the elasticity and fracture at the building block level.

\subsection{Modelling network elasticity and fracture}

As described earlier, network fracture is a problem with many facets. For a specific fracture problem it can be difficult to determine which factors are dominant in 
(a)

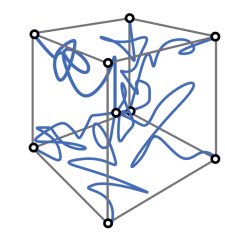

(e)

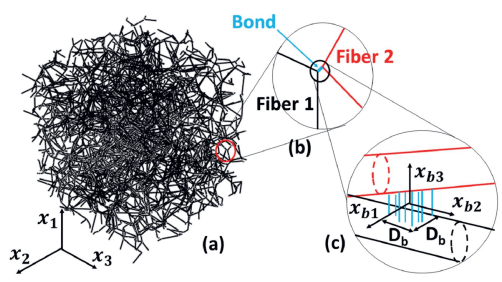

(b)

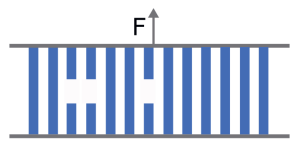

(f)
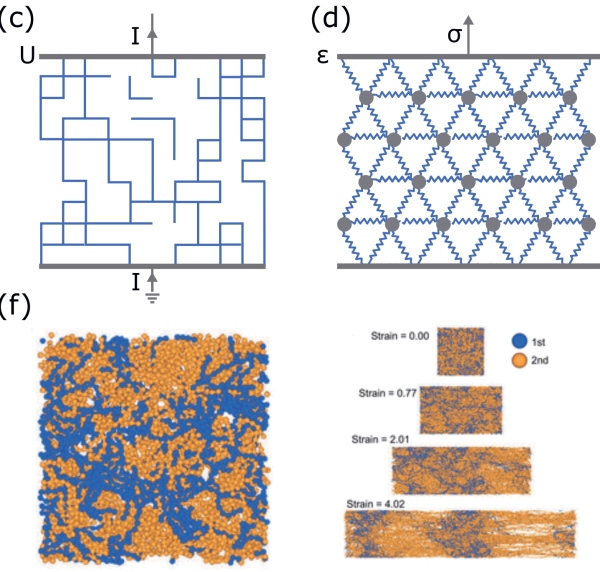

(d)

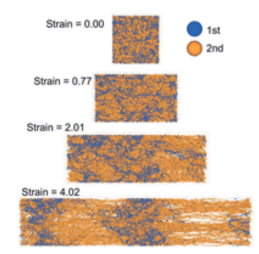

Figure 1.9. Modelling approaches. (a) Schematic of the Arruda-Boyce 8-chain model used to build constitutive models ${ }^{58}$. (b) Schematic of a fibre bundle model ${ }^{59}$. After bond breaking the load is redistributed over the remaining fibres according to a pre-defined load sharing rule. (c) Schematic of a diluted random fuse model. The current $I$ and voltage $U$ serve as analogues for stress $\sigma$ and strain $\epsilon$. (d) Schematic of a spring network model. Springs break if they exceed a pre-defined threshold. (e) Example of a fibre network model that includes bending interactions along fibres and a more detailed description of inter-fibre bonds. Reprinted from Ref. [60] with permission from Elsevier. (f) Example of Kremer-Grest type model for a polymer network. Each polymer consists out of several particles and each bond between these particles represents a Kuhn segment. Adapted with permission from Ref. [61]. Copyright 2018 American Chemical Society.

controlling the fracture response, especially because not all these factors can be controlled easily in experiment. Computer models can significantly speed up the process of identifying these dominant factors ${ }^{62}$. It is important to realize that these simulations are not meant to replace experimental research. The true power of these models is that they allow one to test the validity of the assumptions that are made, explicitly or implicitly, in the interpretation of experiments.

\subsubsection{Continuum models}

The crudest approach to model the mechanical response is to capture the elastic and the fracture response of a material in a constitutive law that describes the elastic and fracture response of a representative volume element in the material upon deformation ${ }^{44}$. Here representative means that a sufficiently large piece of the material is described, such that increasing its size does not significantly alter the mechanical response. In the context of soft network materials, this 
approach is most developed for polymer networks and especially elastomers. The description of the mechanical response can either be phenomenological ${ }^{63,64}$ or based on a microscopic description of elasticity (Figure 1.9(a) ${ }^{44,58}$. Typically, this micromechanical approach describes the evolution in the end-to-end distance of a set of polymers within the representative volume element, assuming that the deformation inside the volume element is completely affine (on average), i.e. follows the global deformation, and that the behaviour of each polymer is independent. Based on a description for the entropic elasticity of the individual chains ${ }^{65}$, a constitutive law for the deformation of the entire volume element can be constructed. The evolution of damage can also be described with a technique coined statistical damage mechanics ${ }^{44}$, where it is assumed that the initial configuration of polymer chains contains some distribution in the end-to-end distances and that chains break if their extension exceeds a certain threshold. The type of damage accumulation captured in this model is the diffuse damage that happens in the bulk prior to crack nucleation. On its own, these models can not capture the role of defects in material fracture; however, if they are implemented in finite element models ${ }^{66}$ it is possible to look into stress concentration around defects ${ }^{54}$ and necking ${ }^{67}$.

\subsubsection{Fibre bundle models}

The drawback of (most) constitutive models is that they do not describe any interaction between the building blocks in the network (in this case polymers). A cheap computational approach to study fracture as a function of this interaction is to use fibre bundle models. In these models the fracture of fibres that are arranged in a one-dimensional or two-dimension array is studied (Figure 1.9(b)). The fracture strength of each fibre is picked from a strength distribution and subsequently the fibre bundle is put under a load. If a fibre breaks, the load is redistributed according to a pre-defined load-sharing rule. Within these rules two limits can be identified, global load sharing, where the load carried by the broken fibre is redistributed equally over all remaining fibres and local load sharing, where the load is only distributed over the neighbouring fibres. Although these fibres are not arranged into a network, which makes the translation to real network materials challenging, this load sharing rule could act as a proxy for how stress is managed within a network ${ }^{59}$.

\subsubsection{Spring and fibre network models}

An alternative to this approach is to explicitly build a network structure, where the sharing of load emerges from the nature of the elements, e.g. linear springs, 
and the associated physics. The stress distribution is found by solving a system of equations. For simplicity, most of the work in this area has been based on scalar versions of networks: networks of electrical fuses (Figure 1.9(c)). This approach relies on the analogy between the stress distribution in spring networks and current flow in fuse networks ${ }^{68}$. The latter is a simpler problem to solve because the functions are scalar, i.e. rearrangements in the network do not affect the result.

Checks of this fuse approach show that indeed for networks with a high amount of disorder in the rupture threshold the scalar problem does provide similar results to the tensorial variant of a network with mechanical springs (Figure 1.9(d) ${ }^{69,70}$. However, in recent years it has become clear that the tensorial approach to elasticity does actually give rise to a qualitatively different fracture response, especially for networks that are diluted down to the Maxwell isostatic point ${ }^{37,71}$. This difference lies in the importance of rigidity in the elastic response of these networks, that has also been shown to be highly relevant in the (non-)linear elasticity of biological fiber networks ${ }^{13,72}$. These spring models will play a central role in this thesis, therefore a detailed discussion will follow in Chapter 2. Several extensions of these models exist wherein also other elastic contributions are considered, such as models for fibre networks that include tension, bending and torsion ${ }^{73}$. Furthermore, additional mechanisms of bond scission can be implemented as depicted in Figure 1.9(e) ${ }^{60}$.

Note that all the models above (by design) do not take into account time. Essentially, they describe elasticity and fracture under quasi-static conditions, where (thermal) fluctuations are not important. To some extent the effect of these dynamics, i.e. kinetics, can be added by implementing something like a Gillespie algorithm ${ }^{74}$. However, such an approach would assume that there is no coupling in the dynamics of two consecutively breaking bonds. Alternatively, the spring networks can be embedded in any type of dynamics simulation where, for example, Newton's equations are solved (see Chapter 3).

\subsubsection{Coarse-grained polymer network models}

The models described thus far do capture the network structure, but below the network structure they do not describe any details. Not even excluded volume interactions are considered, so that elements pass through each other without any consequence. As we discussed previously, temperature plays an important role in the (linear) elasticity of soft polymer network materials. To capture this effect, a more detailed description of the elements is needed, as is done in the widely-used Kremer-Grest model ${ }^{75}$. Networks are created using various protocols and instead of single springs, the polymers are represented by a string of bonded beads, each 


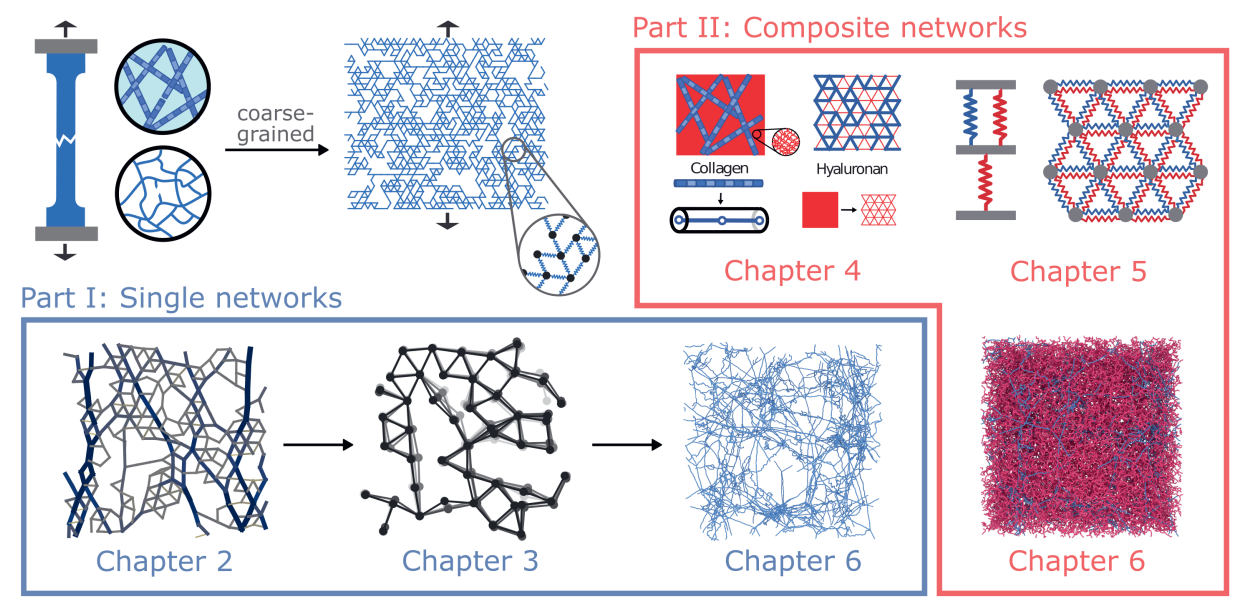

Figure 1.10. Graphical abstract for this thesis. We investigate the microscopic processes that are involved in the elasticity and fracture of soft network materials, such as fibre networks and elastomers. In particular, we want to know how the network controls the elastic and fracture response. Therefore, we study several coarse-grained models that focus on network structure. In part I we study single networks while we shift our focus to composites in part II.

representing a Kuhn segment of the polymer. Because of this design, entropic effects are included explicitly in the model and also topological constraint between different polymers are captured (see Chapter 6). Although more accurate, these models are computationally expensive so that the networks simulated necessarily are small, making the study of defect effects difficult.

\subsection{Scope and outline}

The aim of this thesis is to explore the relation between network structure and fracture behaviour of soft network materials. As outlined in this introduction, the microscopic fracture mechanism both prior and during the propagation of macroscopic cracks remains unclear. Therefore, we investigate how networks deal with stress in both soft brittle and soft ductile materials, such as double network materials. To this end, we study the mechanical response of several minimal network models summarized in Figure 1.10. As all damage in soft network materials is preceded by large deformation, we also pay attention to the relation between (non-)linear elasticity and network structure. Furthermore, we make comparisons with experimental work where possible. We have divided the following research chapters into two parts. 
Part I: elasticity and fracture of elastic networks - We start with a minimal central-force elastic model for network elasticity and fracture in the athermal regime in Chapter 2. We show that the mechanical response of subisostatic networks, the class most soft network materials fall in due to their low connectivity, is qualitatively different from hyperstatic networks, which are typically used to study fracture of stiff materials. We demonstrate that the fracture response of these networks is highly dependent on the connectivity of the network, which is reflected both in the global and microscopic response. Finally, we also demonstrate that this minimal model can be used to study the fracture response of collagen networks. Then, in Chapter 3, we take the same networks, but instead of minimizing the energy we now add thermal fluctuations via a dynamics model. We show that temperature acts as a stabilizing field, in agreement with literature results based on Monte Carlo simulations. From there we show that this stabilizing effect is also present in the non-linear regime and that there is a coupling between rigidity and temperature, which determines how fracture occurs.

Part II: elasticity and fracture of composite networks - In Chapter 4 we first look into the elasticity of collagen networks embedded in the polysaccharide hyaluronan, essentially a double network of biopolymers. We show that hyaluronan works as a stabilizing field in the linear regime, providing additional stiffness. In the non-linear regime, the presence of hyaluronan shifts the non-linear response of the collagen network due to pre-stress at the network level. Moving to fracture, we dive into the transition from brittle to ductile fracture in composite networks. This brittle-to-ductile transition has been demonstrated in networks at several length scales. In Chapter $\mathbf{5}$ we study a random spring network model as a minimal model for double network fracture. We show that in this minimal model the brittleto-ductile transition is controlled by a force balance between the two networks. Furthermore, we demonstrate that network structure and disorder do affect the fracture mechanisms during the transition, but that in this model the location of the transition is unaffected. This chapter is followed by Chapter $\mathbf{6}$ where we make a more detailed study of fracture in swollen polymer double networks using a Kremer-Grest type model. While in Chapter 5 the interaction between the two networks was pre-defined, the interaction between the two networks in Chapter 6 only takes place via topological constraints. As a result, we find that the fracture response shows two distinct regimes: one controlled by stress redistribution within the first network and one controlled by stress redistribution between the two networks. Our investigation of the stress distribution process reveals that also in more realistic polymer networks the microscopic fracture response is controlled by network structure. 
These chapters answer some questions about the relation between network structure and the fracture response. However, more question arose during this project. In the general discussion we will look back at the research to distil common trends, discuss implications and present exciting future research directions.

\section{List of symbols}

\begin{tabular}{|c|c|}
\hline Symbol & Description \\
\hline$A_{0}$ & Initial cross-sectional area perpendicular to axis of deformation \\
\hline$c$ & Crack length \\
\hline$E_{y}$ & Young's modulus \\
\hline$F$ & Force applied along the axis of deformation \\
\hline$G^{\prime}$ & Elastic shear modulus or storage modulus \\
\hline$k_{\mathrm{B}}$ & Boltzmann constant \\
\hline$l_{c}$ & Length of the building block (e.g. polymer) between crosslinks \\
\hline$l_{p}$ & Persistence length \\
\hline$\ell$ & Non-linear length scale (fracture mechanics) \\
\hline$L$ & Length of sample along axis of deformation \\
\hline$L_{0}$ & Initial length of sample along axis of deformation \\
\hline$T$ & Temperature \\
\hline$T_{g}$ & Glass temperature \\
\hline W & $\begin{array}{l}\text { Work of extension or energy required for fracture (area under the } \\
\text { stress-strain curve) }\end{array}$ \\
\hline$W_{*}$ & $\begin{array}{l}\text { Critical energy per unit volume for material failure without a } \\
\text { pre-existing crack }\end{array}$ \\
\hline$\gamma$ & Shear strain \\
\hline$\Gamma$ & Fracture energy \\
\hline$\epsilon$ & Uniaxial or normal strain defined as $\left(L-L_{0}\right) / L_{0}$ \\
\hline$\kappa$ & Bending rigidity \\
\hline$\lambda$ & Stretch ratio defined as $L / L_{0}$ \\
\hline$\xi$ & Dissipative length scale (fracture mechanics) \\
\hline$\sigma$ & Stress \\
\hline$\sigma_{N}$ & Normal stress (stress measured upon uniaxial deformation) \\
\hline
\end{tabular}




\section{References}

[1] E. W. Hawkes, L. H. Blumenschein, J. D. Greer, A. M. Okamura, A soft robot that navigates its environment through growth. Science Robotics 2, eaan3028 (2017).

[2] N. R. Sinatra, C. B. Teeple, D. M. Vogt, K. K. Parker, D. F. Gruber, R. J. Wood, Ultragentle manipulation of delicate structures using a soft robotic gripper. Science Robotics 4, eaax5425 (2019).

[3] F. Burla, Y. Mulla, B. E. Vos, A. Aufderhorst-Roberts, G. H. Koenderink, From mechanical resilience to active material properties in biopolymer networks. Nature Reviews Physics 1, 249-263 (2019).

[4] P. Pissis, G. Georgousis, C. Pandis, P. Georgiopoulos, A. Kyritsis, E. Kontou, M. Micusik, K. Czanikova, M. Omastova, Strain and Damage Sensing in Polymer Composites and Nanocomposites with Conducting Fillers. Procedia Engineering 114, 590-597 (2015).

[5] A. Aher, D. Rai, L. Schaedel, J. Gaillard, K. John, Q. Liu, M. Altelaar, L. Blanchoin, M. Thery, A. Akhmanova, CLASP Mediates Microtubule Repair by Restricting Lattice Damage and Regulating Tubulin Incorporation. Current Biology 30, 2175-2183 (2020).

[6] R. S. Kadzik, K. E. Homa, D. R. Kovar, F-Actin Cytoskeleton Network Self-Organization through Competition and Cooperation. Annual Review of Cell and Developmental Biology 36, 35-60 (2020).

[7] A. Lambrechts, M. Van Troys, C. Ampe, The actin cytoskeleton in normal and pathological cell motility. International Journal of Biochemistry and Cell Biology 36, 1890-1909 (2004).

[8] S. Utrera-Barrios, R. Verdejo, M. A. López-Manchado, M. Hernández Santana, Evolution of self-healing elastomers, from extrinsic to combined intrinsic mechanisms: A review. Materials Horizons 7, 2882-2902 (2020).

[9] H. M. van der Kooij, A. Susa, S. J. García, S. van der Zwaag, J. Sprakel, Imaging the Molecular Motions of Autonomous Repair in a Self-Healing Polymer. Advanced Materials 29, 1701017 (2017).

[10] D. L. Taylor, M. in het Panhuis, Self-Healing Hydrogels. Advanced Materials 28, 9060-9093 (2016).

[11] D. Bonamy, E. Bouchaud, Failure of heterogeneous materials: A dynamic phase transition? Physics Reports 498, 1-44 (2011).

[12] R. C. Arevalo, P. Kumar, J. S. Urbach, D. L. Blair, Stress heterogeneities in sheared type-I collagen networks revealed by boundary stress microscopy. PLOS ONE 10, 0118021 (2015).

[13] A. Sharma, A. J. Licup, K. A. Jansen, R. Rens, M. Sheinman, G. H. Koenderink, F. C. Mackintosh, Strain-controlled criticality governs the nonlinear mechanics of fibre networks. Nature Physics 12, 584-587 (2016).

[14] D. E. Hanson, J. L. Barber, A new paradigm for the molecular basis of rubber elasticity. Contemporary Physics 56, 319-337 (2015). 
[15] T. K. Kim, J. K. Kim, O. C. Jeong, Measurement of nonlinear mechanical properties of PDMS elastomer. Microelectronic Engineering 88, 1982-1985 (2011).

[16] Y. Akagi, T. Katashima, Y. Katsumoto, K. Fujii, T. Matsunaga, U. I. Chung, M. Shibayama, T. Sakai, Examination of the theories of rubber elasticity using an ideal polymer network. Macromolecules 44, 5817-5821 (2011).

[17] F. Burla, J. Tauber, S. Dussi, J. van der Gucht, G. H. Koenderink, Stress management in composite biopolymer networks. Nature Physics 15, 549-553 (2019).

[18] E. Berthier, J. E. Kollmer, S. E. Henkes, K. Liu, J. M. Schwarz, K. E. Daniels, Rigidity percolation control of the brittle-ductile transition in disordered networks. Physical Review Materials 3, 075602 (2019).

[19] L. R. Treloar, The elasticity and related properties of rubbers. Reports on Progress in Physics 36, 755-826 (1973).

[20] C. Creton, 50th Anniversary Perspective: Networks and Gels: Soft but Dynamic and Tough. Macromolecules 50, 8297-8316 (2017).

[21] R. A. Shanks, I. Kong, Advances in Elastomers I, no. March (Springer, Berlin, Heidelberg, 2013), pp. 11-45, first edn.

[22] E. Ducrot, Y. Chen, M. Bulters, R. P. Sijbesma, C. Creton, Toughening elastomers with sacrificial bonds and watching them break. Science 344, 186-189 (2014).

[23] J. Zhang, C. R. Daubert, E. A. Foegeding, Characterization of polyacrylamide gels as an elastic model for food gels. Rheologica Acta 44, 622-630 (2005).

[24] R. Long, C.-Y. Hui, Fracture toughness of hydrogels: measurement and interpretation. Soft Matter 12, 8069-8086 (2016).

[25] J. P. Gong, Why are double network hydrogels so tough? Soft Matter 6, 2583-2590 (2010).

[26] F. C. MacKintosh, J. Kas, P. A. Janmey, Elasticity of biopolymer networks. Physical Review Letters 75, 4425-4429 (1995).

[27] J. Xia, L. H. Cai, H. Wu, F. C. MacKintosh, D. A. Weitz, Anomalous mechanics of Zn2+-modified fibrin networks. Proceedings of the National Academy of Sciences of the United States of America 118, e2020541118 (2021).

[28] S. Munster, L. M. Jawerth, B. a. Leslie, J. I. Weitz, B. Fabry, D. a. Weitz, Strain history dependence of the nonlinear stress response of fibrin and collagen networks. Proceedings of the National Academy of Sciences of the United States of America 110, 12197-12202 (2013).

[29] J. Zhu, L. J. Kaufman, Collagen i self-assembly: Revealing the developing structures that generate turbidity. Biophysical Journal 106, 1822-1831 (2014).

[30] K. A. Jansen, A. J. Licup, A. Sharma, R. Rens, F. C. MacKintosh, G. H. Koenderink, The Role of Network Architecture in Collagen Mechanics. Biophysical Journal 114, 2665-2678 (2018). 
[31] M. T. Punter, B. E. Vos, B. M. Mulder, G. H. Koenderink, Poroelasticity of (bio)polymer networks during compression: Theory and experiment. Soft Matter 16, 1298-1305 (2020).

[32] F. Burla, S. Dussi, C. Martinez-Torres, J. Tauber, J. van der Gucht, G. H. Koenderink, Connectivity and plasticity determine collagen network fracture. Proceedings of the National Academy of Sciences of the United States of America 117, 8326-8334 (2020).

[33] S. Fleming, R. V. Ulijn, Design of nanostructures based on aromatic peptide amphiphiles. Chemical Society Reviews 43, 8150-8177 (2014).

[34] P. de Almeida, M. Jaspers, S. Vaessen, O. Tagit, G. Portale, A. E. Rowan, P. H. Kouwer, Cytoskeletal stiffening in synthetic hydrogels. Nature Communications 10, 609 (2019).

[35] P. Millereau, E. Ducrot, J. M. Clough, M. E. Wiseman, H. R. Brown, R. P. Sijbesma, C. Creton, Mechanics of elastomeric molecular composites. Proceedings of the National Academy of Sciences of the United States of America 115, 9110-9115 (2018).

[36] D. R. King, T. Okumura, R. Takahashi, T. Kurokawa, J. P. Gong, Macroscale Double Networks: Design Criteria for Optimizing Strength and Toughness. ACS Applied Materials and Interfaces 11, 35343-35353 (2019).

[37] M. M. Driscoll, B. G. G. Chen, T. H. Beuman, S. Ulrich, S. R. Nagel, V. Vitelli, The role of rigidity in controlling material failure. Proceedings of the National Academy of Sciences of the United States of America 113, 10813-10817 (2016).

[38] D. R. Reid, N. Pashine, J. M. Wozniak, H. M. Jaeger, A. J. Liu, S. R. Nagel, J. J. De Pablo, Auxetic metamaterials from disordered networks. Proceedings of the National Academy of Sciences of the United States of America 115, E1384-E1390 (2018).

[39] J. Y. Kim, Z. Liu, B. M. Weon, T. Cohen, C. Y. Hui, E. R. Dufresne, R. W. Style, Extreme cavity expansion in soft solids: Damage without fracture. Science Advances 6, eaaz0418 (2020).

[40] C. Chen, Z. Wang, Z. Suo, Flaw sensitivity of highly stretchable materials. Extreme Mechanics Letters 10, 50-57 (2017).

[41] C. Yang, T. Yin, Z. Suo, Polyacrylamide hydrogels. I. Network imperfection. Journal of the Mechanics and Physics of Solids 131, 43-55 (2019).

[42] R. Long, C. Y. Hui, J. P. Gong, E. Bouchbinder, The Fracture of Highly Deformable Soft Materials: A Tale of Two Length Scales. Annual Review of Condensed Matter Physics 12, 71-94 (2021).

[43] H. J. Qi, M. C. Boyce, Constitutive model for stretch-induced softening of the stressstretch behavior of elastomeric materials. Journal of the Mechanics and Physics of Solids 52, 2187-2205 (2004).

[44] F. J. Vernerey, R. Brighenti, R. Long, T. Shen, Statistical Damage Mechanics of Polymer Networks. Macromolecules 51, 6609-6622 (2018). 
[45] L. Mullins, N. R. Tobin, Stress softening in rubber vulcanizates. Part I. Use of a strain amplification factor to describe the elastic behavior of filler-reinforced vulcanized rubber. Journal of Applied Polymer Science 9, 2993-3009 (1965).

[46] G. Marckmann, E. Verron, L. Gornet, G. Chagnon, P. Charrier, P. Fort, A theory of network alteration for the Mullins effect. Journal of the Mechanics and Physics of Solids 50, 2011-2028 (2002).

[47] T. Tominaga, V. R. Tirumala, E. K. Lin, J. P. Gong, H. Furukawa, Y. Osada, W. li Wu, The molecular origin of enhanced toughness in double-network hydrogels: A neutron scattering study. Polymer 48, 7449-7454 (2007).

[48] E. Ducrot, H. Montes, C. Creton, Structure of Tough Multiple Network Elastomers by Small Angle Neutron Scattering. Macromolecules 48, 7945-7952 (2015).

[49] K. Fukao, T. Nakajima, T. Nonoyama, T. Kurokawa, T. Kawai, J. P. Gong, Effect of Relative Strength of Two Networks on the Internal Fracture Process of Double Network Hydrogels As Revealed by in Situ Small-Angle X-ray Scattering. Macromolecules 53, 1154-1163 (2020).

[50] H. M. Van Der Kooij, S. Dussi, G. T. Van De Kerkhof, R. A. Frijns, J. Van Der Gucht, J. Sprakel, Laser Speckle Strain Imaging reveals the origin of delayed fracture in a soft solid. Science Advances 4, eaar1926 (2018).

[51] M. Stratigaki, R. Göstl, Methods for Exerting and Sensing Force in Polymer Materials Using Mechanophores. ChemPlusChem 85, 1095-1103 (2020).

[52] Y. Chen, G. Mellot, D. Van Luijk, C. Creton, R. P. Sijbesma, Mechanochemical tools for polymer materials. Chemical Society Reviews 50, 4100-4140 (2021).

[53] T. van de Laar, H. Schuurman, P. van der Scheer, J. Maarten van Doorn, J. van der Gucht, J. Sprakel, Light from Within: Sensing Weak Strains and FemtoNewton Forces in Single Molecules. Chem 4, 269-284 (2018).

[54] Y. Chen, C. Joshua Yeh, Y. Qi, R. Long, C. Creton, From force-responsive molecules to quantifying and mapping stresses in soft materials. Science Advances 6, eaaz5093 (2020).

[55] J. Slootman, V. Waltz, C. J. Yeh, C. Baumann, R. Göstl, J. Comtet, C. Creton, Quantifying Rate-and Temperature-Dependent Molecular Damage in Elastomer Fracture. Physical Review X 10, 41045 (2020).

[56] S. Wang, S. Panyukov, M. Rubinstein, S. L. Craig, Quantitative Adjustment to the Molecular Energy Parameter in the Lake-Thomas Theory of Polymer Fracture Energy. Macromolecules 52, 2772-2777 (2019).

[57] S. M. Iqbal, D. Deska-Gauthier, L. Kreplak, Assessing collagen fibrils molecular damage after a single stretch-release cycle. Soft Matter 15, 6237-6246 (2019).

[58] E. M. Arruda, M. C. Boyce, A three-dimensional constitutive model for the large stretch behavior of rubber elastic materials. Journal of the Mechanics and Physics of Solids 41, 389-412 (1993). 
[59] S. Roy, S. Biswas, P. Ray, Modes of failure in disordered solids. Physical Review E 96, 063003 (2017).

[60] S. Deogekar, R. C. Picu, On the strength of random fiber networks. Journal of the Mechanics and Physics of Solids 116, 1-16 (2018).

[61] Y. Higuchi, K. Saito, T. Sakai, J. P. Gong, M. Kubo, Fracture Process of Double-Network Gels by Coarse-Grained Molecular Dynamics Simulation. Macromolecules 51, 3075-3087 (2018).

[62] J. Lei, Z. Li, S. Xu, Z. Liu, Recent advances of hydrogel network models for studies on mechanical behaviors. Acta Mechanica Sinica 37, 367-386 (2021).

[63] A. N. Gent, A new constitutive relation for rubber. Rubber Chemistry and Technology 69, 59-61 (1996).

[64] M. Bacca, C. Creton, R. M. McMeeking, A Model for the Mullins Effect in Multinetwork Elastomers. Journal of Applied Mechanics 84, 121009 (2017).

[65] R. Jedynak, Approximation of the inverse Langevin function revisited. Rheologica Acta 54, 29-39 (2015).

[66] W. Li, T. J. Delaney, X. Jiao, R. Samulyak, C. Lu, Finite element model for brittle fracture and fragmentation. Procedia Computer Science 80, 245-256 (2016).

[67] V. Morovati, M. A. Saadat, R. Dargazany, Necking of double-network gels: Constitutive modeling with microstructural insight. Physical Review E 102, 062501 (2020).

[68] P. M. Duxbury, P. L. Leath, P. D. Beale, Breakdown properties of quenched random systems: The random-fuse network. Physical Review B 36, 367-380 (1987).

[69] P. K. V. Nukala, S. Zapperi, S. Åimunović, Statistical properties of fracture in a random spring model. Physical Review E 71, 066106 (2005).

[70] P. D. Beale, D. J. Srolovitz, Elastic fracture in random materials. Physical Review B 37, 5500-5507 (1988).

[71] L. Zhang, D. Z. Rocklin, L. M. Sander, X. Mao, Fiber networks below the isostatic point: Fracture without stress concentration. Physical Review Materials 1, 052602 (2017).

[72] C. P. Broedersz, X. Mao, T. C. Lubensky, F. C. Mackintosh, Criticality and isostaticity in fibre networks. Nature Physics 7, 983-988 (2011).

[73] R. C. Picu, Mechanics of random fiber networks - A review. Soft Matter 7, 6768-6785 (2011).

[74] D. T. Gillespie, A general method for numerically simulating the stochastic time evolution of coupled chemical reactions. Journal of Computational Physics 22, 403-434 (1976).

[75] K. Kremer, G. S. Grest, Dynamics of entangled linear polymer melts: A moleculardynamics simulation. The Journal of Chemical Physics 92, 5057-5086 (1990). 



\section{PART I}

Elasticity and fracture of elastic networks 



\section{Chapter 2}

\section{Fracture of athermal elastic networks}

Although the term "soft network materials" describes a wide range of material types, they all share a disordered network structure at some level. In this chapter we introduce a minimal network model, based on a randomly diluted triangular lattice of Hookean springs, that allows us to study the role of network structure in the elastic and fracture response of soft network materials. We find that the rigidity dependence of the elastic response is reflected in the fracture response and can be tuned via the fraction of bonds $p$ remaining after dilution and the rupture threshold $\lambda$ of individual bonds. Furthermore, we find a system size dependency in the fracture response, as is expected for fracture phenomena. Finally, we demonstrate, based on experimental shear measurements on collagen networks, that this athermal central-force elastic model can be used to interpret the fracture response of real soft network materials.

\section{Simone Dussi, Justin Tauber \& Jasper van der Gucht}

"Athermal fracture of elastic networks: How rigidity challenges the unavoidable size-induced brittleness"

Physical Review Letters 124, 018002 (2020)

\section{Federica Burla*, Simone Dussi*, Cristina Martinez-Torres, Justin Tauber, Jasper van der Gucht \& Gijsje H. Koenderink}

"Connectivity and plasticity determine collagen network fracture"

Proceedings of the National Academy of Sciences 117, 8326-8334 (2020)

*these authors contributed equally. 


\subsection{Introduction}

Soft network materials exist in numerous forms, ranging from polymer networks, such as PDMS elastomers, to fibre networks, such as collagen. Also in colloidal gels an underlying network structure can be identified, and several metamaterials and textiles can be considered network materials as well. Many of these materials share a highly disordered microstructure and can undergo large deformations before damage becomes visible at the macroscopic level. From theory ${ }^{1,2}$ and experimental work ${ }^{3}$ we know that the combination of this disordered network structure and large deformations leads to heterogeneity in stress at the microscopic level, i.e. the load is not shared equally over all parts of the network.

We want to understand how these characteristic properties of soft network materials control their fracture behaviour. However, due to the wide range of length and time scales associated with the process of network fracture, it is challenging to conduct a systematic study both in experiments and in system specific simulation studies. To mitigate this challenge, we propose to make strong assumptions on the elastic and fracture response of building blocks, leading to a highly coarse-grained model of soft network materials that operates at the mesoscale.

In particular, we investigate the structure dependent fracture response of an athermal central-force elastic model inspired by a model for the (non-)linear elasticity of athermal fibre networks ${ }^{4-6}$. We highlight the role of network size on the fracture response and show how size scaling can give a deeper insight in the role of stress concentration in the fracture of networks. Finally, we demonstrate how this framework can be used to interpret experimental results on the fracture of collagen networks.

\subsection{Network structure}

To investigate the effect of network structure on fracture we should first learn how to describe the structure of a network such as the collagen network shown in Figure 2.1(a). We can characterize the structure of any network, regardless of its molecular structure, by converting it into a graph with elements or edges, representing the building blocks, and nodes, representing the crosslinks or branching points, as shown in Figure 2.1(c) ${ }^{8,9}$. Via this framework we can systematically study the influence of network structure on elasticity and fracture.

Two features stand out from the graphs of different network materials: i) the highly disordered network structure and ii) the low connectivity (number of connected elements) at all nodes. Almost every soft network material is highly disordered. This can either be through a distribution in the length and thickness 
(a)

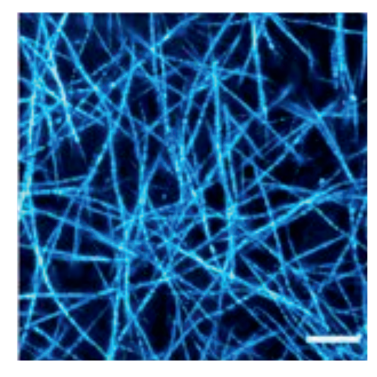

(b)

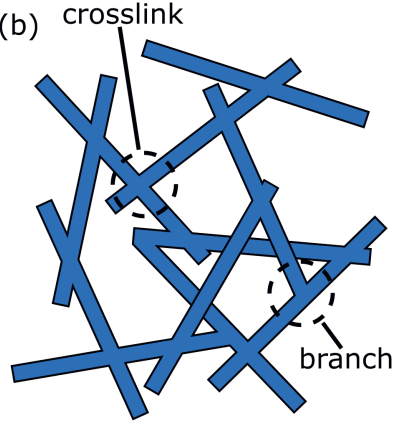

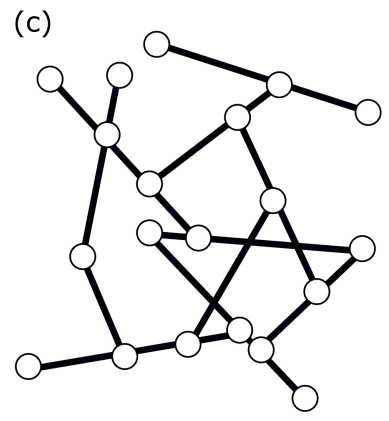

Figure 2.1. Abstraction of the microscopic structure of soft network materials. (a) Microscopy image (confocal reflectance) of a reconstituted bovine type I telocollagen network obtained from Ref. [7]. (b) Abstract representation of a collagen fibril network with crosslinks and branches. (c) Graph representation of the collagen network with nodes and connecting elements (i.e. bonds).

of the elements or through a distribution in the connectivity at the crosslinking sites. The low connectivity at all nodes distinguishes material networks from other networks such as neural networks, social networks and the internet, which typically have several nodes with a very high connectivity.

A disordered network contains many complex structures such as higher order loops and dangling ends. As a result, it is impossible to describe the structure of a network exactly using just a few parameters. This is in contrast to ordered materials, such as crystalline solids, which are built from a repeating unit cell. However, we can approximately describe the structure of the network with a set of three parameters: the average distance between crosslinks $l_{c}$, the average connectivity $\langle z\rangle$ and the density. For the density we either take the line density $\rho$, which is the total length of fibre per volume, the strand density $\phi_{\text {strand, }}$ which is the number of strands per volume sometimes written as $v$, or the crosslink density $\phi_{\text {cross }}$. Note that many fibre networks consist of fibres that are longer than the distance between crosslinks $l_{\text {fibre }}>l_{c}$, i.e. there are multiple crosslinking sites along a fibre. By making assumptions on the process of network formation this allows the description of the network structure with just the values $l_{\text {fibre }}$ and $\rho$, the other parameters will follow from theory ${ }^{4}$.

\subsection{A minimal model for network elasticity and fracture}

To systematically study the effect of these structural parameters on the fracture behaviour of networks, we use a minimal model that can simulate the fracture response of the graphs introduced in the previous paragraph. This model is 
inspired by studies described in literature on the relation between non-linear elasticity and network structure ${ }^{5,10}$.

To construct the model, a network structure is generated from a two-dimensional triangular lattice of size $L$ with a maximum connectivity $z_{\max }=6$. Disorder is introduced by randomly removing bonds with probability $1-p$. After setting the element length $l_{c}$ to unity, $p$ controls both the average connectivity $\langle z\rangle=z_{\max } p$ and the line density $\rho=2 \sqrt{3} * p$. Starting with a lattice seems counter-intuitive, considering that we have been discussing the importance of disorder previously. However, it will become clear later on that the random dilution of bonds introduces enough disorder at the connectivity level to give a good description of real disordered networks.

For simplicity, we describe the elastic response of the elements using a linearly elastic spring with stretching constant $\mu$ and rest length $l_{0}=l_{c}$, such that the force upon extension $F=\mu\left(l-l_{0}\right) / l_{0}$. In Chapter 4 we will also consider bending interactions, but for now we assume that the nodes are freely hinged, i.e. there is no energy penalty for changing the angles between the bonds that connect to the node. We obtain the elastic response of these networks by deforming them under athermal and quasistatic conditions. Under these particular conditions we can neglect thermal fluctuations and other dynamical processes. It also means that the network will always travel through equilibrium states, meaning that we can find the network configuration by minimizing the energy of the system (we use the FIRE algorithm ${ }^{11}$ ).

\subsubsection{Elastic response: Two classes of rigidity}

This approach gives us the tools to look deeper into the origin of network elasticity, and thus how stress is dealt with in a network before bonds start to break. In all cases the elastic response is governed by structural rigidity. Here we can distinguish two cases of rigidity: first-order rigidity and second-order rigidity ${ }^{13}$.

\section{First-order rigidity}

In the linear elastic regime, i.e. at small deformations, the stress-strain response of these networks is highly dependent on the average connectivity. The reason is the relation between connectivity and network rigidity as described by Maxwell ${ }^{14}$. This type of rigidity is also called first-order rigidity and is purely based on topology. A network is first-order rigid if the number of degrees of freedom in the network is at least balanced by the number of constraints (Figure 2.2(a)). In our networks the nodes can move in the $x$ - and $y$-direction, so that the number of degrees of freedom $N_{\text {d.o.f. }}=d N_{\text {nodes }}$ with $d=2$ the dimensionality of the system. 
(a)
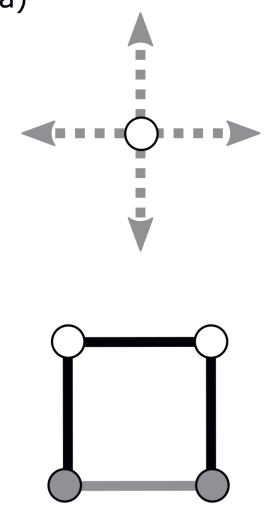
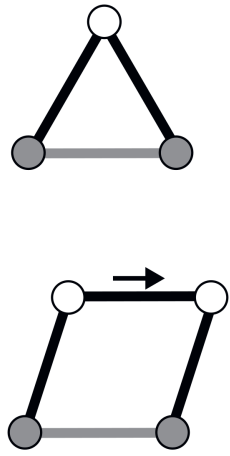

(b)
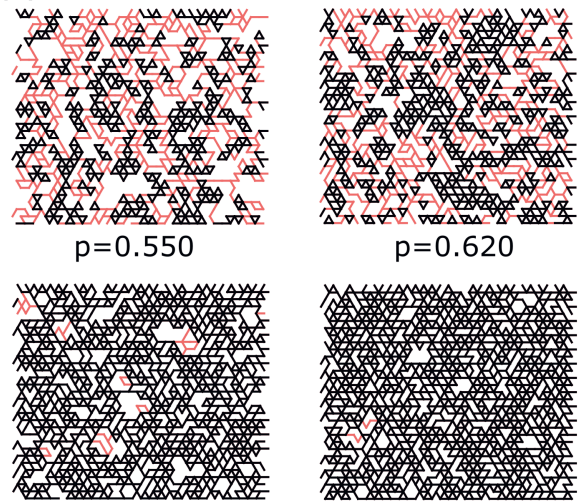

$p=0.680$

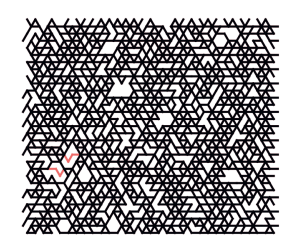

$\mathrm{p}=0.750$

Figure 2.2. Maxwell rigidity is determined by network topology. (a) In two dimensions a node has two degrees of freedom, because it can move along the $x$-axis and along the $y$-axis. A network is rigid according to the Maxwell criterion if the degrees of freedom are matched by at least the same number of constraints. For example, in the triangle shown here the white node can move freely and the grey nodes are fixed. In this system the number of degrees of freedom, matches the number of constraints (black bonds), and thus the triangle is rigid. On the other hand, in the square there are two nodes with four degrees of freedom and three bonds (three constraints the two bottom nodes are fixed, therefore, the grey bond does not count as a constraint). Now the constraints do not match the degrees of freedom and thus the network is not rigid: the upper part can move freely without stretching any of the bonds. (b) Identification of rigid clusters in diluted triangular networks. Rigid cluster (black lines) identified with a pebble game algorithm ${ }^{12}$. The red lines belong to non-rigid parts of the network. For a diluted triangular lattice, a percolating rigid cluster is obtained for $p=2 / 3$. Note that the rigid clusters identified with the pebble game are not unique, slightly different clusters could be identified depending on the exact implementation.

In an undiluted network there are 6 bonds connected to each node and each bond is connected to two nodes such that the number of constraints $N_{\text {constraints }}=3 p N_{\text {nodes }}$. For two-dimensional triangular networks in the thermodynamic limit $(L \rightarrow \infty)$ that means that $p$ should be bigger than $2 / 3$, this value is also called the isostatic point $p_{c}$ (Figure 2.2(b)). Also for smaller networks with periodic boundary conditions $p_{c} \approx 2 / 3$. Rigid networks, those above the isostatic point, have a finite stiffness, implying that a tiny deformation of the network always costs energy, i.e. elements have to be deformed. For these hyperstatic networks $\left(p>p_{c}\right)$ the linear stiffness or modulus (second order-derivative of the energy with respect to the strain) follows a critical scaling with respect to the isostatic point ${ }^{15,16}$. In contrast, a tiny deformation of a subisostatic network $\left(p<p_{c}\right)$ does not cost any energy, i.e. none of the elements is stretched. To explain this difference, we identify rigid 
clusters within networks around $p_{c}$ using a procedure called the pebble game (Figure 2.2(b)). At high $p$, e.g. at $p=0.750$, there is one system spanning cluster, thus deformation of the network requires deformation of this rigid cluster, which requires elements to deform. In contrast, the low $p$ networks contain many small clusters (black) connected by under-constrained nodes/bonds (red). Because these nodes are not completely constrained, the network can rearrange in such a way that the global deformation does not cause any of the bonds to stretch. These rearrangements are called non-affine rearrangements because the displacements of these nodes deviate from the global or affine deformation. Just like the linear modulus, we find a critical scaling in the size of these non-affine deformations approaching the isostatic point from above and from below ${ }^{10,15}$. In summary, a network requires a system spanning cluster in order to be rigid, this is also why it is sometimes referred to as rigidity percolation. This term should not be confused with the term geometrical percolation, which indicates that the top and bottom of the network are connected to each other, for triangular networks this occurs at $p_{g}=0.347$, which is significantly lower than $p_{c}{ }^{17}$.

\section{Second-order rigidity}

That networks with $p<p_{c}$ are not rigid in the linear regime does not mean that networks with a lower connectivity parameter can never become rigid. These networks can be rigidified (provided $p>p_{g}$ ) by applying an external deformation, which aligns the bonds (Figure $2.3(\mathrm{a}))^{19}$. This strain induced rigidity transition can not be predicted based on Maxwell's rigidity criterion, which is purely topological and, therefore, insensitive to external deformation. The rigidity transition is driven by a different class of rigidity which is geometrical in nature, also called second-order rigidity ${ }^{13,20}$. A simple way of understanding this geometrical rigidity transition is to imagine pulling on a slacked (guitar) string. If we represent the string as a chain of elements (Figure 2.3), we find that at low extension the end points can be moved apart without deforming any of the elements in the string. Only when the extension is exactly equal to the total length of the string, the string is pulled taut. This is when the geometrical rigidity transition occurs. The same principle holds for diluted networks as illustrated in Figure 2.3. We also note that this principle is not only relevant for uniaxial extension, but also for biaxial expansion or shear. The non-linear stiffness, the derivative of the stress-strain curve, and the size of the non-affine deformations again show a critical scaling, but now with respect to the strain at which the network becomes rigid; therefore, this strain is called the critical strain $\epsilon_{c}$ for extension or $\gamma_{c}$ for shear ${ }^{5,18}$. This critical strain is a function of $\left|p-p_{c}\right|$ (Figure 2.3(b)). 
(a)

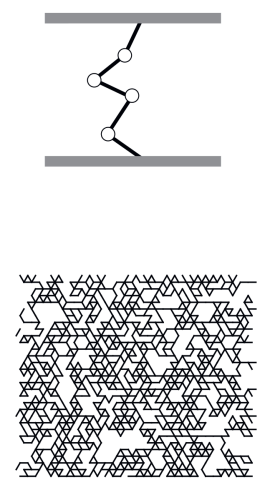

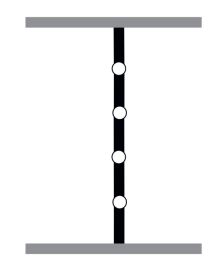

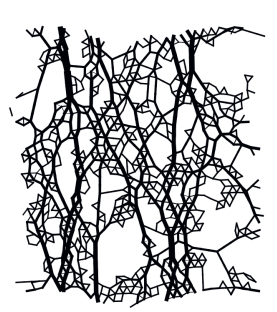

(b)

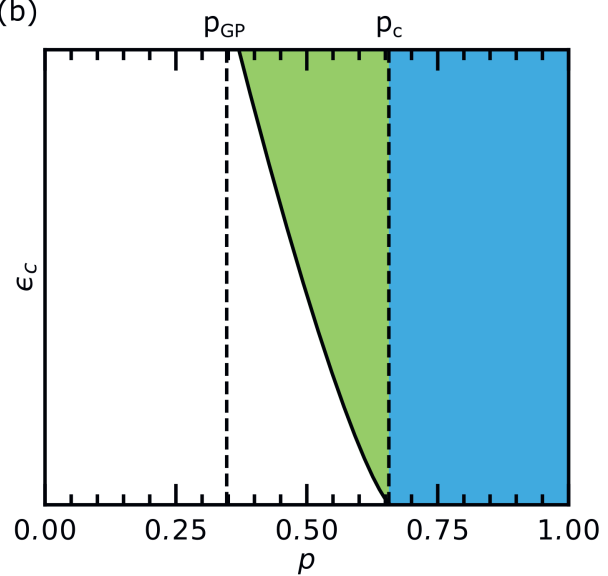

Figure 2.3. Geometrical rigidity is determined by network geometry. (a) Structures that are not first-order rigid, like this chain of elements, can be rigidified under tension. When the elements in the chain are fully aligned, none of the nodes can move without stretching a bond. Similarly, a subisostatic network (bottom left) can be rigidified under tension (bottom right). (b) At what strain $\epsilon$ a network is rigid depends on the connectivity (or in this case the connectivity parameter $p$ ). At and above $p_{\mathcal{C}}$ the network is first-order rigid, thus $\epsilon_{\mathcal{C}}=0.00$ (blue region). Below $p_{\mathcal{c}}$ the critical strain $\epsilon_{c}$ grows with the distance from the isostatic point $\left|p-p_{c}\right|$ (green region). Initially this growth follows a power law ${ }^{15}$ and depends on the deformation mode $\epsilon_{\mathcal{C}} \sim\left|p-p_{c}\right|^{1.3}$ for extension ${ }^{6}$ and $\gamma_{c} \sim\left|p-p_{c}\right|^{1.0}$ for shear ${ }^{18}$, but the scaling deviates when $p$ approaches the point of geometrical percolation $p_{g}{ }^{17,18}$. Below this point geometrical percolation can not be achieved, i.e. the top and bottom of the network are no longer connected; therefore, these networks are never rigid.

\section{Stabilizing fields}

For a three-dimensional network to be first-order rigid, the average connectivity should be at least 6 . However, for most networks it is lower than 4 , thus most soft network materials are subisostatic. Therefore, geometrical rigidity is the most relevant form of rigidity for most soft network materials. This does not mean that soft network materials can be deformed without any effort in the linear regime. Although these networks are not rigid in terms of stretching, they are stabilized by other types of interactions, such as bending interactions or thermal fluctuations, also called stabilizing fields ${ }^{21}$. We already discussed that without a stabilizing field, the network rearranges in a non-affine way to avoid stretching of bonds. This causes changes in the angles between neighbouring bonds. Now, if we include bending interactions, these interactions will resist the non-affine rearrangements, causing an increase in the energy of the system, resulting in a finite stiffness below $\epsilon_{c}$. The scaling of the modulus is now still critical with respect to the critical 

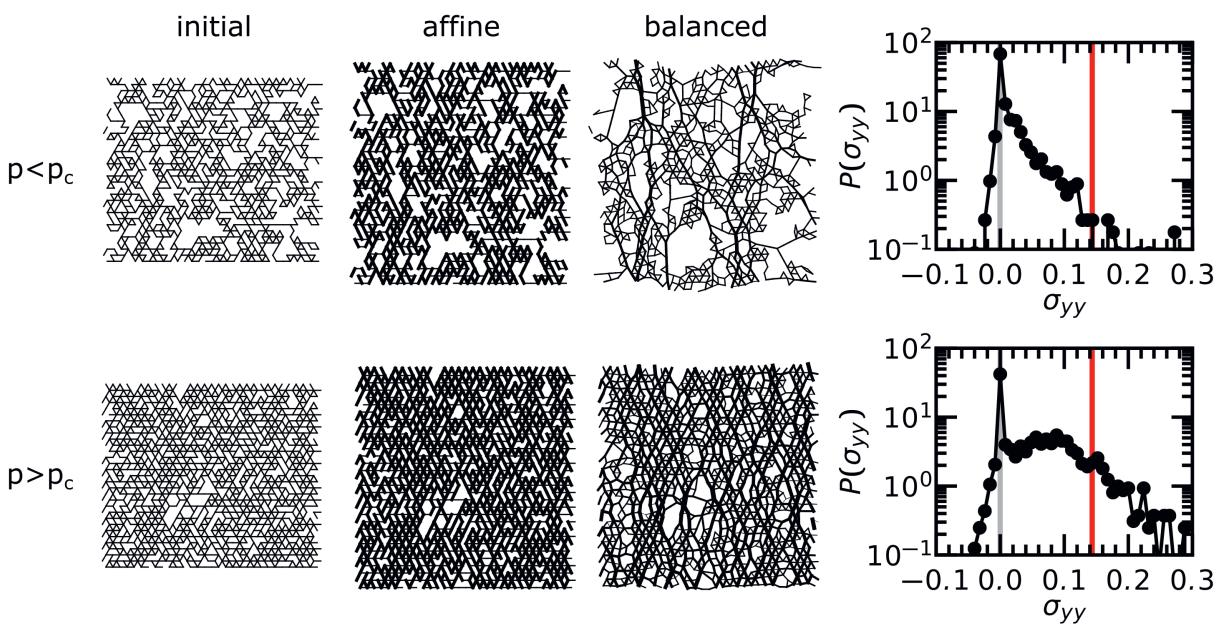

Figure 2.4. Balancing forces leads to an inhomogeneous stress distribution. Affine deformation of an initial network structure $(\Delta \epsilon=0.20)$ leads to an imbalance in the forces at the nodes. After balancing the forces through an energy minimization procedure, we find a highly inhomogeneous distribution in stress both below $p_{c}$ (top row, $p=0.55$ and $L=30$ ) and above $p_{c}$ (bottom row, $p=0.75$ and $L=30$ ). Here we show the distribution in the contribution of each bond to the global stress along the axis of extension. The vertical lines indicate the stress on a diagonal bond (red) and a horizontal bond (grey) under affine deformation. The horizontal bonds are not stretched, because their orientation is perpendicular to the applied strain.

strain; however, the stabilizing field does alter the transition ${ }^{5}$. To what extent the transition is altered depends on the stiffness of the stabilizing field with respect to the stiffness of the elements (see Chapter 3, Chapter 4 and the general discussion for more details). For now we assume that the stabilizing field is weak compared to the stretching interactions, which implies that the stabilizing field can be neglected when looking at the fracture response, because this happens in the stretching dominated regime.

\section{Beyond the rigidity transition}

Predicting when a network becomes structurally rigid is already a difficult problem, but the problem can be well-defined in mathematical terms for a network of inextensible elements (in its most general form this problem has been proven to be NP-hard ${ }^{22}$ ). What governs the behaviour above these rigidity transitions is less well explored, because now the shape of the stretching potential starts to play a role as well. 
We already mentioned that before and after the strain-induced rigidity transition the local deformation is highly non-affine. Extension beyond $\epsilon_{c}$ requires the stretching of bonds. Because of the large non-affine rearrangements the resulting stress distribution is highly inhomogeneous (Figure 2.4). This is in contrast to the expected stress distribution for networks under affine deformation (a common assumption in many constitutive theories) where the stress distribution is homogeneous, especially for our networks. One way to understand this emerging inhomogeneity in stress is that in purely affine deformation the forces are not balanced at the nodes. After balancing the forces by minimizing the energy of the system, we find that large rearrangements have occurred to redistribute the load, leading to a larger heterogeneity in stress. Furthermore, the stress seems to concentrate along paths within the network, that become more apparent at higher strains. These paths are called force chains (or force networks), reminiscent of the force chains observed in granular packings ${ }^{23}$; however, it should be noted that the nature of the forces is quite different (compression versus extension). In terms of stress distributions we see that the distribution is exponential below $p_{c}{ }^{1}$. We note that even though networks with a connectivity $p>p_{c}$ are first-order rigid, also in these networks the stress distribution is heterogeneous. However, now we observe that a bimodal distribution starts to appear, related to the bonds oriented perpendicular and parallel to the applied strain. In this case it seems more like the actual distribution is the affine stress distribution that is perturbed by disorder. This difference with respect to subisostatic networks suggests that the nature of rigidity influences the stress distribution within a network and, therefore, potentially also network fracture.

\subsubsection{Introducing bond rupture}

Considering the large heterogeneities in stress that emerge in disordered networks beyond the rigidity transition, it seems likely that rigidity should also play a role in the fracture response of these networks. This is also suggested by earlier studies ${ }^{6,24}$. To study fracture in the central-force elastic model, we allow breaking of bonds that have exceeded their rest length by a factor $\lambda=\left(l_{\text {break }}-l_{0}\right) / l_{0}$. After every global deformation step we list the bonds that exceed their deformation threshold and remove the most extended bond after which the network is re-equilibrated. This process is repeated until no bond exceeds their deformation threshold. To stay as close as possible to the condition of quasistatic deformation, we adapt the step-size so that (in most cases) only a single bond exceeds its rupture threshold after the deformation step. 


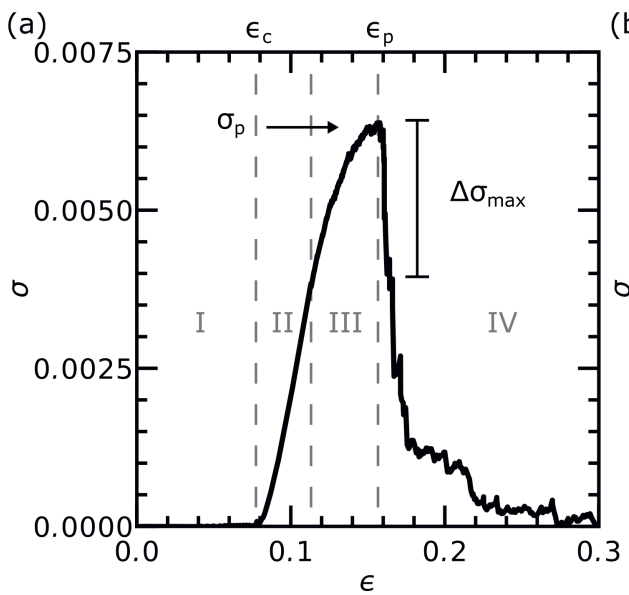

(b)

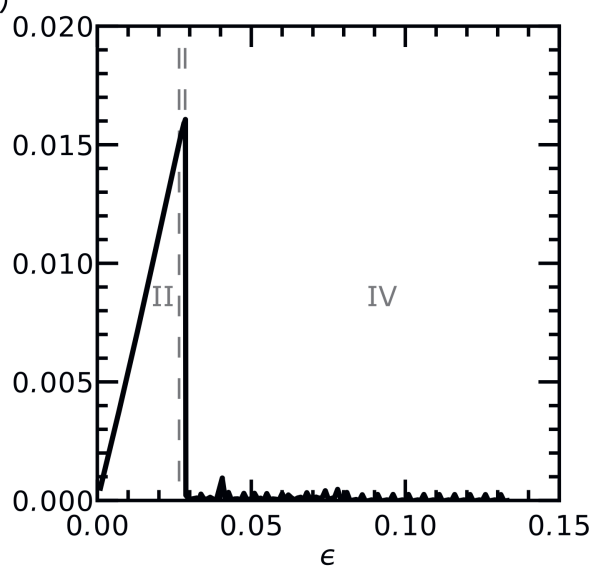

Figure 2.5. Stress-strain response of diluted triangular networks with rupture threshold $\lambda=0.05$ upon uniaxial extension under athermal and quasistatic conditions (a) network below the isostatic point $(L=512, p=0.56, \lambda=0.05)$ and (b) network above the isostatic point $(L=512, p=0.80$, $\lambda=0.05)$.

\section{Mechanical response}

With this model in hand we can investigate the stress-strain response during fracture. In Figure 2.5(a) we show the stress-strain response of a subisostatic network $\left(p<p_{c}\right)$ with a rupture threshold $\lambda=0.05$. The response starts off in the non-rigid regime (Regime I), where the stress is zero because of the absence of stabilizing fields. Followed by a non-linear elastic response starting at $\epsilon_{c}$, during which the force chains develop (regime II). In regime III, bonds start to break, resulting in softening of the stress-strain response. Nevertheless, the carried stress keeps increasing up to a maximum stress $\sigma_{p}$, which indicates that the damaged network can still carry a considerable amount of stress. It is only in the final regime that the stress drops significantly (regime IV), often due to the propagation of a crack. The network is considered broken when percolation from the top to the bottom is lost.

The stress-strain response of a network above $p_{c}$ differs significantly from the stress-strain response of a subisostatic network as shown in Figure 2.5(b). Because these networks are first-order rigid, the stress response immediately starts in the elastic regime (regime II). Furthermore, the strain-softening regime (regime III) is very small, suggesting that damage accumulation prior to the peak stress is negligible. Finally, the response in the final regime (regime IV) is significantly more brittle if we compare it to the response of an equally sized subisostatic network. 
(a)

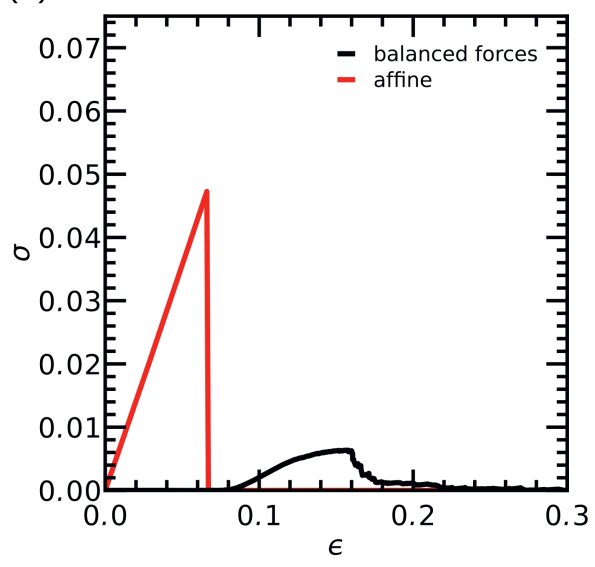

(b)

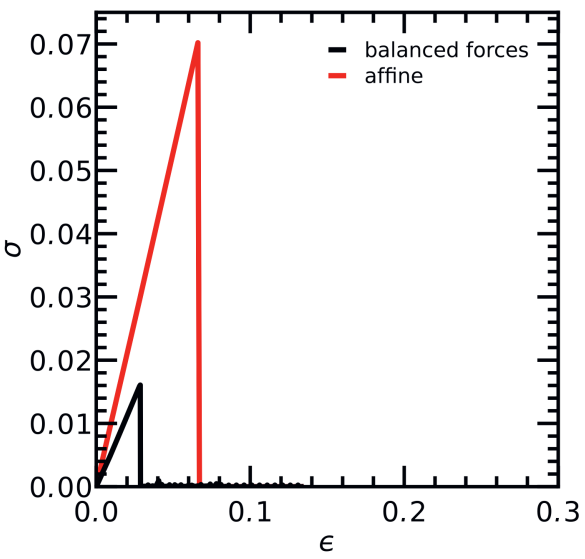

Figure 2.6. Comparison of the response of the model (black) with a prediction for the stress-strain response assuming affine deformation (red). For networks (a) $p<p_{c}(L=512, p=0.56$, $\lambda=0.05)$ and (b) $p>p_{c}(L=512, p=0.80, \lambda=0.05)$.

\section{The role of inhomogeneous stress}

For both networks the inhomogeneous stress distribution resulting from the balancing of forces has a significant impact on the stress-strain response. We illustrate this by comparing the response of the subisostatic network with an affine prediction for the stress in Figure 2.6(a). We clearly see that the actual network response is more stretchable and less strong than the affine prediction. The first distinguishing feature is the absence of the first regime in the affine prediction, which can be ascribed to geometric rigidity as discussed above. Subsequently, we find that in the elastic regime the stiffness (slope of the stress-strain curve) never reaches the stiffness from the affine prediction. This is because of the inhomogeneous distribution in stress. Also, we find significant strain-stiffening in the simulation, which the affine prediction does not show, besides a negligible strain stiffening effect due to the alignment of bonds with the direction of applied strain. Entering the fracture regime, we again see a very different response. While the strain-softening regime is significant in our simulations, this regime is absent in the affine prediction. Because in our model all elements have the same length and all diagonal springs make the same angle with respect to the axis of deformation, all bonds break at the exact same moment in the affine prediction. Furthermore, we see a much more gradual decay in the stress after the peak stress in our simulations.

For a network above $p_{c}$ the simulation response matches qualitatively with the affine prediction, but both the peak stress $\sigma_{p}$ and peak strain $\epsilon_{p}$ are significantly 

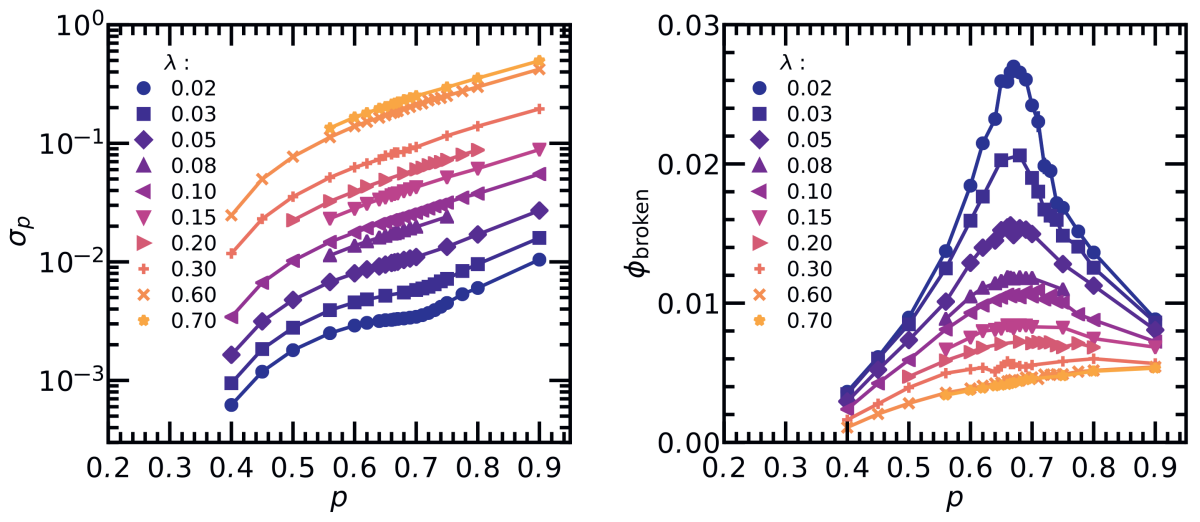

Figure 2.7. Sensitivity to network structure (connectivity parameter) and threshold. (a) Peak stress or strength $\sigma_{p}$ as a function of $p$. (b) Fraction of broken bonds $\phi_{\text {broken }}$ with respect to initial amount of bonds in the diluted network as a function of $p$. For all networks $L=128$.

lower. The lower slope (i.e. stiffness) suggests that small reorganizations are allowed within the networks prior to damage accumulation. The lower $\sigma_{p}$ indicates that stress concentration triggers early fracture. From this comparison it is clear that rigidity qualitatively alters the stress-strain response of subisostatic networks, which we attribute to major non-affine deformations in the network upon deformation and bond rupture. Rigidity does have an effect on the stressstrain response of hyperstatic networks as well, but at least for this example it looks like a (small) perturbation with respect to the affine response.

\section{A rigidity controlled fracture response}

By performing simulations for a range of $p$ and $\lambda$ we can investigate the role of rigidity in a more systematic way ${ }^{25}$. In Figure 2.7(a) we show the dependence of the peak strength on $p$ and $\lambda$. By first approximation, we expect that more bonds and a higher element strength leads to a stronger network. In particular for the affine prediction in the thermodynamic limit we expect that the network breaks catastrophically if one of the diagonal elements is stretched a factor $\lambda$ with respect to its rest length such that $\sigma_{p}=p \mu \lambda \sqrt{4-1 /(1+\lambda)^{2}}$, i.e. we expect a linear increase in $\sigma_{p}$ with $p$. In our simulations there is no linear dependence on $p$ for any $\lambda$. In particular, for low $\lambda$ we find a clear non-linearity in the response around $p_{c}$.

If we extract the fraction of broken bonds $\phi_{\text {broken }}$ as shown in Figure 2.7(b) we see a very clear peak in the fraction of broken bonds around the isostatic point, especially for low element strength (or stretchability). This effect can definitely 
not be expected based on affine deformation. Apparently, the force network can optimally share the load around the isostatic point and potentially recruit inactive bonds in the fracture process, leading to a higher fraction of broken bonds. In other words, these simulations show that in the athermal regime rigidity controls the fracture response. This is in agreement with observations in earlier works by Driscoll et al., Zhang et al. and Berthier et al. ${ }^{6,24,26}$.

The results show that indeed the fracture response is very sensitive to rigidity both below and above $p_{c}$. What immediately becomes clear as well is that the rupture threshold $\lambda$ provides enormous control over the fracture response. Also this phenomenon can be understood in terms of rigidity: for higher $\lambda$-values, bonds will start to break at larger distance $\Delta \epsilon$ from $\epsilon_{c}$. The proposed microscopic picture ${ }^{6}$ is that at $0 \%$ strain every network contains a set of potential force chains, folded to different extents, that can be activated if they are stretched out as portrayed in Figure 2.3(a). For low $\lambda$, force chains will break quickly after their activation, i.e. close to their rigidity transition, so that any load is always carried by a small fraction of continuously exchanging force chains. By contrast, at high $\lambda$ many force chains have already been activated when the first bond breaks. As a result, the breaking of bonds will be less dominated by geometrical rigidity at high $\lambda$.

We note that we are currently only discussing the effect of network structure based on a single parameter. We expect that the average connectivity is the most important determinant of the fracture response and indeed we find a qualitatively similar response for other network structures (2D or 3D) ${ }^{25}$. However, the quantitative differences show that other network characteristics, such as topology and dimensionality, also affect the precise fracture response.

\subsubsection{Role of stress concentration in network fracture}

In Figure 2.7 we observed different trends for $\sigma_{p}$ and $\phi_{\text {broken }}$ as a function of $p$ above and below the isostatic point. This observation introduces the question whether the fracture mechanism for subisostatic networks is different from that for hyperstatic networks. In fact, a mechanistic difference has recently been proposed in literature ${ }^{6}$, which is related to the role of stress concentration in the fracture response. It was suggested that above the isostatic point, fracture of networks of sufficient size is dominated by stress concentration around defects in the network, similar to the classical approach to fracture mechanics described by Griffith ${ }^{27}$. In this description a crack will nucleate and propagate from the largest defect once a particular stress is reached, resulting in global failure once the crack (linear in 2D and planar in 3D) spans the entire system perpendicular to the direction of applied strain. In contrast, Ref. [6] suggests that below the isostatic point 

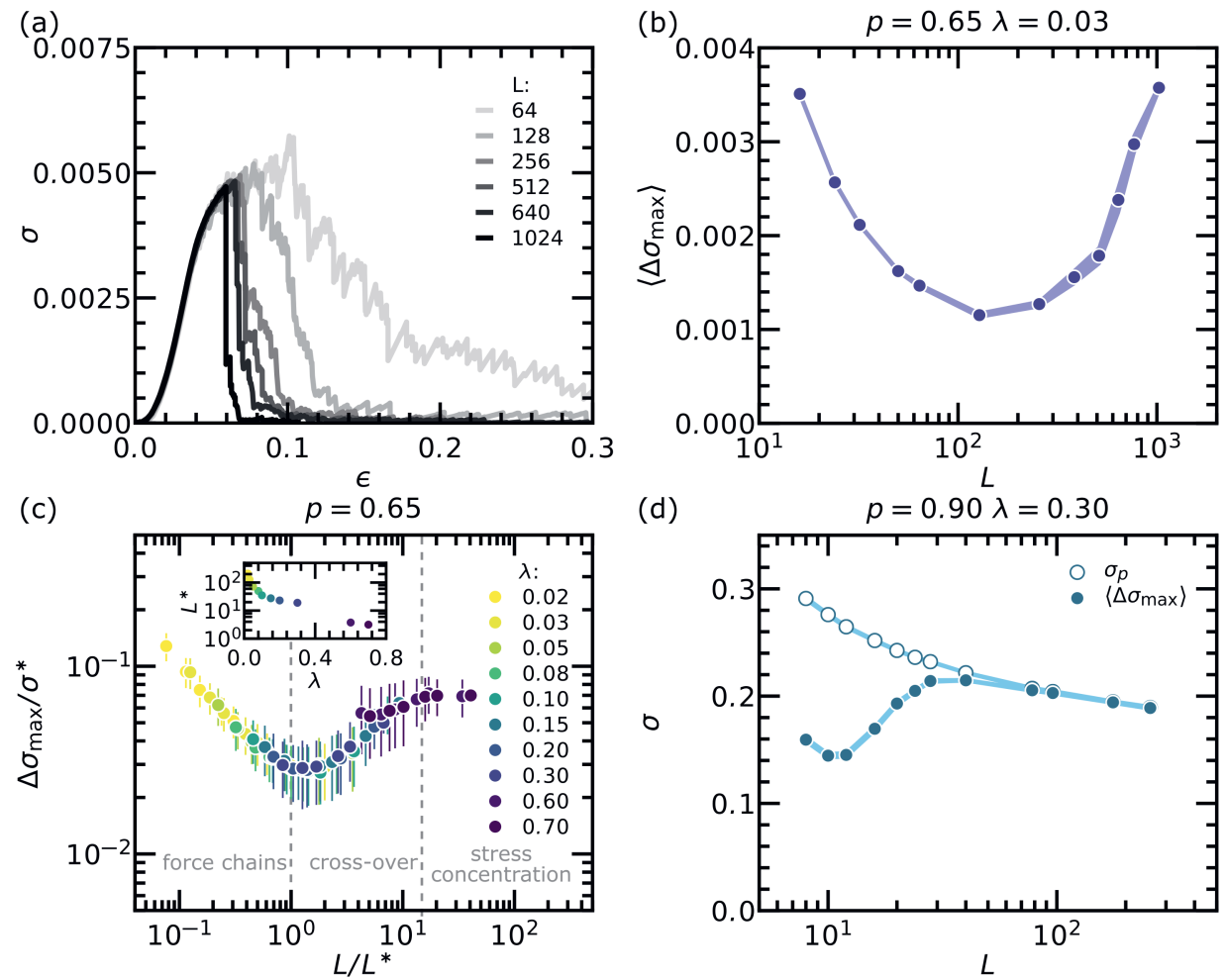

(d)

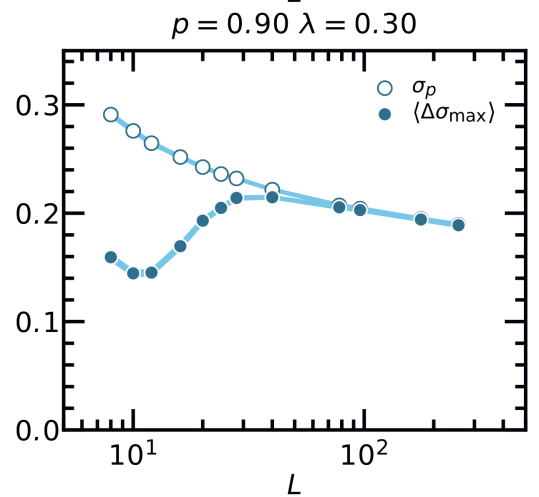

Figure 2.8. Size scaling of the fracture response. (a) Stress-strain responses of subisostatic networks ( $p=0.65$ and $\lambda=0.03$ ) for different networks sizes $L$ (see legend). (b) $\Delta \sigma_{\max }$ for the curves in (a) as a function of system size $L$. The shaded area indicates the standard error of the mean. (c) Master curve obtained by rescaling $\Delta \sigma_{\max }$ curves for $p=0.65$ for different $\lambda$ values (see legend). The characteristic size $L^{*}$ used to rescale the $L$ curves is shown in the inset. The proposed fracture regimes are indicated in the plot. (d) Peak stress $\sigma_{p}$ and $\Delta \sigma_{\max }$ for a hyperstatic network as a function of system size $L(p=0.90$ and $\lambda=0.30)$. The shaded area indicates the standard error of the mean.

stress-concentration does not occur, irrespective of the size of the network and that network failure will not result from a system spanning crack, but from a system-spanning percolating damage cluster, providing an alternative for the standard process of crack nucleation followed by crack propagation. To investigate this hypothesis we look into the size scaling of the fracture response of subisostatic networks in Figure 2.8(a). We find that the global mechanical response of our networks is strongly affected by the size of the network $L$, as will be discussed in detail below. 


\section{Size effects at the peak stress (from diffusive damage to damage nucleation)}

We find that $\sigma_{p}$ and $\epsilon_{p}$ decrease monotonically with network size, according to a power-law decay $\left(\left\langle\sigma_{p}\right\rangle=\alpha L^{-\beta}+\sigma_{p}^{\infty}\right)$ with connectivity-dependent exponents both for subisostatic $(p=0.65)$ and hyperstatic networks $(p=0.70)^{25}$. A decay in the fracture strength has been observed in experiments on strings or wires dating back to Leonardo da Vinci, and has been explained according to the weakest-link argument: the largest defect determines the maximal load and the probability of a large defect increases with system size ${ }^{28,29}$. A decay in the average fracture strength with system size has also been demonstrated for diluted electrical fuse networks, where rigidity does not play a role, and has been shown to depend on the largest defect size ${ }^{30,31}$. However, both the functional form of the decay for wires $^{29}\left(\left\langle\sigma_{p}\right\rangle=-\alpha \ln (L)+\beta\right)$ and for diluted fuse networks ${ }^{30,31}\left(\left\langle\sigma_{p}\right\rangle=\alpha / \sqrt{\ln \left(L^{2}\right)}\right)$ are not found in our simulations, implying that a simple weakest-link argument might not suffice for diluted spring networks. At this moment it is not clear if this deviation results from rigidity effects or long range interactions between different defects in the network, similar to those found in fibre bundle models with a tunable load-sharing range ${ }^{32,33}$.

\section{Size effects after the peak stress (damage propagation)}

For the fracture behaviour after $\sigma_{p}$ the behaviour is more surprising. By eye we can see that the fracture response becomes more abrupt with increasing network size in Figure 2.8(a). We can characterize this abruptness by plotting the maximum drop in stress $\Delta \sigma_{\max }$ versus the system size $L$. Surprisingly, we find a non-monotonic trend in the abruptness (Figure 2.8(b)), in contrast to the monotonic trend in $\sigma_{p}$ and $\epsilon_{p}$. Changing the fracture threshold $\lambda$ has a significant impact on the relation between $\Delta \sigma_{\max }$ and $L$ (see Ref. [25]). However, at a fixed connectivity all curves for different $\lambda$ can be collapsed onto a single master curve as shown in Figure 2.8(c), based on a characteristic length $L^{*}$ and characteristic stress $\sigma^{*}$ that both depend on $\lambda$. This master curve has a distinct shape, that can be found for hyperstatic networks as well. In Figure 2.8(d) $(p=0.90)$ we can even retrieve the whole curve for a single rupture threshold $(\lambda=0.30)$.

All these observations suggest that there is a universal trend in the scaling of the maximum stress drop with network size. At large $L, \Delta \sigma_{\max }$ coincides with $\sigma_{p}$, suggesting that any network, subisostatic or hyperstatic, will fail in an abrupt or brittle way if the network is large enough, in contrast to the hypothesis in Ref. [6]. However, for networks of intermediate size $\Delta \sigma_{\max }$ can be very low, implying a more ductile fracture process. Finally, the maximum stress drop increases again for even smaller networks. 
(a)

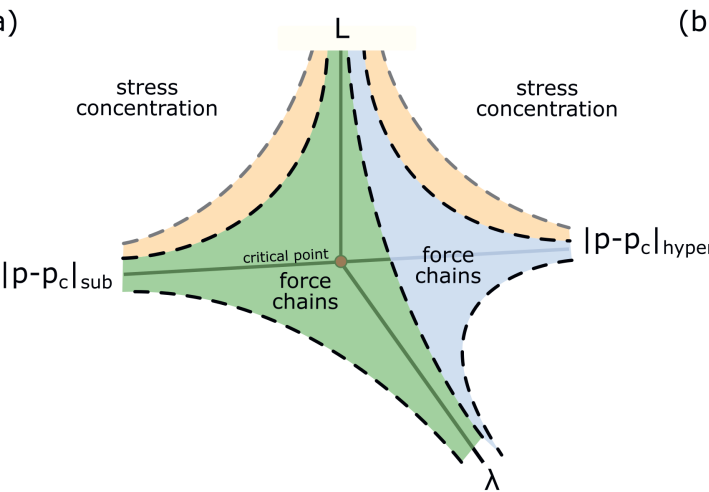

(b)

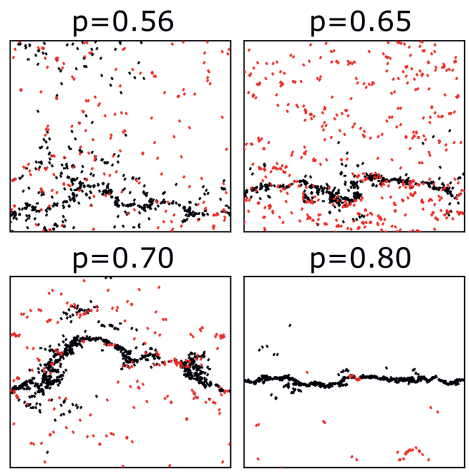

Figure 2.9. Fracture regimes of the minimal central-force elastic model. (a) Fracture regimes as a function of $p, \lambda$ and $L$. (b) Damage patterns for simulations of networks at a range of $p$, $\lambda=0.05$ and $L=256$. Broken bonds are plotted with respect to the initial network configuration. Red bonds represent damage accumulated prior to the peak stress, while black bonds represent damage accumulated at and after the peak stress.

From the scaling we can extract the characteristic size that corresponds to the transition from a brittle to a more ductile fracture response. Here we will define the minimum of the curve as the characteristic size $L^{*}$ (the same value used in the rescaling of Figure 2.8(c)). From the current data it is clear that $L^{*}$ depends on both $p$ and $\lambda$. In particular, $L^{*}$ is significantly larger for subisostatic networks (Figure 2.8(b)) compared to hyperstatic networks (Figure 2.8(d)). A quantitative comparison of $L^{*}$ for different $p$ and $\lambda$ will require additional simulations. However, damage analysis suggests that $L^{*}$ is largest around the isostatic point for low $\lambda$ (not shown $)^{25}$.

\subsubsection{Size-dependent fracture regimes}

The non-monotonic nature of the rescaled abruptness with $L$ and the dependence of $L^{*}$ on $\lambda$ and $p$ suggests that there might be different regimes in the microscopic fracture response of our networks. We hypothesize that at large sizes $\left(L \gg L^{*}\right)$ the fracture response after the peak stress is dominated by stress concentration: stress will concentrate around defects of some sort in the network and macroscopic cracks will emerge from these defects. On the other hand, for small networks $\left(L \leq L^{*}\right)$ we expect a force chain controlled regime after the peak stress, where the networks are so small that upon deformation or bond rupture stress redistribution takes place through the entire network, making concentration of stress around defects difficult. As a result, the post-peak fracture process will be more diffuse and look more 
like damage percolation. Between these regimes we expect a cross-over region as indicated in Figure 2.8(c). The transition from a damage percolation regime to a stress concentration regime is a common observation in the study of statistical fracture mechanics ${ }^{28}$, not only for spring networks ${ }^{24}$, but also for networks of fuses ${ }^{34}$, where rigidity does not play a role, and fibre bundle models ${ }^{33}$, which do not have a network structure. In the general discussion, we will dive deeper into the parallel between our observations and results from literature. The monotonic decrease in $\sigma_{p}$ and $\epsilon_{p}$ suggest that prior to the peak stress this transition does not play a major role. Our interpretation is that prior to the peak stress damage accumulation is dominated by diffuse damage and potentially crack nucleation. In other words, we expect that the propagation of (micro)cracks is negligible before the peak stress.

Combining all these ideas and considering all the possible networks we can create within our model we can now propose a phase diagram for the fracture response in Figure 2.9(a) as a function of $\lambda, L$, and $\left|p-p_{c}\right|$, the distance from the isostatic point. Here the green and the blue surface represent the characteristic parameters where the fracture response changes from brittle to more ductile (or from fracture by stress concentration to fracture by damage percolation) upon approaching the critical point. The orange region marks the cross-over regime. The different fracture regimes should be reflected in different damage patterns. In Figure 2.9(b) we present a few of these damage patterns for networks around the isostatic point at $L=256$ and $\lambda=0.05$. Looking at all the broken bonds, we indeed observe the most spread-out damage pattern around the isostatic point $(p=0.65)$, which seems to be in line with our proposed diagram, that predicts that stress concentration after the peak stress becomes less prominent close to the isostatic point. However, by separating the bonds that break prior to the peak stress (red) from bonds that break at or after the peak stress (black), we find that the majority of diffuse damage around the isostatic point can be attributed to pre-peak damage. For this network size $(L=256)$ the damage pattern after the peak is localized to a meandering crack for all $p$ and the post-peak damage seems to be most diffuse and ductile (damage develops over a wider range of strain) at $p=0.560$, which is not in line with our proposed phase diagram. We note that in these examples diffuse damage is observed both for subisostatic and hyperstatic networks, but that only in subisostatic networks the diffuse damage is spread homogeneously throughout the network. Also for larger subisostatic networks system spanning diffuse damage is observed ${ }^{25}$. A detailed analysis of the microscopic pre-peak and post-peak damage patterns as a function of $p, \lambda$ and $L$ is required to confirm or amend the proposed diagram. 
(a)

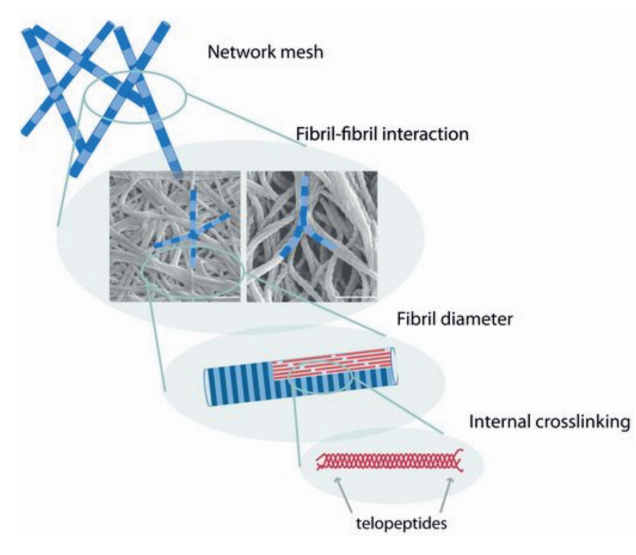

(b)
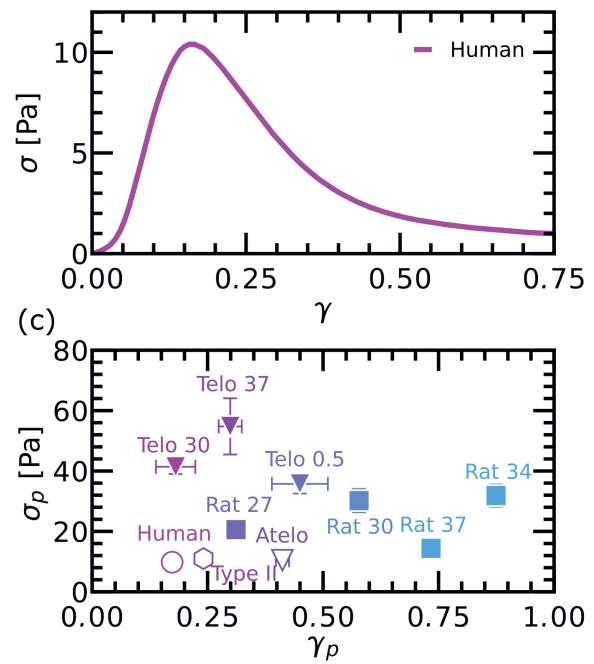

Figure 2.10. Elasticity and fracture of collagen networks. (a) Hierarchical structure of collagen networks. Reprinted from Ref. [7]. (b) Mechanical response of a reconstituted human atelocollagen network. (c) Peak strain $\gamma_{p}$ versus peak stress $\sigma_{p}$ of collagen networks reconstituted from a range of sources and under a range of conditions (see Ref. [7] for details).

\subsection{Putting the theory to the test with experiments: collagen networks}

Collagen networks are the main component of the extracellular matrix of many multicellular organisms, where it provides structure for the tissues and protects them from large mechanical stresses.

Collagen networks are formed from collagen fibrils, which are highly hierarchical structures (Figure 2.10(a)) that self-assemble from tropocollagen strands, itself a macromolecular complex consisting out of three peptides. A network emerges due to the branching and crosslinking of the collagen fibrils. In a biological setting these crosslinks arise from covalent or non-covalent bonds between the fibrils ${ }^{35}$. Using appropriate conditions, the collagen can be extracted from tissues in such a way that the network can be reconstituted under lab conditions, making it possible to perform systematic experiments, such as shear rheology (Figure 2.10(b)).

Networks of collagen are a great candidate for testing the effect of network structure on fracture, as the networks are reasonably well-defined, and it is accepted that these networks behave as athermal systems, in line with our minimal model. In fact, extensive experimental and theoretical work has been done to study the 
(a)

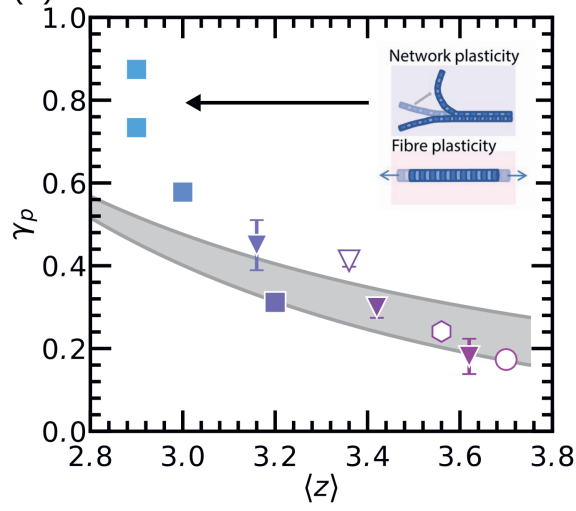

(b)

$\gamma=0.2$

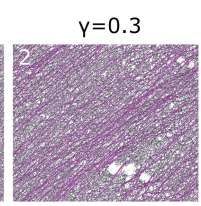

(c)

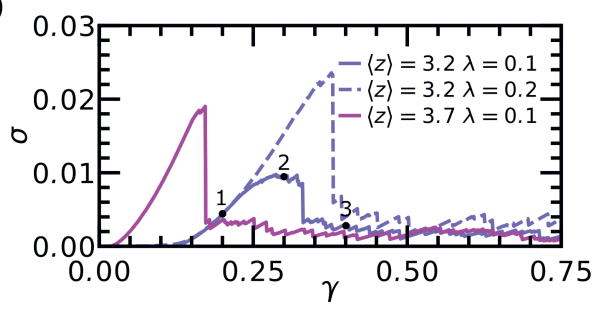

Figure 2.11. The effect of network structure on collagen fracture. (a) Peak strain as a function of connectivity $\langle z\rangle$. The scatter plot represents the experimental response. The grey area indicates the peak strain in simulations on phantom networks with a fracture threshold ranging from $\lambda=0.1$ to $\lambda=0.2$. The inset describes modes of plasticity. (b) Snapshots of a simulation on a phantom network with $\langle z\rangle=3.2$ and $\lambda=0.1$. (c) Stress response of simulations as a function of shear strain $\gamma$, corresponding $\langle z\rangle$ and $\lambda$ are indicated in the legend. The black dots indicate the snapshots shown in (b).

elastic behaviour of these networks, revealing a strong dependence on network structure and rigidity ${ }^{5,10}$. These studies uncovered that the linear elastic response is governed by bending of collagen fibrils, while at higher strains the collagen networks can strain-stiffen over orders of magnitude due to a transition from fibril bending to fibril stretching. The reason for the strain-stiffening is the geometrical rigidity transition discussed in Section 2.3.1 ${ }^{5}$.

Here this exploration is taken one step further by studying the fracture behaviour of a range of collagen networks that vary in either source or formation conditions (Figure 2.10(c)). In particular, we look at the peak strain $\gamma_{p}$, the shear strain at the maximum strength, a value that has proven to be very sensitive to rearrangements at the network level in our minimal central-force elastic model (Figure 2.5(a)). A rule of thumb in material science is that stronger materials are less extensible. Figure 2.10(c) reveals that for collagen networks this trend does not appear. We investigate if we can instead rationalize the fracture response based on structure of the collagen networks in terms of their average network connectivity $\langle z\rangle$.

In Figure 2.11 we plot the peak strain $\gamma_{p}$ as a function of $\langle z\rangle$ for the same collagen networks in Figure 2.10. A clear trend emerges, showing higher peak strains for lower average connectivities. Based on the literature on non-linear elasticity of collagen, such a trend can be expected. The onset of strain-stiffening 
(the transition from bending to stretching) decreases with increasing connectivity ${ }^{36}$. Thus, if we assume that the fibrils only break under tension, we indeed expect that the peak strain decreases with increasing connectivity.

However, we can go one step further in understanding the fracture behaviour of these networks, by comparing the experimental data with simulations on the central-force elastic network model. We use phantom networks ${ }^{7}$, that have been shown to allow a quantitative mapping on experimental results in the elastic regime ${ }^{5,36}$. These phantom networks are again created on a triangular lattice, but now the maximum connectivity $z_{\max }$ in the undiluted network is 4 . This is done by dividing the straight lines, i.e. the fibrils, that cross at every node over two new nodes. The network is brought to the desired $\langle z\rangle$ by randomly removing bonds. Note that we are interested in the fracture response, which only occurs after the rigidity transition, and that we are, therefore, not taking into account bending. Snapshots of these simulations are shown in Figure 2.11(b) with the corresponding stress-strain response in Figure 2.11(c) (blue line). At intermediate strains (regime II in Figure 2.5(c)) we see that force chains emerge, indicated in pink (here the network is still intact). Around the peak stress a crack appears in the network; however, throughout the material we find force chains, which explains why we still measure a significant stress at this strain. Only at higher strains we observe a significant drop in stress, which is related to the expansion of the crack. Now only some force chains are visible around the edges of the crack.

Using these simulations we can investigate the effect of the parameter $\lambda$ on the peak strain. In Figure 2.11(c) we see that a change in $\lambda$ can have a significant impact on $\sigma_{p}$. From experiments on single collagen fibrils, we know that the $\lambda$ values of collagen are roughly between 0.1 and $0.2^{37,38}$. By running simulations within this range for different connectivities, we can make a prediction for $\gamma_{p}$. The expected range for $\gamma_{p}$ is indicated in Figure 2.11(a) with the shaded area. We find that most experimental values lie within or around this area, demonstrating that the phantom model can also be used to map the fracture response and that network structure indeed has a significant influence on the fracture response. However, there are a few outliers which we attribute to plasticity effects either at the network level or at the fibre level. These effects will be discussed in more detail in the general discussion of this thesis.

\subsection{Concluding remarks}

Overall, we can conclude from the studies on a minimal athermal central-force elastic network model that the network structure is mechanically important: it is clear that network rigidity controls the redistribution of load before and during 
the fracture regime. This is apparent from large non-affine rearrangements and the development of force chains, especially around the isostatic point. As a consequence, the strength or stretchability of the element sets the sensitivity to network rigidity. We observe a clear difference in the global mechanical response of subisostatic and hyperstatic networks, as well as some differences in the microscopic (fracture) response.

We find that we can construct a microscopic picture of fracture behaviour for this minimal network model and can even extrapolate towards the thermodynamic limit by varying the size of the network. In particular, this size scaling shows that the mechanical response after the peak stress is highly dependent on network size, with a more ductile response at small $L$ and a brittle response if the network is significantly larger than a characteristic size $L^{*}$. We find that also $L^{*}$ is sensitive to rigidity and is largest at low $\lambda$.

A comparison with an experimental study on collagen reveals that the minimal model can be used to rationalize the fracture behaviour of soft network materials, such as collagen. It should be noted that the mechanical response of collagen is well known to depend on network structure. A comparison with other types of network materials will be essential in order to explore the uses and limits of the framework presented herein. 


\section{List of symbols}

\begin{tabular}{|c|c|}
\hline Symbol & Description \\
\hline$d$ & Dimensionality of the system \\
\hline$F$ & Force \\
\hline$l_{c}$ & Length of the building block (e.g. polymer) between crosslinks \\
\hline$l_{\text {fibre }}$ & Length of a fibre \\
\hline$l$ & Length of an element or spring \\
\hline$l_{0}$ & Rest length of an element or spring \\
\hline$L$ & Lattice size, with $L \times L$ the number of nodes \\
\hline$L^{*}$ & $\begin{array}{l}\text { Characteristic size, corresponding to the cross-over in the size- } \\
\text { sensitivity of } \Delta \sigma_{\max }\end{array}$ \\
\hline$N_{\text {d.o.f. }}$ & Number of degrees of freedom in the system \\
\hline$N_{\text {nodes }}$ & Number of nodes in the system \\
\hline$N_{\text {constraints }}$ & Number of constraints in the system \\
\hline$p$ & $\begin{array}{l}\text { Connectivity parameter, representing the fraction of bonds re- } \\
\text { maining after dilution }\end{array}$ \\
\hline$p_{c}$ & Isostatic point defined in terms of $p$ \\
\hline$p_{g}$ & Geometric percolation point defined in terms of $p$ \\
\hline$z$ & Connectivity, number of bonds connected to a node \\
\hline$z_{\max }$ & Maximum connectivity \\
\hline$\langle z\rangle$ & Average connectivity over all nodes \\
\hline$\gamma$ & Shear strain \\
\hline$\gamma_{c}$ & Critical shear strain \\
\hline$\gamma_{p}$ & Peak strain, strain corresponding to $\sigma_{p}$ \\
\hline$\Delta \sigma_{\max }$ & Abruptness or maximum drop in stress after $\sigma_{p}$ \\
\hline$\epsilon$ & Uniaxial strain \\
\hline$\epsilon_{c}$ & Critical extensional strain \\
\hline$\epsilon_{p}$ & Peak strain, strain corresponding to $\sigma_{p}$ \\
\hline$\lambda$ & Breaking threshold of a spring defined as $\lambda=\left(l_{\text {break }}-l_{0}\right) / l_{0}$ \\
\hline$\mu$ & Stretching constant, related to the spring constant as $k=\mu / l_{0}$ \\
\hline$v$ & Number of (polymer) strands per volume \\
\hline$\rho$ & Line density, total length of fibre per unit volume \\
\hline$\sigma$ & Stress \\
\hline$\sigma_{i j}$ & Component of the virial stress tensor, e.g. $\sigma_{y y}$ \\
\hline
\end{tabular}




$\begin{array}{ll}\sigma_{p} & \begin{array}{l}\text { Peak stress or strength, maximum stress that can be carried by the } \\ \text { network }\end{array} \\ \phi_{\text {cross }} & \text { Number of crosslinks per volume } \\ \phi_{\text {strand }} & \begin{array}{l}\text { Number of (polymer) strands per volume } \\ \phi_{\text {broken }}\end{array} \\ & \begin{array}{l}\text { Fraction of broken bonds relative to the initial amount of bonds in } \\ \text { the diluted network }\end{array}\end{array}$

\section{References}

[1] C. Heussinger, E. Frey, Force distributions and force chains in random stiff fiber networks. European Physical Journal E 24, 47-53 (2007).

[2] J. L. Shivers, J. Feng, A. Sharma, F. C. Mackintosh, Normal stress anisotropy and marginal stability in athermal elastic networks. Soft Matter 15, 1666-1675 (2019).

[3] R. C. Arevalo, P. Kumar, J. S. Urbach, D. L. Blair, Stress heterogeneities in sheared type-I collagen networks revealed by boundary stress microscopy. PLOS ONE 10, 0118021 (2015).

[4] C. P. Broedersz, F. C. Mackintosh, Modeling semiflexible polymer networks. Reviews of Modern Physics 86, 995-1036 (2014).

[5] A. Sharma, A. J. Licup, K. A. Jansen, R. Rens, M. Sheinman, G. H. Koenderink, F. C. Mackintosh, Strain-controlled criticality governs the nonlinear mechanics of fibre networks. Nature Physics 12, 584-587 (2016).

[6] L. Zhang, D. Z. Rocklin, L. M. Sander, X. Mao, Fiber networks below the isostatic point: Fracture without stress concentration. Physical Review Materials 1, 052602 (2017).

[7] F. Burla, S. Dussi, C. Martinez-Torres, J. Tauber, J. van der Gucht, G. H. Koenderink, Connectivity and plasticity determine collagen network fracture. Proceedings of the National Academy of Sciences of the United States of America 117, 8326-8334 (2020).

[8] H. Galina, M. M. Sysło, Some applications of graph theory to the study of polymer configuration. Discrete Applied Mathematics 19, 167-176 (1988).

[9] L. Papadopoulos, M. A. Porter, K. E. Daniels, D. S. Bassett, Network analysis of particles and grains. Journal of Complex Networks 6, 485-565 (2018).

[10] C. P. Broedersz, X. Mao, T. C. Lubensky, F. C. Mackintosh, Criticality and isostaticity in fibre networks. Nature Physics 7, 983-988 (2011).

[11] E. Bitzek, P. Koskinen, F. Gähler, M. Moseler, P. Gumbsch, Structural relaxation made simple. Physical Review Letters 97, 170201 (2006).

[12] D. J. Jacobs, M. F. Thorpe, Generic rigidity percolation: The pebble game. Physical Review Letters 75, 4051-4054 (1995).

[13] O. K. Damavandi, V. F. Hagh, C. D. Santangelo, M. L. Manning, Energetic Rigidity: a Unifying Theory of Mechanical Stability. Preprint at http://arxiv.org/abs/2102.11310 (2021). 
[14] J. C. Maxwell, On the calculation of the equilibrium and stiffness of frames. The London, Edinburgh, and Dublin Philosophical Magazine and Journal of Science 27, 294-299 (1864).

[15] M. Wyart, H. Liang, A. Kabla, L. Mahadevan, Elasticity of floppy and stiff random networks. Physical Review Letters 101, 215501 (2008).

[16] A. Zaccone, E. Scossa-Romano, Approximate analytical description of the nonaffine response of amorphous solids. Physical Review B - Condensed Matter and Materials Physics 83, 184205 (2011).

[17] M. F. Sykes, J. W. Essam, Exact critical percolation probabilities for site and bond problems in two dimensions. Journal of Mathematical Physics 5, 1117-1127 (1964).

[18] S. Arzash, J. L. Shivers, F. C. MacKintosh, Finite size effects in critical fiber networks. Soft Matter 16, 6784-6793 (2020).

[19] M. Merkel, K. Baumgarten, B. P. Tighe, M. L. Manning, A minimal-length approach unifies rigidity in underconstrained materials. Proceedings of the National Academy of Sciences of the United States of America 116, 6560-6568 (2019).

[20] R. Connelly, W. Whiteley, Second-order rigidity and prestress stability for tensegrity frameworks. SIAM Journal on Discrete Mathematics 9, 453-491 (1996).

[21] A. J. Licup, A. Sharma, F. C. Mackintosh, Elastic regimes of subisostatic athermal fiber networks. Physical Review E 93, 41-44 (2016).

[22] T. G. Abbott, Generalizations of Kempe's Universality Theorem, Ph.D. thesis, Massachusetts Institute of Technology (2008).

[23] S. Ostojic, E. Somfai, B. Nienhuis, Scale invariance and universality of force networks in static granular matter. Nature 439, 828-830 (2006).

[24] M. M. Driscoll, B. G. G. Chen, T. H. Beuman, S. Ulrich, S. R. Nagel, V. Vitelli, The role of rigidity in controlling material failure. Proceedings of the National Academy of Sciences of the United States of America 113, 10813-10817 (2016).

[25] S. Dussi, J. Tauber, J. Van Der Gucht, Athermal Fracture of Elastic Networks: How Rigidity Challenges the Unavoidable Size-Induced Brittleness. Physical Review Letters 124, 18002 (2020).

[26] E. Berthier, J. E. Kollmer, S. E. Henkes, K. Liu, J. M. Schwarz, K. E. Daniels, Rigidity percolation control of the brittle-ductile transition in disordered networks. Physical Review Materials 3, 075602 (2019).

[27] A. A. Griffith, The Phenomena of Rupture and Flow in Solids. Philosophical Transactions of the Royal Society A: Mathematical, Physical and Engineering Sciences 221, 163-198 (1921).

[28] M. J. Alava, P. K. V. V. Nukala, S. Zapperi, Statistical models of fracture. Advances in Physics 55, 349-476 (2006).

[29] J. Fontana, P. Palffy-Muhoray, St. Petersburg Paradox and Failure Probability. Physical Review Letters 124, 245501 (2020).

[30] P. M. Duxbury, P. L. Leath, P. D. Beale, Breakdown properties of quenched random systems: The random-fuse network. Physical Review B 36, 367-380 (1987). 
[31] C. Manzato, A. Shekhawat, P. K. V. V. Nukala, M. J. Alava, J. P. Sethna, S. Zapperi, Fracture strength of disordered media: Universality, interactions, and tail asymptotics. Physical Review Letters 108, 065504 (2012).

[32] S. Roy, Predictability and strength of a heterogeneous system: The role of system size and disorder. Physical Review E 96, 042142 (2017).

[33] S. Roy, S. Biswas, P. Ray, Modes of failure in disordered solids. Physical Review E 96, 063003 (2017).

[34] A. Shekhawat, S. Zapperi, J. P. Sethna, From damage percolation to crack nucleation through finite size criticality. Physical Review Letters 110, 185505 (2013).

[35] F. Burla, Y. Mulla, B. E. Vos, A. Aufderhorst-Roberts, G. H. Koenderink, From mechanical resilience to active material properties in biopolymer networks. Nature Reviews Physics 1, 249-263 (2019).

[36] K. A. Jansen, A. J. Licup, A. Sharma, R. Rens, F. C. MacKintosh, G. H. Koenderink, The Role of Network Architecture in Collagen Mechanics. Biophysical Journal 114, 2665-2678 (2018).

[37] M. Guthold, W. Liu, E. A. Sparks, L. M. Jawerth, L. Peng, M. Falvo, R. Superfine, R. R. Hantgan, S. T. Lord, A comparison of the mechanical and structural properties of fibrin fibers with other protein fibers. Cell Biochemistry and Biophysics 49, 165-181 (2007).

[38] S. M. Iqbal, D. Deska-Gauthier, L. Kreplak, Assessing collagen fibrils molecular damage after a single stretch-release cycle. Soft Matter 15, 6237-6246 (2019). 



\title{
Chapter 3
}

\section{The role of temperature in the rigidity-controlled fracture of elastic networks}

\begin{abstract}
We study the influence of thermal fluctuations on the fracture of elastic networks, via simulations of the uniaxial extension of central-force spring networks with varying rigidity. Studying their failure response, both at the macroscopic and microscopic level, we find that an increase in temperature corresponds to a more homogeneous stress distribution and induces thermally activated failure of springs. As a consequence, the material strength decreases upon increasing temperature, the microscopic damage spreads over a larger area and a more ductile fracture process is observed. These effects are modulated by network rigidity and can therefore be tuned via the network connectivity and the rupture threshold of the springs. Knowledge of the interplay between temperature and rigidity improves our understanding of the fracture of elastic network materials, such as (biological) polymer networks, and can help to refine design principles for tough soft materials.
\end{abstract}

Justin Tauber, Aimée R. Kok, Jasper van der Gucht \& Simone Dussi

"The role of temperature in the rigidity-controlled fracture of elastic networks" Soft Matter 16, 9975-9985 (2020) 


\subsection{Introduction}

Many soft materials have a microstructure that consists of a disordered network. The building blocks that constitute this network range from stiff fibres ${ }^{1}$ in paper or textiles, to semiflexible filaments in biological cells and tissues ${ }^{2-4}$, to flexible polymer chains in elastomers ${ }^{5}$. The network architecture is known to be important for the mechanical response and failure of these materials ${ }^{1,2,6,7}$; yet, most theoretical studies describe the failure response via continuum mechanics ${ }^{8}$ or using a meanfield approach ${ }^{9}$, which ignore details of the network topology and neglect the role of structural disorder. Such models thus cannot provide a microscopic understanding of the failure process. Therefore, coarse-grained network models have been developed, which treat the material as a disordered network of elastic springs, and which explicitly take connectivity and disorder into account ${ }^{10-13}$. Recent computational studies, complemented with experiments on architectured elastic networks, have shown that under athermal and quasistatic conditions the elastic response and failure behaviour of an elastic network is controlled by both the connectivity of the network and the strength of the individual elements ${ }^{12-15}$. The network architecture, in particular its connectivity, determines the network rigidity. Commonly, the average connectivity of a random network is described using the average number of bonds per crosslink. Central-force spring networks, where the elements only resist stretching, are rigid above a connectivity $2 d$, with $d$ the dimensionality of the network; this is called the isostatic point ${ }^{16}$. Below the isostatic point, central-force spring networks are mechanically floppy. However, simulations have shown that even floppy or subisostatic networks can be rigidified by an external deformation $13,17,18$ or by the presence of additional interactions such as a bending rigidity $6,10,11,19-23$. These recent studies suggest that if the static network structure and the element strength of a soft athermal material are known, the material response can be predicted based on the physical concept of rigidity.

However, these athermal network models completely ignore thermal fluctuations. While this may be justified for networks composed of very stiff fibres, it is highly questionable for softer networks. For example, the mechanics of flexible polymer networks, such as elastomers and hydrogels, are known to be governed by thermal fluctuations and entropy, while network connectivity is usually not taken into consideration ${ }^{24,25}$. This raises the question of how connectivity and thermal fluctuations interplay for networks consisting of fibres of intermediate stiffness, as found, for example, in many biological materials. Recent Monte Carlo simulations for such networks suggest that in the presence of thermal fluctuations the linear modulus is dependent on both rigidity and temperature. In particular, it is shown that thermal fluctuations can stabilize (subisostatic) central-force spring networks 
at the network level in a similar way to bending interactions ${ }^{26,27}$. Also, when looking at failure, it is predicted that the average external force required to break a single element or bond is typically reduced in presence of thermal fluctuations ${ }^{28,29}$. However, experiments on polymer networks ${ }^{30}$ that demonstrate the presence of this thermally activated failure process in network materials also imply that in networks the thermally activated failure is enhanced. These results suggest that, when thermal fluctuations are present, the elastic and failure response can be controlled by temperature, but also by the structure of the network. However, it remains unclear how these two parameters together govern the failure process.

In this chapter, we explore to what extent network rigidity controls the influence of thermal fluctuations on the failure behaviour of an elastic material. To this end, we study the response of diluted central-force spring networks (Figure 3.1), similar to previous studies ${ }^{12-14,26,27}$. To introduce thermal fluctuations into these systems we perform Langevin dynamics simulations, which means that the networks are effectively embedded in an implicit solvent. We find that the strength of the networks is dependent on temperature and that the effect of the thermal fluctuations is coupled to the rigidity of the network. The simple structure of the model allows us to highlight the interplay between rigidity and temperature, and to provide insight in the underlying microscopic mechanisms of stress homogenization and diffuse failure.
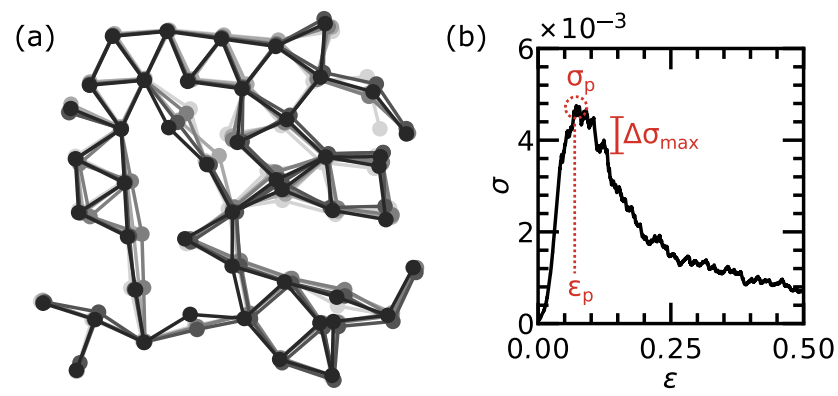

Figure 3.1. Fracture of thermal spring networks. (a) Portion of a spring network under $1.5 \%$ extensional strain. Several snapshots (corresponding to different shades of gray) are overlaid to indicate the effect of thermal motion. (b) Example of the stress-strain response of a diluted spring network ( $p=0.65, \lambda=0.03, T^{*}=10^{-4}, L=128$ ). We highlight the peak stress $\sigma_{p}$, the corresponding peak strain $\varepsilon_{p}$, and the maximum drop in stress $\Delta \sigma_{\text {max }}$. 


\subsection{Model and methods}

We consider diluted spring networks with a 2D triangular topology consisting of $L \times L$ nodes separated by a distance $\ell_{0}$. Nearest neighbours are connected by bonds, which gives a maximum network connectivity $z_{\max }=6$. The network is subsequently randomly diluted by removing a fraction $1-p$ of the bonds, such that the average connectivity becomes $\langle z\rangle=p z_{\max }$. Periodic boundary conditions are employed in all directions. The bonds are harmonic (linear) springs with spring constant $\mu$ and rest length $\ell_{0}$. Excluded volume interactions are not present in the system. During fracture simulations, bonds break irreversibly when their relative extension $\Delta \ell / \ell_{0}$ exceeds a rupture threshold $\lambda$, which is the same for all the springs. We will focus on networks with $\lambda=0.03$.

Simulations are performed using LAMMPS ${ }^{31}$ and nodes follow Langevin dynamics:

$$
m \frac{d^{2} \boldsymbol{r}}{d t^{2}}=\boldsymbol{F}-\zeta \frac{d \boldsymbol{r}}{d t}+\sqrt{2 m \zeta k_{B} T} R(t)
$$

where $\boldsymbol{F}=\mu \Delta \ell, m$ is the mass, $\zeta$ is a friction coefficient, $k_{B}$ the Boltzmann's constant, $T$ the temperature and $R(t)$ white noise with zero-mean. The terms on the right-hand side of Equation 3.1 describe all the contributions to the movement of the nodes: the elastic interaction with the network via the springs (first term), a velocity dependent friction term with the implicit solvent (second term), and a stochastic term which represents random kicks from the molecules in the implicit solvent (third term). The simulation is performed in reduced units with $\ell_{0}=1$ as the unit for distance, $m=1$ the unit for mass, $\mathcal{E}=1$ the unit for energy and $k_{\mathrm{B}}=1$ the Boltzmann constant. By replacing these reduced units with values corresponding to an experimental system, the results of this model can be expressed in terms relevant for that system (see Section 3.A.5 for some examples). The integration time step is set to $\delta t=0.001 \tau$, where $\tau=\sqrt{m \ell_{0}^{2} / \mathcal{E}}$ is the unit time of our simulations. The spring stiffness is set to $\mu=2000 \mathcal{E} / \ell_{0}^{2}$. In our analysis, we will use the reduced temperature $T^{*}=k_{\mathrm{B}} T /\left(\mu \ell_{0}^{2}\right)$, indicating the ratio between thermal and elastic energies. We set the friction with the implicit solvent to $\zeta=10 \varepsilon \tau / \ell_{0}^{2}$. The friction coefficient controls how quickly fluctuations in the system are damped by the implicit solvent. A high friction coefficient, e.g. a high solvent viscosity, results in fast damping of fluctuations, while a low friction coefficient results in slow damping of fluctuations so that inertia plays a dominant role in the system dynamics. The friction coefficient chosen here corresponds to the intermediate (underdamped) regime. In addition, we can define a timescale $\tau_{0}=\zeta / \mu$, the time that a node requires to travel a distance $\ell_{0}$ if it is subjected to a force $\mu \ell_{0}$. For our set of simulations $\tau_{0}=5 \times 10^{-3} \tau$. 


\subsubsection{Measuring linear modulus and non-affinity}

We calculate the linear Young modulus $E$ from the difference in average stress at $0 \%$ strain and $1.5 \%$ strain. At both strain values the system is equilibrated for $100 \tau$ and averaged over $1900 \tau$. The stress $\sigma$ is defined as the $y y$-component (along the deformation axis) of the virial stress tensor normalized by $\mu$. In the following we only show the virial stress based on mechanical interactions. Inclusion of the kinetic component of the virial stress does not change the conclusions of this chapter (Figure 3.A.4). Due to the thermal fluctuations a hydrostatic component is present, even at $0 \%$ strain (Figure 3.A.5). Analysis using the deviatoric stress shows that subtracting the hydrostatic component does not alter our conclusions (Figure 3.A.6). We also calculate the non-affinity parameter defined as

$$
\Gamma_{\text {mech }}=\frac{\left\langle\left(\overline{\boldsymbol{r}}-\overline{\boldsymbol{r}}_{\mathrm{aff}}\right)^{2}\right\rangle}{\varepsilon^{2} \ell_{0}^{2}},
$$

where $\overline{\boldsymbol{r}}$ is the time averaged position of the individual nodes after an applied deformation and $\bar{r}_{\text {aff }}$ the position assuming an affine displacement with respect to the time averaged position of individual nodes at rest. $\varepsilon$ is the strain and $\langle$. indicates the average over all nodes. For these simulations, both non-percolating clusters and primary dangling ends (i.e. nodes that are only connected to one bond after dilution) are iteratively removed from the network.

\subsubsection{Non-linear elasticity and fracture}

The network is uniaxially deformed in the $y$-direction up to $100 \%$ strain with a fixed strain rate of $\dot{\varepsilon}=0.001 \tau^{-1}$ (i.e. $0.0001 \%$ deformation per unit time) while the lateral size is kept constant. We remap the node positions between time steps, temporarily enforcing affine deformation. The deformation is relatively slow compared to the $\tau_{0}, \dot{\varepsilon}=5 \times 10^{-6} \tau_{0}^{-1}$, which indicates the system has time to respond to the affine deformation via structural rearrangements. Results for varying $\dot{\varepsilon}$ are reported in the Appendix (Figure 3.A.3). A quick equilibration run of $50 \tau$ precedes the deformation. The network response is quantified by looking at the stress $\sigma$ as a function of strain $\varepsilon$. In addition, we follow the instantaneous non-affine response

$$
\Gamma_{=} \frac{\left\langle\left(\boldsymbol{r}-\overline{\boldsymbol{r}}_{\mathrm{aff}}\right)^{2}\right\rangle}{\varepsilon^{2} \ell_{0}^{2}},
$$

where the affine response is calculated with respect to the equilibrium position of the nodes at $0.0 \%$ strain (averaged over 1900 $\tau$ ). Please note that $\Gamma$ is only based on 
the instantaneous positions and the non-affine response is therefore a combination of both rigidity controlled non-affine network rearrangements and instantaneous thermal fluctuations (see Appendix for details on the relation between $\Gamma$ and $\left.\Gamma_{\text {mech }}\right)$.

In fracture simulations bonds are broken every 100 steps (i.e. $0.1 \tau)$ when the connected nodes are separated by a distance more than $\ell_{0}+\lambda \ell_{0}$. While the interval chosen for the evaluation of rupture events influences the stress-strain curves somewhat, it does not affect the conclusions of this chapter (Figure 3.A.8 and Figure 3.A.9). From the measured stress-strain curves we extract several quantities (Figure 3.1). All quantities are averaged over several configurations and expressed in reduced units. The peak stress $\sigma_{p}$ is defined as the highest measured stress, and the peak strain $\varepsilon_{p}$ is its corresponding strain value. The maximum stress drop $\Delta \sigma_{\max }$ is calculated according to a procedure ${ }^{13,32}$ where we i) calculate the derivative of the stress-strain curve, ii) make a list of consecutive data points which have a negative derivative and note the initial and final strain of each interval, iii) calculate the stress drops by subtracting the stress at the final strain from the stress at the initial strain, iv) identify the largest stress interval, which corresponds to maximum stress drop $\Delta \sigma_{\max }$. For the stress distribution analysis, we make instantaneous histograms of the bond lengths $\ell_{i}$ during the simulation at every percent strain. Based on these histograms, we calculate the excess kurtosis

$$
\kappa_{e}=\frac{\sum_{i}\left(\ell_{i}-\langle\ell\rangle\right)^{4}}{N_{b} s^{4}}-3,
$$

where $s$ is the standard deviation of the histogram, $N_{b}$ the total number of bonds, and $\langle\ell\rangle$ the average bond length. For all these parameters the standard error is calculated as the standard deviation divided by the number of sampled configurations (standard error of the mean). The errors are shown when they are larger than the symbols displayed in the graphs.

\subsection{Results and discussion}

Using Langevin dynamics simulations, we uniaxially deform diluted triangular central-force spring networks to study both linear and non-linear network mechanics. By analysing the network response on both a macroscopic and microscopic level, we gain insight into the effects of thermal fluctuations on the fracture process of spring networks, as well as how the rigidity controlled failure of networks is affected by thermal fluctuations. Throughout the manuscript we use the reduced temperature $T^{*}=k_{\mathrm{B}} T /\left(\mu \ell_{0}^{2}\right)$ which is a measure for the energy of the thermal fluctuations relative to the energy required to extend the springs in the network. 


\subsubsection{The effect of thermal fluctuations on linear elasticity}

First, we study the effects of thermal fluctuations on the linear elasticity of centralforce spring networks to check if the rigidity-dependent elasticity observed via Monte Carlo simulations ${ }^{26,27}$, an equilibrium method, can also be observed in our dynamical model based on the Langevin equation (Equation 3.1).

In Figure 3.2(a) we plot the linear modulus $E$ of the network as a function of the network connectivity factor $p$ for several reduced temperatures $T^{*}$. The linear modulus $E$ describes the resistance of a network to deformation, and we observe that networks below the isostatic point of mechanical stability ${ }^{16}$ (i.e. networks below $p_{\text {iso }} \approx 0.66$ ) display a finite linear modulus $E$, which would be absent for athermal systems (in the limit of $T=0$ ). This finite $E$ is an effect of entropic stiffness, a temperature-dependent phenomenon. Please note that this entropic stiffness is a
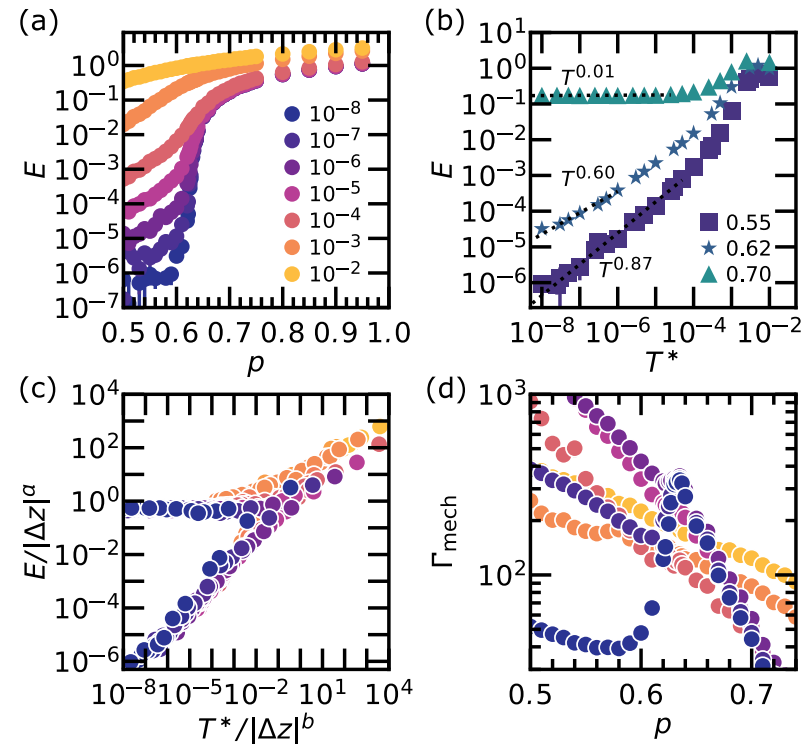

Figure 3.2. Characterization of the linear elastic response for diluted triangular networks of fixed system size $L=128$. (a) Young's modulus $E$ as a function of the connectivity parameter $p$ for different temperatures $T^{*}$. (b) Temperature dependence of $E$ for networks below, around, and above the isostatic point (value of $p$ indicated in the legend). The dashed lines indicate the power-law fit $T^{\alpha}$. (c) Rescaling of Young's modulus according to Ref. [26] with $a=1.4$, $b=2.8$, and $z_{c}=3.78$. (d) The non-affinity parameter $\Gamma_{\text {mech }}$ at $1.5 \%$ strain as a function of $p$ for different temperatures, same legend as (a). Every data point is based on simulations of at least 10 independent configurations. 
network effect and is not the same as the entropic elasticity arising from individual polymer chains. As reported in literature ${ }^{26,27}$, the scaling of the linear modulus with temperature $E \propto T^{\alpha}$ depends on both connectivity and temperature itself. By plotting $E$ as a function of $T^{*}$ we extract the scaling exponent $\alpha$ from a power-law fit for three different values of $p$, as shown in Figure 3.2(b). For a subisostatic network with a connectivity parameter $p=0.55$ the linear modulus scales with $\alpha=0.84$, which roughly corresponds to the dependence found in the anomalous regime as defined in Ref. [26], where a shear deformation was instead considered. It was argued that the disordered network structure causes this sub-linear dependence. Whilst there is a clear dependence of the linear modulus on the temperature below the isostatic point, the curves for the different temperatures start to converge when approaching a structurally rigid network (Figure 3.2(a)). Accordingly, stiff networks display temperature insensitivity $(\alpha \approx 0)$, as can be seen for a network with $p=0.70$ in Figure 3.2(b). As $T^{*}$ increases, however, the network connectivity becomes less important as the energetic contribution arising from the structural rigidity becomes negligible compared to the entropic elasticity. This is noticeable in Figure 3.2(a) where the curve for $T^{*}=10^{-2}$ is roughly flat for the entire $p$-range, and also in Figure 3.2(b) where for $p=0.70, E$ increases for $T^{*}>10^{-3}$. As predicted in Ref. [26], we also find a different scaling for networks close to the isostatic point, see, e.g., the curve for $p=0.62$ in Figure 3.2(b). Although the exponent $\alpha$ is slightly different from the findings of Ref. [26] (where shear deformation and different simulation methods were employed), we were also able to obtain critical rescaling as shown in Figure 3.2(c). We can conclude that there are different regimes of dependence for the linear modulus on the temperature based on both rigidity and temperature.

Furthermore, we find similar rigidity-dependent behaviour of the thermal fluctuations in the non-affinity parameter $\Gamma_{\text {mech }}$ (Equation 3.2), reported in Figure 3.2(d) as a function of $p$ for different $T^{*}$. The non-affinity of the network describes how much the time-averaged local deformation differs from the global (externally imposed) deformation. At low $T^{*}$ we find a peak in non-affine deformation around the isostatic point $(p \approx 0.66)$. This peak arises from the tendency of the spring network to minimize internal stress upon deformation. If the spring network is far below the isostatic point, the stress can be reduced significantly by a small amount of non-affine rearrangements, while at the isostatic point many non-affine rearrangements are required. At the isostatic point, an increase in $T^{*}$ decreases $\Gamma_{\text {mech, }}$ which suggests that thermal fluctuations act as a stabilizing field, similar to the bending rigidity in fibre networks. ${ }^{10}$ However, we note that the effect of thermal fluctuations is always present, even without external deformations, leading to structural rearrangements in the rest state (Figure 3.A.1). Above the 
isostatic point, we observe that the non-affinity converges for most values of $T^{*}$ (see Figure 3.A.1 for details), which indicates that above the isostatic point the network rigidity dominates the non-affine response. Only if $T^{*}>10^{-4}$, we see that thermal fluctuations affect the non-affine response, increasing $\Gamma_{\text {mech. }}$ This is in contrast to fibre networks, where the non-affinity decreases with an increase in bending rigidity. We hypothesize that this difference occurs because in the case of fibre networks the fibres have a preference to remain straight to minimize stress caused by fibre bending, while in the case of thermal fluctuations an affine displacement of the nodes will not minimize the stress caused by the randomly oriented thermal fluctuations. Below the isostatic point, the effect of thermal fluctuations on the non-affine response is significant. We observe that at $T^{*}=10^{-8}$ the non-affine response is the smallest and that a moderate increase in $T^{*}$ up to $T^{*}=10^{-6}$ leads to an increase in the non-affine response, corresponding to what is observed for fibre networks. However, we also observe a decrease in $\Gamma_{\text {mech }}$ if the temperature is increased beyond $T^{*}=10^{-6}$, which is not observed in fibre networks. It is unclear if this deviation is caused by a fundamental difference between thermal fluctuations and bending rigidity as a stabilizing field or that longer equilibration times are required to gain quantitative information on the non-affine response in this regime (see Figure 3.A.1 and Figure 3.A.2 for details).

In general, we find that at a global level thermal fluctuations act as a stabilizing field in central-force spring networks, dampening rigidity-dependent behaviour around the isostatic point. However, our results suggest that the random nature of the thermal fluctuations causes significant differences in the local response with respect to stabilizing fields in athermal systems such as bending.

\subsubsection{The effect of thermal fluctuations on non-linear elasticity}

In the previous section, we have shown that thermal fluctuations rigidify subisostatic networks. Here, we analyze the non-linear elasticity of unbreakable networks to quantify the effect of temperature when networks become more and more strained. The strain-stiffening observed for subisostatic networks in the athermal limit has been extensively studied 6,11,18,19,33,34,34-36. In Figure 3.3(a), we report the stress-strain curves for a network with $p=0.56$ at different temperatures. It is evident that the network strain-stiffens for all the $T^{*}$ investigated. We observe that the onset of strain-stiffening is barely dependent on temperature. Furthermore, the stress response becomes independent of $T^{*}$ at high strains, similarly to what has been observed for other stabilizing fields, e.g. bending ${ }^{11,19}$.

Signatures of strain-stiffening can also be observed in the non-affine response of the network. In Figure 3.3(b), we report the instantaneous non-affinity parameter 
$\Gamma$, that intrinsically includes both the non-affine contributions from instantaneous thermal fluctuations and structural rearrangements. As a result, high non-affinity values can be observed at low strains, where the size of the non-affine thermal

(a)

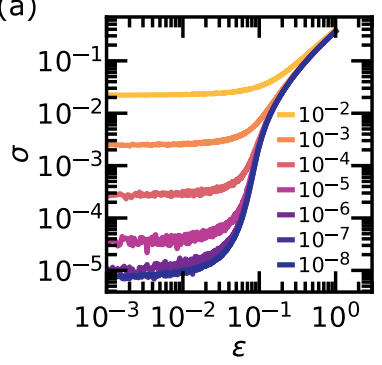

(c)

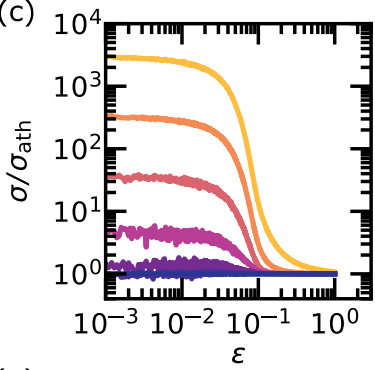

(e)

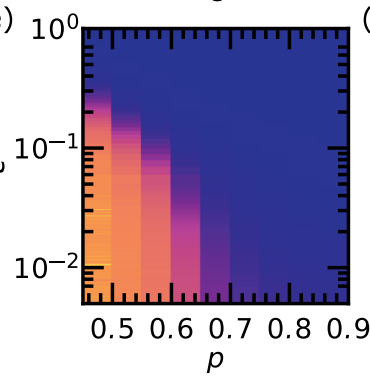

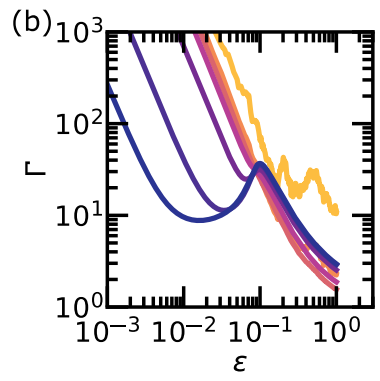

(d)

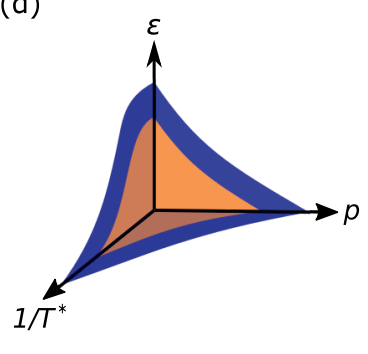

(f)

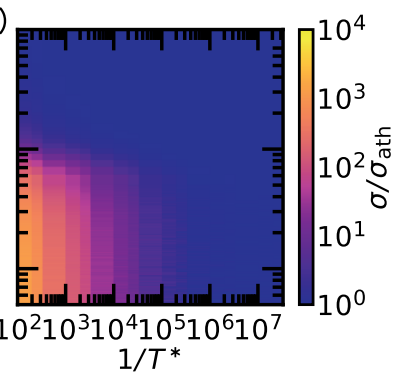

Figure 3.3. The role of temperature on the non-linear elasticity of unbreakable spring networks with fixed system size $L=128$. (a) Stress-strain curves for $p=0.56$ and several $T^{*}$ (indicated in the legend). (b) Instantaneous non-affinity as a function of strain. (c) The stress ratio $\sigma / \sigma_{\text {ath }}$ as a function of strain for different reduced temperatures $T^{*}$ with $\sigma_{\text {ath }}$ the stress measured in the athermal limit (practically, for $T^{*}=10^{-8}$ ). (d) Schematic mechanical phase diagram based on the stress ratio with two regimes: a temperature dominated regime (orange) and a mechanically dominated regime (blue). The gradual transition between the regimes depends on deformation $\varepsilon$, network connectivity $p$, and temperature $T^{*}$. (e-f) Two cross-sections of the diagram based on the simulations: (e) $\varepsilon-p$ plane for $T^{*}=10^{-3}$ and (f) $\varepsilon-1 / T^{*}$ plane for $p=0.56$. Every data point is based on simulations of at least 10 independent configurations. 
fluctuations is large compared to the applied strain. At low temperatures, a peak can be observed in the non-affine response around the onset strain. At high temperatures, this peak is overshadowed by the non-affine thermal fluctuations. At high strain, the network elasticity is controlled by stretching of the bonds, and the network response becomes increasingly affine for most temperatures. Only at $T^{*}=10^{-2}$, the non-affine fluctuations are still visible.

To disentangle the effects of temperature and network connectivity, we normalize the stress-strain curve with the ones obtained in the athermal energy-dominated limit. In particular, we plot the stress ratio $\sigma / \sigma_{\text {ath }}$ in Figure 3.3(c), where we used the data obtained at $T^{*}=10^{-8}$ for $\sigma_{\text {ath }}$. At this temperature the network behaves according to a network in the athermal limit, but still a small amount of stress is observed, i.e. the stress is not zero at small strains even below the isostatic point. A ratio of $\sigma / \sigma_{\text {ath }} \approx 1$ implies that the mechanical behaviour is basically insensitive to variations in temperature. As can be seen in Figure 3.3(c) for a network with $p=0.56$, there is a regime of strain in which the stress ratio depends on $T^{*}$ that decreases upon stretching the network more and more. At increasing temperature, this stress ratio is both higher at the start and approaches temperature insensitivity at a higher strain. The start of decrease in stress ratio for all temperatures occurs at approximately the same strain value, corresponding to the onset of strain-stiffening. This transition could therefore be interpreted as a transition between a regime dominated by thermal fluctuations to a regime dominated by bond stretching. This is analogous to the bending-to-stretching transition observed in fibre networks ${ }^{11,33,37}$. We summarize these observations in a mechanical phase diagram sketched in Figure 3.3(d), where we can distinguish two regimes: a mechanically-dominated regime (blue) where structural rigidity overpowers the effect of thermal fluctuations and a temperature-controlled regime (orange) where thermal fluctuations play a more important role in the elastic behaviour. The transition between these regimes depends on the reduced temperature $T^{*}$ (and therefore both on the actual temperature $T$ and the bond stiffness $\mu$ ), the connectivity parameter $p$ and the strain $\varepsilon$. This transition is in general very gradual as can be seen in the two cross-sections of the mechanical phase diagram reported in Figure 3.3(e-f) where we show the stress ratio obtained by some of our simulations. When $T^{*}$ is fixed (Figure 3.3(e)) and we increase $p$, we observe a steep decrease in the strain associated to the thermal-stretching transition. Above the isostatic point, the mechanics of the rigid networks is barely affected by thermal fluctuations at this temperature. In Figure 3.3(f), we observe that with increasing temperature the stress ratio increases but the strain characterizing the transition seems to reach a limiting value. This limiting value is a result of the onset of 
strain-stiffening, which is independent of temperature and corresponds to the transition to the elastic regime.

In summary, we identified a rigidity-dependent transition between two regimes where thermal fluctuations are or are not important. In the following sections, we will investigate whether this underlying transition also influences the fracture of these elastic networks.

\subsubsection{The effect of thermal fluctuations on macroscopic fracture}

Under athermal conditions, bonds break only after the onset strain, as only at this stage the bonds are under tension. Furthermore, Figure 3.3 indicates that the contribution of the thermal fluctuations to the stress in the system is significantly
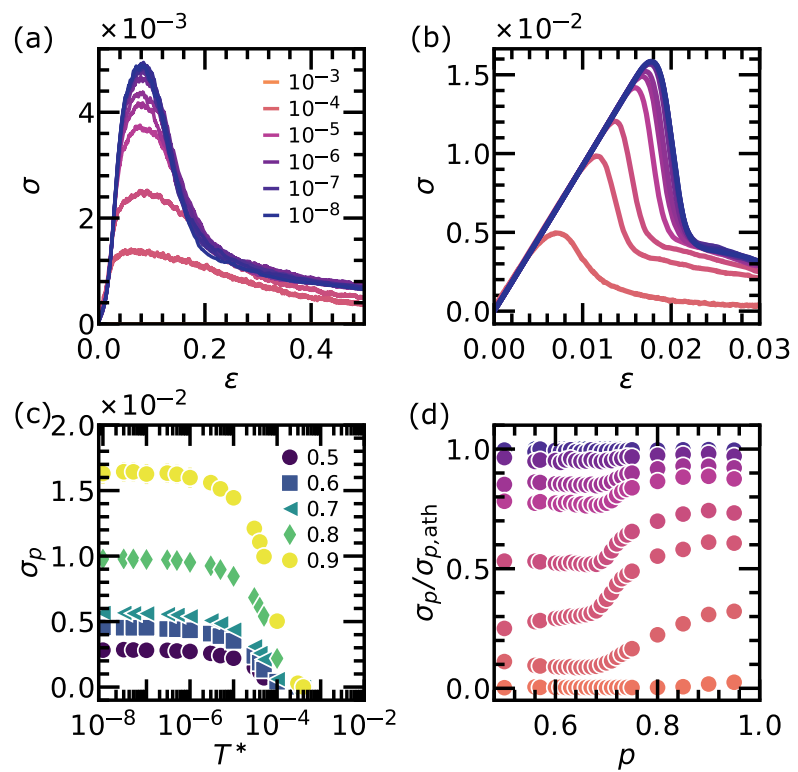

Figure 3.4. Stress-strain curves from fracture simulations for networks with $\lambda=0.03, L=128$ at different $T^{*}$ (see legend) and (a) $p=0.65$, (b) $p=0.90$. Average over 10 independent configurations, standard error falls within the width of the line. (c) Temperature dependence of the peak stress $\sigma_{p}$ for networks with different connectivity $p$ (values indicated in the legend). Every data point is based on simulations of at least 60 independent configurations. (d) Connectivity dependence of $\sigma_{p}$ normalized by the peak stress in the athermal limit $\sigma_{p}$, ath for several temperatures, same colour code as panel (a). Every data point is based on simulations of at least 10 independent configurations. 
reduced beyond the onset strain. Does this mean that there is only a minor influence of temperature on the failure response?

We first focus on macroscopic descriptors and characterize the stress-strain curves obtained from fracture simulations. In Figure 3.4(a-b), we show the response of two representative networks with a small rupture threshold $\lambda=0.03$ and different connectivities at several temperatures $T^{*}$. For the network with $p=0.65 \simeq p_{\text {iso }}$ (panel a), a clear decrease in peak stress $\sigma_{p}$ for increasing $T^{*}$ is observed, while a variation in the peak strain $\varepsilon_{p}$ is less evident as the fracture becomes more ductile and the decrease in stress after the peak is less pronounced. For the very rigid network ( $p=0.90$, panel $\mathrm{b})$, the decrease in both $\sigma_{p}$ and $\varepsilon_{p}$ is clearly observed. Similarly, the fracture becomes more ductile for higher $T^{*}$, even though a clear stress drop is still recognizable at the highest temperature simulated. In both cases, the networks become weaker with increasing $T^{*}$. Furthermore, when approaching the athermal limit $\left(T^{*} \rightarrow 0\right)$ the peak stress becomes less sensitive to variation in temperature. In Figure 3.4(c), we show the temperature dependence of $\sigma_{p}$ for several connectivities with $\lambda=0.03$. The common trend is little variation at low temperatures, almost a plateau that is indicative of approaching the athermal limit, followed by a decrease when temperature is increased, with $\sigma_{p}$ eventually dropping to zero when a temperature of $T^{*} \simeq 10^{-4}$ is reached. On the one hand, for low $T^{*}$ the peak stress is evidently controlled by the network rigidity, as previously investigated in the athermal limit ${ }^{12,13}$. On the other hand, when the thermal energy is of the order of $E_{\text {frac }}=\frac{1}{2} \mu\left(\lambda \ell_{0}\right)^{2}$ the network structure is irrelevant, as springs spontaneously break and the system shows melting behaviour. We will later describe the melting point using the reduced quantity $E_{\text {therm }} / E_{\text {frac }}=k_{\mathrm{B}} T /\left[\frac{1}{2} \mu\left(\lambda \ell_{0}\right)^{2}\right]=T^{*} /\left(\frac{1}{2} \lambda^{2}\right)$. In between these limits, there is a broad cross-over regime. To better assess the role of rigidity in this intermediate regime, we normalize $\sigma_{p}$ by its value in the athermal limit $\sigma_{p \text {,ath }}$ and plot this ratio in Figure 3.4(d). The transition between the athermal limit, where $\sigma_{p} / \sigma_{p \text {,ath }}=1$, and the melting limit, where such a ratio goes to zero, depends on a subtle coupling between connectivity and temperature itself. Far below $(p<0.60)$ and far above the isostatic point $(p>0.80)$ the connectivity plays a small role since at every temperature the curve exhibits two plateaus (at small and large $p$ ). However, around the isostatic point rigidity and thermal fluctuations are coupled, since at all the intermediate temperatures we can observe a sharp increase in $\sigma_{p} / \sigma_{p, \text { ath }}$ upon increasing $p$, connecting the two limiting plateaus. On passing, we note that the plateau for small $p$ is lower, suggesting that temperature starts to affect failure of very diluted networks earlier than for networks with large $p$. Furthermore, we speculate that the complex temperature-dependence around the isostatic point arises from locally floppy regions that are rigidified by thermal 
fluctuations (whose magnitude depends on temperature itself) and are therefore able to sustain and concentrate stress, and break. Since the isostatic point marks the onset of mechanical stability, such effect is largest for networks close to it.

\subsubsection{The effect of thermal fluctuations on microscopic fracture}

Clearly, the failure response is temperature dependent across the entire connectivity range, but the influence of temperature indeed seems to depend on the distance with respect to the isostatic point i.e. on the network rigidity. Are these differences also apparent at the microscopic level? To investigate this, we monitor the number of broken bonds during the simulations. As shown in Figure 3.5(a-b), the fraction of broken bonds $\phi$ as a function of deformation indicates that higher temperature

(a)

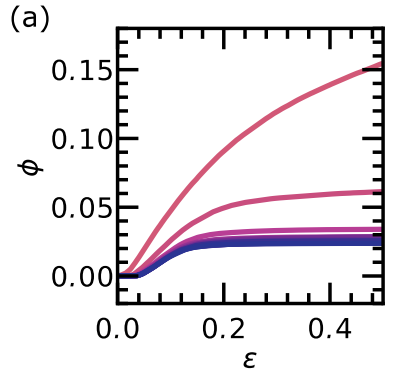

(c)

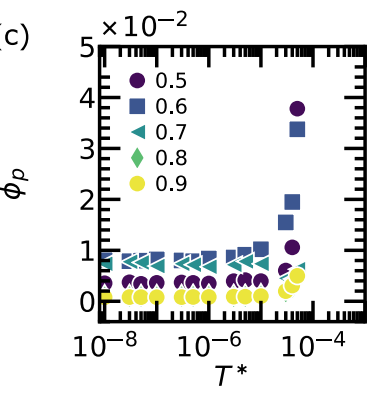

(b)
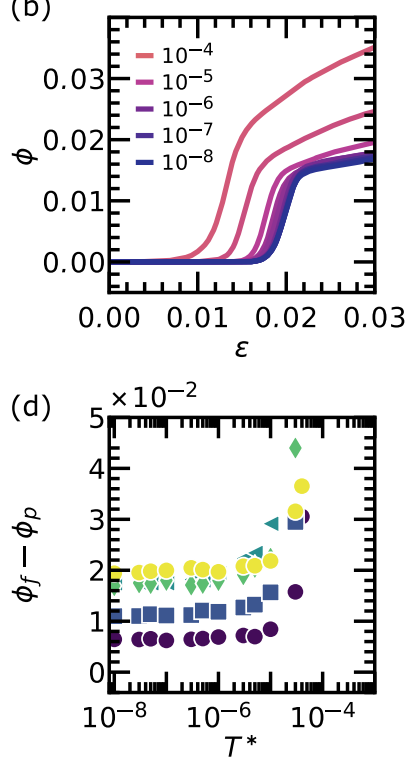

Figure 3.5. Effect of temperature on the development of microscopic damage. (a-b) Fraction of broken bonds $\phi$ as a function of strain for networks with $L=128, \lambda=0.03$ and (a) $p=0.65$, (b) $p=0.90$. Average over 10 independent configurations, standard error falls within the width of the line. (c) Fraction of broken bonds at the peak strain $\phi_{p}$ (including the peak event) as a function of $T^{*}$ for a range of dilution factors $p=0.50-0.90$. Every data point is based on simulations of at least 60 independent configurations. (d) Fraction of broken bonds after the peak strain up to failure of the entire system as a function of $T^{*}$ for a range of dilution factors $p=0.50-0.90$. Data are only shown for systems that lose percolation during the simulations (before $100 \%$ strain). All fractions are calculated with respect to the initial number of bonds in the diluted network. 
leads to earlier and overall increased damage. However, the effect of temperature is more significant close to the melting temperature $T^{*} \simeq \frac{1}{2} \lambda^{2}=4.5 \times 10^{-4}$, whereas at lower temperatures the system response is still highly influenced by rigidity. By focusing on the fraction of bonds broken at the peak strain $\phi_{p}$, counting also the bonds broken during the peak event, as shown in Figure 3.5(c), the diverging behaviour when approaching melting is evident. This increase in broken bonds could explain the decrease in material strength $\sigma_{p}$. The fraction of broken bonds observed here is of the same order of magnitude as the fraction of broken bonds observed in literature for athermal systems where all elements have equal strength $^{12,13,21}$. Also the fraction of bonds that break above $\varepsilon_{p}$ (Figure 3.5(d)), the post-peak response, increases close to the melting point, which points towards a prolonged post-peak response, i.e. higher ductility.

A direct inspection of the simulation snapshots (Figure 3.6) suggests that bonds that break up to the peak strain $\varepsilon_{p}$ (red bonds) are dispersed more homogeneously throughout the sample at a higher temperature. The snapshots also reveal a big difference in the response to temperature between networks around $(p=0.65)$ and far above the isostatic point $(p=0.90)$. Around the isostatic point, the damage up to $\varepsilon_{p}$ is already diffusive in the athermal limit, and its delocalization is enhanced when the temperature is increased. In contrast, the failure response far above the isostatic point shows a clear transition from crack nucleation in the athermal regime to a more diffuse failure response close to the melting point. However, the post peak response at $p=0.90$ is clearly still dominated by the propagation

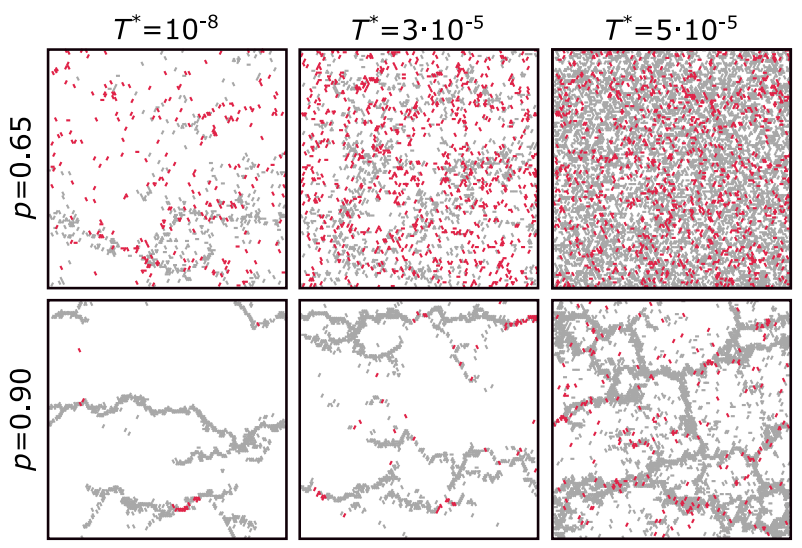

Figure 3.6. Failure patterns are presented as snapshots of the networks $(L=128)$ in their rest state, only showing broken bonds. The bond colour indicates whether the bond was broken before (red) or after (grey) $\varepsilon_{p}$. 
of cracks. Nevertheless, at high temperatures we observe the development of multiple cracks, sometimes even not perpendicular to the deformation direction, and evidence of crack merging.

In summary, we show that an increase in temperature leads to an increase in diffuse failure, implying suppression of stress concentration before the peak stress. These observations suggest that thermal fluctuations are responsible for two apparently contrasting effects: on the one hand, they create "instantaneous defects" resulting in more regions with broken bonds, that reduce material strength; on the other hand, the fluctuations allow the material to delocalize stress away from such defects, delaying the propagation of large cracks. As a result, the damage pattern is diffuse throughout the system.

\subsubsection{Thermal fluctuations increase the length scale of stress redistri- bution}

In the last section we have interpreted the effect of thermal fluctuations on the microscopic failure mechanisms in terms of defects and stress concentration. However, it should be realized that the use of these terms in (spring) networks comes with more challenges than the use of these terms in a continuum description of a material. In a disordered network, it proves challenging to identify defects, because the edges of a large void do not necessarily coincide with locations of stress concentration. Moreover, we observe that in networks stress can be redistributed over a large part of the network via non-affine rearrangements of the network. As a result, both the concentration of stress and the failure response in spring networks are dependent on the size of the network. It might therefore be possible to identify a characteristic length scale associated to stress concentration (or to stress delocalization) in these spring networks. For example, we have recently ${ }^{13}$ shown that in athermal systems, brittle (abrupt) fracture always occurs for networks above a certain system size $L^{*}$. This critical size can be tuned by the network rigidity, i.e. by varying $p$ and $\lambda$. The onset of the size-induced brittleness can be determined by looking at the non-monotonic size-dependence of $\Delta \sigma_{\max }$. Here, we investigate whether temperature affects this critical system size, which can be interpreted as a characteristic length scale at which the concentration of stress can be observed. This analysis is therefore another way to further assess the role of temperature on stress concentration.

Therefore, we examine how thermal fluctuations affect the macroscopic fracture descriptors for different system sizes, focusing on the maximum stress drop $\Delta \sigma_{\max }$ that quantifies fracture abruptness. In Figure 3.7(a-d), we plot the size-scaling of the maximum stress drop $\Delta \sigma_{\max }$ (closed symbols) together with the peak stress 
$\sigma_{p}$ (open symbols) for four combinations of $p$ and $\lambda$ at different temperatures. In all cases, we observe a monotonic decrease of $\sigma_{p}$ as a function of the system size. These trends can be fitted by a power law $\sigma_{p}=(L / \alpha)^{-\beta}+\sigma_{p}^{\infty}$, where $\sigma_{p}^{\infty}$ is the failure stress in the thermodynamic limit (infinite system size), $\beta$ the size scaling exponent and $\alpha$ a fitting constant. Values for $\beta$ are comparable to values found in literature ${ }^{13,22}$. It is interesting to note that we find a finite value for $\sigma_{p}^{\infty}$, which is different from many other studies on network failure ${ }^{38}$. This is because in our work all elements have the same strength and therefore a finite amount of stress is required at all network sizes to start the failure process. In contrast, some studies reported a vanishing $\sigma_{p}^{\infty}$ since they employed a distribution in element strength extending to zero ${ }^{38}$. In Figure 3.7(e), we plot $\sigma_{p}^{\infty}$ normalized
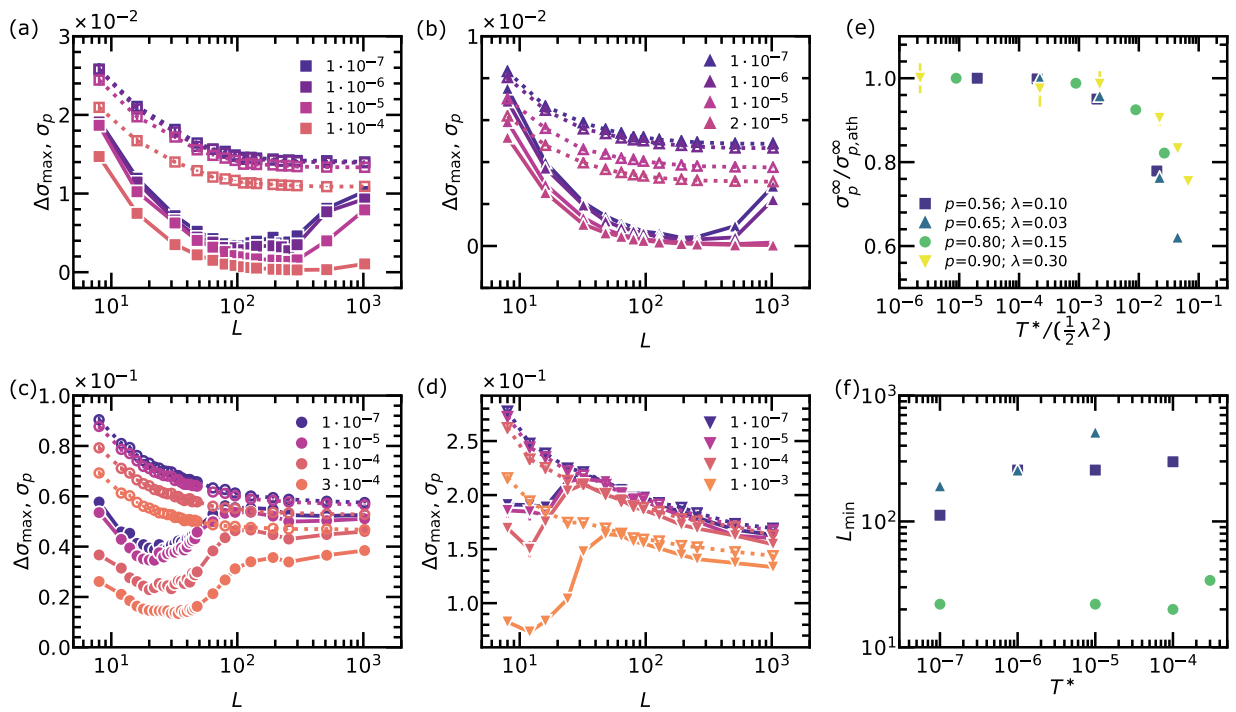

Figure 3.7. Size-scaling and temperature. (a-d) Maximum stress drop $\Delta \sigma_{\max }$ (closed symbols, solid lines) and peak stress $\sigma_{p}$ (open symbols, dotted lines) as a function of system size $L$ for systems with (a) $p=0.56$ and $\lambda=0.10$ (square), (b) $p=0.65$ and $\lambda=0.03$ (triangle), (c) $p=0.80$ and $\lambda=0.15$ (circle), and (d) $p=0.90$ and $\lambda=0.30$ (downward triangle). Depending on system size the minimum number of independent simulations per data point is 60 ( $L=8 \ldots 128)$, $30(L=192 \ldots 256), 10(L=512)$ or $5(L=1024)$. (e) Peak stress in the thermodynamic limit $\sigma_{p}^{\infty}$ normalized by the corresponding value in the athermal limit $\sigma_{p \text {, ath }}^{\infty}$ as a function of reduced temperature $T^{*}$ normalized by its melting value $\frac{1}{2} \lambda^{2}$. Error bars represent the standard error in the fit for $\sigma_{p}^{\infty}$. (f) Estimate of the system size where $\Delta \sigma_{\max }$ is minimal as a function of $T^{*}$. In both (e) and (f) the marker shape corresponds to the value of $p$ as introduced in panels (a-d). 
by its athermal value $\sigma_{p, \text { ath }}^{\infty}$ as a function of $T^{*}$ normalized by $\frac{1}{2} \lambda^{2}$ (see Figure 3.A.7 for the other fitting parameters). The observed trend underlying a transition from low $T^{*}$ to melting is consistent with the data at fixed system size and fixed rupture threshold $\lambda$ presented earlier in Figure 3.4. Here, we can also appreciate the effect of varying $\lambda$ in the intermediate temperature regime. For example, the largest $\lambda=0.30$ (downward triangles, networks with $p=0.90$ ) shows a steeper decrease in the normalized fracture stress, suggesting that thermal effects kick in at higher temperatures for these very rigid networks.

Finally, we focus on the maximum stress drop $\Delta \sigma_{\max }$. From Figure 3.7(a-d), we observe that a non-monotonic trend is observed in basically all cases, consistent with our previous results in the athermal limit ${ }^{13}$. We speculated that the initial decrease, implying a more ductile fracture upon increasing system size, is associated to the rupture and reformation of locally stressed regions (often consisting of aligned springs, and sometimes called force chains ${ }^{12,39-41}$ ). However, upon increasing the system size $\Delta \sigma_{\max }$ starts to increase, suggesting that stress concentration around defects is present in the system, since it fractures in a more abrupt way. At even larger $L, \Delta \sigma_{\max }$ decreases again, now following the same trend for the peak stress $\sigma_{p}$ that sets the upper bound to the possible stress drop. In Figure 3.7(c) the entire trend is visible for the system sizes explored in this work, whereas in the other panels only parts of it are captured. Importantly, for all systems, the trend depends on temperature. In particular, in Figure 3.7(f) we quantify the effect of temperature by plotting the system size $L_{\min }$ corresponding to the minimum $\Delta \sigma_{\max }$ as a function of $T^{*}$. We observe that thermal fluctuations increase the value of $L_{\min }$, which can be interpreted as a length scale for stress concentration. The role of temperature seems particularly relevant at low connectivity, where the stress is already very delocalized in the athermal limit.

In summary, we find that also in the thermodynamic limit there is a crossover from an athermal regime to a melting regime where the failure behaviour is determined by both rigidity and thermal fluctuations. Moreover, thanks to the analysis of $\Delta \sigma_{\max }$, we find evidence that temperature increases the region over which stress is delocalized.

\subsubsection{Thermal fluctuations homogenize stress}

The delocalization of stress is mediated by structural rearrangements in the network. Therefore, if temperature helps to delocalize stress as suggested by Figure 3.6 and Figure 3.7(f), this must be evident in the distribution of stress within the network. In Figure 3.8(a-b) we show two snapshots of the same deformed network $(p=0.65)$ at two different temperatures, together with the 
associated histogram of the bond deformation 3.8(c-d), which is equivalent to the probability distribution of the microscopic stresses since the springs are linear. Both networks are deformed up to $10 \%$ strain, which is close to $\varepsilon_{p}$ and well above the onset strain discussed in Section 3.3.2. At the lower temperature, the stress is distributed very heterogeneously, as indicated by an asymmetric distribution with an exponential tail containing few bonds carrying a high load. The regions of high stress, typically composed of aligned highly-stressed bonds, that we call force chains, can be readily identified in the simulation snapshot. On the contrary, for the higher temperature, the distribution is symmetric, resembling a Gaussian distribution, and the force chains can not be identified. This indicates that even above the onset strain thermal fluctuations act as a stabilizing field as discussed in Section 3.3.1 and do affect the distribution of stress in the network. To quantify

(a)

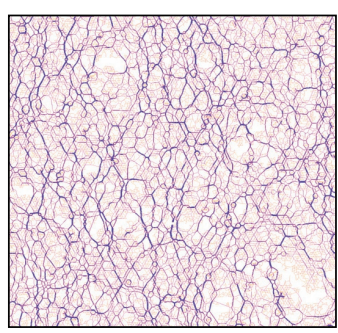

(c)

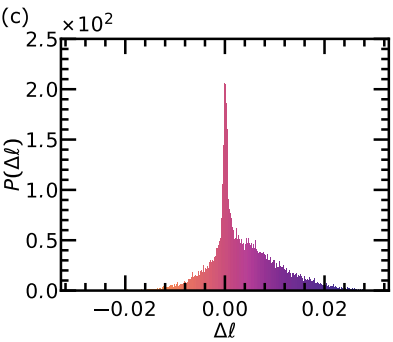

(b)

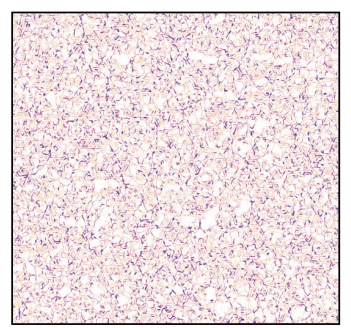

(d)

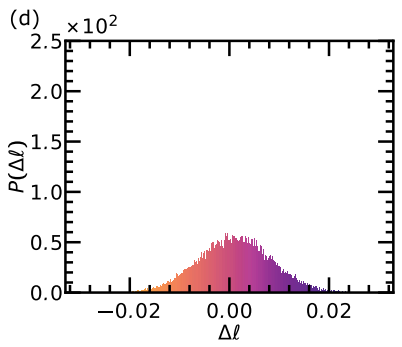

(e)

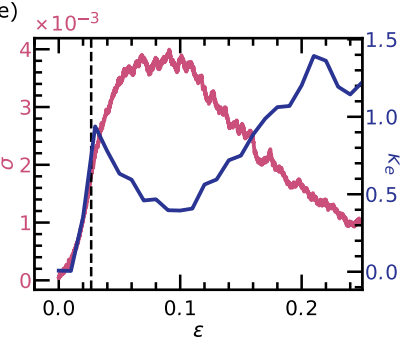

(f)

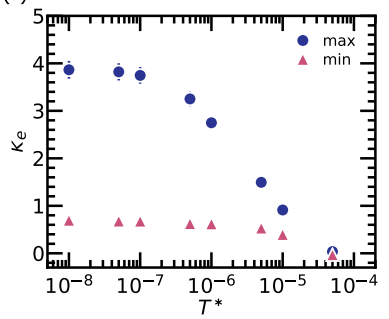

Figure 3.8. Influence of temperature on the stress distribution in networks with $p=0.65, \lambda=$ 0.03 and $L=128$. (a-b) Snapshots of the local bond extension at $10 \%$ strain and (c-d) the corresponding histograms. The colour scale indicates the amount of bond extension (i.e. local stress) on the network bonds, purple corresponds to high stress and orange to low stress. (a) For $T^{*}=10^{-7}$, aligned highly-stressed bonds ("force chains") are visible. (b) For $T^{*}=5 \times 10^{-5}$, the stress distribution is highly homogeneous. (e) Stress and excess kurtosis $\kappa_{e}$ of the stress histogram, a measure of heterogeneity, as a function of the strain for a single simulation. The dashed black line indicates the strain at which the first bond breaks, also corresponding to the maximum of $\kappa_{e}$; whereas the minimum of $\kappa_{e}$ occurs at the peak stress. (f) Maximum and minimum of $\kappa_{e}$ as a function of $T^{*}$. Every data point is based on simulations of 10 independent configurations. 
this heterogeneity, we calculate the excess kurtosis $\kappa_{e}$ of the stress distribution, an indicator of the tail heaviness of a distribution (being zero for a Gaussian). This measure has been recently used to quantify stress heterogeneities in porous materials ${ }^{42}$. To illustrate how the heterogeneity of the stress distributions is linked to the macroscopic stress evolution, in Figure 3.8(c) we plot both $\kappa_{e}$ and $\sigma$ as a function of strain for an example simulation run. As observed in most cases, the strain-stiffening of the network is accompanied by a similar increase in kurtosis. The stress distribution becomes more heterogeneous until (approximately) the first bond breaks (the dashed black line in Figure 3.8(c)), after which strain softening occurs and the stress distribution becomes more homogeneous. This decrease in heterogeneity is presumably caused by redistribution of the stress after bonds are broken. Strikingly, in correspondence with the peak stress, a local minimum for the kurtosis is observed. The subsequent stress drops are instead accompanied by an increase in $\mathcal{K}_{e}$, and therefore in the microscopic stress heterogeneity. This increase indicates stress concentration somewhere in the network leading to significant bond breakage that does not allow for a larger stress response. To show how the stress heterogeneity changes with temperature, we plot the maximum and the minimum of $\kappa_{e}$ as a function of $T^{*}$ in Figure 3.8(d). To determine the minimum kurtosis, we take the smallest value of $\kappa_{e}$ in a strain interval close to the peak strain $\varepsilon_{p}$, to avoid lower values that might be found before strain-stiffening. Analogously, for the maximum kurtosis, we only look at the maximum up to and including the peak strain, to avoid post-peak values. Both quantities clearly decrease when the temperature is raised, but follow different curves. In particular, the maximum of $\kappa_{e}$, that is associated to the network strain-stiffening, is immediately sensitive to temperature changes, in line with our previous observations on the non-linear elasticity (Section 3.3.2), while the minimum of $\kappa_{e}$, associated to the fracture peak, exhibits an initial temperature insensitive interval, similarly to the other fracture descriptors investigated above. Furthermore, as the temperature increases, the difference between the maximum and minimum becomes smaller, and eventually both quantities reach zero (homogeneous stress distribution) at the melting temperature. Note that, while we have shown here results only for a given connectivity, the fact that a higher temperature allows for better redistribution of the stress during fracture was a consistent observation in our simulation study.

\subsection{Concluding remarks}

In this work, we explored the relation between rigidity and the failure of centralforce spring networks under the influence of thermal fluctuations. Our results demonstrate that thermal fluctuations couple with network rigidity and affect the 
non-linear mechanics of elastic networks. In general, thermal fluctuations lead to a lower failure strength (Figure 3.4), an increased ductility, and an increased fraction of broken bonds (Figure 3.5). We have shown that at the microscopic level the failure response is altered with respect to the athermal case in two ways: i) bond failure can be activated by instantaneous thermal fluctuations, creating additional weak spots, and ii) stress is delocalized, suppressing the expansion of existing defects (Figure 3.6). We reveal that temperature acts as a stabilizing field that resists large structural non-affine deformation within the network (Figure 3.2 and Figure 3.3). Specifically, the thermal fluctuations increase the length scale over which stress is redistributed, which can be quantified via the maximum stress drop (Figure 3.7) and the excess kurtosis (Figure 3.8). Although these trends can be observed for all connectivities, there are distinct damage mechanisms above and below the isostatic point. Above the isostatic point, the failure up to the peak stress shifts from crack nucleation at a single site to a more diffuse failure pattern, while around the isostatic point the failure response is already delocalized in the athermal limit and the fraction of broken bonds is enhanced approaching the melting point (Figure 3.6). These distinct failure processes might explain the difference in how the peak strain depends on temperature with respect to rigidity (Figure 3.4).

We note that at a first glance central-force spring networks subjected to thermal fluctuations behave like athermal networks in a stabilizing field. However, the instantaneous nature of the thermal fluctuations introduces important differences. It is striking that, without any applied deformation, the thermal fluctuations induce structural rearrangements of the average network structure (Figure 3.A.1). Furthermore, providing enough time, the thermal fluctuations allow the failure of bonds even if they are not intrinsically under tension (activated failure), leading to diffuse damage. A final consequence of introducing thermal fluctuations is that time becomes an important parameter. In our simulations the system was deformed at a constant strain rate, i.e. it was driven at a given speed. If the driving speed is too low, the system will melt due to the process of activated failure. If the driving speed is too high, the system has no time for stress relaxation as it is held back by the viscous surroundings. Therefore, the failure response of an elastic network is generally determined by the coupling between the driving speed, viscosity, rigidity and thermal fluctuations. Our study was focused on a regime in which driving and viscosity effects were small (see Appendix for discussion).

This work provides new insight into the relation between the static network structure, thermal fluctuations and the failure response of central-force spring networks. Above all, it shows that rigidity remains a controlling parameter in the failure response of spring networks in the presence of thermal fluctuations, even 
close to the melting temperature. This suggests that the failure response of thermal networks in experiment, such as semiflexible polymer networks, could be rigiditydependent as well. The ratio between the energy of the thermal fluctuations, $E_{\text {therm }}=k_{\mathrm{B}} T$, and the energy required to break an elastic element, $E_{\mathrm{break}}$, emerges as a relevant parameter to classify the failure regime of a particular network (either the athermal regime, the cross-over regime, or the melting regime) and could thus be a relevant parameter to classify the failure response of experimental systems. For example, a rough estimate of this ratio for experimental systems $E_{\text {therm }} / E_{\text {break }}=T^{*} /\left(\frac{1}{2} \lambda^{2}\right)$ shows us that for a collagen network $E_{\text {therm }} / E_{\text {break }} \approx$ $1 \cdot 10^{-6}$, which corresponds to the athermal limit, while the values for a semiflexible network like actin $\left(E_{\text {therm }} / E_{\text {break }} \approx 8 \cdot 10^{-3}\right)$ and a flexible polymer network $\left(E_{\text {therm }} / E_{\text {break }} \approx 7 \cdot 10^{-3}\right.$ ) are significantly higher (see Appendix for details). These examples show that it is not just the temperature, but also the type of building block that determines the relevant failure regime. Furthermore, the ratio also makes clear that the temperature sensitivity is not only dependent on the element stiffness $\mu$, but also on extensibility $\lambda$. Therefore, it is a possibility that a network with a temperature dependent elastic response at the network level (determined by $T^{*}$ ), might not be sensitive to thermal fluctuations in the failure regime (determined by $T^{*} /\left(\frac{1}{2} \lambda^{2}\right)$ ). Our model predicts that networks with weak crosslinkers, i.e. small $\lambda$, are most likely to have a failure response as observed in the cross-over regime.

We hope that our simulation results will stimulate further experimental work aimed at mapping out the roles that rigidity and thermal fluctuations play in governing mechanical failure of elastic networks. 


\section{A Appendix}

\section{A.1 Dependence of non-affinity on the reference coordinates}

We calculate the non-affine deformation with respect to the time averaged position at $0 \%$ strain (Figure 3.A.1(a)) $\overline{\boldsymbol{r}}_{0}$. Alternatively, the non-affinity can be calculated with respect to the initial positions of the nodes (located on a regular triangular lattice) $\boldsymbol{r}_{\text {init }}$ (Figure 3.A.1(b)). The difference between Figure 3.A.1(a) and Figure 3.A.1(b) indicates that temperature has an effect on the equilibrium node positions at $0 \%$ strain. This is in contrast with athermal networks and bending stabilized networks, where the equilibrium node positions are equal to the initial position of the nodes. Figure 3.A.1(c) shows that the average displacement of the nodes from their initial position to their equilibrium position $d r=\left\langle\left|\overline{\boldsymbol{r}}_{0}-\boldsymbol{r}_{\text {init }}\right|\right\rangle$ depends on both temperature and connectivity. Especially below the isostatic point there are significant reorganizations within the network. However, at high temperatures also the network structure well above the isostatic point is affected. Clearly, the thermal fluctuations do affect the equilibrium structure at $0 \%$ strain.
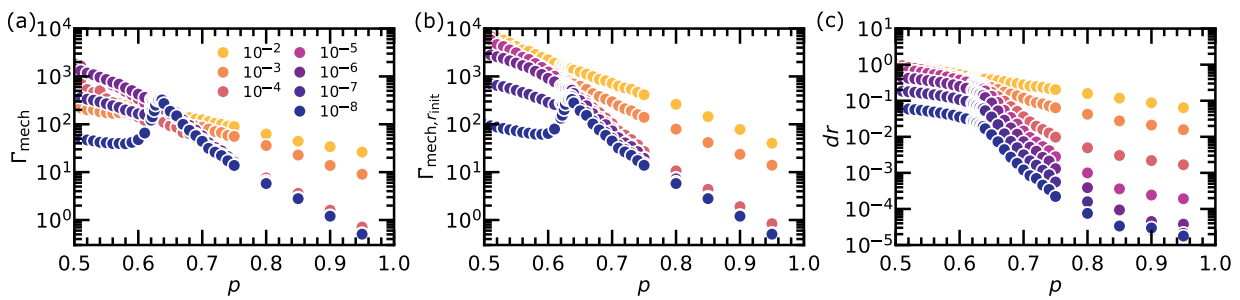

Figure 3.A.1. Non-affine response in the linear regime versus $p$ for a range of $T^{*}$ (colours are indicated in the legend). (a) The time-averaged (over 1900 $\tau$ ) non-affine response at $1.5 \%$ strain with respect to the time-averaged position of the nodes at $0 \%$ strain $\overline{\boldsymbol{r}}_{0}$. (b) The time-averaged non-affine response at $1.5 \%$ strain with respect to the coordinates $r_{\text {init }}$ of the initial configuration (a regular triangular lattice). (c) The ensemble averaged displacement $d r=\left\langle\left|\overline{\boldsymbol{r}}_{0}-\boldsymbol{r}_{\text {init }}\right|\right\rangle$ at $1.5 \%$ strain. Every data point is based on simulations of at least 10 independent configurations.

\section{A.2 Size of the thermal fluctuations}

To quantify the size of the thermal fluctuations, we monitor the root mean squared displacement of the nodes with respect to their equilibrium position $\sqrt{\left\langle\boldsymbol{u}_{\text {therm }}^{2}\right\rangle}$ and define the size of the fluctuations as $d r_{\text {fluc }}=\sqrt{\overline{\left\langle\boldsymbol{u}_{\text {therm }}^{2}\right\rangle}}$ with - representing a time-average. From Figure 3.A.2(a) it is clear that the size of the fluctuations of the nodes depends on both temperature and connectivity. In general, the size 
of the fluctuations decreases with an increase in connectivity, indicating there is feedback between the number of constraints imposed on a node in the network and how far the nodes can move. Before measuring $\sqrt{\overline{\left\langle\boldsymbol{u}_{\text {therm }}^{2}\right\rangle}}$, all systems are subjected to the same calibration run of $100 \tau$ (e Figure 3.A.2(b)/(c)). We note that for $p=0.65$ (Figure 3.A.2(b)) the required time to reach a stable value of $\sqrt{\overline{\left\langle\boldsymbol{u}_{\text {therm }}^{2}\right\rangle}}$ is longer for lower temperatures, indicating that the rate of equilibration depends on temperature. Furthermore, we see that at the higher connectivity value $p=0.90$ (Figure 3.A.2(c)) the fluctuations are smaller and reach their equilibrium value faster.
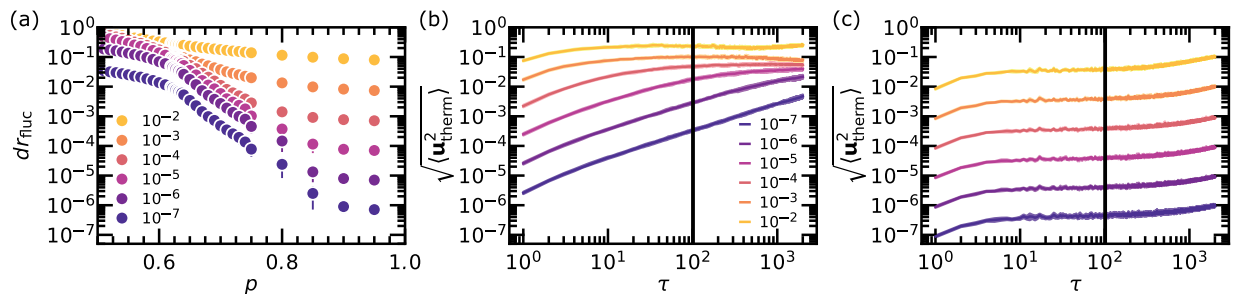

Figure 3.A.2. The size of the thermal fluctuations of the node positions. (a) the time-averaged (over $1900 \tau$ ) root mean squared fluctuation size $d r_{\text {fluc }}=\sqrt{\overline{\left\langle\boldsymbol{u}_{\text {therm }}^{2}\right\rangle}}$ versus $p$ for a range of $T^{*}$ (colours are indicated in the legend). (b-c) The development of $\sqrt{\left\langle\boldsymbol{u}_{\text {therm }}^{2}\right\rangle}$ as a function of time $\left(2000 \tau\right.$ in total). The black line is placed at $100 \tau$, time-averages for $\Gamma_{\text {mech }}$ and $\sqrt{\left\langle\boldsymbol{u}_{\text {therm }}^{2}\right\rangle}$ are based on data past this line. (b) $p=0.65$ and (c) $p=0.90$. Every data point is based on simulations of at least 10 independent configurations.

\section{A.3 Relation between time-averaged non-affinity and instantaneous non-affinity}

In an athermal elastic network the position of the crosslinks is determined by the applied deformation and the non-affine response of the nodes. In a thermal elastic network, the positions of the nodes are also influenced by thermal fluctuations of the nodes. The position of a node $r$ under uniaxial extension $\varepsilon$ can therefore be described as

$$
\boldsymbol{r}(\varepsilon, T, p)=\overline{\boldsymbol{r}}_{0}+\boldsymbol{u}_{\mathrm{aff}}(\varepsilon)+\boldsymbol{u}_{\text {naff }}(\varepsilon, T, p)+\boldsymbol{u}_{\text {therm }}(\varepsilon, T, p),
$$

where $p$ is the network connectivity parameter, and $T$ temperature. $\boldsymbol{u}$ stands for a displacement vector and $\overline{\boldsymbol{r}}_{0}$ is the time averaged position at $0 \%$ strain. If a system is fixed at a certain strain $\varepsilon$ the average position of the particle over time will be, 


$$
\overline{\boldsymbol{r}}=\overline{\boldsymbol{r}}_{0}+\boldsymbol{u}_{\mathrm{aff}}(\varepsilon)+\boldsymbol{u}_{\text {naff }}(\varepsilon, T, p),
$$

assuming $\left|\overline{\boldsymbol{u}}_{\text {therm }}\right|=0$. If we instead monitor the average size of the fluctuations of the nodes we find that

$$
\overline{(\boldsymbol{r}-\overline{\boldsymbol{r}})^{2}}=\boldsymbol{u}_{\text {therm }}^{2}(\varepsilon, T, p) .
$$

Note that in case of drift in the system center of mass, this needs to be taken into account. In our case, we assume $\langle\boldsymbol{r}\rangle=\boldsymbol{r}_{\text {com }}$. Below, we will detail how these contributions are related to the measure for non-affinity $\Gamma$.

\section{Non-affine response of time averaged positions}

We will start with the non-affinity based on time-averaged positions (Equation 3.6), as this parameter is directly related to the non-affinity parameter discussed for athermal systems that describes the size of non-affine rearrangements of the network.

$$
\Gamma_{\text {mech }}=\frac{\left\langle\left(\overline{\boldsymbol{r}}-\overline{\boldsymbol{r}}_{\mathrm{aff}}\right)^{2}\right\rangle}{\varepsilon^{2} \ell_{0}^{2}}=\frac{\left\langle\left(\boldsymbol{u}_{\text {naff }}\right)^{2}\right\rangle}{\varepsilon^{2} \ell_{0}^{2}} .
$$

To simplify the equation our definition for $\bar{r}$ is used (Equation 3.6) and the definition $\bar{r}_{\text {aff }}=\bar{r}_{0}+u_{\text {aff }}$.

\section{Non-affine response of instantaneous positions}

In a system with thermal fluctuations, the instantaneous positions of the node will also be determined by (non-affine) thermal fluctuations (see Equation 3.5). While monitoring the non-affinity during a continuous deformation, the non-affinity parameter $\Gamma$ will therefore include both the effects of non-affine rearrangements and thermal fluctuations.

$$
\Gamma=\frac{\left\langle\left(\boldsymbol{r}-\overline{\boldsymbol{r}}_{\mathrm{aff}}\right)^{2}\right\rangle}{\varepsilon^{2} \ell_{0}^{2}}=\frac{\left\langle\left(\boldsymbol{u}_{\mathrm{naff}}+\boldsymbol{u}_{\mathrm{therm}}\right)^{2}\right\rangle}{\varepsilon^{2} \ell_{0}^{2}} .
$$

\section{The relation between $\Gamma$ and $\Gamma_{\text {mech }}$}

An essential difference with respect to athermal networks is that $\Gamma$ is a result of both non-affine structural rearrangements and thermal fluctuations. Here we show the relation between $\Gamma_{\text {mech }}$ and $\Gamma$. We can rewrite the non-affinity such that we only have sums over all particles on the right hand side. 
$\left[\Gamma-\Gamma_{\text {mech }}\right] N \varepsilon^{2} \ell_{0}^{2}=\sum\left(\left(\boldsymbol{u}_{\text {naff }}+\boldsymbol{u}_{\text {therm }}\right)^{2}\right)-\sum\left(\left(\boldsymbol{u}_{\text {naff }}\right)^{2}\right)=\sum\left(\boldsymbol{u}_{\text {therm }}^{2}+2 \boldsymbol{u}_{\text {naff }} \cdot \boldsymbol{u}_{\text {therm }}\right)$.

Returning to the averages over all particles we can now distinguish a term related to thermal fluctuations and a cross-term related to both the non-affine deformation and the thermal fluctuations,

$$
\Gamma=\Gamma_{\text {mech }}+\frac{\left\langle\boldsymbol{u}_{\text {therm }}^{2}\right\rangle}{\varepsilon^{2} \ell_{0}^{2}}+\frac{2\left\langle\boldsymbol{u}_{\text {naff }} \cdot \boldsymbol{u}_{\text {therm }}\right\rangle}{\varepsilon^{2} \ell_{0}^{2}} .
$$

Hence, the contributions of structural rearrangements and thermal fluctuations to $\Gamma$ can not be decoupled.

\section{A.4 The influence of driving and viscosity}

The mechanical and failure response can depend on both the driving speed and the friction coefficient of the nodes, i.e., viscosity of the implicit solvent. In Figure 3.A.3 we show how these parameters affect the stress-strain curves around the isostatic point (Figure 3.A.3(a-b)) and far above the isostatic point (Figure 3.A.3(d-e)). An increase in either $\dot{\varepsilon}$ or $\zeta$ has a similar effect as both parameters affect the relaxation time of stress in the system. Furthermore, the friction coefficient controls the amount of damping in the system, from the underdamped regime at low $\zeta$, where inertia plays a role, to the overdamped (Brownian) regime at high $\zeta$, where the effect of inertia is negligible. In general, an increase in these parameters leads to an increase in ductility. Below the isostatic point, both the pre-peak and post-peak behaviour are affected, while above the isostatic point it is mostly the post peak response. Furthermore, we show the peak stress $\sigma_{p}$ as a function of the strain rate for $p=0.65$ and $p=0.90$ for a range of $\zeta$ and two values of $T^{*}: 1 \times 10^{-9}$ (purple) and $1 \times 10^{-5}$ (blue). We see that typically the peak stress increases with the strain rate. At a higher strain rate, there is less time for stress relaxation, leading to a more affine response and a higher stress in the system. Similarly, we observe that the peak stress increases with an increase in the friction coefficient, which is also related to the time for stress relaxation $\tau_{0}=\zeta / \mu$. In the main text, we set the strain-rate $\dot{\varepsilon}=0.001$ and the friction coefficient $\zeta=10$. We can observe that for $p=0.65$ we are in a regime where the effect of $\dot{\varepsilon}$ and $\zeta$ on the peak stress is relatively small. At $p=0.90$ the effect of the strain rate is bigger; however, we do not expect that this affects our conclusions. 
(a)

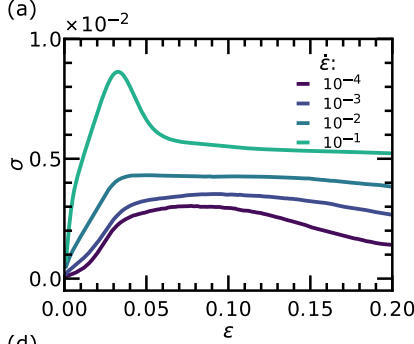

(d)

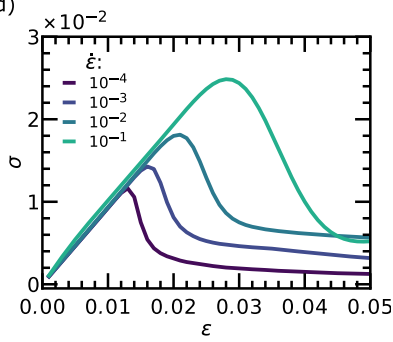

(b)

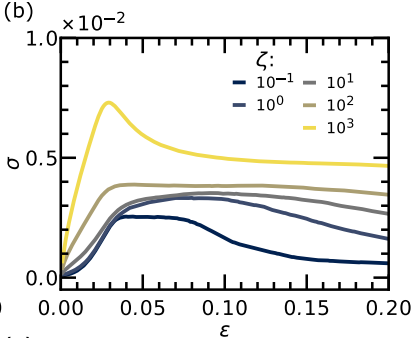

(e)

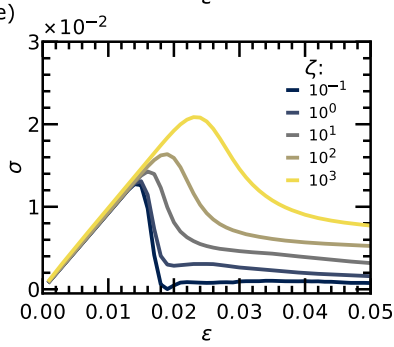

(c)

(f)
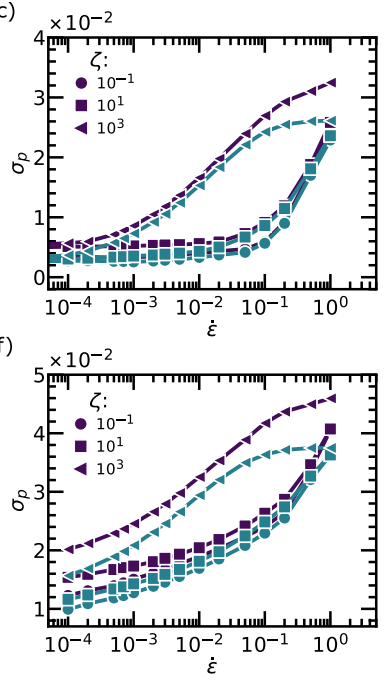

Figure 3.A.3. The influence of driving and friction coefficient on the stress-strain response. (a) Stress versus strain for a network around the isostatic point $(p=0.65, L=1024, \zeta=10$, $T^{*}=1 \times 10^{-5}$ ) for a range of strain rates (see legend). (b) Stress versus strain for a subisostatic network $\left(p=0.65, L=1024, \dot{\varepsilon}=0.001, T^{*}=1 \times 10^{-5}\right.$ ) for a range of $\zeta$ (see legend). (c) $\sigma_{p}$ versus $\dot{\varepsilon}$ for a network with $p=0.65$ and $L=1024$. $\sigma_{p}$ is determined for $T^{*}=1 \times 10^{-9}$ (purple) and $T^{*}=1 \times 10^{-5}$ (blue) the shape of the markers indicates the friction coefficient $\zeta$ (see legend). (d) Stress versus strain for a network far above the isostatic point $(p=0.90, L=1024, \zeta=10$, $T^{*}=1 \times 10^{-5}$ ) for a range of strain rates (see legend). (e) Stress versus strain for a network far above the isostatic point $\left(p=0.90, L=1024, \dot{\varepsilon}=0.001, T^{*}=1 \times 10^{-5}\right)$ for a range of $\zeta$ (see legend). (f) $\sigma_{p}$ versus $\dot{\varepsilon}$ for networks with $p=0.90$ and $L=1024$. Same colour code as in (c).

\section{A.5 Estimate of $E_{\text {therm }} / E_{\text {break }}$ in experimental systems}

We introduce the ratio between the thermal energy $\left(E_{\text {therm }}=k_{\mathrm{B}} T\right)$ and the failure energy of a building block ( $\left.E_{\text {break }}\right)$ as a measure for the (network level) temperature sensitivity of the failure response of a network material. We can use the expression $T^{*} /\left(\frac{1}{2} \lambda^{2}\right)$ to get a rough estimate of $E_{\text {therm }} / E_{\text {break }}$ for an experimental system when we know $\mu$ and $\lambda$ of the individual building blocks. Please note, that this approximation assumes a linear stress-strain response of the building blocks $\left(E_{\text {break }}=\frac{1}{2} \mu\left(\lambda \ell_{0}\right)^{2}\right)$.

\section{Stiff fibre networks: Collagen}

Collagen is a stiff athermal fibre network. The spring constant of a stiff fibre can be defined as $\mu=E_{y} A / \ell_{c}=E_{y} \pi r^{2} / \ell_{c}$ with $E_{y}$ the Young's Modulus of the fibre, $A$ 
the cross-section of the fibre, $\ell_{c}$ the distance between crosslinks, and $r$ the radius of a fibre. Here we neglect the fibre bending stiffness, which is expected to have only a minor influence on network fracture ${ }^{7}$. Consequently, $T^{*}$ of a fibre network can be calculated as

$$
T^{*}=\frac{k_{\mathrm{B}} T}{E_{y} \pi r^{2} \ell_{c}} .
$$

For a collagen network with $E_{y}=50 \mathrm{MPa}, r=30 \mathrm{~nm}$ and assuming that $\ell_{c}$ scales with the mesh size of the network $\ell_{c} \approx \xi=2 \mu \mathrm{m}$ (Based on Ref. [43]) we find that $T^{*}=1.5 \times 10^{-8}$ at $T=298 \mathrm{~K}$, which falls well into the athermal limit. For a value of $\lambda=0.15^{7}$ we find that $T^{*} /\left(\frac{1}{2} \lambda^{2}\right)=1.3 \times 10^{-6}$ which also falls well into the athermal limit.

\section{Semiflexible polymer networks: Actin}

Actin is regarded as a typical example of a semiflexible polymer. For a semiflexible polymer, the spring constant is $\mu=90 \kappa \ell_{p} / \ell^{4}$ (by linear approximation) ${ }^{2}$, with $\ell$ the contour length of the polymer and $\ell_{p}$ its persistence length. Using that $\ell_{p}=\kappa / k_{\mathrm{B}} T$ and $\ell \approx \ell_{c}$ we find that $\mu=90 k_{\mathrm{B}} T \ell_{p}^{2} / \ell_{c}^{4}$, with $\kappa$ the bending stiffness, $\ell_{p}$ the persistence length, and $\ell_{c}$ the distance between crosslinks. Thus, the reduced temperature of a semiflexible polymer, like actin, is

$$
T^{*}=\frac{\ell_{c}^{2}}{90 \ell_{p}^{2}} .
$$

Furthermore, if we assume that a semiflexible polymer breaks once its thermal fluctuations are pulled out, we can calculate the extensibility as the ratio between contour length and the average end-to-end distance without tension. With $\Delta \ell=\ell^{2} /\left(6 \ell_{p}\right)$ the average contraction of a semiflexible polymer with respect to its contour length without tension ${ }^{2}, \lambda=\ell /(\ell-\Delta \ell)-1=\Delta \ell /(l-\Delta \ell)$. Assuming that $\ell \approx \ell_{c}$ and $\Delta \ell \ll \ell_{c}$ we can use the simplified expression $\lambda \approx \ell_{c} /\left(6 \ell_{p}\right)$.

For an actin network with fibres with a persistence length of $17 \mu \mathrm{m}^{2}$ and a distance between crosslinks $\ell_{c}$ of roughly $300 \mathrm{~nm}^{44} T^{*}=3.5 \times 10^{-6}$ which indicates that the effect of temperature at the network level is negligible (note that at the level of the building blocks temperature does play an important role). For these parameters we find that $\lambda=0.03$ and that $T^{*} /\left(\frac{1}{2} \lambda^{2}\right)=8 \times 10^{-3}$, which is in the cross-over regime where the failure response is temperature sensitive. 


\section{Flexible polymer networks: Freely jointed chain}

Flexible polymers are an example of extremely soft network building blocks. In the case of flexible polymers, the elastic response of the building blocks can be described based on the freely jointed chain. By linear approximation, the spring constant of a freely jointed chain can be written as $\mu=3 k_{\mathrm{B}} T /\left(N b^{2}\right)$ with $N$ the number of segments and $b$ the segment length. Thus, we find that

$$
T^{*}=\frac{k_{\mathrm{B}} T}{\mu \ell_{c}^{2}}=\frac{N b^{2}}{3 \ell_{c}^{2}}=\frac{1}{3},
$$

when we use the average end-to-end distance of the chain as an estimate for the distance between crosslinks $l_{c}=\sqrt{N} b$. Furthermore, if we assume that the extensibility of the chain scales as the ratio between the contour length of the polymer $N b$ and the average end-to-end distance $l_{c}$, we find $\lambda=\sqrt{N}-1 \approx \sqrt{N}$. Thus, $T^{*} /\left(\frac{1}{2} \lambda^{2}\right) \approx 2 /(3 N)$. For a polymer network with $N=100$ this results in the estimates $T^{*}=0.33$ and $T^{*} /\left(\frac{1}{2} \lambda^{2}\right)=7 \times 10^{-3}$ i.e. the network level mechanical and failure response are both placed well in the temperature sensitive regime. However, it should be noted that the strong non-linear response of polymer chains might significantly affect this estimate, especially in the failure regime. 
(a)

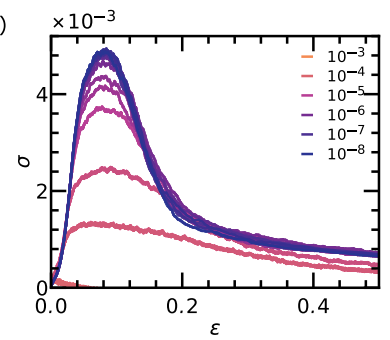

(c) $2.0 \times 10^{-2}$ (b)
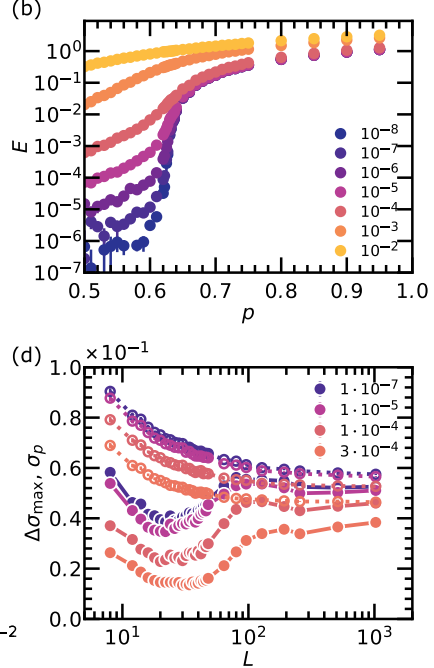

Figure 3.A.4. Main results based on the virial stress including the kinetic component. (a) stressstrain curve for a system with $L=128$ and $p=0.65$ for a range of reduced temperatures $T^{*}$ indicated in the legend. Curves are averages over 10 configurations. (b) linear modulus as a function of the dilution factor $p$ for a range of $T^{*}$ (see legend). (c) Peak stress $\sigma_{p}$ as a function of the reduced temperature for a range of $p$ (see legend). (d) Maximum stress drop $\Delta \sigma_{\text {max }}$ and peak stress $\sigma_{p}$ for networks with $p=0.80$ as a function of system size $L$ for a range of $T^{*}$ (see legend).

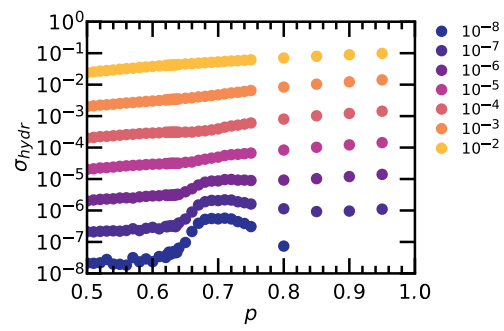

Figure 3.A.5. The time averaged (over 1900 $\tau$ ) hydrostatic stress at $0 \%$ strain as a function of the $p$ for a range of reduced temperatures $T^{*}$ (see legend). Every data point is based on simulations of at least 10 independent configurations. 

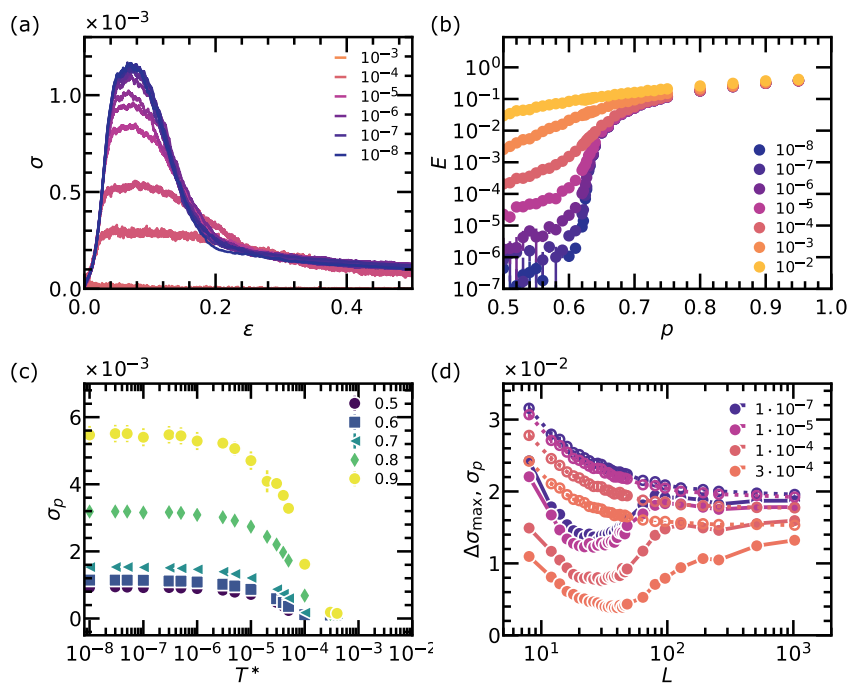

Figure 3.A.6. Main results based on the deviatoric stress $\sigma=\sigma_{y y}-\sigma_{\text {hydr }}$. (a) stress-strain curve for a system with $L=128$ and $p=0.65$ for a range of reduced temperatures $T^{*}$ indicated in the legend. Curves are averages over 10 configurations. (b) linear modulus as a function of the dilution factor $p$ for a range of $T^{*}$ (see legend). (c) Peak stress $\sigma_{p}$ as a function of the reduced temperature for a range of $p$ (see legend). (d) Maximum stress drop $\Delta \sigma_{\max }$ and peak stress $\sigma_{p}$ for networks with $p=0.80$ as a function of system size $L$ for a range of $T^{*}$ (see legend).
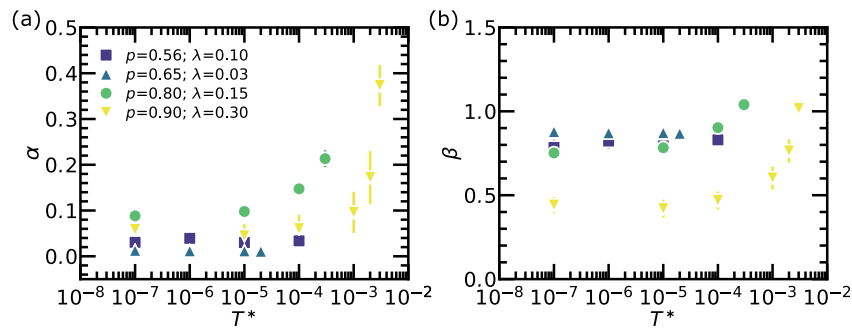

Figure 3.A.7. Fit parameters of the scaling of $\sigma_{p}$ with $L$ using the power law $\sigma_{p}=(L / \alpha)^{-\beta}+\sigma_{p}^{\infty}$. $\sigma_{p}^{\infty}$ is reported in the main text. (a) $\alpha$ versus $T^{*}$. System parameters are indicated in the legend. (b) $\beta$ versus $T^{*}$ for the same systems. Error bars represent the standard error in the fit of $\alpha$ and $\beta$, respectively. 

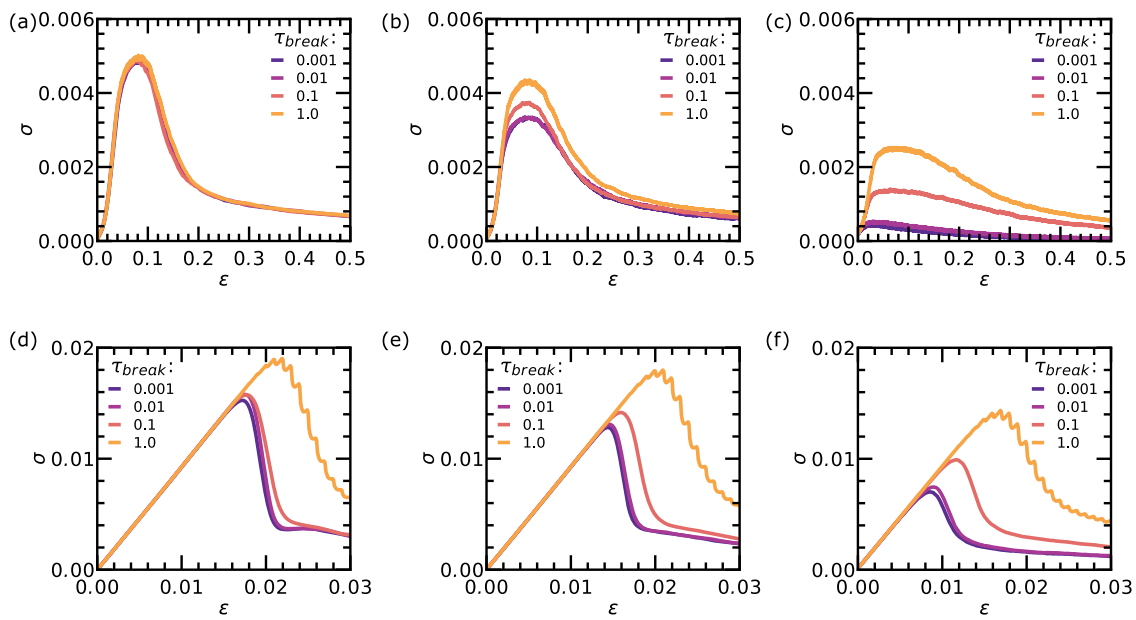

Figure 3.A.8. Effect of bond breaking time $\tau_{\text {break }}$, i.e. the time interval between moments when bonds are allowed to rupture, on the stress-strain response. Stress-strain response for a system with $p=0.65$ and $L=128$ for a temperature of (a) $T^{*}=1 \times 10^{-8}$, (b) $T^{*}=1 \times 10^{-5}$, and (c) $T^{*}=5 \times 10^{-5}$. Stress-strain response for a system with $p=0.90$ and $L=128$ for a temperature of (d) $T^{*}=1 \times 10^{-8}$, (e) $T^{*}=1 \times 10^{-5}$ and (f) $T^{*}=5 \times 10^{-5}$. The curves are averaged over 30 configurations.
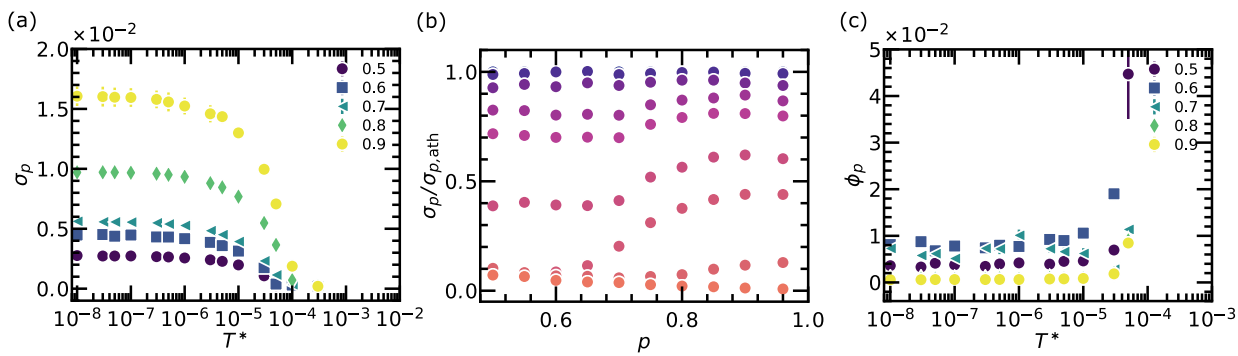

Figure 3.A.9. Main results based on the virial stress $\sigma_{y y}$ for simulations with $\tau_{\text {break }}=0.001 \tau$. Every data point is based on simulations of 10 independent configurations. (a) Peak stress $\sigma_{p}$ as a function of the reduced temperature for a range of $p$ (see legend). (b) Connectivity dependence of $\sigma_{p}$ normalized by the peak stress in the athermal limit $\sigma_{p}$,ath for several reduced temperatures (see legend). (c) Fraction of broken bonds at the peak strain $\phi_{p}$ (including the peak event) as a function of $T^{*}$ for a range of dilution factors $p=0.50-0.90$ (see legend). Fractions are calculated with respect to the initial number of bonds in the diluted network. 


\section{List of symbols}

\begin{tabular}{|c|c|}
\hline Symbol & Description \\
\hline$A$ & Cross sectional area of a fibre \\
\hline$b$ & Segment length or Kuhn length of a polymer chain \\
\hline$d$ & Dimensionality of the system \\
\hline E & Linear modulus, can also be written as $E_{y}$ \\
\hline$E_{\text {therm }}$ & Thermal energy $k_{\mathrm{B}} T$ \\
\hline$E_{\text {frac }}$ & $\begin{array}{l}\text { Elastic energy stored in a bond extended up to the rupture } \\
\text { threshold }\end{array}$ \\
\hline $\mathcal{E}$ & Energy, $\mathcal{E}=1$ in reduced units \\
\hline $\boldsymbol{F}$ & Force vector \\
\hline$k_{\mathrm{B}}$ & Boltzmann constant, $k_{\mathrm{B}}=1$ in reduced units \\
\hline$\ell$ & Bond or spring length (or polymer contour length) \\
\hline$\ell_{0}$ & Rest length of an element or spring \\
\hline$\Delta \ell$ & Extension of a spring with respect to its rest length \\
\hline$\ell_{c}$ & Length of fibre in between crosslinks \\
\hline$\ell_{p}$ & Persistence length \\
\hline$L$ & Lattice size, with $L \times L$ the number of nodes \\
\hline$L_{\min }$ & Network size at which $\Delta \sigma_{\max }$ is minimal (for a given $p, \lambda$ and $T^{*}$ ) \\
\hline$m$ & Mass of a node, $m=1$ in reduced units \\
\hline$N$ & Number of segments in a polymer chain \\
\hline$p$ & $\begin{array}{l}\text { Connectivity parameter, representing the fraction of bonds re- } \\
\text { maining after dilution }\end{array}$ \\
\hline$p_{\text {iso }}$ & Isostatic point defined in terms of $p$ \\
\hline$r$ & Radius of a fibre \\
\hline$r$ & Instantaneous position of a node \\
\hline$r_{\text {init }}$ & Initial positions of nodes prior to equilibration \\
\hline$\overline{\boldsymbol{r}}$ & Time averaged position of a node \\
\hline$\overline{\boldsymbol{r}}_{0}$ & Time averaged position of a node at $0 \%$ strain \\
\hline$\overline{\boldsymbol{r}}_{\text {aff }}$ & $\begin{array}{l}\text { Position of a node assuming affine displacement with respect to } \\
\text { the time averaged position of individual nodes at rest }\end{array}$ \\
\hline$d r$ & $\begin{array}{l}\text { Average distance between the node position prior to equilibration } \\
\boldsymbol{r}_{\text {init }} \text { and the average node position after equilibration } \overline{\boldsymbol{r}}_{0}\end{array}$ \\
\hline$d r_{\text {fluc }}$ & $\begin{array}{l}\text { Root mean square size of thermal fluctuations with respect to the } \\
\text { equilibrium position of a node }\end{array}$ \\
\hline
\end{tabular}




\begin{tabular}{|c|c|}
\hline$R(t)$ & White noise with zero-mean \\
\hline$t$ & Time \\
\hline$\tau$ & Unit of time, $\tau=\sqrt{m \ell_{0}^{2} / \mathcal{E}}$ \\
\hline$\tau_{0}$ & Relaxation time \\
\hline$T$ & System temperature \\
\hline$T^{*}$ & Reduced temperature \\
\hline$u_{\text {aff }}$ & $\begin{array}{l}\text { Displacement vector of node position after affine deformation } \\
\text { with respect to the initial average node position } \overline{\boldsymbol{r}}_{0}\end{array}$ \\
\hline$u_{\text {naff }}$ & $\begin{array}{l}\text { Displacement vector from affine node position due to (time- } \\
\text { averaged) non-affine network rearrangements }\end{array}$ \\
\hline$u_{\text {therm }}$ & $\begin{array}{l}\text { (Instantaneous) displacement vector from average particle position } \\
\text { due to thermal fluctuations }\end{array}$ \\
\hline$z$ & Connectivity, number of bonds connected to a node \\
\hline$\langle z\rangle$ & Average connectivity over all nodes \\
\hline$z_{\max }$ & Maximum connectivity \\
\hline$\Gamma$ & Non-affinity parameter based on instantaneous node positions \\
\hline$\Gamma_{\text {mech }}$ & $\begin{array}{l}\text { Non-affinity parameter based on time-averaged node positions } \\
\text { measured during a strain-step protocol }\end{array}$ \\
\hline$\epsilon$ & Uniaxial strain \\
\hline$\dot{\epsilon}$ & Strain rate \\
\hline$\epsilon_{p}$ & Peak strain, strain corresponding to $\sigma_{p}$ \\
\hline$\zeta$ & Friction coefficient \\
\hline$\mu$ & Spring constant, such that $F=\mu \Delta \ell$ \\
\hline$\xi$ & Mesh size \\
\hline$\kappa_{e}$ & Excess kurtosis \\
\hline$\kappa$ & Bending rigidity of a fibre \\
\hline$\lambda$ & Breaking threshold of a spring defined as $\lambda=\left(l_{\text {break }}-l_{0}\right) / l_{0}$ \\
\hline$\sigma$ & Stress \\
\hline$\sigma_{p}$ & $\begin{array}{l}\text { Peak stress or strength, maximum stress that can be carried by the } \\
\text { network }\end{array}$ \\
\hline$\sigma_{p}^{\infty}$ & Peak stress in the thermodynamic limit \\
\hline$\sigma_{\text {ath }}$ & Stress response in the athermal limit \\
\hline$\sigma_{\mathrm{p}, \text { ath }}$ & Peak stress in the athermal limit \\
\hline$\sigma_{\mathrm{p}, \mathrm{ath}}{ }^{\infty}$ & Peak stress in the athermal limit and the thermodynamic limit \\
\hline$\Delta \sigma_{\max }$ & Abruptness or maximum drop in stress after $\sigma_{p}$ \\
\hline
\end{tabular}


$\phi$

$\phi_{p}$

$\phi_{f}$
Fraction of broken bonds with respect to the number of intact bonds in the network prior to deformation

Fraction of broken bonds at the peak strain including the peak event

Fraction of broken bonds after complete network rupture

\section{References}

[1] R. C. Picu, Mechanics of random fiber networks - A review. Soft Matter 7, 6768-6785 (2011).

[2] C. P. Broedersz, F. C. Mackintosh, Modeling semiflexible polymer networks. Reviews of Modern Physics 86, 995-1036 (2014).

[3] K. A. Jansen, A. J. Licup, A. Sharma, R. Rens, F. C. MacKintosh, G. H. Koenderink, The Role of Network Architecture in Collagen Mechanics. Biophysical Journal 114, 2665-2678 (2018).

[4] P. H. Kouwer, M. Koepf, V. A. Le Sage, M. Jaspers, A. M. Van Buul, Z. H. EksteenAkeroyd, T. Woltinge, E. Schwartz, H. J. Kitto, R. Hoogenboom, S. J. Picken, R. J. Nolte, E. Mendes, A. E. Rowan, Responsive biomimetic networks from polyisocyanopeptide hydrogels. Nature 493, 651-655 (2013).

[5] C. Creton, 50th Anniversary Perspective: Networks and Gels: Soft but Dynamic and Tough. Macromolecules 50, 8297-8316 (2017).

[6] M. Bouzid, E. Del Gado, Network Topology in Soft Gels: Hardening and Softening Materials. Langmuir 34, 773-781 (2018).

[7] F. Burla, S. Dussi, C. Martinez-Torres, J. Tauber, J. van der Gucht, G. H. Koenderink, Connectivity and plasticity determine collagen network fracture. Proceedings of the National Academy of Sciences of the United States of America 117, 8326-8334 (2020).

[8] C. Creton, M. Ciccotti, Fracture and adhesion of soft materials: A review. Reports on Progress in Physics 79, 46601 (2016).

[9] F. J. Vernerey, R. Brighenti, R. Long, T. Shen, Statistical Damage Mechanics of Polymer Networks. Macromolecules 51, 6609-6622 (2018).

[10] C. P. Broedersz, X. Mao, T. C. Lubensky, F. C. Mackintosh, Criticality and isostaticity in fibre networks. Nature Physics 7, 983-988 (2011).

[11] A. Sharma, A. J. Licup, K. A. Jansen, R. Rens, M. Sheinman, G. H. Koenderink, F. C. Mackintosh, Strain-controlled criticality governs the nonlinear mechanics of fibre networks. Nature Physics 12, 584-587 (2016).

[12] L. Zhang, D. Z. Rocklin, L. M. Sander, X. Mao, Fiber networks below the isostatic point: Fracture without stress concentration. Physical Review Materials 1, 052602 (2017). 
[13] S. Dussi, J. Tauber, J. Van Der Gucht, Athermal Fracture of Elastic Networks: How Rigidity Challenges the Unavoidable Size-Induced Brittleness. Physical Review Letters 124, 18002 (2020).

[14] M. M. Driscoll, B. G. G. Chen, T. H. Beuman, S. Ulrich, S. R. Nagel, V. Vitelli, The role of rigidity in controlling material failure. Proceedings of the National Academy of Sciences of the United States of America 113, 10813-10817 (2016).

[15] E. Berthier, J. E. Kollmer, S. E. Henkes, K. Liu, J. M. Schwarz, K. E. Daniels, Rigidity percolation control of the brittle-ductile transition in disordered networks. Physical Review Materials 3, 075602 (2019).

[16] J. C. Maxwell, On reciprocal figures and diagrams of forces. The London, Edinburgh, and Dublin Philosophical Magazine and Journal of Science 27, 250-261 (1864).

[17] M. Wyart, H. Liang, A. Kabla, L. Mahadevan, Elasticity of floppy and stiff random networks. Physical Review Letters 101, 215501 (2008).

[18] J. L. Shivers, S. Arzash, A. Sharma, F. C. MacKintosh, Scaling Theory for Mechanical Critical Behavior in Fiber Networks. Physical Review Letters 122, 188003 (2019).

[19] J. Feng, H. Levine, X. Mao, L. M. Sander, Nonlinear elasticity of disordered fiber networks. Soft Matter 12, 1419-1424 (2016).

[20] A. Kulachenko, T. Uesaka, Direct simulations of fiber network deformation and failure. Mechanics of Materials 51, 1-14 (2012).

[21] S. Borodulina, H. R. Motamedian, A. Kulachenko, Effect of fiber and bond strength variations on the tensile stiffness and strength of fiber networks. International Journal of Solids and Structures 154, 19-32 (2018).

[22] S. Deogekar, R. C. Picu, On the strength of random fiber networks. Journal of the Mechanics and Physics of Solids 116, 1-16 (2018).

[23] S. Deogekar, M. R. Islam, R. C. Picu, Parameters controlling the strength of stochastic fibrous materials. International Journal of Solids and Structures 168, 194-202 (2019).

[24] H. M. James, E. Guth, Theory of the elastic properties of rubber. The Journal of Chemical Physics 11, 455-481 (1943).

[25] E. M. Arruda, M. C. Boyce, A three-dimensional constitutive model for the large stretch behavior of rubber elastic materials. Journal of the Mechanics and Physics of Solids 41, 389-412 (1993).

[26] M. Dennison, M. Sheinman, C. Storm, F. C. Mackintosh, Fluctuation-stabilized marginal networks and anomalous entropic elasticity. Physical Review Letters 111, 095503 (2013).

[27] L. Zhang, X. Mao, Finite-temperature mechanical instability in disordered lattices. Physical Review E 93, 022110 (2016).

[28] G. I. Bell, Models for the specific adhesion of cells to cells. Science 200, 618-627 (1978).

[29] J. T. Bullerjahn, S. Sturm, K. Kroy, Theory of rapid force spectroscopy. Nature Communications 5, 4463 (2014). 
[30] P. J. Skrzeszewska, J. Sprakel, F. A. de Wolf, R. Fokkink, M. A. Cohen Stuart, J. van der Gucht, Fracture and Self-Healing in a Well-Defined Self-Assembled Polymer Network. Macromolecules 43, 3542-3548 (2010).

[31] S. Plimpton, Fast parallel algorithms for short-range molecular dynamics. Journal of Computational Physics 117, 1-19 (1995).

[32] S. Bonfanti, E. E. Ferrero, A. L. Sellerio, R. Guerra, S. Zapperi, Damage Accumulation in Silica Glass Nanofibers. Nano Letters 18, 4100-4106 (2018).

[33] P. R. Onck, T. Koeman, T. Van Dillen, E. Van Der Giessen, Alternative explanation of stiffening in cross-linked semiflexible networks. Physical Review Letters 95, 178102 (2005).

[34] A. Sharma, A. J. Licup, R. Rens, M. Vahabi, K. A. Jansen, G. H. Koenderink, F. C. MacKintosh, Strain-driven criticality underlies nonlinear mechanics of fibrous networks. Physical Review E 94, 042407 (2016).

[35] G. Žagar, P. R. Onck, E. Van Der Giessen, Two fundamental mechanisms govern the stiffening of cross-linked networks. Biophysical Journal 108, 1470-1479 (2015).

[36] A. S. G. Van Oosten, M. Vahabi, A. J. Licup, A. Sharma, P. A. Galie, F. C. MacKintosh, P. A. Janmey, Uncoupling shear and uniaxial elastic moduli of semiflexible biopolymer networks: compression-softening and stretch-stiffening. Scientific Reports 6, 19270 (2016).

[37] G. A. Buxton, N. Clarke, Bending to stretching transition in disordered networks. Physical Review Letters 98, 238103 (2007).

[38] M. J. Alava, P. K. V. V. Nukala, S. Zapperi, Statistical models of fracture. Advances in Physics 55, 349-476 (2006).

[39] C. Heussinger, E. Frey, Force distributions and force chains in random stiff fiber networks. European Physical Journal E 24, 47-53 (2007).

[40] R. C. Arevalo, P. Kumar, J. S. Urbach, D. L. Blair, Stress heterogeneities in sheared type-I collagen networks revealed by boundary stress microscopy. PLOS ONE 10, 0118021 (2015).

[41] L. Liang, C. Jones, S. Chen, B. Sun, Y. Jiao, Heterogeneous force network in 3D cellularized collagen networks. Physical Biology 13, 066001 (2016).

[42] H. Laubie, F. Radjai, R. Pellenq, F. J. Ulm, Stress Transmission and Failure in Disordered Porous Media. Physical Review Letters 119, 075501 (2017).

[43] A. M. Stein, D. A. Vader, D. A. Weitz, L. M. Sander, The micromechanics of threedimensional collagen-I gels. Complexity 16, 22-28 (2011).

[44] M. L. Gardel, Elastic Behavior of Cross-Linked and Bundled Actin Networks. Science 1301, 1301-1305 (2009). 



\section{PART II}

\section{Elasticity and fracture of composite networks}





\section{Chapter 4}

\section{Stress management in composite biopolymer networks}

Living tissues show an extraordinary adaptiveness to strain, which is crucial for their proper biological functioning. The physical origin of this mechanical behaviour has been widely investigated using reconstituted networks of collagen fibres, the principal load-bearing component of tissues. However, collagen fibres in tissues are embedded in a soft hydrated polysaccharide matrix which generates substantial internal stresses whose effect on tissue mechanics is unknown. We combine rheology experiments and simulations to study the mechanical response of a biomimetic model system composed of the two paradigmatic tissue components: collagen and hyaluronan. We observe a synergistic mechanics characterized by an enhanced stiffness in the linear regime and a delayed strain-stiffening, strongly dependent on hyaluronan concentration. We demonstrate that internal stresses and elastic reinforcement, both generated by the matrix, together with the fibre rigidity are independent handles to tune the composite response, unveiling therefore new principles for material design.

Federica Burla*, Justin Tauber*, Simone Dussi, Jasper van der Gucht \& Gijsje H. Koenderink

"Stress management in composite biopolymer networks"

Nature Physics 15, 549-553 (2019)

*These authors contributed equally to this work 


\subsection{Introduction}

The soft tissues in our body such as the skin, muscles and arteries have a striking ability to switch from being soft at small deformations to being stiff at high deformations ${ }^{1-3}$. This adaptive response to strain enables tissues to accommodate dynamic processes such as cell proliferation and migration ${ }^{4}$, while preventing tissue rupture ${ }^{5}$. Recent biophysical studies have revealed that the main determinant of tissue strain-stiffening is collagen ${ }^{6-8}$, a scaffolding protein that forms fibrillar networks ${ }^{9}$. Collagen networks have the intrinsic ability to undergo a transition from soft to rigid when strained ${ }^{10}$ because at the coarse-grained level of fibres they have a sub-isostatic architecture, meaning that the average number of fibres meeting at each junction ( 3 for branches and 4 for crosslinked fibres) is below the Maxwell stability criterion of 6 for a network of springs ${ }^{11}$. Fibrous networks are soft at small strains because they respond primarily by fibre bending, but stiffen at large deformations as the fibres align along the principal direction of strain and start to stretch ${ }^{7,8,12}$. However, collagen networks within tissues are always embedded in a soft hydrated matrix comprised of polysaccharides and glycosylated proteins whose mechanical role is unknown ${ }^{13}$. Recent computational models suggest that these matrix polymers might elastically reinforce the fibrillar collagen matrix ${ }^{14,15}$. However, the role of the matrix is likely more complex, because the constituent molecules carry large negative charges ${ }^{16}$. It has been shown that the matrix consequently generates substantial mechanical stresses, especially in cartilaginous tissues ${ }^{17-19}$, which could potentially have a large impact on tissue mechanics given the strong strain-sensitivity of collagen.

Here, by combining mechanical measurements and computer simulations, we show that networks composed of collagen fibres and a hyaluronan matrix exhibit synergistic mechanics characterized by an enhanced stiffness and delayed strainstiffening. We demonstrate that the polysaccharide matrix has a dual effect on the composite response involving both internal stress and elastic reinforcement. Our findings elucidate how tissues can tune their strain-sensitivity over a wide range and provide a novel design principle for synthetic materials with programmable mechanical properties.

\subsection{Model and methods}

\subsubsection{Experimental methods}

Here we briefly introduce the relevant experimental methods and refer to the original paper ${ }^{20}$ for further details. 


\section{Sample preparation}

The tissue-mimetic composite system was prepared by mixing bovine dermal collagen type I atelocollagen or telocollagen and thiol-modified hyaluronan. The networks were co-assembled in phosphate buffered saline with the $\mathrm{pH}$ set to pH 7.4 using $0.1 \mathrm{~m} \mathrm{NaOH}$. Finally, the PEGDA (polyethylene glycol diacrylate) crosslinker $(3.4 \mathrm{kDa})$ in a concentration ratio of $1: 4$ with hyaluronan (molar ratio 20:1) was added just before inserting the sample in the rheometer or microscopy sample cells, to prevent premature crosslinking of the hyaluronan networks. The experiments were performed at a fixed concentration of $1 \mathrm{mg} \mathrm{ml}^{-1}$ collagen and at hyaluronan concentrations ranging from 0 to $7 \mathrm{mg} \mathrm{ml}^{-1}$.

\section{Rheology measurements}

All the experiments were performed on an Anton Paar Physica MCR501 rheometer, equipped with a cone plate geometry having a diameter of $40 \mathrm{~mm}$ and cone angle of $1^{\circ}$. We verified that the measurements were independent of gap size by repeating the experiments with a parallel plate geometry at gaps of 200 and $400 \mu \mathrm{m}$ (data not shown). The samples were allowed to polymerize for two hours at a temperature of $37^{\circ} \mathrm{C}$, maintained by a Peltier plate, while monitoring the evolution of the linear shear moduli with a small amplitude oscillatory strain $(0.5 \%$ strain, $0.5 \mathrm{~Hz})$. The steady-state values of the linear viscoelastic moduli were calculated as an average over the last ten data points of the polymerization curve, and the average reported is representative of at least 3 independent measurements. After polymerization, the non-linear elastic response was measured using a well-established prestress protocol $^{21}$. Briefly, a constant stress $\sigma$ was applied for $30 \mathrm{~s}$, to probe for network creep, and then an oscillatory stress $\delta \sigma$ was superposed with an amplitude of $1 / 10$ of $\sigma$. The resulting differential strain $\delta \gamma$ was then used to calculate the differential (or tangent) modulus $K^{\prime}=\delta \sigma / \delta \gamma$.

\section{Determination of linear mechanical enhancement, onset and critical strain}

The linear mechanical enhancement was determined by dividing the elastic modulus $G_{0}$ measured at the end of polymerization of the composite network $\left(G_{\text {meas }}\right)$ by the sum $\left(G_{\text {sum }}\right)$ of the elastic moduli $G_{0}$ of the two individual components. The error bar shown is calculated from error propagation. The rheology data in the non-linear regime were evaluated using a custom-written Python routine. Consistent with a definition that was previously introduced in the context of fibrillar network mechanics ${ }^{22}$, for the onset strain determination we first considered the curve $K^{\prime} / \sigma$ vs $\sigma$. Subsequently, we performed a cubic spline interpolation and 
determined the minimum of the resulting curve. The associated stress was characterized as the onset stress $\sigma_{0}$, and the corresponding strain was taken to be the onset strain $\gamma_{0}$. To determine the critical strain, we calculated the cubic spline derivative of the curve $\log K^{\prime}$ as a function of $\log \gamma$. The strain at which this function attained its maximum was taken to be the critical strain $\gamma_{c}$. All the characteristic strain values are shown as average with standard error of the mean of at least three measurements.

\section{Determination of collagen bending rigidity}

To determine the bending rigidity of collagen fibres prepared from either atelocollagen or telocollagen, we measured the non-linear elastic response of pure collagen networks prepared at $1 \mathrm{mg} \mathrm{ml}^{-1}$ using the prestress protocol described above. The dimensionless bending rigidity, was extracted from the stress-stiffening curves by comparing them to curves obtained with the following analytical expression valid for submarginal fibrous networks described by Sharma et al. ${ }^{8}$ :

$$
\frac{\tilde{\kappa}}{|\Delta \gamma|^{\phi}} \approx \frac{K^{\prime}}{|\Delta \gamma|^{f}}\left( \pm 1+\frac{K^{\prime \frac{1}{f}}}{|\Delta \gamma|}\right)^{(\phi-f)}
$$

where $\tilde{\kappa}$ is the free parameter corresponding to the dimensionless bending rigidity, $K^{\prime}$ is the measured value of differential modulus, $|\Delta \gamma|$ the distance between the considered $\gamma$ and the critical strain, and $f$ and $\phi$ critical exponents set by the network architecture, here taken to be 0.77 and 2.2 by comparing the measured values of onset and critical strain with published results ${ }^{22}$.

\subsubsection{Computational methods}

\section{Composite network simulations}

We model the tissue-like composite networks as an athermal and submarginal fibre network that represents the collagen network, embedded in a homogeneous (not diluted) matrix of linear elastic springs that represents the linearly elastic hyaluronan matrix ${ }^{14,23}$. All simulations are performed on a lattice of $L$ by $L$ nodes, where $L=50$, with initial lattice spacing $d=1$. For selected cases, we perform simulations with $L=100$ to confirm that size effects are within the statistical errors presented in the main text. The fibre network is modelled on a triangular lattice, using a dilution procedure to achieve a maximum local connectivity $z$ of 4 bonds per node. As shown previously with similar models ${ }^{7,22}$, it is possible to quantitatively describe the non-linear elastic properties of reconstituted collagen 
networks, provided that model-dependent parameters, such as connectivity and number of crosslinks per fibre, are properly matched ${ }^{7,22,24}$. For these simulations we consider networks with an average connectivity $\langle z\rangle=3.4$. The total energy of the system is:

$$
\begin{aligned}
E & =E_{\text {stretch }}^{\text {fibre }}+E_{\text {bend }}^{\text {fibre }}+E_{\text {stretch }}^{\text {matrix }} \\
& =\sum_{\langle i j\rangle} \frac{1}{2} \frac{\mu_{1}}{\ell_{0,1}}\left(\ell-\ell_{0,1}\right)^{2}+\sum_{\langle i j k\rangle} \frac{1}{2} \frac{\kappa_{1}}{\ell_{0,1}}\left(\theta-\theta_{0,1}\right)^{2}+\sum_{\langle i j\rangle} \frac{1}{2} \frac{\mu_{2}}{\ell_{0,2}}\left(\ell-\ell_{0,2}\right)^{2}
\end{aligned}
$$

where $\mu_{1}$ and $\kappa_{1}$ are the stretching and bending constant for the fibre segments, respectively, and $\mu_{2}$ is the stretching constant for the matrix segments. Nearestneighbour nodes are indicated with indices $i j$, and $\ell$ is the distance between the two nodes (spring length). Bending contributions are associated only to segments belonging to the same straight fibre, that are indicated with the triplet $i j k, \theta$ denotes the angle between the triplet and $\theta_{0,1}$ the rest angle. The rest lengths $\ell_{0,1}$ and $\ell_{0,2}$ of the fibre and matrix segments are expressed in terms of the initial lattice spacing $d$ (we use $\ell_{0,1}=d$ in all simulations), and we use the reduced quantities $\tilde{\mu}_{2}=\mu_{2} / \mu_{1}$ and $\tilde{\kappa}=\kappa /\left(d^{2} \mu_{1}\right)$. We vary the matrix properties $\tilde{\mu}_{2}$ from $1 \times 10^{-5}$ to $1 \times 10^{-3}$ (Figure 4.A.4) while the fibre bending rigidity $\tilde{\kappa}$ is either $1 \times 10^{-4}$ or $3 \times 10^{-5}$, corresponding to the measured values for atelocollagen and telocollagen networks. The mechanical behaviour of the composite networks is obtained in three steps (see Section 4.A.1 for a detailed description). First, to simulate the compressive stress exerted by the hyaluronan matrix, we reduce $\ell_{0,2}$, which we vary from 0.50 to 1.00 . Secondly, we allow the system to achieve an internal balance between the fibre network and the matrix via (isotropic) bulk compression in steps of $0.1 \%$ strain, with each step followed by energy minimization, until a minimum in the total energy as a function of bulk strain is reached. Thirdly, we perform a quasistatic shear simulation in steps of $0.1 \%$ strain, using Lees-Edwards boundary conditions in the $y$-direction and standard periodic boundary conditions in the $x$-direction. The energy minimization is performed using the FIRE algorithm ${ }^{25}$. The stress is obtained as $\sigma=\frac{1}{A} \frac{d E}{d \gamma}$ and the elastic shear modulus as $K=\frac{1}{A} \frac{d^{2} E}{d \gamma^{2}}$, where $A$ is the area of the system. We indicate the linear modulus (calculated for strains $\gamma<2 \%$ ) with $G_{0}$. We obtain the onset strain $\gamma_{o}$ and critical strain $\gamma_{c}$ that characterize the strain-stiffening response from the dependence of $\frac{K}{\sigma}$ on $\sigma$. The first minimum corresponds to $\left(\gamma_{o}, \sigma_{o}\right)$, where $\sigma_{o}$ is the onset stress where stiffening sets in. The subsequent maximum corresponds to $\left(\gamma_{c}, \sigma_{c}\right)$, where $\sigma_{c}$ is the critical stress. 


\section{Model assumptions}

Several computational models have been proposed to describe collagen mechanics at different length scales ${ }^{10}$. We here adapt a spring network model based on the coarse-graining paradigm in which collagen fibres are treated as an athermal elastic material with a given Young's modulus and bending stiffness. Fibres are subsequently discretized in linearly elastic springs to which a stretching constant $\mu$ and bending constant $\kappa$ are associated. This minimal model has already been proven to correctly capture the physics behind the strain-stiffening of collagen networks. ${ }^{8}$ Importantly, collagen fibre networks are sub-isostatic and, therefore, bending-dominated at small strains, so the strain-stiffening behaviour corresponds to the microscopic bending-to-stretching transition of the fibres. Our model could in principle be extended to capture sequential straightening and loading by modelling the fibres as springs with a non-linear stretching response. However, this non-linear response would be relevant only significantly beyond the critical strain at which the network mechanical response is stretching-dominated. To describe the hyaluronan network mechanics, we employ a uniform mesh and do not include bending contribution in the hyaluronan springs. This is justified by the fact that (i) hyaluronan is known to be a quite flexible polymer with a persistence length of only $5-10 \mathrm{~nm}$; (ii) due to the large mismatch in network mesh-sizes, the much finer hyaluronan network appears as a homogenous matrix at the lengthscales relevant for the mechanical response of collagen fibres; (iii) the experimental modulus versus strain curves of the pure hyaluronan network indicate that the hyaluronan network effectively behaves like a linearly elastic matrix in the range of strain interesting for the collagen and composite mechanics. Details on the network geometries used in this work are given below. Examples of how the mesh geometry affects the strain-stiffening of sub-isostatic networks can be found in Refs. [26-29], where it is shown that this does not play a significant role. We use a lattice model because this is computationally much more efficient compared to e.g. Mikado models, especially in the case of two superimposed networks. Furthermore, the use of a triangular mesh rather than square or hexagonal allows us to tune the connectivity of the fibre network to physiological values between 3 and 4 , which is not possible with a hexagonal lattice. The use of a square lattice also represents a limitation to study connectivities close to 4 , since many system-spanning fibres would be present in the system giving rise to an unphysical mechanical response. Prior work on single-component systems showed that lattice models provide predictions that are fully consistent with 2D Mikado networks and 3D networks. 8,26 Physically, this can be understood by the fact that the network mechanics is controlled only by a few parameters, such as the average connectivity 
$\langle z\rangle$ and the average number of crosslinks per fibre $L_{0} / \ell_{c}$, with $L_{0}$ the average fibre length and $\ell_{c}$ the average distance between crosslinks on a fibre. Therefore, mechanically equivalent networks can be constructed using either a regular lattice or a disordered network.

\section{Simulations of an effective single-component network}

To achieve a quantitative comparison with the experimental system, we develop an effective single-component model. In this case, we use a phantom fibre network with an average connectivity $\langle z\rangle=3.2$, which for this network topology matches more closely the mechanical properties of the bare collagen system ${ }^{8,22}$. The presence of the hyaluronan matrix is implicitly implemented as follows. The effect of the matrix stiffness is captured by an increase of the fibre bending stiffness $\tilde{\kappa}$ to $\tilde{\kappa}_{\text {eff }}$, similar to recent simulations of the linear elastic properties of two-component composites $^{14}$. To account for the local compression of the collagen network by the hyaluronan matrix, we isotropically compress the system in steps of $0.1 \%$ strain, followed by energy minimization. Subsequently, all the rest angles $\theta_{0}$ of the fibres are re-set to their value after compression to ensure mechanical equilibrium, meaning that the system retains its shape even if the boundaries are removed. To obtain the network mechanical response, a quasistatic shear simulation is performed in the same manner as described above.

\section{Constructing an effective single-component model}

Recent simulations ${ }^{14}$ have shown that in the linear elastic regime, the effect of an elastic background network on the mechanics of a fibrous network can be mapped onto an effective single-component model. The basic idea is that the reinforcing effect of the matrix can be accounted for by a modified fibre bending rigidity, $\tilde{\kappa}_{\text {eff }}=\tilde{\kappa}+\tilde{\mu}_{2}$ for a triangular network, where $d$ is the lattice spacing. In this work, we tested this model also in the non-linear regime on a phantom network geometry. As shown in Figure 4.A.10, we find that an increase in $\tilde{\mathcal{K}}_{\text {eff }}$ only affects the linear regime. To implement the second effect of a hyaluronan matrix on the collagen network, which is to exert a compressive stress, we therefore need to extend the effective single-component model. In the two-component model, the fibre network was compressed by the matrix as a result of the mechanical equilibrium obtained via energy minimization. In an effective single-component system, we can still perform a bulk compression to simulate the compressive effect of the matrix. This is done in steps of $0.1 \%$ bulk strain. Similar to the two-component simulations, we again observe bending of the fibres, as can be seen in Figure 4.A.10. Furthermore, the introduction of compressive stress alone is able to capture the delay of non-linear 
stiffening that we observe experimentally in collagen/hyaluronan composites. We note that this effect of internal stress resembles the effect of external compression in fibre networks reported in literature ${ }^{30}$. However, our procedure does not yet correctly reproduce the linear regime behaviour. In fact, when we apply bulk compression to the whole system, fibres bend to account for the smaller system size, which results in a positive contribution to the energy. After the bulk compression we switch to a shear deformation. Because the system reorients along the shear direction, some of the bent fibres can straighten out again, leading to a lower system energy upon increasing the shear strain. This implies a negative shear stress and also a negative modulus. At higher shear strains, other fibres are forced to bend and the total energy increases again. The mechanical balance between the fibre network and the background matrix is, therefore, not yet correctly captured in this single-component model. To achieve an internal force balance, we reset all the fibre rest angles to the angles obtained after compression. In other words, the new rest state of the fibres of the single-component model is their bent configuration (Figure 4.A.9). As bending is the only significant energy contribution after compression, this brings the energy of the system close to zero again. This procedure removes the negative shear stresses in the linear regime and still leads to a delay of stiffening in the non-linear regime (Figure 4.A.10).

\section{Mapping the experimental data on the effective single-component model}

The double network model reproduces the experimentally observed delay in stiffening of collagen networks upon the addition of a hyaluronan matrix, but only qualitatively. This is a consequence of a mismatch in geometry between the experimental system and the model. In particular, it can be explained by considering the average number of crosslinks per fibre, calculated as $L_{0} / \ell_{c}$, with $L_{0}$ the average fibre length and $\ell_{c}$ the average distance between crosslinks on a fibre. For the diluted triangular network, for connectivities between 3 and 4 , we have $L_{0} / \ell_{c} \approx 2$, which is much lower than for geometries that have been used successfully in quantitative mapping of collagen elasticity ${ }^{22,31}$. As an example, Mikado networks have $L_{0} / \ell_{c}=11$ at a connectivity of 3.613 and a triangular phantom lattice can have values of $L_{0} / \ell_{c}$ ranging from 2.59 to 10.99 for a connectivity between 3 and $4 .{ }^{22}$ Unfortunately, the phantom lattice geometry is not convenient for explicit doublenetwork simulations, since the phantomization procedure would lead to nodes with 12 matrix bonds and further assumptions on the springs connecting the phantom nodes would be necessary. Therefore, we performed double network simulations on diluted triangular network but effective single-component network simulations on phantom lattices. We performed single-component network simulations for 
lattices with an average connectivity $\langle z\rangle=3.2$, chosen because the critical and onset strain at $0 \%$ compression $\left(\gamma_{o}=0.068 \pm 0.007, \gamma_{c}=0.13 \pm 0.02\right)$ are closer to the strains measured for the collagen-hyaluronan composite $\left(c_{\mathrm{HA}}=2 \mathrm{mg} \mathrm{ml}^{-1}, \gamma_{o}=\right.$ $0.073 \pm 0.006, \gamma_{c}=0.15 \pm 0.01$ ) compared to the results of our simulations obtained with $\langle z\rangle=3.4\left(\gamma_{o}=0.05, \gamma_{c}=0.09\right)$. Subsequently, we constructed maps of $G_{0}$, $\gamma_{o}$ and $\gamma_{c}$ as a function of bulk compression (26 points ranging from $0 \%$ to $25 \%$ ) and effective bending rigidity (51 points ranging from $\tilde{\kappa}_{\text {eff }}=1 \times 10^{-5} \ldots 5 \times 10^{-3}$. We then mapped experimentally determined values of $G_{0}, \gamma_{0}$ and $\gamma_{c}$ obtained for composite networks with different HA concentrations onto the simulation results (Figure 4.A.10). The goal of this quantitative mapping procedure is to find the relation between $c_{\mathrm{HA}}$ (the control parameter of the experiments), the values for the effective fibre bending rigidity and the level of bulk compression (the control parameters of the effective single component model). To achieve this goal, we need to match the output from the experiments $\left(G_{0}, \gamma_{0}\right.$ and $\left.\gamma_{c}\right)$ with the output from the simulations $\left(G_{0} d / \mu_{1}, \gamma_{0}\right.$ and $\left.\gamma_{c}\right)$ for every experimental measurement.

To allow quantitative mapping, the experimental moduli expressed in units of Pascal had to be converted to the dimensionless quantities used in the simulations. First, the linear elastic moduli of composite networks were normalized with respect to the modulus found at $c_{\mathrm{HA}}=0 \mathrm{mg} \mathrm{ml}^{-1}$. Subsequently, the normalized moduli were multiplied by the linear modulus obtained in the effective single component simulations of this work (Figure 4.4(c)) with $\tilde{\kappa}_{\text {eff }}$ set to the $\tilde{\kappa}$ values found by mapping measured strain-stiffening curves for the atelocollagen and telocollagen networks on simulation results ${ }^{22}$ as described in the Methods section $\left(\tilde{\kappa}=1 \times 10^{-5}\right.$ and $1 \times 10^{-4}$, for the atelocollagen and telocollagen, respectively). The set of matching simulation parameters is determined in the following way: we start with the two-dimensional simulation phase space with $\tilde{\kappa}_{\text {eff }}$ on one axis and the level of bulk compression on the other axis. In this phase space we obtained the linear modulus, the onset strain and the critical strain for 1326 simulation points using the effective single component model. These points form a map for every single output parameter as a function of $\tilde{\kappa}_{\text {eff }}$ and the bulk compression. For every experimental measurement (i.e. every hyaluronan concentration), we want to find the point in this simulation phase space that best matches all the experimental output parameters. Because the dependence of the simulation output parameters on the control parameters is smooth, we can draw contour lines that separate the region in phase space with simulation output parameters that are higher than those found in experiment from the region where simulation output parameters are lower. Figure 4.A.11 shows an example of how this is done in case of composites of atelocollagen and $5 \mathrm{mg} \mathrm{ml}^{-1}$ hyaluronan. For most values of $c_{\mathrm{HA}}$, the curves corresponding to the experimental $G_{0}$ and $\gamma_{c}$ crossed at a single 
point in the compression- $\tilde{\kappa}_{\text {eff }}$ parameter space. We identified the parameters of the simulation closest to this crossing point and considered these to be the simulation parameters on which the experimental values map. We considered only the linear modulus and the critical strain in the mapping and not the onset strain, because the error in obtaining this value is big compared to the relevant range for mapping. This procedure was performed for composite networks of hyaluronan with telocollagen and with atelocollagen (Figure 4.A.11). We observed significantly higher compression values for atelocollagen compared to telocollagen, consistent with the predictions from the double network model (Figure 4.3(d)). In contrast, the values for $\tilde{\kappa}_{\text {eff }}$ are comparable above a hyaluronan concentration of $3 \mathrm{mg} \mathrm{ml}^{-1}$, indicating that for both types of collagen the linear modulus is dominated by the hyaluronan matrix above this concentration. Below $3 \mathrm{mg} \mathrm{ml}^{-1}$, the scaling of the linear modulus with the hyaluronan concentration is absent or very weak, indicating that here the linear modulus is dominated by the collagen fibre network.

\subsection{Results and discussion}

To understand the interplay between elastic effects and internal stress in the mechanical response of tissues, we reconstitute biomimetic composites from two paradigmatic biopolymers: collagen and hyaluronan (Figure 4.1(a)). Collagen type I is the most ubiquitous member of the collagen family ${ }^{9}$, and hyaluronan is abundant in healthy tissues and upregulated in solid tumours ${ }^{32}$. Rheology measurements show that collagen and hyaluronan individually respond rather differently to an applied shear strain (Figure 4.1(b)). Collagen networks stiffen with strain above a threshold of about $5-10 \%$, as expected from their subisostatic fibrous architecture. By contrast, crosslinked hyaluronan networks exhibit a mechanical response that to a good approximation is linear in the range of strains that we consider (Figure 4.1(b)). Unlike collagen, hyaluronan polymers are flexible, with a persistence length of only $5-10 \mathrm{~nm}^{33}$. Their elastic properties are, therefore, determined by the chain entropy and the network response is linear until the long chains are pulled taut.

Having established the individual network response, we generate composites by polymerizing collagen monomers in the presence of a mixture of hyaluronan chains and PEGDA crosslinkers. Collagen polymerization and hyaluronan crosslinking occur concurrently, resulting in an interpenetrating double network. The final collagen network looks almost identical to its pure collagen counterpart and hyaluronan forms a background which is uniform over length scales much smaller than the collagen network mesh size, suggesting that the two networks do not interact, as confirmed by biochemical assays. ${ }^{20}$ 
To test the impact of hyaluronan on the strain-stiffening response of collagen, we perform rheology measurements on composites with a fixed $1 \mathrm{mg} \mathrm{ml}^{-1}$ collagen concentration and hyaluronan concentrations $c_{\mathrm{HA}}$ ranging from 0 to $7 \mathrm{mg} / \mathrm{mL}$. The composites strain-stiffen, but with a strong dependence on the hyaluronan content (Figure 4.1(b)). The addition of hyaluronan results in a striking enhancement of the linear elastic modulus $G_{0}$ beyond the sum of the moduli of the individual components (Figure 4.1(c)). This synergistic enhancement appears to be at a maximum around $4 \mathrm{mg} / \mathrm{mL}$, beyond the concentration at which hyaluronan first forms a percolating network. ${ }^{20}$ In the non-linear regime, the composite

(a)

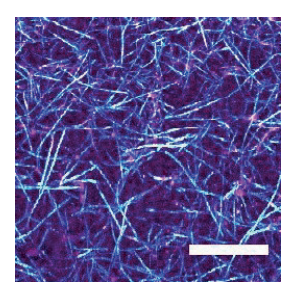

(c)
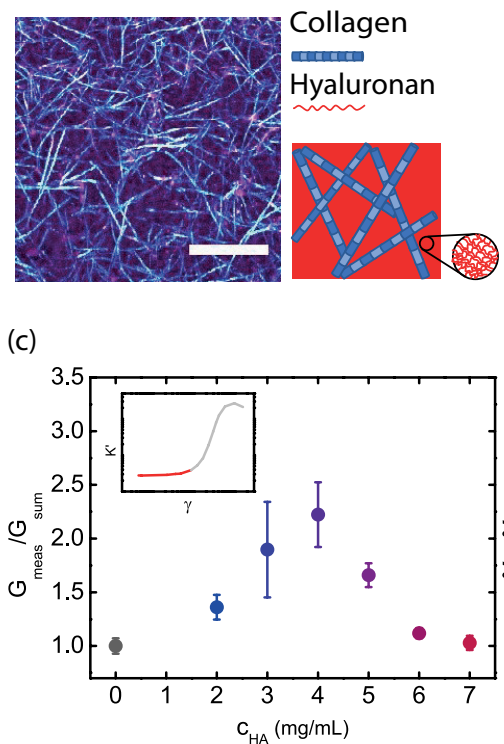

(b)

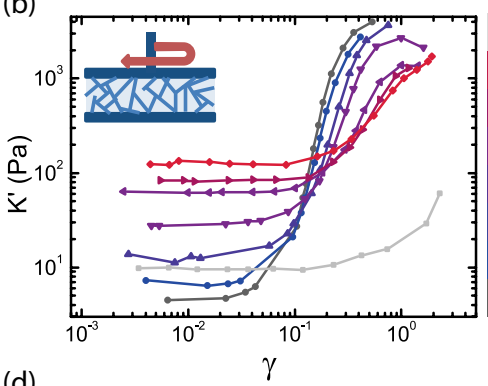

(d)

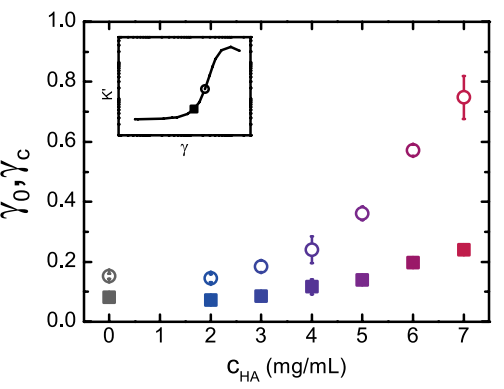

Figure 4.1. Composite collagen-hyaluronan networks as a minimal tissue-mimetic model system. (a) Confocal image together with a schematic of a composite network composed of a fibrous scaffold of collagen mixed with a soft hyaluronan gel that fills the interstices $\left(c_{\mathrm{col}}=1 \mathrm{mg} \mathrm{ml}^{-1}, c_{\mathrm{HA}}=\right.$ $4 \mathrm{mg} \mathrm{ml}^{-1}$ ). (b) Differential elastic modulus, $K^{\prime}$, as a function of applied strain for composites with a fixed concentration of collagen $\left(1 \mathrm{mg} \mathrm{ml}^{-1}\right)$ and varying hyaluronan concentration (coloured curves). For comparison, pure collagen is shown in dark grey and hyaluronan $\left(4 \mathrm{mg} \mathrm{ml}^{-1}\right)$ in light grey. Inset: the network is probed by shearing between two parallel plates. (c) Linear mechanical enhancement and (d) onset strain ( $\gamma_{o}$, solid symbols) and critical strain ( $\gamma_{c}$, open symbols) as a function of hyaluronan concentration, plotted with the same colour code as in (b). Data represent averages with standard error of the mean for at least three independent measurements. 
networks have a strongly delayed stiffening response compared to pure collagen (Figure 4.1(d)). Both the onset strain $\gamma_{0}$, where non-linearity first sets in, and the critical strain $\gamma_{c}$, where collagen fibres complete the transition from a bendingto stretching-dominated mode of deformation, shift upwards with increasing hyaluronan concentration (Figure 4.1(d)). In pure collagen networks, one important factor influencing the onset of strain-stiffening is the network connectivity ${ }^{8,22}$. To test whether hyaluronan impacts the connectivity of collagen networks, we perform delayed crosslinking experiments, adding PEGDA crosslinks only after collagen polymerization is complete. As the mechanics of the composite networks is very similar irrespective whether hyaluronan is crosslinked during or after collagen polymerization ${ }^{20}$, we conclude that the delayed strain-stiffening of composites is not caused by changes in collagen network connectivity.

Another important determinant of the onset of strain-stiffening in collagen networks is mechanical stress $7,8,30,34$. To test whether hyaluronan causes any build-up of stresses during network formation, we measure the time-dependence of the normal stress $\sigma_{N}$ that the composite system exerts on the top rheometer plate as it assembles (Figure 4.2(a) and (b)). Initially ( $t=0 \mathrm{~min}$ ), collagen monomers and hyaluronan chains form a viscous solution. As collagen starts to assemble in fibres (Figure 4.2(a)) and hyaluronan starts to crosslink ( $t=20 \mathrm{~min}$ ), $G_{0}$ and $\sigma_{N}$ increase simultaneously (Figure 4.2(b)). The positive sign of the normal stress indicates swelling of the polymerizing gel. As hyaluronan continues crosslinking and collagen continues polymerizing $(t=60 \mathrm{~min})$, the normal force reverses and decreases, eventually reaching negative values, indicating gel contraction. A similar normal stress evolution is observed for hyaluronan alone, whereas collagen develops no observable normal stress (Figure 4.A.1). We interpret the normal force build-up as a competition between the electrostatic repulsion of the charged hyaluronan polymers and the intrinsic tendency of polymer chains to contract to maximize their configurational entropy. At the beginning of the crosslinking process, the electrostatic interactions dominate, causing the system to swell. As crosslinking progresses, the fraction of crosslinked hyaluronan chains increases and the associated contractile forces eventually take over. To investigate whether the contractile stresses that develop in the hyaluronan gel are transmitted to the collagen network, we performed localized laser ablation experiments, using a high intensity laser to disrupt the hyaluronan meshwork in the interstices of the collagen network. Localized removal of hyaluronan caused an immediate recoil of neighbouring collagen fibres, indicating the release of mechanical stress (Figure 4.2(d)-(e)). Altogether, our observations seem to suggest that the hyaluronan matrix indeed exerts compressive stresses on the collagen fibres, as sketched in Figure 4.2(f). 
To test whether the internal stress generated by hyaluronan can explain the altered strain-stiffening response of composite networks, we use two-component network simulations. We model collagen as a sub-isostatic network of fibre segments of length $\ell_{0,1}$ with bending and stretching energy proportional to the constants $\kappa$ and $\mu_{1}$, respectively, while hyaluronan is modelled as a homogeneous mesh of springs of length $\ell_{0,2}$ and stretching energy proportional to $\mu_{2}$ (Figure 4.3(a)). To assess the effect of increasing hyaluronan concentration, we first vary the matrix stiffness $\mu_{2}$. We observe an increase of the linear modulus of the composite networks

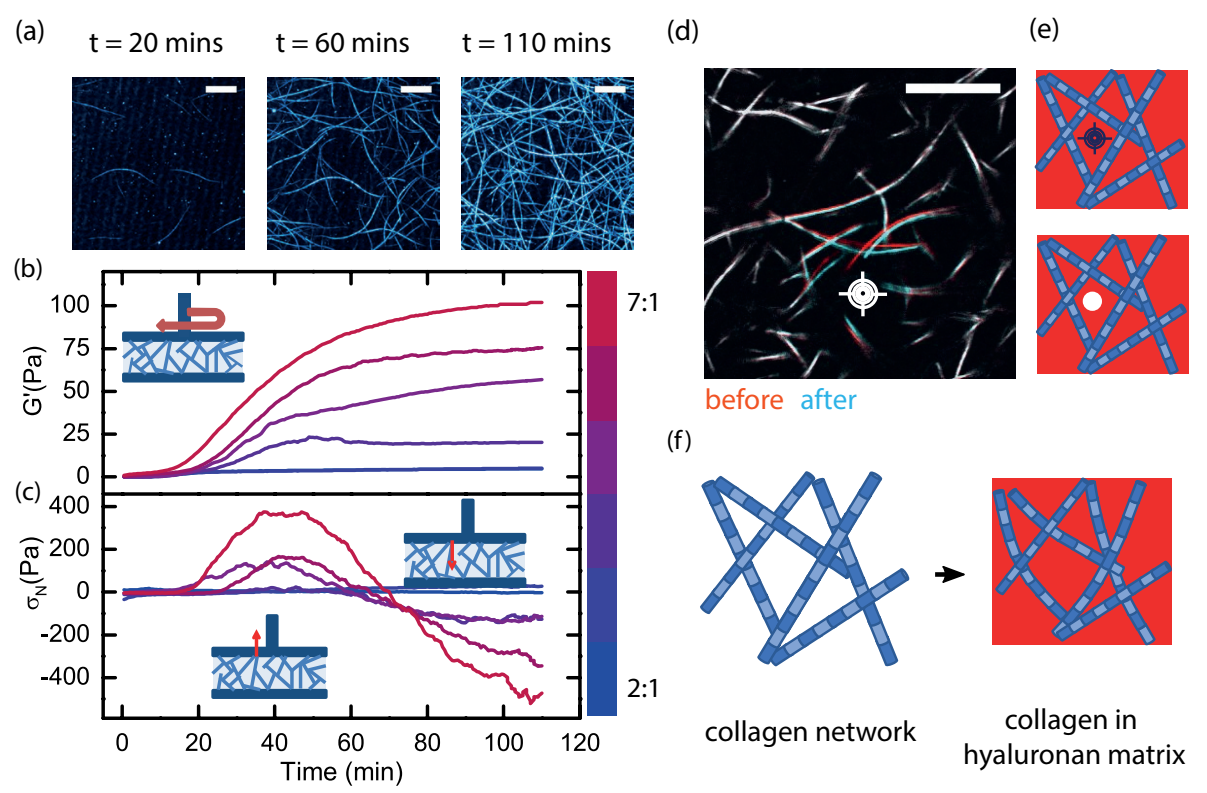

Figure 4.2. The hyaluronan gel prestresses the collagen network during gelation. (a) Z-projected confocal reflectance images of a composite network during the gelation process at three different time points, showing the formation of collagen fibres. Scale bars indicate $10 \mu \mathrm{m}$. (b) Evolution of the linear elastic modulus and (c) the corresponding normal force during network gelation for samples with a fixed collagen concentration $\left(1 \mathrm{mg} \mathrm{ml}^{-1}\right)$ and different hyaluronan concentrations (from $2 \mathrm{mg} \mathrm{ml}^{-1}$ to $7 \mathrm{mg} \mathrm{ml}^{-1}$ ). Note that the normal stress first increases, indicative of a swelling pressure, but afterwards decreases and reaches negative values, indicative of contractile stress. (d) Superposed confocal images taken before (red) and after (blue) localized ablation of the hyaluronan matrix (target) with a strong laser, schematically shown in (e). The collagen fibres are everywhere immobile (visible as white) except in the vicinity of the laser spot, where they recoil. Scale bar is $5 \mu \mathrm{m}$. (f) Our data suggest that hyaluronan forms a contractile matrix that causes the collagen fibres to bend. 
with increasing $\mu_{2}$, but no changes in the non-linear behaviour (Figure 4.A.4). Therefore, we include the contractile tendency of hyaluronan by reducing the

(a)

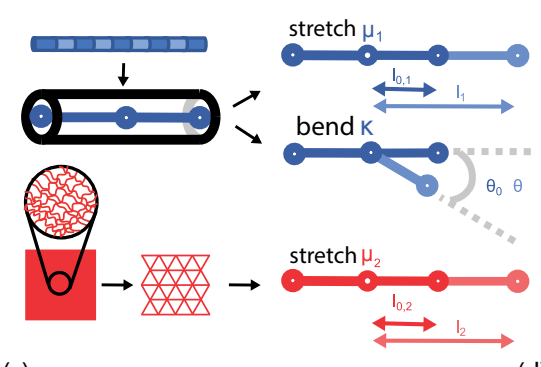

(c)

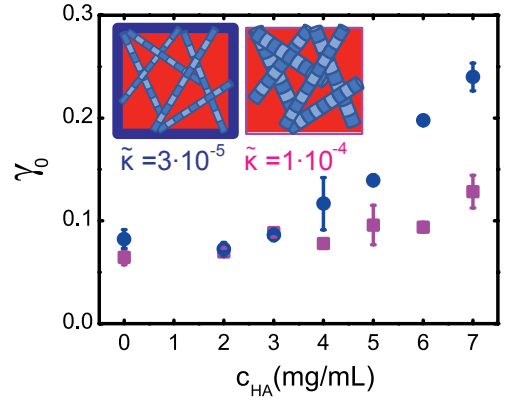

(b)

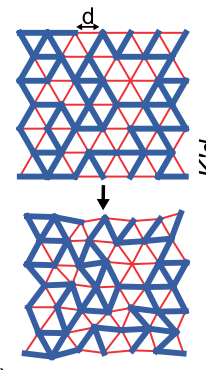

(d)
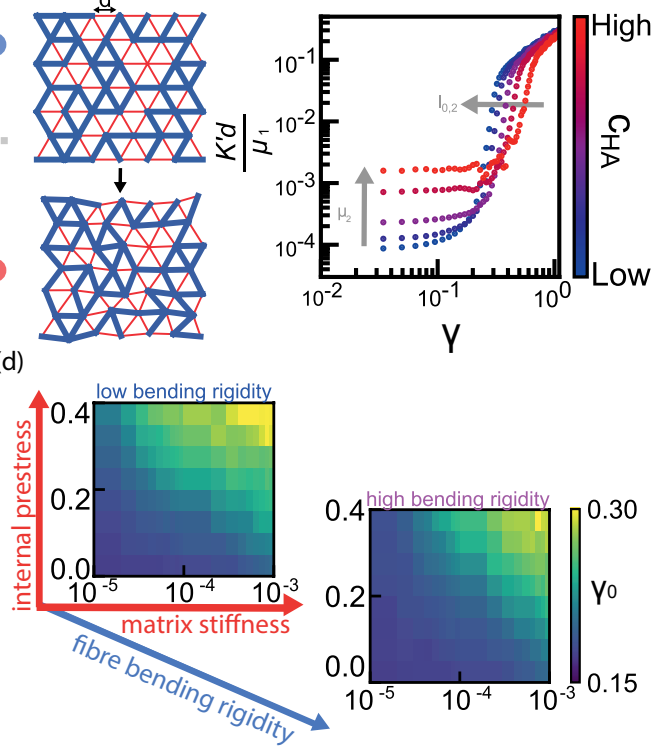

Figure 4.3. Computational modelling of two-component networks reveals that the composite mechanics depends on a balance between collagen bending and hyaluronan contraction. (a) The initial configuration is a sub-marginal fibre network (blue segments with stretching and bending resistance) embedded in a homogeneous flexible matrix (red segments, resistance to stretching only). To mimic the effect of hyaluronan contraction, we decrease the rest length $\ell_{0,2}$ of the matrix springs and reach mechanical equilibrium by allowing the network to compress.

(b) Shear modulus as a function of strain for composite networks with varying matrix stiffness $\mu_{2}$ (from $1 \times 10^{-5}$ to $5 \times 10^{-4}$ ) and varying internal pre-stress $\ell_{0,2}$ (from 1.00 to 0.60 ). We qualitatively recover the experimentally observed dependence on hyaluronan concentration when we allow for a simultaneous increase in matrix stiffness and in compressive force. In this set of simulations, the average connectivity $\langle z\rangle=3.4$ and the (rescaled) fibre bending stiffness $\tilde{\kappa}=3 \times 10^{-5}$ are kept fixed to the corresponding experimental values of the collagen network. (c) Experimentally measured onset strain for telocollagen (magenta squares) and atelocollagen (blue circles) networks as a function of hyaluronan concentration. (d) In simulations, $\gamma_{o}$ can be tuned by varying the collagen fibre rigidity and by independently varying the stiffness and internal stress of the hyaluronan gel, whereas experimentally the two matrix-related effects are inherently coupled when we vary hyaluronan concentration. 
rest length of the matrix springs $\ell_{0,2}$. Subsequently, we allow the entire system to establish a mechanical equilibrium between the contracting stresses in the hyaluronan matrix and the resisting bending forces of the collagen network by allowing the system to compress (Figure 4.3(a) and Ref. [20]). Inducing compression by lowering $\ell_{0,2}$ while keeping the matrix stiffness $\mu_{2}$ fixed has little effect on the linear regime, but delays the onset of strain stiffening (Figure 4.A.4). When we simultaneously increase $\mu_{2}$ to account for elastic reinforcement and lower $\ell_{0,2}$ to account for internal stress build-up, we recover both the linear mechanical enhancement and the delay in strain-stiffening (Figures 4.3(b), 4.A.4, and 4.A.5).

Our model suggests that hyaluronan tunes the mechanics of collagen networks through a combination of elastic reinforcement and internal prestress. This implies that the synergistic mechanics of the composites should be highly dependent on the fibre rigidity $\tilde{\kappa}$ (the ratio between $\kappa$ and $\mu_{1}$ ). Specifically, fibres more resistant to bending (larger $\tilde{\kappa}$ ) should be less sensitive to stresses arising from the background matrix. Furthermore, they should be less sensitive to the elastic reinforcement, given their already large bending rigidity. To test this prediction, we use a different type of collagen that forms stiffer fibres thanks to molecular crosslinking mediated by non-helical peptide extensions of the triple helix known as telopeptides ${ }^{35}$. By fitting the mechanical response curves to an analytical expression for fibrous networks ${ }^{22}$, we indeed find the fibre rigidity for the telocollagen to be $\tilde{\kappa}=1 \times 10^{-4}$, larger than for the atelocollagen considered so far, where $\tilde{\kappa}=3 \times 10^{-5}$ (Figure 4.A.7). The strain-stiffening behaviour of networks formed by telocollagen is indeed less affected by hyaluronan, as compared to the atelocollagen, with smaller changes in the onset and critical strain, as well as smaller changes in the linear mechanical enhancement in the linear regime (Figure 4.3(c) and Ref. [20]). Therefore, the mechanical properties of the composite system exhibit a phase space governed by a balance between the bending rigidity of collagen and the dual effect of the hyaluronan matrix on linear elasticity and internal stress (Figures 4.3(d) and 4.A.8).

Finally, we test whether the mechanical response of the double networks can be mapped onto an effective single-component model, as recently proposed for the linear elastic regime ${ }^{14}$. Our experiments suggest that this mapping should extend to the non-linear elastic regime, as we can rescale the strain-stiffening curves of composites onto the curve for pure collagen by normalizing the non-linear modulus with $G_{0}$ and the shear stress with an effective stress $\sigma_{\text {eff }}$ (Figure 4.4(a) Ref. [20]), which is manually computed and allows to overlap the curves. To capture the elastic reinforcement of collagen by the surrounding hyaluronan matrix in a single-component model, we assign an enhanced bending rigidity $\tilde{\kappa}_{\text {eff }}$ to the fibres, which accounts for the hindrance of fibre bending by the matrix ${ }^{14}$. Internal stresses are implemented by compressing the network and resetting the bending 
rest angles (Figures 4.4(b), 4.A.9, and 4.A.10). This simple model allows us to quantitatively map all experimental data for both atelocollagen and telocollagen networks onto a mechanical phase space controlled by $\tilde{\kappa}_{\text {eff }}$ and the level of collagen compression (Figures 4.4(c), 4.A.10, and 4.A.11). The mapping reveals that the compression is significantly higher for atelocollagen compared to telocollagen, consistent with the more elaborate double network model (Figure 4.3(d)). We furthermore find that the parameters controlling the linear and the non-linear regime are decoupled, with the linear modulus $G_{0}$ depending on $\tilde{\kappa}_{\text {eff }}$ and the critical strain $\gamma_{c}$ on the extent of compression.

\subsection{Concluding remarks}

We showed by experiments on tissue-mimetic composites that the combination of fibrous collagen networks with a soft polysaccharide hydrogel results in highly tuneable non-linear mechanics. The soft gel tunes the mechanics by introducing both elastic reinforcement and internal stresses. The stress-induced stiffening we

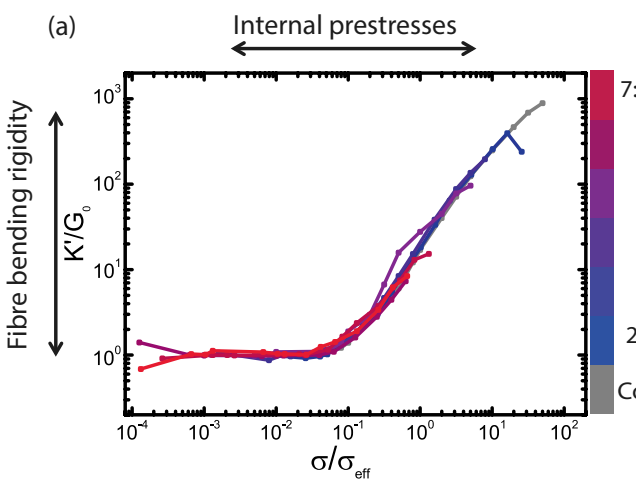

(b)

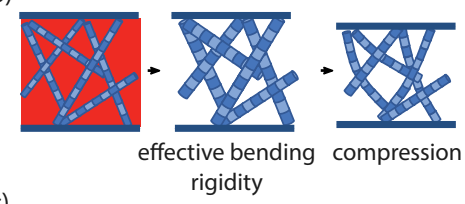

(c)

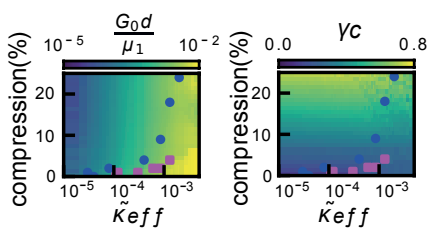

Figure 4.4. The mechanical response of composite networks can be mapped onto an effective single-component model. (a) The strain-stiffening curves of composite networks with varying hyaluronan concentration can be rescaled on top of the stiffening curve of a pure collagen network (grey) by normalizing the shear stress by an effective stress $\sigma_{\text {eff }}$ and the non-linear modulus by $G_{0} . \sigma_{\text {eff }}$ is manually determined in order to be able to overlap the curves of the composites with the ones for collagen. ${ }^{20}$ The collagen concentration is fixed at $1 \mathrm{mg} \mathrm{ml}^{-1}$. (b) The matrix effects can be incorporated in a single-component system by changing two properties of the collagen network: replacing the bending rigidity with an effective bending rigidity and compressing the network. (c) Map of $G_{0}$ and $\gamma_{c}$ as a function of $\tilde{\kappa}_{\text {eff }}$ and compression. The symbols (magenta squares for telocollagen and blue circles for atelocollagen) indicate the quantitative mapping of the experimental results on the single-component model. 
observe is reminiscent of the active stiffening of cytoskeletal and extracellular matrix networks by contractile molecular motors or cells ${ }^{36-38}$, but with the distinction that here energy consumption is not required. The minimal computational model we developed can be applied also to other soft tissues such as the plant cell wall, where a rigid cellulose network is combined with a soft pectin matrix ${ }^{39}$. Our findings not only elucidate how biology combines biopolymers with complementary properties to finely-tune the mechanics of living tissues, but also provide a new avenue for the design of synthetic elastic materials. The bio-inspired concept of combining semiflexible and flexible polymer networks to improve mechanical properties has been recently translated into material science ${ }^{40}$. Here, we showed that internal stress generation introduces a powerful control knob to tune the mechanical response of a material. This principle is irrespective of the nature of the stress source, and can, therefore, also be implemented in synthetic materials by using $\mathrm{pH}$ - or temperature-responsive components.

\section{Acknowledgements}

This chapter is part of a collaboration with Federica Burla and Gijsje Koenderink (AMOLF, Amsterdam) who led the experimental part of this project. We thank Fred MacKintosh (Rice University, Texas) for useful discussions. 


\section{A Appendix}

\section{A.1 Double network initialization}

Initialization of double networks takes place in three sequential steps:

1. Generation of the network geometry: The collagen network is represented by a fibre network modelled on a triangular lattice composed of $L$ by $L$ nodes with lattice spacing $d$. We simulate on lattices of $L=50$. A bond dilution procedure is designed to mimic the connectivity of collagen networks, where either two fibres meet at a node, resulting in a 3-fold branching point, or two fibres cross, resulting in a 4 -fold crosslink. ${ }^{22,24}$ Thus, the maximum connectivity at every node should be 4 as opposed to 6 for a regular undiluted triangular lattice. To fulfil this requirement, we use the following procedure (an adaptation of other recent simulations of collagen networks ${ }^{23}$ ): 1) we make a list of all the nodes with more than 4 connecting segments, 2) randomly choose a node in this list, 3) randomly remove one segment connecting to this node. We repeat this procedure until all nodes have a maximum of 4 connecting segments. Successively, we further dilute the network to reach the desired average connectivity $\langle z\rangle$. Here we perform simulations on networks with $\langle z\rangle$ $=3.4$. All segments of the fibre network have a rest length equal to the lattice spacing $d$, and stretching or compression of these segments costs energy. In addition, all segments that are connected to each other in a straight line have a bending contribution. The hyaluronan matrix is instead represented by a homogeneous network of springs superposed on the same triangular lattice used to generate the submarginal fibre network. The fibre and matrix segments share the same nodes. Since the matrix segments are never diluted, every node has at least 6 matrix bonds connected to it and up to 4 fibre bonds. An example of a typical two-dimensional composite network obtained with this procedure is shown in Figure 4.A.3.

2. Implementation of internal stresses before shear simulations: To model the contractile stresses introduced by hyaluronan during the crosslinking process, we reduce the rest length $\ell_{0,2}$ of the segments of the homogeneous spring network, which initially equals $d$, in steps of $0.1 d$. After every step, the energy of the complete system is minimized including both the fibre and the matrix segments.

3. Mechanical equilibration before shear simulations: Step 2 brings the system out of mechanical equilibrium. We define mechanical equilibrium as a situation in which the system would retain its shape if the boundaries were removed, 
or equivalently any small deformation would increase the total energy of the system. To re-establish mechanical equilibrium, we change the size of the simulation box. Specifically, we need to make the box smaller, to accommodate for the contractile tendency of the matrix. We, therefore, isotropically shrink the box in steps of $0.01 \%$ bulk strain. After every step the energy is minimized. As a result, the matrix relaxes, but the fibres bend in order to still fit in the reduced volume, as shown in Figure 4.A.3. At small bulk strains, the increase in bending energy is outweighed by the decrease in matrix stretching energy. However, once the bulk strain reaches high enough values, the increase in bending energy dominates. These two opposing tendencies lead to a minimum in the total energy, as shown in Figure 4.A.4. The corresponding compression is expressed as $\left(L_{\text {init }}-L_{\text {min }}\right) / L_{\text {init }}$, where $L_{\text {init }}$ is the initial length of one of the axes of the simulation box and $L_{\min }$ is the length once the energy minimum is reached. The amount of compression needed to achieve mechanical equilibrium depends on the stiffness and the reduction of the segment length in the matrix (Figure 4.A.3) as well as on the fibre bending rigidity.

To obtain the mechanical response of the composite networks, we apply a shear strain up to $150 \%$ in steps of $0.1 \%$. We use experimentally relevant values for the bending rigidity based on fits of a fibrous network model ${ }^{22}$ to the strain-stiffening curves measured for atelocollagen $\left(\tilde{\kappa}=3 \times 10^{-5}\right)$ and telocollagen $\left(\tilde{\kappa}=1 \times 10^{-4}\right)$ networks. Since the experimental results show that the linear moduli of pure hyaluronan and collagen are of the same order of magnitude, we choose parameters in the simulations such that also here the linear moduli of the individual components are comparable (Figure 4.A.4).

To calculate the modulus versus strain curves, the energy density versus strain curves are smoothened (running average with a range of $1 \%$ strain) and 100 equally spaced points are sampled via linear interpolation. The first derivative of this curve gives the stress, while the second derivative provides the modulus as a function of strain. From these data, the linear modulus $G_{0}$, onset strain for stiffening $\gamma_{0}$, and critical strain $\gamma_{c}$ are obtained for every individual curve. Data shown are averages over $N_{\text {config }} \sim 10$ samples.

\section{A.2 Force equations used in the energy minimization algorithm}

Energy minimization for our model is performed using the recently introduced FIRE algorithm that uses expressions for the force to find the minimum in the energy ${ }^{25}$. Here we present the equation for the stretching force and derive the equations for the different force components caused by bending the fibres. Nodes 
$i$ of the network have coordinates $\boldsymbol{x}_{i}=\left[x_{i, 1}, x_{i, 2}\right]$ and are connected by segments $\boldsymbol{r}_{i j}=\boldsymbol{x}_{j}-\boldsymbol{x}_{i}$. The force due to the stretching contribution acting upon node $\boldsymbol{x}_{i}$ along $\boldsymbol{r}_{i j}$ is defined as

$$
f_{\text {stretch, } \boldsymbol{x}_{i}}=-\frac{\mu_{1}}{\ell_{0,1}}\left(\left|\boldsymbol{r}_{i j}\right|-\ell_{0,1}\right) .
$$

The expression for the bending force depends on a set of three nodes $\boldsymbol{x}_{i}, \boldsymbol{x}_{j}, \boldsymbol{x}_{k}$. For each node, the force equation is found via the gradient of the bending energy $-\nabla_{\text {node }} E_{\text {bend }}$, where

$$
E_{\text {bend }}=\frac{\kappa}{2 \ell_{0,1}}(\Delta \theta)^{2}=\frac{\kappa}{2 \ell_{0,1}}\left(\theta-\theta_{0}\right)^{2} .
$$

To derive the force, we need an expression for $\theta$ as a function of the node coordinates $\boldsymbol{x}_{i}, \boldsymbol{x}_{j}, \boldsymbol{x}_{k}$. Here we use $\cos \theta=\frac{\boldsymbol{r}_{i j} \cdot \boldsymbol{r}_{j k}}{\left|\boldsymbol{r}_{i j}\right| \boldsymbol{r}_{j k} \mid}$. If $\theta=-\pi \ldots 0$,

$$
\theta_{-}\left(\boldsymbol{x}_{i}, \boldsymbol{x}_{j}, \boldsymbol{x}_{k}\right)=-\arccos \frac{\boldsymbol{r}_{i j} \cdot \boldsymbol{r}_{j k}}{\left|\boldsymbol{r}_{i j}\right|\left|\boldsymbol{r}_{j k}\right|}+\theta_{\text {cor }} .
$$

and the force reads

$$
f_{\text {bend }, x_{m, n}}=\frac{\frac{\kappa\left(\theta_{-}\left(\boldsymbol{x}_{i}, \boldsymbol{x}_{j}, \boldsymbol{x}_{k}\right)-\theta_{0}\right)}{\ell_{0, i j}+\ell_{0, j k}} g_{x_{m, n}}\left(\boldsymbol{x}_{i}, \boldsymbol{x}_{j}, \boldsymbol{x}_{k}\right)}{\sqrt{1-\left(-\frac{\boldsymbol{r}_{i j} \cdot \boldsymbol{r}_{j k}}{\left|\boldsymbol{r}_{i j}\right|\left|\boldsymbol{r}_{j k}\right|}\right)^{2}}}
$$

or if $\theta=0 \ldots \pi$,

$$
\theta_{+}\left(\boldsymbol{x}_{i}, \boldsymbol{x}_{j}, \boldsymbol{x}_{k}\right)=\arccos \frac{\boldsymbol{r}_{i j} \cdot \boldsymbol{r}_{j k}}{\left|\boldsymbol{r}_{i j}\right|\left|\boldsymbol{r}_{j k}\right|}-\theta_{\mathrm{cor}} .
$$

and the force reads

$$
f_{\text {bend }, x_{m, n}}=\frac{\frac{\kappa\left(\theta_{+}\left(\boldsymbol{x}_{i}, \boldsymbol{x}_{j}, \boldsymbol{x}_{k}\right)-\theta_{0}\right)}{\ell_{0, i j}+\ell_{0, j k}} g_{x_{m, n}}\left(\boldsymbol{x}_{i}, \boldsymbol{x}_{j}, \boldsymbol{x}_{k}\right)}{\sqrt{1-\left(\frac{\boldsymbol{r}_{i j} \cdot \boldsymbol{r}_{j k}}{\left|\boldsymbol{r}_{i j}\right| \boldsymbol{r}_{j k} \mid}\right)^{2}}}
$$

Here, $\theta_{\text {cor }}$ is equal to $2 \pi$ if $\left|\theta-\theta_{0}\right|$ is larger than $\pi$ and is 0 otherwise. The functions $g_{x_{m, n}}$ are specific for the node and the component of the force that is considered:

$$
g_{x_{i, n}}=\frac{\left(x_{j, n}-x_{i, n}\right)\left(\boldsymbol{r}_{i j} \cdot \boldsymbol{r}_{j k}\right)}{\left|\boldsymbol{r}_{i j}\right|^{3}\left|\boldsymbol{r}_{j k}\right|}+\frac{x_{j, n}-x_{k, n}}{\left|\boldsymbol{r}_{i j}\right|\left|\boldsymbol{r}_{j k}\right|}
$$




$$
\begin{aligned}
& g_{x_{j, n}}=\frac{\left(x_{i, n}-x_{j, n}\right)\left(\boldsymbol{r}_{j i} \cdot \boldsymbol{r}_{j k}\right)}{\left|\boldsymbol{r}_{i j}\right|^{3}\left|\boldsymbol{r}_{j k}\right|}+\frac{x_{k, n}+x_{i, n}-2 x_{j, n}}{\left|\boldsymbol{r}_{i j}\right|\left|\boldsymbol{r}_{j k}\right|}+\frac{\left(x_{k, n}-x_{j, n}\right)\left(\boldsymbol{r}_{j i} \cdot \boldsymbol{r}_{j k}\right)}{\left|\boldsymbol{r}_{i j}\right|\left|\boldsymbol{r}_{j k}\right|^{3}} \\
& g_{x_{k, n}}=\frac{\left(x_{j, n}-x_{k, n}\right)\left(\boldsymbol{r}_{j i} \cdot \boldsymbol{r}_{j k}\right)}{\left|\boldsymbol{r}_{i j}\right|\left|\boldsymbol{r}_{j k}\right|^{3}}+\frac{x_{j, n}-x_{i, n}}{\left|\boldsymbol{r}_{i j}\right|\left|\boldsymbol{r}_{j k}\right|}
\end{aligned}
$$

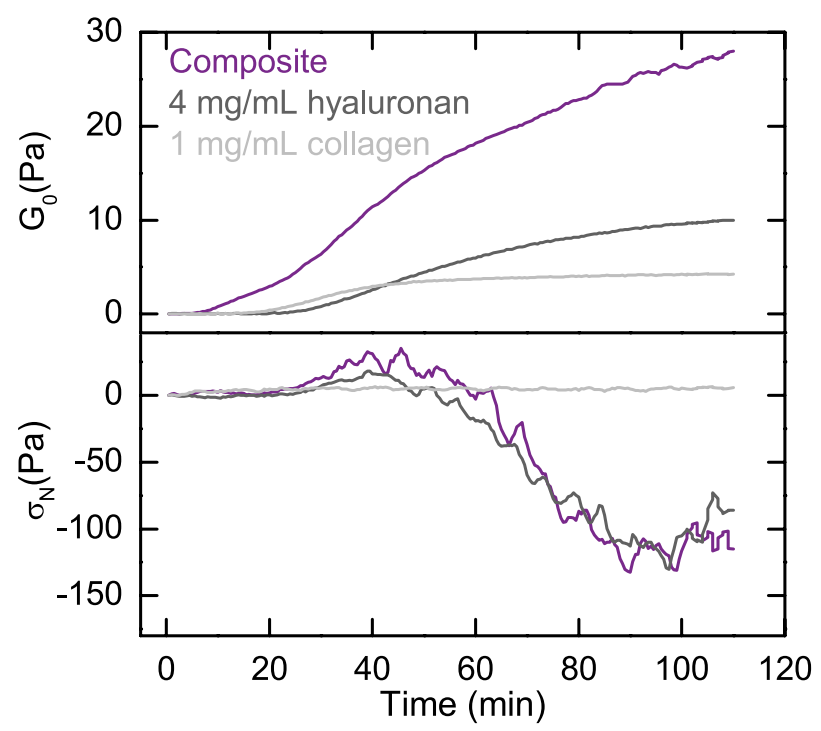

Figure 4.A.1. Polymerization curves and normal stress evolution for pure collagen, pure hyaluronan, and composite system. Top panel: Time evolution of the linear elastic shear modulus during polymerization for a composite system composed of $1 \mathrm{mg} \mathrm{ml}^{-1}$ collagen and $4 \mathrm{mg} \mathrm{ml}^{-1}$ hyaluronan (purple, top-most curve), pure collagen at $1 \mathrm{mg} \mathrm{ml}^{-1}$ (light grey), and pure hyaluronan $4 \mathrm{mg} \mathrm{ml}^{-1}$ (dark grey). Bottom panel: Corresponding time evolution of the normal stress as a function of polymerization time. Note that no measurable normal stress is observed in the pure collagen system. By contrast, the normal stress for the pure hyaluronan network and the collagen-hyaluronan composite first increases and then decreases, finally reaching a negative value indicative of contractile prestress. 
(a)

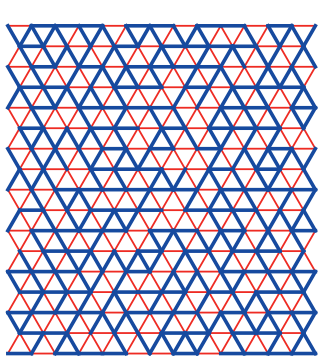

(b)

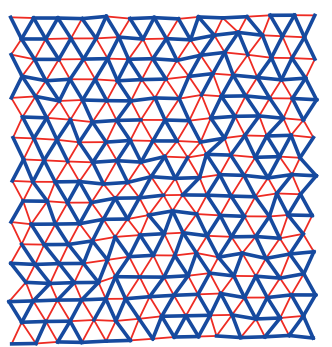

(c)

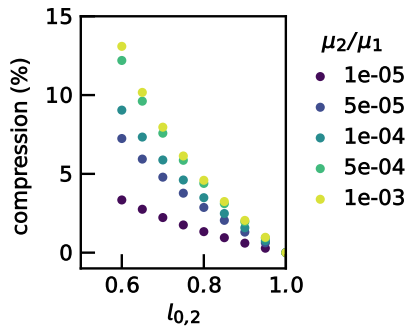

(d)

山

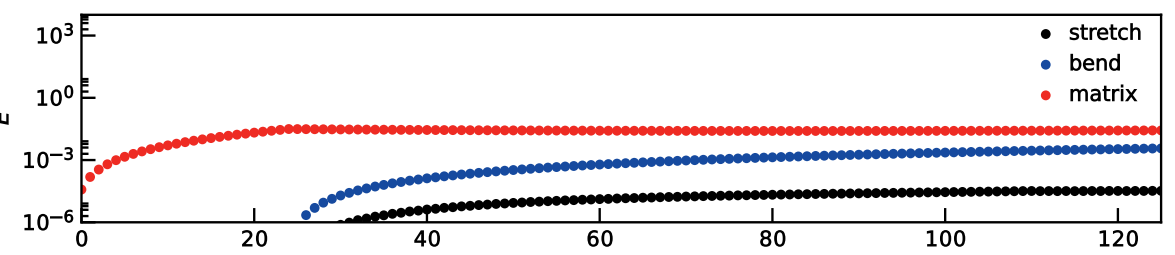

(e)

Simulation steps

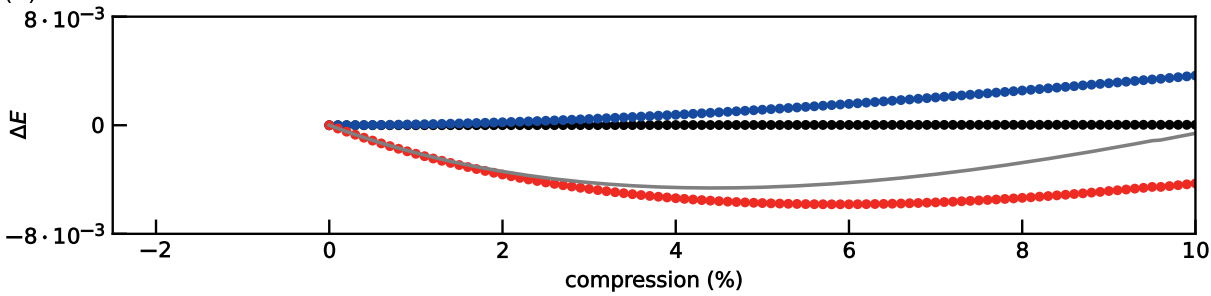

Figure 4.A.2. Two-component simulations: network initialization. Snapshots of a double network before (a) and after (b) bulk compression by $3.4 \%$ bulk strain. (c) Equilibrium values of bulk compression as a function of $\ell_{0,2}$ for $\tilde{\kappa}=3 \times 10^{-5}$, shown for different values of $\mu_{2} / \mu_{1}$. (d) Total energy of the composite system as a function of the number of simulation steps during compression, decomposed in the stretching and bending energy of the collagen network and the stretching energy associated with the hyaluronan matrix. (e) Energy change upon bulk compression with respect to the reference state $\left(0 \%\right.$ bulk compression, $\left.\ell_{0,2}=0.75\right)$. The total energy is indicated with a grey line. The equilibrium state corresponds to the state of minimum energy. The panel shows an example obtained for a diluted triangular network with $\langle z\rangle=3.4$, $L=50, \tilde{\kappa}=3 \times 10^{-5}, \tilde{\mu}_{2}=3 \times 10^{-5}$ and $\ell_{0,2}=0.75 \mathrm{~d}$, where mechanical equilibrium occurs at a compression of $4.4 \%$. 
(a)

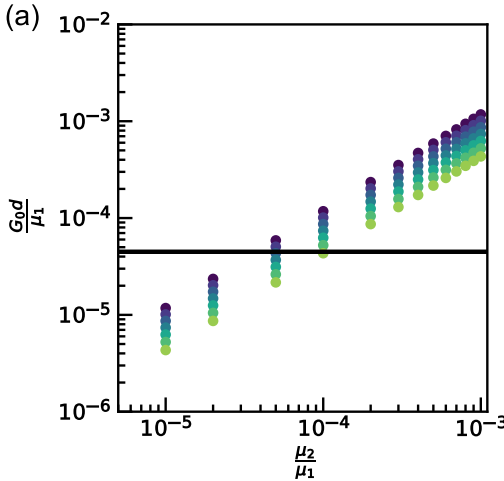

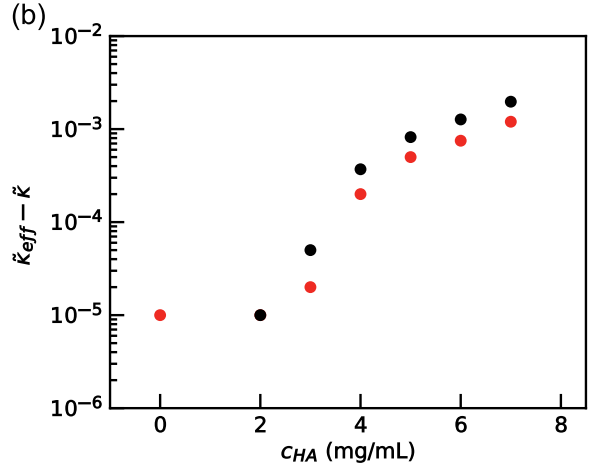

Figure 4.A.3. Choosing two-component simulation parameters to mimic experimental conditions. (a) Linear shear modulus for homogeneous triangular networks of springs as a function of $\mu_{2} / \mu_{1}$. The black line indicates the linear modulus of a diluted triangular lattice with average connectivity 3.4 and $\tilde{\kappa}=3 \times 10^{-5}$. (b) In the effective single component model, the effective bending rigidity ${ }^{14}$ follows the equation $\tilde{\kappa}_{\text {eff }}=\tilde{\kappa}+\tilde{\mu}_{2}$, hence $\tilde{\kappa}_{\text {eff }}-\tilde{\kappa}$ should be equal to $\tilde{\mu}_{2}$ when $d$ is equal to unity. In (b) the difference between the effective bending rigidity found in the mapping of the results for the composite networks and the bending constant for bare atelocollagen (red, $\tilde{\kappa}=3 \times 10^{-5}$ ) and telocollagen (black, $\tilde{\kappa}=1 \times 10^{-4}$ ) is shown. The matrix stiffness found in this way ranges from $10^{-5}$ to $10^{-3}$, which corresponds with the range of $\tilde{\mu}_{2}$ values explored in the double network simulations.

(a)

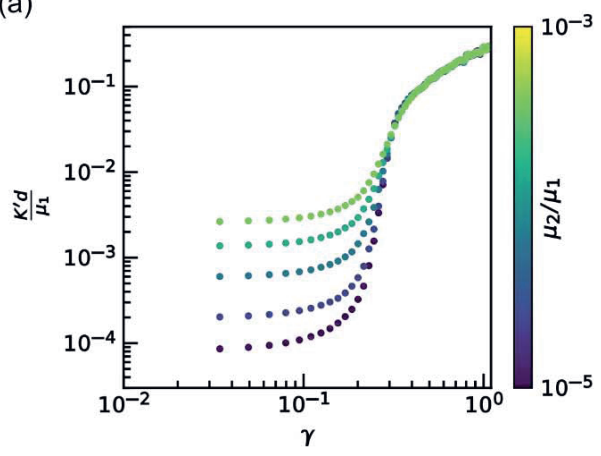

(b)

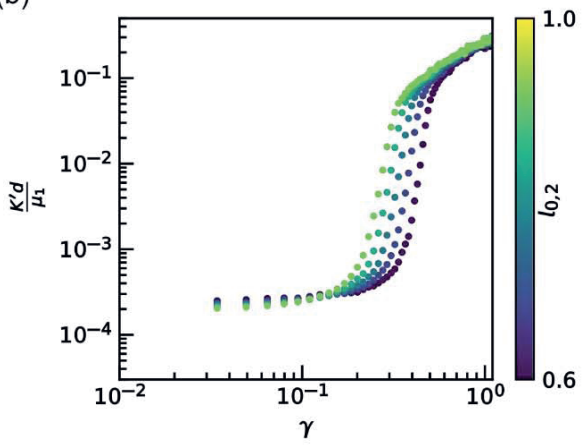

Figure 4.A.4. Simulated two-component stiffening response when varying independently matrix stiffness and matrix prestress. Modulus versus strain curves for (a) varying matrix stiffness at fixed $\ell_{0,2}=0.75 d$ and (b) varying matrix rest length at fixed $\mu_{2} / \mu_{1}=10^{-4}$. Results obtained using diluted triangular networks with $\langle z\rangle=3.4, L=50, N_{\text {config }}=20, \tilde{\kappa}=3 \times 10^{-5}$, and $\tilde{\mu}_{2}=$ $3 \times 10^{-5}$. 
(a)

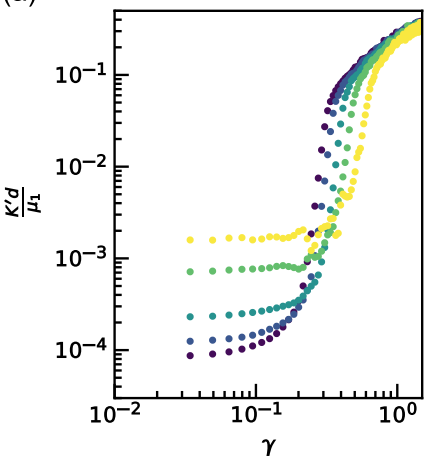

(c)

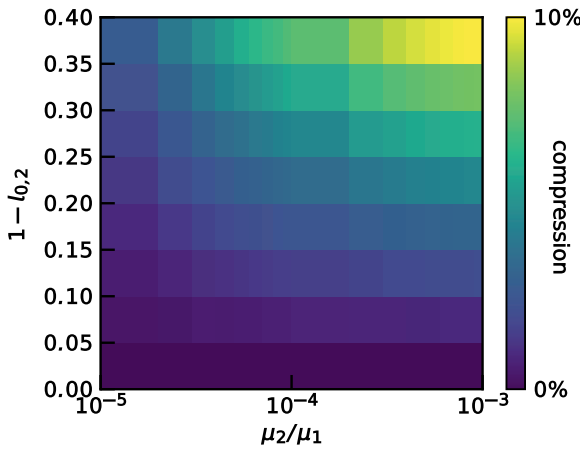

$\mu_{2} / \mu_{1} ; l_{0,2}$ (b)

- $1 \mathrm{e}-05 ; 1.00$

- $2 \mathrm{e}-05 ; 0.85$

- $5 \mathrm{e}-05 ; 0.75$

$2 \mathrm{e}-04 ; 0.70$

$5 e-04 ; 0.60$

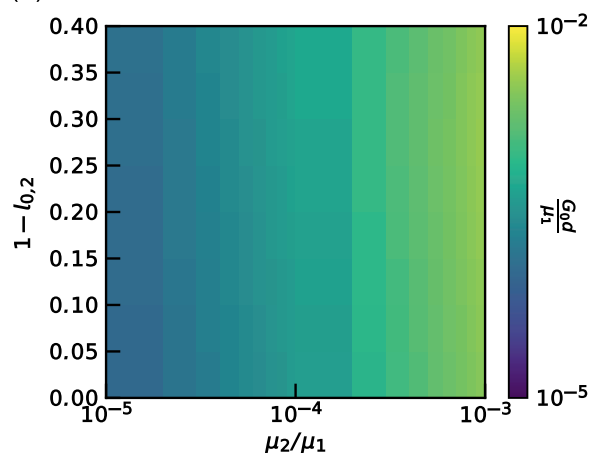

(d)

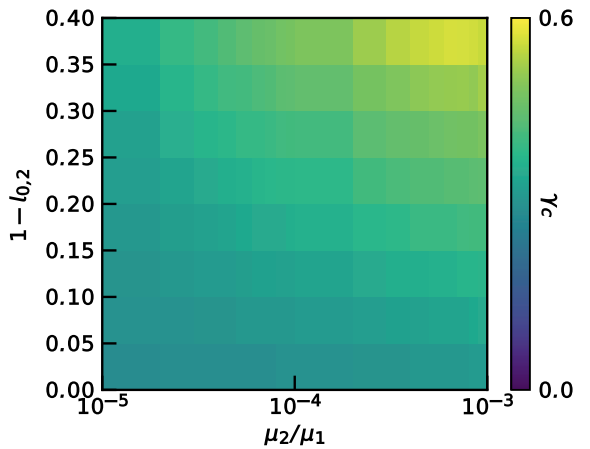

Figure 4.A.5. Mechanical properties of simulated two-component networks with $\tilde{\kappa}=3 \times 10^{-5}$, corresponding to atelocollagen. (a) Example of modulus versus strain curves obtained by changing simultaneously the stretching constant and the rest length of the hyaluronan matrix. (b-d) Phase space representations of the (b) linear elastic shear modulus, (c) bulk compressive strain at mechanical equilibrium, and (d) critical strain, shown as a function of matrix stiffness relative to fibre network stiffness and of the reduction of matrix segment rest length. 


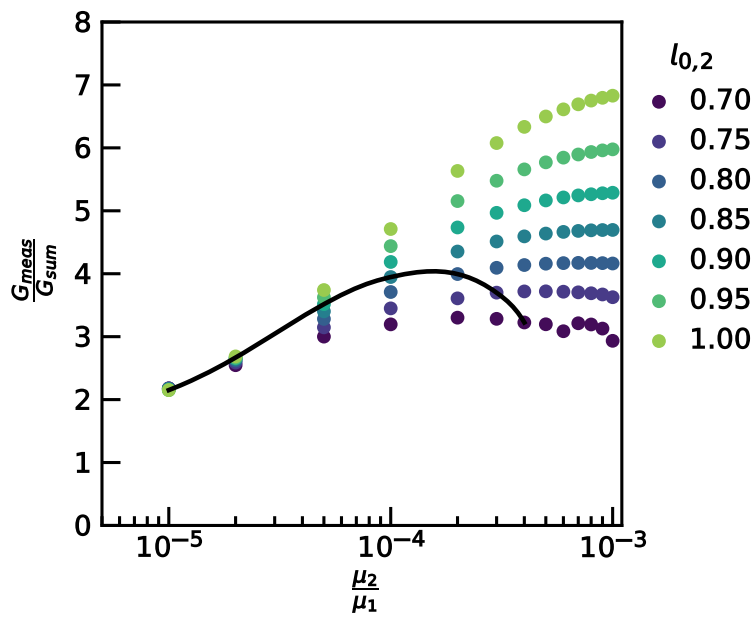

Figure 4.A.6. Linear mechanical enhancement in the double network simulations. The linear mechanical enhancement is calculated for the double network simulations as $G_{\text {meas }} / G_{\text {sum }}$, with $G_{\text {meas }}$ the modulus obtained in the double network simulations, and $G_{\text {sum }}$ obtained as the sum of the modulus of the fibre network $\left(\langle z\rangle=3.4, \tilde{\kappa}=3 \times 10^{-5}\right)$ and that of the matrix (homogeneous triangular lattice with stiffness $\left.\tilde{\mu}_{2}\right)$. Without internal stress $\left(\ell_{0,2}=1.00\right)$, the linear mechanical enhancement is proportional to the matrix stiffness. For small $\tilde{\mu}_{2}$, the linear mechanical enhancement depends weakly on the matrix rest length $\ell_{0,2}$. However, for large $\tilde{\mu}_{2}$ there is a strong dependence on $\ell_{0,2}$. Experimentally, a peak is observed in the linear mechanical enhancement as a function of $c_{\mathrm{HA}}$, as shown in Figure 4.1(c). Qualitatively, we observe a similar trend in the simulation data. We start with the notion that the mechanical response as a function of hyaluronan concentration matches qualitatively with a simultaneous increase of matrix stiffness and decrease of rest length in the experiment (Figure 4.3(b). From Figure 4.A.3(b) we can estimate that the stiffness of the matrix ranges from $10^{-5}$ to $10^{-3}$. In addition, the quantitative mapping predicts a compression of $10 \%$ at a hyaluronan concentration of $5 \mathrm{mg} \mathrm{ml}^{-1}$. In the double network simulations, this corresponds to $\ell_{0}$ values between 1.00 and 0.70 , as can be concluded from Figure 4.A.2(c). The black line shows that, if the rest length decreases with increasing stiffness roughly following these ranges, a peak in the mechanical enhancement is present. 
(a)

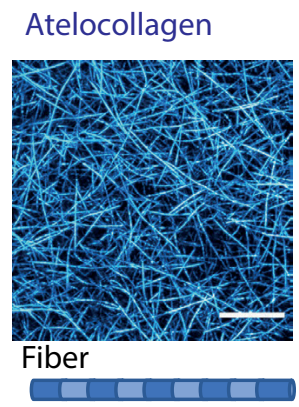

Monomer

500000000000000000000000
Telocollagen
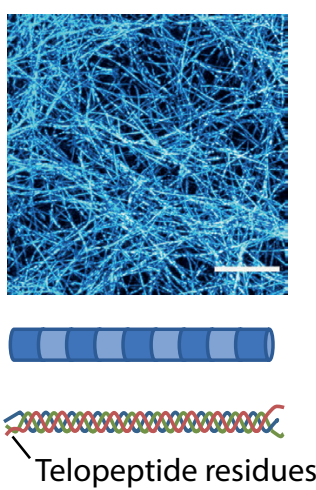

(b)

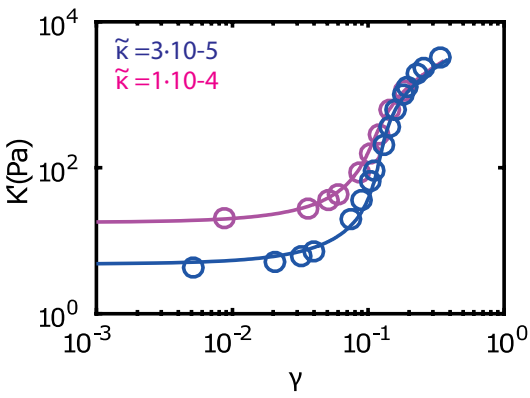

Figure 4.A.7. Changing the fibre rigidity by including or removing the telopeptide regions of collagen. (a) Z-stack projections of atelo and telocollagen networks $\left(1 \mathrm{mg} \mathrm{ml}^{-1}\right)$ imaged with confocal reflectance microscopy, together with schematics showing the difference between the two at the fibre level (different fibre diameter) and molecular level (absence or presence of telopeptides that mediate crosslinking between adjacent collagen monomers). (b) Fits (lines) of the athermal fibrous network model ${ }^{22}$ to the measured strain-stiffening curves of the two types of networks (circles) reveal a different dimensionless fibre rigidity for collagen and atelocollagen (inset). 
(a)

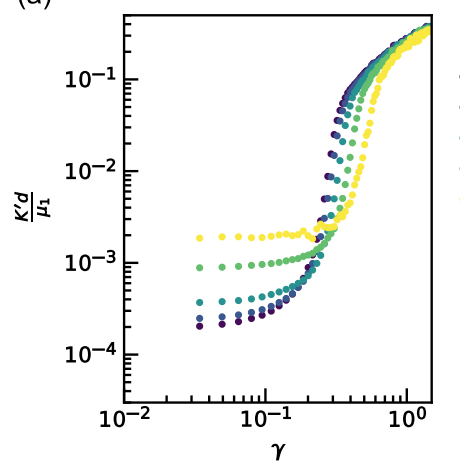

(c)

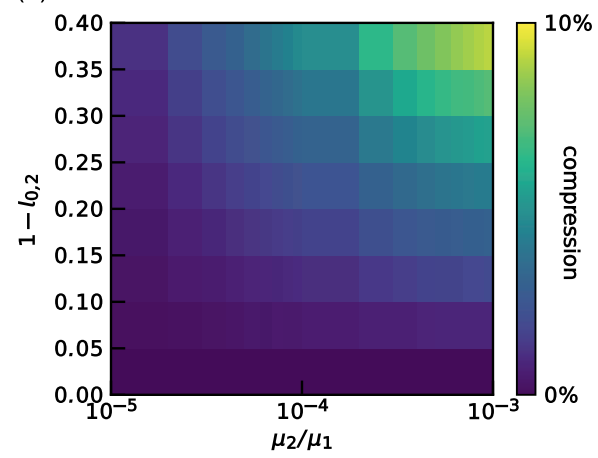

$\mu_{2} / \mu_{1} ; l_{0,2}$ (b)

- $1 \mathrm{e}-05 ; 1.00$

- $2 \mathrm{e}-05 ; 0.85$

- $5 \mathrm{e}-05 ; 0.75$

2e-04; 0.70

$5 e-04 ; 0.60$

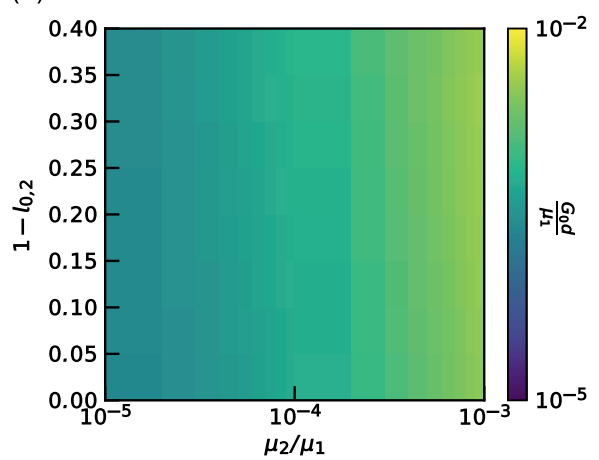

(d)

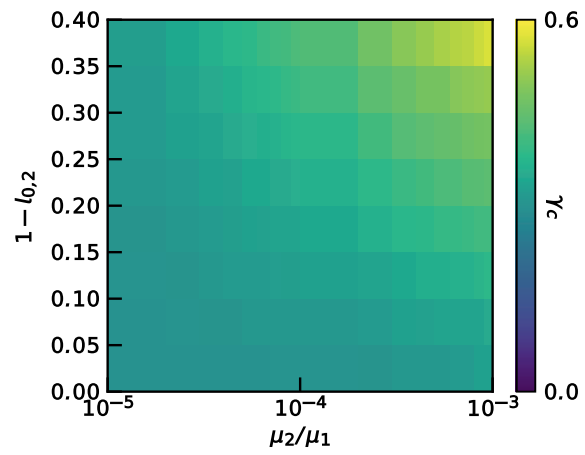

Figure 4.A.8. Mechanical properties of simulated composite networks with $\tilde{\kappa}=1 \times 10^{-4}$, corresponding to telocollagen. (a) Example strain-stiffening curves. (b) Linear modulus, (c) compression at mechanical equilibrium, (d) critical strain, shown in terms of the two matrix-related control parameters: matrix stiffness $\mu_{2}$ and rest length reduction $1-\ell_{0,2}$. 
(a)

(c)

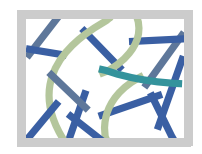

$E_{\min }>0$

Reset $\checkmark$ rest angles

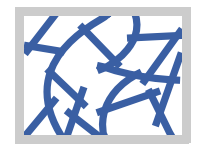

$E_{\min }=0$

(e)

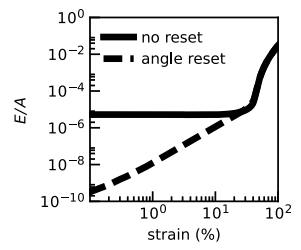

(b)

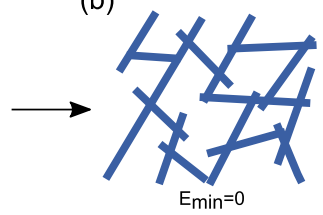

(d)

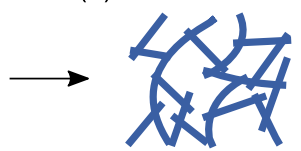

$\mathrm{E}_{\min }=0$

(f)

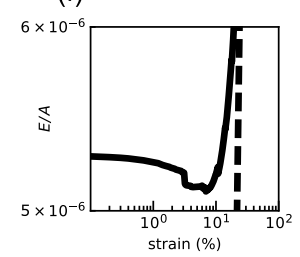

Figure 4.A.9. Establishing a mechanical balance in effective single-component network simulations by resetting the fibre angles. (a) If a network is compressed by forcing it to a smaller system size, the fibres will bend. This will increase the bending energy of some fibres, as indicated by the colour coding from blue (low energy) to yellow (high energy). (b) If the boundaries are removed, the system will go back to its original state in order to alleviate the excess bending energy. This is in contrast with the double network simulations, where a mechanical balance is established between the fibres and the matrix. (c) To prevent the system from moving back to its original state, we can reset the rest angles of the fibres in the compressed state. This also lowers the energy of the system, because now the fibres are in their rest state in this particular configuration. (d) Now, if the boundaries are removed, the system will retain its shape. (e) The necessity of the angle reset becomes clear if we compare the development of the total energy of the system, divided by the area as a function of shear $\left(\langle z\rangle=3.2, L=30, N_{\text {config }}=5\right)$. If the angles are not reset after compression, the energy starts at a high value, after which it goes down. If the angles are reset before shear, the behaviour is markedly different: the energy starts at zero and increases linearly up to the onset of strain stiffening. At the onset of strain stiffening, the curves with and without an angle reset converge, because here the stretching terms start to dominate. (f) Zoom-in of panel (e) showing that the total energy decreases upon increasing strain. 
(a)

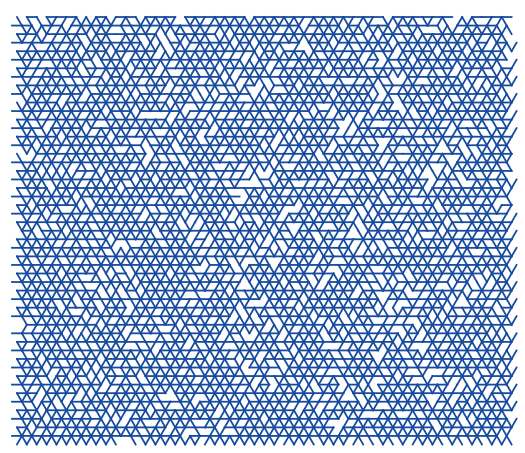

(c)
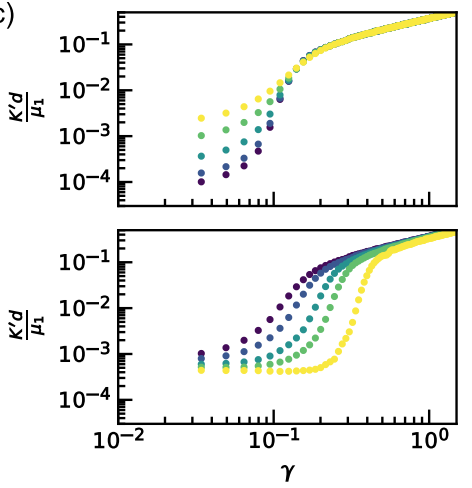

\section{- $0 \%$}

- $1 \%$

- $3 \%$

$5 \%$ (b)

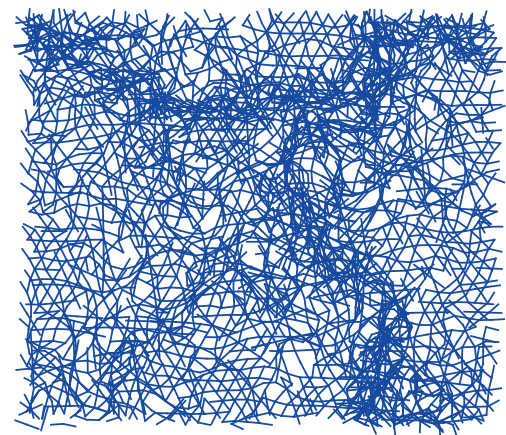

(d)

- $2 \mathrm{e}-05$

- 3e-05

- 7e-05

- 2e-04

5e-04

$10 \%$

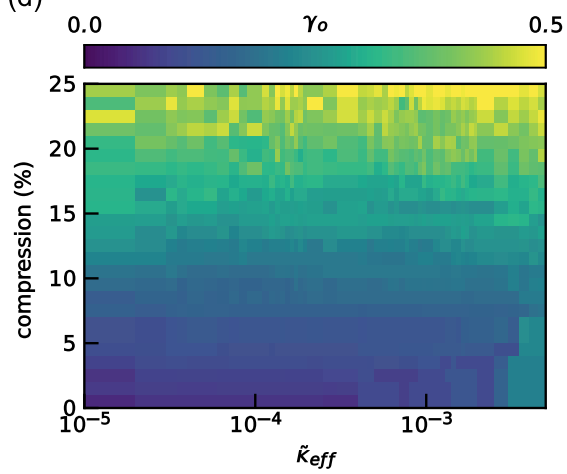

Figure 4.A.10. Mechanical response of an effective single-component model. Snapshots of a phantom network with $\langle z\rangle=3.2$ and $(L=50$ ) (a) before compression and (b) after $25 \%$ isotropic compression. (c) Modulus versus strain for different effective fibre rigidity $\tilde{\kappa}_{\text {eff }}$ (indicated in the legend) without compression (top panel), and modulus for fixed $\tilde{\kappa}_{\text {eff }}=1 \times 10^{-4}$ with different levels of compression (as indicated in the legend, bottom panel). (d) Map of the onset strain as a function of the bulk compressive strain and $\tilde{\kappa}_{\text {eff }}\left(N_{\text {config }}=5\right)$. 
(a)

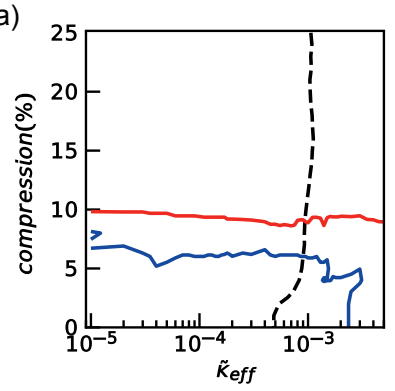

(d)

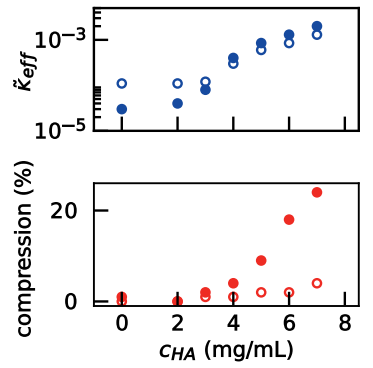

(b)

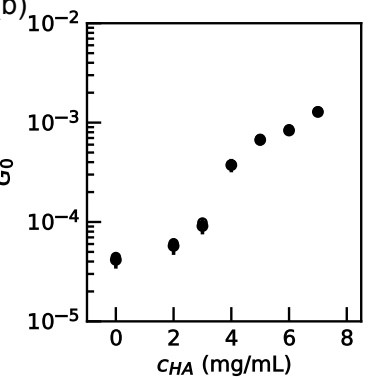

(e)

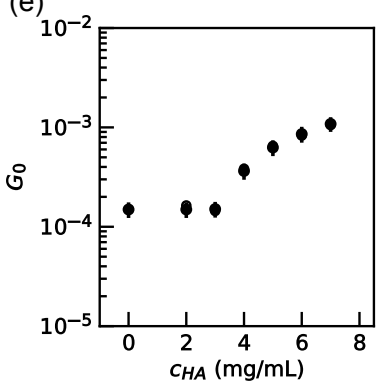

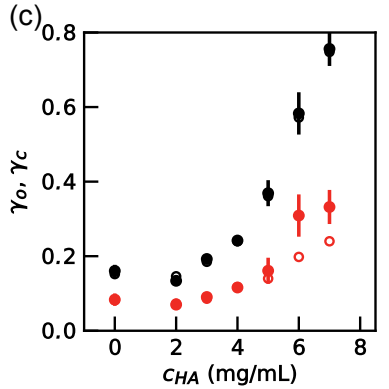

(f)

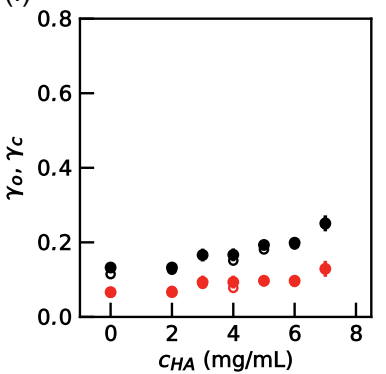

Figure 4.A.11. Mapping effective single-component network simulations on the experimental system. (a) Example of the mapping procedure. Using the simulation maps for the linear modulus, onset and critical strain (like Figure 4.A.10) we draw contour lines corresponding to the experimental values ( $1 \mathrm{mg} \mathrm{ml}^{-1}$ atelocollagen, $5 \mathrm{mg} \mathrm{ml}^{-1}$ hyaluronan) in the compression- $\tilde{\kappa}_{\text {eff }}$ plane. The dashed black line corresponds to the linear modulus, the red line to the critical strain and the blue line to onset strain. The point where the lines corresponding to the linear modulus and the critical strain cross identifies the simulation parameters that correspond to the experimental composite networks with given hyaluronan concentration. The onset strain is noisier than the linear modulus and the critical strain and is therefore not considered for the mapping. (b,c) Mapping of the linear modulus, onset strain (red) and critical strain (black) measured for atelocollagen composites on the simulation results. Solid symbols correspond to experimental values and open symbols to simulation values (often the two are overlapping). (d) Experimental results mapped on the simulation parameters of the effective single-component model for atelocollagen (solid symbols) and telocollagen (open symbols) as explained in Section 4.2.2. (e,f) Same as panels $(b, c)$ for telocollagen. To allow for mapping, the linear modulus found in the experiments was rescaled as described in Section 4.2.2. 


\section{List of symbols}

\begin{tabular}{|c|c|}
\hline Symbol & Description \\
\hline$A$ & Area covered by the two-dimensional lattice \\
\hline$c_{\mathrm{HA}}$ & Hyaluronan concentration \\
\hline$d$ & Lattice spacing \\
\hline E & Total elastic energy \\
\hline$E_{\text {stretch }}^{\text {fibre }}$ & Elastic energy stored in the system due to fibre stretching \\
\hline & Elastic energy stored in the system due to fibre bending \\
\hline$E_{\text {stretch }}^{\text {matrix }}$ & Elastic energy stored in the system due to matrix \\
\hline$f$ & Critical exponent related to strain stiffening behaviour \\
\hline$f_{\text {stretch, } \boldsymbol{x}_{i}}$ & $\begin{array}{l}\text { Stretching force exerted on node } i \text { by the fibre segment between } \\
\text { node } i \text { and node } j\end{array}$ \\
\hline$G_{0}$ & Linear elastic modulus \\
\hline$G_{\text {meas }}$ & Measured elastic modulus of the collagen-hyaluronan composite \\
\hline$G_{\text {sum }}$ & $\begin{array}{l}\text { Estimated elastic modulus of the collagen-hyaluronan composite } \\
\text { based on the sum of the modulus measured for the individual } \\
\text { components }\end{array}$ \\
\hline$K^{\prime}$ & Differential modulus defined as $\delta \sigma / \delta \gamma$ \\
\hline$\ell$ & Segment length, defined as the distance between nodes \\
\hline$\ell_{0,1}$ & Fibre segment rest length \\
\hline$\ell_{0,2}$ & Matrix segment rest length \\
\hline$\ell_{c}$ & Average fibre length in between crosslinks \\
\hline$L$ & Lattice size, with $L \times L$ the number of nodes \\
\hline$L_{0}$ & Length of a fibre \\
\hline$N_{\text {config }}$ & $\begin{array}{l}\text { Number of network realizations that are considered per data point } \\
\text { in the simulations }\end{array}$ \\
\hline $\boldsymbol{r}_{i j}$ & Vector describing the segment between two nodes $i$ and $j$ \\
\hline $\boldsymbol{x}_{i}$ & Coordinates of node $i$ \\
\hline$x_{m, n}$ & Coordinate of node $m$ on axis $n$ \\
\hline$z$ & Connectivity, number of bonds connected to a node \\
\hline$\langle z\rangle$ & Average connectivity over all nodes \\
\hline$\gamma$ & Shear strain \\
\hline$\gamma_{0}$ & Onset strain \\
\hline$\gamma_{c}$ & Critical strain \\
\hline$\Delta \gamma$ & Distance from the critical strain in terms of strain, $\gamma-\gamma_{c}$ \\
\hline
\end{tabular}


$\theta$

$\theta_{0}$

$\kappa$

$\tilde{\kappa}$

$\tilde{\kappa}_{\text {eff }}$

$\mu_{1}$

$\mu_{2}$

$\tilde{\mu}_{2}$

$\sigma$

$\sigma_{o}$

$\sigma_{\mathcal{C}}$

$\phi$
Angle between two neighbouring fibre segments

Rest angle between two neighbouring fibre segments

Bending rigidity, also written as $\kappa_{1}$ to indicate the bending rigidity of a fibre

Dimensionless or reduced bending rigidity

Effective reduced bending rigidity for a composite network

Stretching rigidity of a fibre segment

Stretching rigidity of a matrix segment

Dimensionless or reduced matrix stretching rigidity

Stress

Onset stress

Critical stress

Critical exponent related to strain stiffening behaviour 


\section{References}

[1] Y. Lanir, Multi-scale Structural Modeling of Soft Tissues Mechanics and Mechanobiology. Journal of Elasticity 129, 7-48 (2017).

[2] Y. C. Fung, Elasticity of soft tissues in simple elongation. The American journal of physiology 213, 1532-1544 (1967).

[3] M. S. Hall, F. Alisafaei, E. Ban, X. Feng, C. Y. Hui, V. B. Shenoy, M. Wu, Fibrous nonlinear elasticity enables positive Mechanical feedback between cells and ECMs. Proceedings of the National Academy of Sciences of the United States of America 113, 14043-14048 (2016).

[4] S. Van Helvert, C. Storm, P. Friedl, Mechanoreciprocity in cell migration. Nature Cell Biology 20, 8-20 (2018).

[5] C. Storm, J. J. Pastore, F. C. MacKintosh, T. C. Lubensky, P. A. Janmey, Nonlinear elasticity in biological gels. Nature 435, 191-194 (2005).

[6] S. Motte, L. J. Kaufman, Strain stiffening in collagen i networks. Biopolymers 99, 35-46 (2013).

[7] A. J. Licup, S. Münster, A. Sharma, M. Sheinman, L. M. Jawerth, B. Fabry, D. A. Weitz, F. C. MacKintosh, Stress controls the mechanics of collagen networks. Proceedings of the National Academy of Sciences of the United States of America 112, 9573-9578 (2015).

[8] A. Sharma, A. J. Licup, K. A. Jansen, R. Rens, M. Sheinman, G. H. Koenderink, F. C. Mackintosh, Strain-controlled criticality governs the nonlinear mechanics of fibre networks. Nature Physics 12, 584-587 (2016).

[9] D. F. Holmes, Y. Lu, T. Starborg, K. E. Kadler, Collagen Fibril Assembly and Function, vol. 130 (Elsevier Inc., 2018), first edn.

[10] C. P. Broedersz, F. C. Mackintosh, Modeling semiflexible polymer networks. Reviews of Modern Physics 86, 995-1036 (2014).

[11] J. C. Maxwell, On the calculation of the equilibrium and stiffness of frames. The London, Edinburgh, and Dublin Philosophical Magazine and Journal of Science 27, 294-299 (1864).

[12] P. R. Onck, T. Koeman, T. Van Dillen, E. Van Der Giessen, Alternative explanation of stiffening in cross-linked semiflexible networks. Physical Review Letters 95, 178102 (2005).

[13] J. K. Mouw, G. Ou, V. M. Weaver, Extracellular matrix assembly: A multiscale deconstruction. Nature Reviews Molecular Cell Biology 15, 771-785 (2014).

[14] J. M. Van Doorn, L. Lageschaar, J. Sprakel, J. Van Der Gucht, Criticality and mechanical enhancement in composite fiber networks. Physical Review E 95, 042503 (2017).

[15] A. S. Shahsavari, R. C. Picu, Exceptional stiffening in composite fiber networks. Physical Review E 92, 012401 (2015).

[16] V. K. Lai, D. S. Nedrelow, S. P. Lake, B. Kim, E. M. Weiss, R. T. Tranquillo, V. H. Barocas, Swelling of Collagen-Hyaluronic Acid Co-Gels: An In Vitro Residual Stress Model. Annals of Biomedical Engineering 44, 2984-2993 (2016). 
[17] D. A. Narmoneva, J. Y. Wang, L. A. Setton, Nonuniform swelling-induced residual strains in articular cartilage. Journal of Biomechanics 32, 401-408 (1999).

[18] A. J. Michalek, M. G. Gardner-Morse, J. C. Iatridis, Large residual strains are present in the intervertebral disc annulus fibrosus in the unloaded state. Journal of Biomechanics 45, 1227-1231 (2012).

[19] C. Voutouri, T. Stylianopoulos, Accumulation of mechanical forces in tumors is related to hyaluronan content and tissue stiffness. PLoS ONE 13, 1-15 (2018).

[20] F. Burla, J. Tauber, S. Dussi, J. van der Gucht, G. H. Koenderink, Stress management in composite biopolymer networks. Nature Physics 15, 549-553 (2019).

[21] C. P. Broedersz, K. E. Kasza, L. M. Jawerth, S. Münster, D. A. Weitz, F. C. MacKintosh, Measurement of nonlinear rheology of cross-linked biopolymer gels. Soft Matter 6, 4120-4127 (2010).

[22] K. A. Jansen, A. J. Licup, A. Sharma, R. Rens, F. C. MacKintosh, G. H. Koenderink, The Role of Network Architecture in Collagen Mechanics. Biophysical Journal 114, 2665-2678 (2018).

[23] C. P. Broedersz, X. Mao, T. C. Lubensky, F. C. Mackintosh, Criticality and isostaticity in fibre networks. Nature Physics 7, 983-988 (2011).

[24] S. B. Lindström, D. A. Vader, A. Kulachenko, D. A. Weitz, Biopolymer network geometries: Characterization, regeneration, and elastic properties. Physical Review E Statistical, Nonlinear, and Soft Matter Physics 82, 051905 (2010).

[25] E. Bitzek, P. Koskinen, F. Gähler, M. Moseler, P. Gumbsch, Structural relaxation made simple. Physical Review Letters 97, 170201 (2006).

[26] J. Feng, H. Levine, X. Mao, L. M. Sander, Nonlinear elasticity of disordered fiber networks. Soft Matter 12, 1419-1424 (2016).

[27] R. Rens, M. Vahabi, A. J. Licup, F. C. MacKintosh, A. Sharma, Nonlinear mechanics of athermal branched biopolymer networks. Journal of Physical Chemistry B 120, 5831-5841 (2016).

[28] A. Sharma, A. J. Licup, R. Rens, M. Vahabi, K. A. Jansen, G. H. Koenderink, F. C. MacKintosh, Strain-driven criticality underlies nonlinear mechanics of fibrous networks. Physical Review E 94, 042407 (2016).

[29] R. C. Picu, Mechanics of random fiber networks - A review. Soft Matter 7, 6768-6785 (2011).

[30] A. S. G. Van Oosten, M. Vahabi, A. J. Licup, A. Sharma, P. A. Galie, F. C. MacKintosh, P. A. Janmey, Uncoupling shear and uniaxial elastic moduli of semiflexible biopolymer networks: compression-softening and stretch-stiffening. Scientific Reports 6, 19270 (2016).

[31] A. J. Licup, A. Sharma, F. C. Mackintosh, Elastic regimes of subisostatic athermal fiber networks. Physical Review E 93, 41-44 (2016). 
[32] K. S. Rankin, D. Frankel, Hyaluronan in cancer-from the naked mole rat to nanoparticle therapy. Soft Matter 12, 3841-3848 (2016).

[33] J. P. Berezney, O. A. Saleh, Electrostatic Effects on the Conformation and Elasticity of Hyaluronic Acid, a Moderately Flexible Polyelectrolyte. Macromolecules 50, 1085-1089 (2017).

[34] M. Vahabi, A. Sharma, A. J. Licup, A. S. G. van Oosten, P. A. Galie, P. A. Janmey, F. C. MacKintosh, Elasticity of fibrous networks under uniaxial prestress. Soft Matter 12, 5050-5060 (2016).

[35] M. Shayegan, T. Altindal, E. Kiefl, N. R. Forde, Intact Telopeptides Enhance Interactions between Collagens. Biophysical Journal 111, 2404-2416 (2016).

[36] G. H. Koenderink, Z. Dogic, F. Nakamura, P. M. Bendix, F. C. MacKintosh, J. H. Hartwig, T. P. Stossel, D. A. Weitz, An active biopolymer network controlled by molecular motors. Proceedings of the National Academy of Sciences of the United States of America 106, 15192-15197 (2009).

[37] K. A. Jansen, R. G. Bacabac, I. K. Piechocka, G. H. Koenderink, Cells actively stiffen fibrin networks by generating contractile stress. Biophysical Journal 105, 2240-2251 (2013).

[38] D. Mizuno, C. Tardin, C. F. Schmidt, F. C. MacKintosh, Nonequilibrium mechanics of active cytoskeletal networks. Science 315, 370-373 (2007).

[39] D. J. Cosgrove, Growth of the plant cell wall. Nature Reviews Molecular Cell Biology 6, 850-861 (2005).

[40] M. Jaspers, S. L. Vaessen, P. Van Schayik, D. Voerman, A. E. Rowan, P. H. Kouwer, Nonlinear mechanics of hybrid polymer networks that mimic the complex mechanical environment of cells. Nature Communications 8, 15478 (2017). 



\section{Chapter 5}

\section{Microscopic insights into the fracture of double elastic networks}

The toughness of a polymer material can increase significantly if two networks are combined into one material. This toughening effect is a consequence of a transition from a brittle to a ductile fracture response. Although this transition and the accompanying toughening effect have been demonstrated in hydrogels first, the concept has been proven effective in elastomers and in macroscopic composites as well. This suggests that the transition is not caused by a specific molecular architecture, but rather by a general physical principle related to the mechanical interplay between two interpenetrating networks. Here we employ theory and computer simulations, inspired by this general principle, to investigate how disorder controls the brittle-to-ductile transition both at the macroscopic and the microscopic level. A random spring network model featuring two different spring types, enables us to study the joined effect of initial disorder and network-induced stress heterogeneity on this transition. We reveal that a mechanical force balance gives a good description of the brittle-to-ductile transition. In addition, the inclusion of disorder in the spring model predicts four different fracture regimes along the brittle-to-ductile response in agreement with experimental findings. Finally, we show that the network structure can result in stress concentration, diffuse damage and loss of percolation depending on the fracture regime. This work thus provides a framework for the design and optimization of double network materials and underlines the importance of network structure in the toughness of polymer materials.

\section{Justin Tauber, Simone Dussi \& Jasper van der Gucht}

"Microscopic insights into the failure of double elastic networks"

Physical Review Materials 4, 063693 (2020) 


\subsection{Introduction}

Polymer networks, such as rubbers and gels, can undergo large deformation without losing elasticity. This property makes them ideal for numerous applications, for example in the biomedical field or in soft robotics. One of the factors that limits their applicability, however, is the brittleness of many polymer networks, which leads to inferior mechanical performance, such as low fracture toughness and strength ${ }^{1}$. In recent years, several strategies have been developed to toughen polymer networks by introducing dissipation mechanisms that delay crack propagation. One of the most successful strategies relies on the combination of two different polymer networks into one material, to create a so-called double network. In particular, extremely tough double network hydrogels have been produced by interpenetrating a stiff and weak first network with a soft and extensible second network ${ }^{2-11}$ (Figure 5.1(a)). Later, this toughening strategy was also shown to be effective for multi-network elastomers ${ }^{12,13}$ and macroscopic composites ${ }^{14,15}$ (Figure 5.1(b)). This suggests that the underlying principle that leads to toughening is not strongly dependent on molecular details, but governed more generally by an interplay between two mechanically different networks.

The increase in toughness of double networks is related to a transition from brittle to ductile fracture. This brittle-to-ductile transition (BDT) becomes apparent when looking at the stress-strain response of double networks subjected to tensile deformation, as schematically shown in Figure 5.1(c). In contrast to single polymer networks that typically fail in a brittle fashion (blue curve), tough double networks fracture in a ductile manner with significant softening and plastic deformation (leading to 'necking') prior to failure (yellow curve) ${ }^{13,16}$. This macroscopic softening is believed to be due to the progressive breaking of bonds in the stiff network (which is also called the sacrificial network), while the soft matrix formed by the second network stays intact. To obtain tough materials, it is thus crucial that the rupture of the first network occurs without leading to macroscopic cracks in the material.

Several theoretical models have been proposed to explain the toughness of double networks. The first of these are due to Tanaka ${ }^{17}$ and Brown ${ }^{18}$ that describe fracture around a defect, such as a notch or a crack. They assumed that a crack tip in the material is surrounded by a region in which the first network breaks by forming microcracks that are stabilized by bridging chains from the second network. Later extensions of these models explicitly accounted for the evolution of damage in the material and were able to describe the hysteresis in the stress-strain response, the progressive softening and the Mullins effect ${ }^{19-24}$. These phenomenological models assume that the stress field in the material can be described by continuum 
mechanics, which treats the material as a homogeneous elastic solid. However, it is known that double networks contain large heterogeneities, which play an important role in determining their mechanics ${ }^{25}$. To understand how microcracks in the material nucleate and propagate and to find a criterion that predicts the onset of macroscopic fracture, a model is needed that takes these heterogeneities into account.

One way to go forward is to include molecular details, for example using molecular dynamics simulations ${ }^{26-28}$. However, given the computational costs it is difficult to do this for large system sizes and to explore a large range of parameters. Alternatively, statistical models, such as the fibre bundle model, can give insight into the role of disorder on network fracture ${ }^{29,30}$. In such models, however, a priori assumptions must be made about the (re)distribution of stress among the

(a)

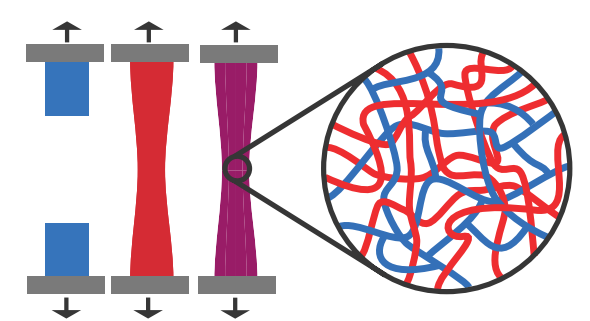

(b)

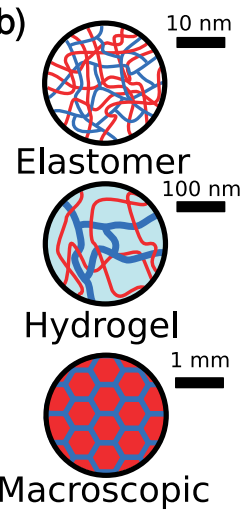

(c)

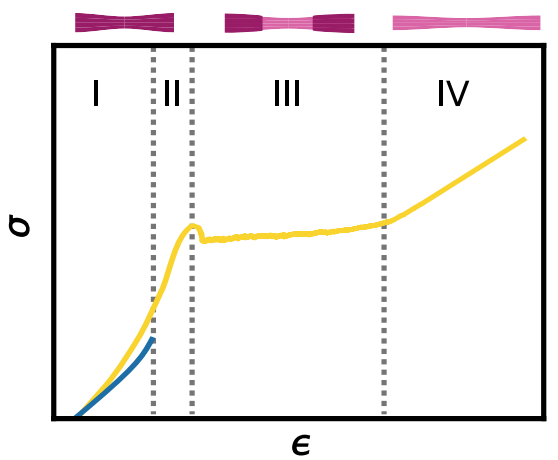

Figure 5.1. Double networks across the scale. (a) By combining a sacrificial network that is stiff and weak (blue), with a matrix network that is soft and strong (red), a (molecular) composite both stiff and strong (tough) can be created. (b) Typical double networks are made of elastomers ${ }^{12}$ and hydrogels ${ }^{2}$, but they can also be made in the form of macroscopic composites ${ }^{14}$. (c) Schematic of a brittle (blue) and a ductile (yellow) stress-strain response. 
different elements, so that they cannot explain the spatial evolution of damage in the materials and its relation to the structure of the networks.

Here, we develop a random double spring network model, which explicitly takes into account disorder and the resulting heterogeneous stress distributions. Rather than making assumptions about the distribution of strain and stress in the material, this distribution emerges naturally from the condition of mechanical equilibrium between the different networks. Because a network is included explicitly, the model provides information on the fracture process both at the macroscopic and the microscopic level. We demonstrate how this model predicts a simple criterion for the BDT, and we show how the nature of this transition is influenced by disorder and the resulting stress localization. We also compare our network model to a simple 1D multi-spring model, which can be solved analytically.

\subsection{Models and Methods}

The BDT is the universal mechanical feature of double network materials. In a brittle material, a small microcrack in the sacrificial network directly leads to global failure. On the contrary, a ductile material remains intact when microcracks develop. To describe this transition, we first present a simple 1D multi-spring model, which is an extension of a previously used two-spring model ${ }^{24,31}$ and which can be solved analytically (Figure 5.2(a)). While heterogeneity can be included in this 1D model, it does not take into account the network structure of the material and, therefore, cannot give an accurate description of the stress distribution in the material. We, therefore, consider a more realistic double random spring model that does allow for a heterogeneous stress distribution in the material (Figure 5.2(b)).

\subsubsection{Multi-spring model}

In the multi-spring model (MS), the double network is modelled as a series of elements, each consisting of two parallel springs, one representing the sacrificial network (with spring constant $\mu_{S}$ ) and one representing the matrix (with spring constant $\mu_{M}$ ) (Figure 5.2(a)). Each spring is assumed to be a linear Hookean spring, so that the force acting on it is given by $F_{i}=\mu_{i} \Delta \ell_{i}$, with $\Delta \ell_{i}$ the extension of spring $i$. Removal of a sacrificial spring in an element leads to the formation of a microcrack, which is bridged by the remaining matrix spring. As the elements are connected in series, the load on each element is equal. This force balance between the elements implies 
(a)

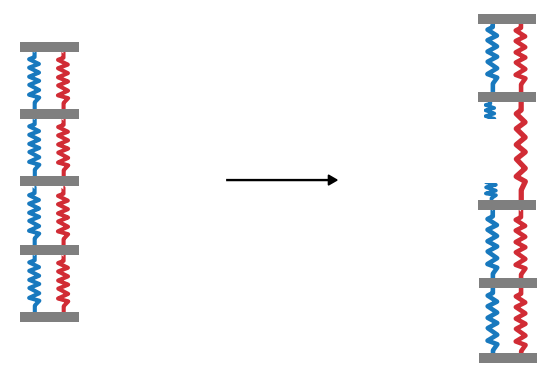

(b)

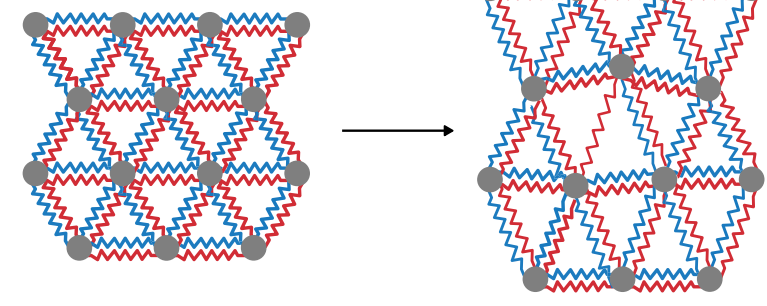

Figure 5.2. Minimal models for elastic double networks and their reaction to bond rupture. The springs represent elements of the sacrificial network (blue) and matrix network (red). (a) Multisprings (MS) model. (b) Random Spring Network (RSN) model. Simulations are performed for networks of $50 \times 50$ nodes.

$$
\sigma=\left(\mu_{M}+\mu_{S}\right) \Delta \ell_{D}=\mu_{M} \Delta \ell_{M},
$$

where $\Delta \ell_{D}$ and $\Delta \ell_{M}$ represent the extension of the network in the intact double network and in the matrix spanning the microcrack, respectively, and where $\sigma$ denotes the stress. The overall strain of the system $\varepsilon$ can be written as

$$
\varepsilon=\Delta \ell_{D}(1-\phi)+\Delta \ell_{M} \phi,
$$

where $\phi$ denotes the fraction of broken sacrificial bonds (i.e. the fraction of microcracks).

Rupture of a bond is assumed to occur instantaneously when the extension of a spring $\Delta \ell_{i}$ exceeds its threshold $\lambda_{i}$. In the absence of disorder, all sacrificial bonds have the same threshold $\lambda_{S}$ and all matrix bonds have the same threshold $\lambda_{M}$. Brittle fracture after formation of a microcrack occurs when the bridging matrix bond reaches its threshold before the other sacrificial bonds, while ductile fracture occurs when the sacrificial bonds reach their threshold first. From 
Equation 5.1 it immediately follows that the brittle-to-ductile transition occurs when $\left(\mu_{S}+\mu_{M}\right) \lambda_{S}=\mu_{M} \lambda_{M}$. We can, therefore, predict which system parameters are required for a system at the BDT. For example, the sacrificial bond threshold $\lambda_{S}^{*}$ for which the rupture force of a (matrix-reinforced) sacrificial bond is equal to the rupture force of a matrix bond is given by

$$
\lambda_{S}^{*}=\frac{\mu_{M} \lambda_{M}}{\mu_{S}+\mu_{M}} .
$$

More generally, we can define a parameter $\Delta \alpha$ to quantify the distance from the BDT:

$$
\Delta \alpha=\frac{\mu_{M} \lambda_{M}}{\left(\mu_{M}+\mu_{S}\right) \lambda_{S}}-1 .
$$

If $\Delta \alpha=0$ the system is at the BDT. Brittle fracture occurs for $\Delta \alpha<0$; in this case only one microcrack is enough to cause global failure so the fraction of broken sacrificial bonds at failure is $\phi_{f}=1 / N$ with $N$ the system size. Ductile fracture occurs for $\Delta \alpha>0$, where all sacrificial bonds break before the system fails globally, so $\phi_{f}=1$. It follows from this analysis that for ductile fracture we need $\lambda_{S}<\lambda_{M}$, which explains why the creation of tough, ductile networks requires the sacrificial network to be much weaker than the matrix.

For a perfectly homogeneous system, the BDT in this model is an abrupt transition. Experiments, however, show that the descriptors of fracture vary in a continuous manner through the BDT ${ }^{16}$. We, therefore, introduce disorder by assuming that the thresholds of the sacrificial network vary according to a certain distribution $P\left(\lambda_{S}\right)$. All other parameters are taken the same. In this case, the sacrificial bonds will fail progressively, with the weakest bonds breaking first and the stronger bonds breaking later. At a given strain $\varepsilon$, all sacrificial bonds for which $\lambda_{S}<\Delta \ell_{D}$ are broken. In the continuous limit (for $N \gg 1$ ), the fraction of broken sacrificial bonds can thus be written as

$$
\phi(\varepsilon)=\int_{0}^{\Delta \ell_{D}} P\left(\lambda_{S}\right) \mathrm{d} \lambda_{D}
$$

Here, we will consider a Gaussian threshold distribution with mean $\left\langle\lambda_{S}\right\rangle$ and standard deviation $\delta \lambda$. For this case, it follows from Equations 5.1 and 5.5 that at the moment of global failure (where $\Delta \ell_{M}=\lambda_{M}$ ), the fraction of broken sacrificial bonds is given by

$$
\phi_{f}=\frac{1}{2}[1+\operatorname{erf}(\Delta \tilde{\alpha})]
$$


with

$$
\Delta \tilde{\alpha}=\frac{\Delta \alpha\left\langle\lambda_{S}\right\rangle}{\delta \lambda \sqrt{2}}
$$

a normalized parameter to quantify the distance to the BDT, which takes into account the disorder in the thresholds. The fraction of broken sacrificial bonds thus increases gradually from 0 to 1 along the BDT and the parameter $\Delta \tilde{\alpha}$ sets the steepness of this transition. With increasing disorder, the transition becomes more gradual. The strain at break follows from Equations 5.1 and 5.2:

$$
\varepsilon_{f}=\lambda_{M}\left(\frac{\mu_{M}+\phi_{f} \mu_{S}}{\mu_{M}+\mu_{S}}\right) .
$$

In the brittle regime $\left(\Delta \tilde{\alpha} \ll-1, \phi_{f} \approx 0\right)$ we find $\varepsilon_{f}=\mu_{M} \lambda_{M} /\left(\mu_{M}+\mu_{S}\right)$, while in the ductile regime $\left(\Delta \tilde{\alpha} \gg 1, \phi_{f} \approx 1\right)$ we have $\varepsilon_{f}=\lambda_{M}$.

\subsubsection{Random Spring Network Model}

Being a 1D model, the multi-spring model cannot account for stress heterogeneity and the resulting localization of stresses. We, therefore, consider a random spring network model (RSN) as shown in Figure 5.2(b) composed of $L \times L$ nodes arranged on a triangular lattice whose nearest neighbours are connected by the same element we introduced for the multi-spring model ( $L=50$ for all RSN simulations). Now, upon applying an external load, stress concentration is possible due to the topological restrictions imposed by the network structure.

Following the experimentally found guidelines for making tough double networks ${ }^{2}$ we consider materials in which the sacrificial network is stiff and weak, while the matrix is soft and strong. This means that the elastic constant of sacrificial springs $\mu_{S}$ is always higher than the one of the matrix springs $\mu_{M}$. We, therefore, vary the ratio of the stiffnesses such that $0<\mu_{M} / \mu_{S}<1$. To reflect the asymmetry in network strengths, we fix $\lambda_{M}=4.0$ and vary $\lambda_{S}$ such that $\lambda_{S}<\lambda_{M}$. In all cases, we keep the parameters of all matrix springs the same. To implement disorder at the bond-level, the rupture thresholds $\lambda_{S}$ are picked from a Gaussian distribution as in the MS model. Nodes at the bottom and top are fixed in the $y$-direction, but they can slide along the $x$-direction. Along the $x$-axis periodic boundary conditions are implemented.

We characterize the mechanical response of the network by applying an extensional strain $\varepsilon$ along the $y$-axis in small steps of $0.1 \%$ strain. We consider quasistatic loading and assume that the networks remain in mechanical equilibrium at each step, i.e. it settles in its minimum energy state. Therefore, after every step, 
the total energy of the network is minimized by displacing the nodes using the FIRE algorithm ${ }^{32}$ with a tolerance of $F_{\mathrm{rms}} \leq 1 \times 10^{-5}$. Here, $F_{\mathrm{rms}}$ is the maximum root mean squared force allowed in the system ${ }^{32}$. To simulate fracture, we break all bonds which exceed their rupture threshold $\lambda_{i}$ consecutively. After the rupture of every single bond the energy of the system is minimized. Once all overstretched bonds are broken, the next strain step is determined according to the bond that is closest to its rupture threshold with a minimum step size of $0.001 \%$ strain.

For every strain in the simulations the virial stress is calculated from the forces exerted by the springs on the nodes. As the system is elongated along the $y$-direction, we consider the $y y$-component of the virial tensor as a measure for the stress $\sigma$. We define the softening strain $\varepsilon_{\text {soft }}$ as the strain where the stiffness drops below the initial stiffness, and the failure strain $\varepsilon_{f}$ and failure stress $\sigma_{f}$ as the strain and stress for which the system becomes disconnected along the y-direction. For every data point 50 simulations are performed, error bars represent standard deviation.

\subsubsection{Classification of stress-strain responses}

We use the following procedure to classify the stress-strain response of the different networks. We define four categories: brittle (B), quasibrittle (QB) and two ductile phases (D1 and D2). The characterization is based on the stiffness of the network during the deformation, defined as the derivative of the stress-strain response, normalized by the initial stiffness. Examples are shown in Figure 5.A.3. If a material is brittle (B), its stiffness during extension will stay constant, or at least will not drop far below its initial stiffness. In case of quasibrittle (QB) fracture, softening occurs, which causes a sharp drop in material stiffness. We identify the softening strain as the point where the ratio between the stiffness and the linear stiffness $E(\varepsilon) / E_{0}$ drops below 1.0, the initial stiffness. A material that shows ductile fracture also goes through this softening stage, but after softening a plateau can be seen in the stiffness curve. The transition from QB to D1 is based on the existence of the plateau. The plateau is identified as the strain region where $E(\varepsilon) / E_{0} \leq E_{\min } / E_{0}+0.05$, provided $E_{\min } / E_{0}<0.7$. This region must span at least $50 \%$ of strain in order to be identified as a plateau, otherwise we classify the fracture response as QB. If the material breaks in this plateau region, it is classified as D1. If instead the material response exceeds this plateau and achieves a higher stiffness, we classify this response as D2. We note that in our case the final stiffness is always lower than the initial stiffness, since the last part of the D2 response is controlled by the matrix elasticity for which we have $\mu_{M} / \mu_{S}<1.0$. This final strain 
region is identified as the region from the end of the plateau until global failure. The stiffness in this plateau must exceed $E_{0}$ by $15 \%$ in order to be identified as D2.

For the MS model the stiffness is determined as the linear piece-wise derivative of the stress-strain curve. For the RSN model, the stress-strain response is smoothed before determining the fracture response in order to reduce the influence of small fluctuations due to the rupture of bonds. The smoothing procedure has 4 steps. Firstly, only strain points are selected that have a higher stress than the previous points. Secondly, we make the data series equispaced in strain via linear interpolation with an interval of $0.1 \%$ strain. Thirdly, we smooth the curve via a linear fit over a window of $2 \%$ strain, the resulting stress-strain curve has a strain interval of $1 \%$ strain. Finally, we determine the stiffness (first derivative of the stress-strain curve) using a Savitsky-Golay algorithm $\left(4^{\text {th }}\right.$ order and $41 \%$ strain window). At the edges half of the window size is fitted with a $4^{\text {th }}$ order polynomial.

\subsubsection{Crack detection algorithm}

To monitor the microscopic fracture patterns in the RSN model, we keep track of the formation and development of cracks in the sacrificial network. For simplicity, in this analysis we only consider sacrificial bonds that align with the strain field and, therefore, carry most of the stress (in our case, these are the non-horizontal initial bonds). At the beginning of the simulation, the sacrificial network is intact but as soon as bonds break voids appear. These voids are defined as cracks. By studying the number of voids and their size in terms of the number of broken sacrificial bonds, we obtain a complete picture of the microscopic fracture behaviour. In particular, we calculate the size of the largest crack with respect to the total number of sacrificial bonds in the undeformed network and express this as the fraction $\phi_{\text {large }}$. All the broken sacrificial bonds that do not end up in the largest crack represent the non-localized damage occurring throughout the network. We define $\phi_{\text {diff }}=\phi-\phi_{\text {large }}$ to quantify the diffuse damage.

\subsection{Results \& discussion}

\subsubsection{Fracture regimes of double networks}

The aim of this chapter is to systematically explore the effect of disorder and stress heterogeneity on the fracture of double networks, focusing especially on the role of the network. To do this in a systematic fashion, we will extensively compare the results of the random spring network (RSN) model with the 1D multi-spring (MS) model lacking any network structure. 


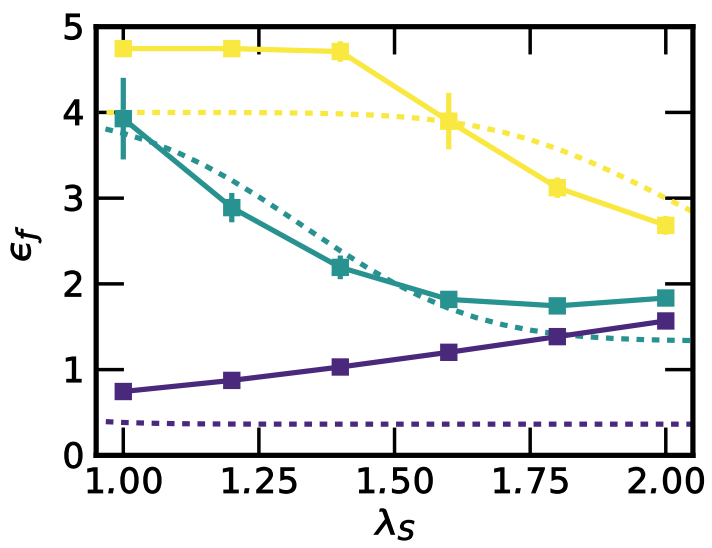

Figure 5.3. Failure strain, $\varepsilon_{f}$, versus the average rupture threshold $\left\langle\lambda_{S}\right\rangle$ in systems with $\delta \lambda=$ 0.250. Results are shown for the RSN model (solid lines) and the MS model (dashed lines) for stiffness ratios $\mu_{M} / \mu_{S}=0.10$ (purple), 0.50 (green) and 1.00 (yellow)).

We start our comparison by checking a prediction from the MS model for the dependence of the failure strain $\varepsilon_{f}$, described in Equation 5.8, on the sacrificial bond properties $\lambda_{S}$ and $\mu_{M} / \mu_{S}$ in the presence of disorder (Figure 5.3). Although it is clear that both the MS model and the RSN model follow the same trend, the overall trend is not immediately obvious. Intuitively, it is expected that the failure strain should increase with an increase in $\lambda_{S}$. However, only at the lowest stiffness ratio, we see that the $\varepsilon_{f}$ increases with $\lambda_{S}$. At high stiffness ratios, this trend is reversed. This transition is a consequence of the brittle-to-ductile transition (BDT). From Equation 5.4 it can be deduced that an increase in $\lambda_{S}$ brings the system from the ductile into the brittle regime. The transition is not observed at the lowest stiffness ratio, because all those systems are already in the brittle regime. Similar trends can be found for the material strength and the work of extension (Figure 5.A.1).

These results show that we can tune the fracture response based on the properties of the individual sacrificial bonds. Taking a broader view at the entire stress-strain response of the RSN, we can differentiate between four distinct responses, shown in Figure 5.4(a). There are two brittle responses, brittle (B) and quasibrittle $(\mathrm{QB})$, that can be distinguished by the (slight) softening that occurs before global failure in the QB case. There are also two ductile responses (D1 and D2) that both show a clear plateau in the stress-strain response after softening. In case of D1, global failure occurs within this plateau. For D2, a second increase in 
(a)

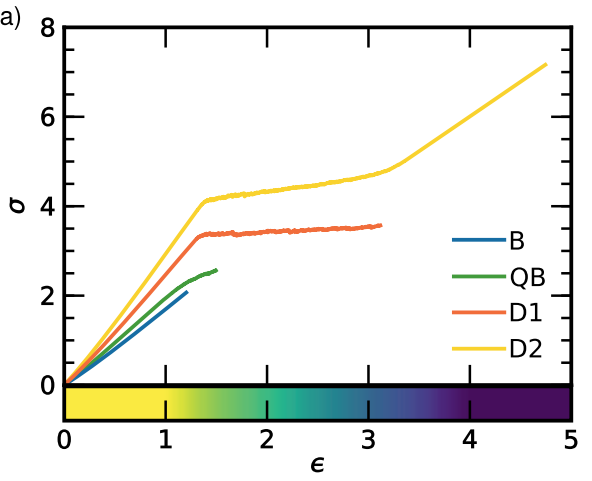

(b)

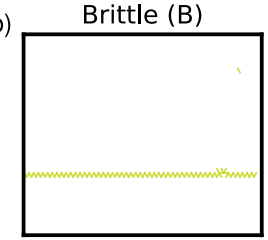

(d)

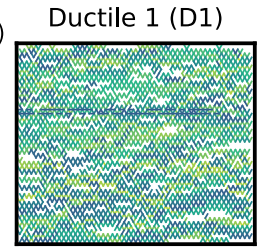

(c)

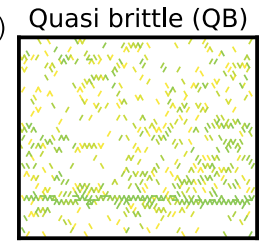

(e)

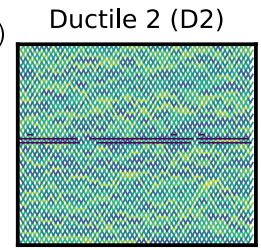

Figure 5.4. Typical fracture response of double networks obtained via the RSN model. (a) The four distinct stress-strain responses: brittle (B), quasibrittle (QB), the first ductile regime (D1), and the second ductile regime (D2). (b)-(e) Corresponding fracture patterns. Every line represents a broken sacrificial bond, colour coded according to the strain at which it failed (see colour bar above).

the stress precedes global failure. These same four responses are also found in the MS model (Figure 5.A.3).

By contrast, at the microscopic level the fracture behaviour of the RSN is distinct from the MS model. In Figure 5.4(b-e), we plot the broken bonds of the sacrificial network colour coded according to the strain at which they break. We clearly observe that the number of broken sacrificial bonds increases from B to D2 type of fracture. In B, all broken bonds are part of a single crack at the location where global failure occurs. A similar pattern is observed for QB, where, however, more bonds fail homogeneously throughout the sample before the final crack appears. It is the rupture of these bonds that causes the softening observed in the QB stress-strain response. The ductile regimes are characterized by many bonds breaking simultaneously. Such avalanches occur over a large strain range, from the moment of softening until global failure. In case of D2 fracture, all the non-horizontal bonds of the sacrificial network, which are the elastically active bonds, are broken. In the MS model we can not differentiate between fracture patterns, because the load is homogeneous throughout the system, so that stress can not concentrate and damage will always be homogeneous.

We note that both the RSN model and the MS model capture the four different mechanical responses observed in experiments on elastomers ${ }^{13}$, hydrogels ${ }^{2}$ and macroscopic composites ${ }^{14,15}$. The clear difference in fraction of broken sacrificial bonds between (quasi)brittle and ductile responses is also well established experi- 

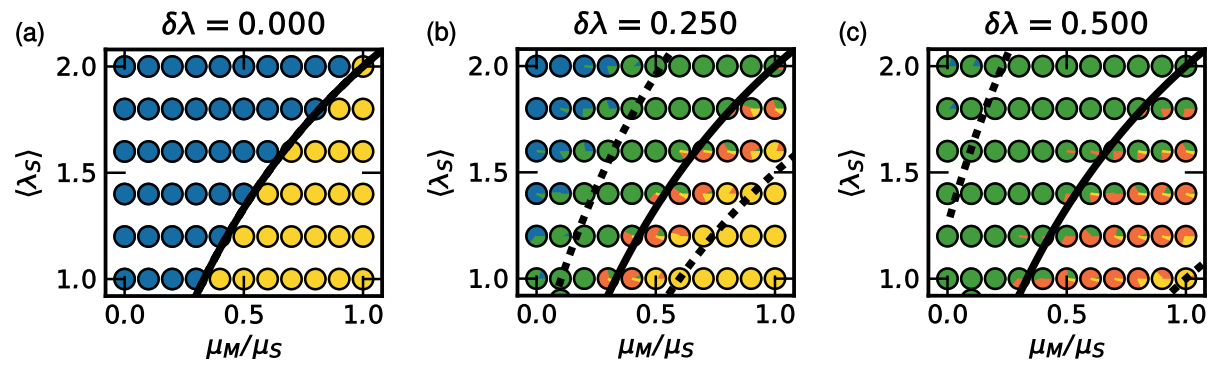

Figure 5.5. Macroscopic mechanical phase diagrams in the $\left(\mu_{M} / \mu_{S}, \lambda_{S}\right)$-plane. Each panel corresponds to a different value of disorder $\delta \lambda$. Symbols are pie-charts colour coded according to the frequency of the four fracture regimes (B (blue), QB (green), D1 (orange), D2 (yellow)) observed from 50 independent simulations. The black line indicates the BDT according to the MS model. The dashed lines indicate the B-to-QB transition $(\Delta \tilde{\alpha}=-2.5)$ and the D1-to-D2 transition $(\Delta \tilde{\alpha}=2.0)$ in the MS model (Figure 5.A.4).

mentally ${ }^{13,16}$. Furthermore, the softening response, which is irreversible both in our model and in the experiments, is attributed to early damage to bonds in the sacrificial network ${ }^{22}$ which has been shown to occur homogeneously throughout the network well before global failure ${ }^{13}$. Finally, also a plateau in the stress response is typically observed in experiments after the yielding of the material, often accompanied by a necking region where only sacrificial bonds break ${ }^{13}$. We conclude that both the RSN and MS model represent a minimal yet insightful model to study fracture in elastic double networks both at the macroscopic and microscopic level and, therefore, we can proceed to a more detailed analysis.

\subsubsection{Macroscopic characterization of the fracture regimes}

The results in Figure 5.3 clearly demonstrate the dependence of the fracture response on the properties of the sacrificial bonds: $\left\langle\lambda_{S}\right\rangle$, and $\mu_{M} / \mu_{S}$. To study the joined effect of these properties together with the disorder in the rupture threshold $(\delta \lambda)$, we construct phase diagrams of the fracture response (Figure 5.5) based on the four different regimes described above (see the Section 5.2.3 for details). For each point in the diagram we use a pie-chart symbol indicating the frequency of the four different regimes observed over 50 independent simulations. We first focus on the case with no disorder $\delta \lambda=0$, shown in Figure 5.5(a), and observe that both $\mu_{M} / \mu_{S}$ and $\lambda_{S}$ control the sharp transition from the BDT. Also, the BDT of the RSN model coincides with the BDT of the MS model (Equation 5.4) as indicated by the black line. 
Next, we consider the cases with disorder, plotted in Figure 5.5 (b) and (c), and observe the appearance of the intermediate regimes QB and D1. These two regimes appear around the BDT and upon increasing disorder $\delta \lambda$ progressively span the entire explored parameter space, which is a clear indication that disorder controls the position of these transitions. In addition, the transitions between the regimes are less sharp when the disorder is larger, as testified by the less homogeneously coloured pie-chart symbols. Indeed, the mechanical responses of networks with large $\delta \lambda$ depend on the exact realization of the network (exact distribution of threshold values and their spatial organization), even when having the same $\mu_{M} / \mu_{S}$ and $\left\langle\lambda_{S}\right\rangle$, especially around boundaries between different regimes. A similar dependence of the location of the B-to-QB and D1-to-D2 transitions on disorder is also predicted by the MS model (dashed lines, see Figure 5.A.4 for further information). At low disorder, the location of the boundaries corresponds well between the models, but at high disorder the boundaries move away from each other. This comparison reveals that the type of load sharing (equal load sharing in the MS model or network controlled load sharing in the RSN model) is not essential for the occurrence of any of the four regimes. Nevertheless, the stress redistribution via the network does affect how far from the BDT these regimes occur. Furthermore, disorder clearly plays an important role also in the experimental systems since QB and D1 responses are typically observed experimentally, and we show that these occur only in presence of disorder. Our results confirm that knowing the average value of the bond strengths might not be enough to predict the fracture regime, in agreement with recent phenomenological models aiming to capture softening and the Mullins effect in double networks ${ }^{22}$.

\subsubsection{Fracture at the microscopic level}

In the previous section, we found that the macroscopic force balance reasonably captures the transition from (quasi)brittle to ductile fracture in the RSN model and that disorder influences this transition. Furthermore, we have seen in Figure 5.4(b)(e) that as long as some disorder is present distinct fracture patterns develop in the RSN that suggest a tight link between the macroscopic and microscopic fracture process. In this section, we explore several indicators of microscopic fracture in disordered RSN systems, with a special focus on stress heterogeneity, and we consider to what extent the force balance influences the microscopic fracture processes in double networks. 
(a)

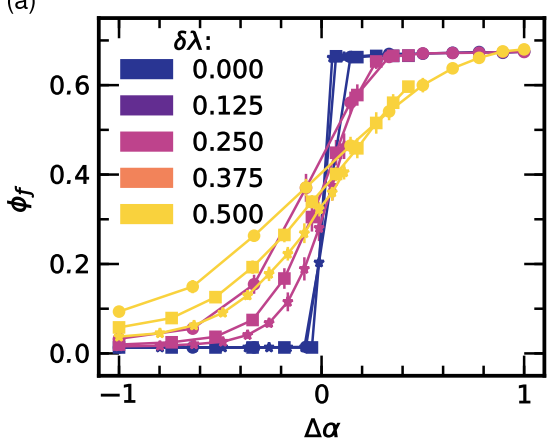

(b)

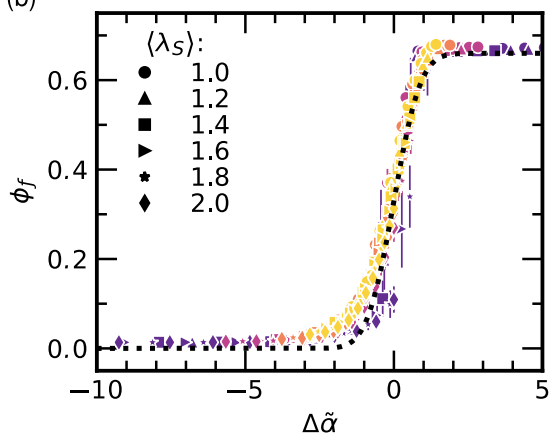

Figure 5.6. Rescaling of the fraction of broken bonds $\phi_{f}$. (a) The fraction of total broken bonds $\phi_{f}$ obtained in simulations of RSN is plotted against $\Delta \alpha$, which quantifies the distance from the BDT predicted by the equal load sharing models. (b) $\phi_{f}$ from RSN versus $\Delta \tilde{\alpha}$. The dashed black line indicates the theoretical prediction for $\phi_{f}$ according to the MS model (Equation 5.4). In the RSN model only the vertically aligned bonds are expected to break; therefore, the prediction of the MS model is multiplied by a factor $2 / 3$. The symbols correspond to the $\lambda_{S}$ value (see legend panel (b)) colour coded according to the amount of disorder $\delta \lambda$ (see legend panel (a)). Error bars indicate standard deviation based on 50 configurations.

\section{The fraction of broken bonds}

The first parameter we explore is the fraction of broken sacrificial bonds at global failure $\phi_{f}$, as we have a direct prediction from the MS model. In Figure 5.6(a), we plot $\phi_{f}$ as a function of $\Delta \alpha$, the distance from the BDT as introduced in Equation 5.4 for various amounts of disorder $\delta \lambda$. Clearly, we see a strong increase in the number of broken bonds upon going from the brittle to the ductile regime. For $\delta \lambda=0$ we observe that all data points collapse on a step function exactly at the $\mathrm{BDT}, \Delta \alpha=0$. When disorder is introduced, the transition becomes more gradual, corresponding to the shift of the B-to-QB and D1-to-D2 transition in Figure 5.5. We can understand these effects by pointing out that both weak and strong bonds are introduced due to the spread in sacrificial bond strength. For $\Delta \alpha<0, \phi_{f}$ is increased by the disorder due to the presence of weak springs that break before brittle failure occurs. Similarly, for $\Delta \alpha>0, \phi_{f}$ decreases due to disorder that introduces bonds that are too strong to break before the matrix fails.

According to the prediction for the MS model in Equation 5.6 we can collapse all this data on one master curve for all values of $\lambda_{S}, \mu_{M} / \mu_{S}$ and $\delta \lambda$, if we consider the rescaled distance to the BDT: $\Delta \tilde{\alpha}$. Indeed, we obtain a good collapse for $\phi_{f}$, as shown in Figure 5.6(b). However, there is a significant increase in $\phi_{f}$ in the brittle regime relative to the prediction of the MS model (dashed line) (Figure 5.A.5). 
(a)

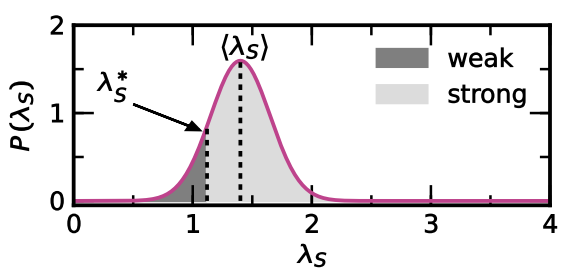

(d)

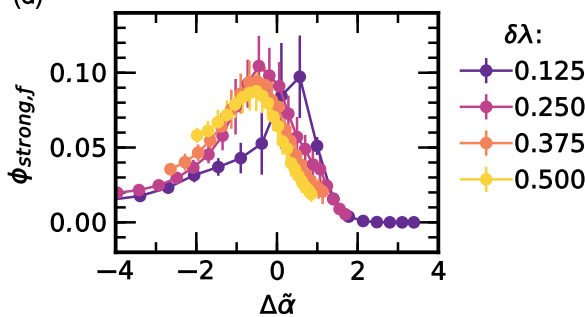

(b)
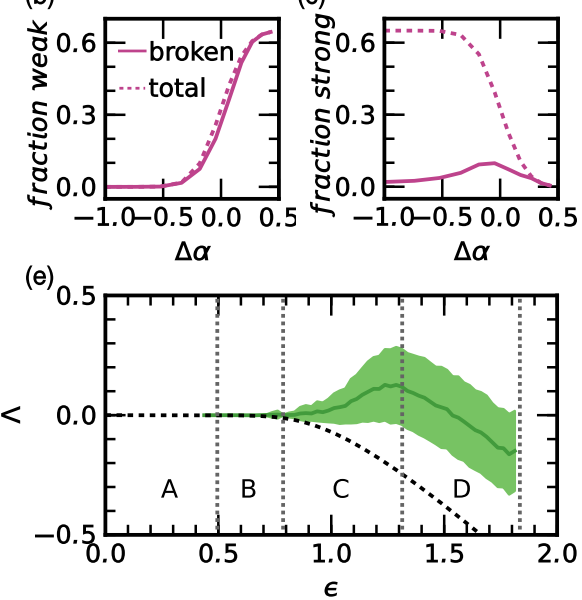

Figure 5.7. Development of stress heterogeneity and stress concentration during deformation. In all subpanels $\left\langle\lambda_{S}\right\rangle=1.4$. (a) Distribution of $\lambda_{S}$ for a system with $\left\langle\lambda_{S}\right\rangle=1.4$ and $\Delta \tilde{\alpha}=-0.7$. At $\lambda_{S}^{*}$ the force required to break a sacrificial bond is equal to the force required to break a matrix bond. Thus, if $\lambda_{S}<\lambda_{S}^{*}$ a sacrificial bond is weaker than the matrix bonds (dark grey) and if $\lambda_{S}>\lambda_{S}^{*}$ a sacrificial bond is stronger (light grey). (b) Comparison of total fraction of weak bonds that align with the deformation field and the fraction of weak bonds $\phi_{\text {weak, } f}$ that failed during deformation versus $\Delta \alpha$. (c) Similar comparison for the total amount of strong bonds

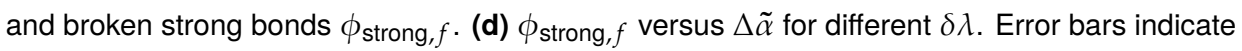
standard deviation. (e) Development of $\Lambda$, defined as the difference between the rupture threshold $\lambda_{S \text {,fail }}$ of the failing sacrificial bonds and $d \ell_{\text {aff }}$, the extension of the bonds if the strain field were homogeneous throughout the system, as a function of strain for a network with $\Delta \tilde{\alpha}=-0.7$, $\left\langle\lambda_{S}\right\rangle=1.4$ and $\delta \lambda=0.250$. The four intervals are described in the text. The average (line) and standard deviation (shaded area) are calculated from a histogram of $\lambda_{S \text {,fail }}-d \ell_{\text {aff }}$ over 50 simulations accumulated over bins of $2.5 \%$ width. The dashed black line indicates the prediction for $\Lambda$ according to the MS model.

This could be an indication that the load sharing via the network enhances the rupture of sacrificial bonds; however, we can not exclude that the discrepancy is due to a finite size effect.

\section{Stress concentration in the spring network model}

In the MS model it is assumed that stress is distributed homogeneously throughout the entire system, even after rupture of sacrificial bonds. Hence, stress can never concentrate within the MS model. In the RSN model stress concentration is possible 
and, therefore, it can be used to study the nucleation and propagation of cracks in double networks. It is fascinating that this fundamental difference between the MS model and the RSN model does not result in big differences in global failure or the fraction of broken sacrificial bonds.

Interestingly, a second look at the type of sacrificial bonds that break does reveal a striking difference between the MS model and the RSN model caused by the concentration of stress. By construction, in the MS model sacrificial bonds break in the order of their strength, from weak to strong, until a certain threshold $\lambda_{S}^{*}$ (Equation 5.3) is reached and global failure occurs. At this threshold, $\lambda_{S}^{*}$, the force required to break a (matrix-reinforced) sacrificial bond is equal to the force required to break a matrix bond. How $\lambda_{S}^{*}$ influences the rupture of bonds is further illustrated in Figure 5.7(a) for a system below the BDT ( $\Delta \tilde{\alpha}=-0.7, \mu_{M}=0.4$ and $\left.\left\langle\lambda_{S}\right\rangle=1.4\right)$. For this system $\lambda_{S}^{*}=1.14$, so in the MS model only weak bonds with $\lambda_{S}<1.14$ would fail. However, in the RSN model we see that sacrificial bonds stronger than $\lambda_{S}^{*}$ also break (Figure 5.7(b) and (c)). In particular, in Figure 5.7(d) we

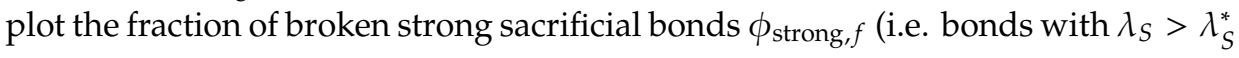
that break) as a function of the distance from the BDT $\Delta \tilde{\alpha}$ for various amounts of disorder $\delta \lambda$. At low $\delta \lambda$ and far below the BDT, rupture of strong sacrificial bonds is required in order to achieve global failure, since there are just not enough weak sacrificial bonds (Figure 5.7(b)). However, upon approaching the transition at $\Delta \tilde{\alpha}=0, \phi_{\text {strong, } f}$ grows, even though a decrease could be expected based on the total concentration of strong sacrificial bonds (Figure 5.7(c)). Interestingly, we observe a maximum in $\phi_{\text {strong, } f}$ before fully entering the ductile regime $\Delta \tilde{\alpha}>0$ where mostly weak sacrificial bonds control the fracture process. Strong bonds can only break if the force they carry exceeds the maximum force that is expected based on the macroscopic force balance. Thus, we conclude that the network structure plays a crucial role in (re)distributing the load during deformation, giving rise to stress concentration. The development of stress heterogeneity is tightly bound to the disorder that is present in the initial structure. We expect that by including more (structural) disorder in the initial network (e.g. inhomogeneities in the connectivity of the nodes, a disordered spatial distribution of the nodes, or a distribution in the stiffness of the bonds) the stress heterogeneity would become even larger ${ }^{33}$. As a consequence, we would expect significant broadening of the QB and D1 phase with respect to the prediction from the MS model.

Because in the RSN the initial structure is homogeneous, stress concentration does not occur from the onset of deformation, but develops during deformation as bonds are ruptured. We can quantify this development via the difference between the rupture threshold of a broken sacrificial bond $\lambda_{S \text {, fail, a measure for }}$ the actual local extension, and the extension estimated from the global strain 
assuming all deformations are affine, $d \ell_{\text {aff }}$. We, therefore, define $\Lambda=\lambda_{S \text {,fail }}-d \ell_{\text {aff }}$. To compare with the MS model, it must be noted that in this model the stress is homogeneous, but the strain field is not. In fact, the microcracks take up additional strain to compensate for the absence of the sacrificial network. This means that the deformation of the remaining sacrificial bonds is less than the affine strain, so that $\Lambda$ is always smaller that 1 in the MS model (see dashed line in Figure 5.7(e)). If at any time $\Lambda>0$, stress concentration must, therefore, be present.

This analysis reveals different modes of stress concentration during the fracture of a RSN system as shown in Figure 5.7(e). At first nothing breaks, this regime coincides with the reversibly elastic regime found at the macroscopic level (regime A). Then, sacrificial bonds start to break exactly as predicted by the affine deformation. Here both the average stress concentration, $\langle\Lambda\rangle$, and its variance are zero (regime B). Upon further increasing the deformation, the variance of $\Lambda$ starts to grow indicating stress heterogeneity in the system, quickly followed by an increase of $\langle\Lambda\rangle$ indicating stress concentration (regime $\mathrm{C}$ ). As a result strong sacrificial bonds also start to break and rupture of weak sacrificial bonds is postponed or even prevented (Figure 5.A.6). Finally, we arrive at a peak in $\langle\Lambda\rangle$, after which the $\langle\Lambda\rangle$ decreases until global failure is reached (regime $\mathrm{D}$ ). We attribute this decrease to structural relaxation of the sacrificial network via the formation of microcracks, leading to loss of rigidity and eventually loss of percolation in the sacrificial network. It must be noted that the variance in $\langle\Lambda\rangle$ remains constant, indicating that heterogeneity in stress is present until the final failure event.

\section{Crack development and stress delocalization}

The development of stress heterogeneity and stress concentration during deformation, is the result of microscopic damage evolution in the sacrificial network: the nucleation and propagation of cracks. We also suggested that the development of stress concentration is tightly linked to the disorder in the initial, undeformed, system. How crack nucleation and propagation are influenced by both initial disorder and stress heterogeneity during deformation is not trivial. We have already seen that in the RSN model not all sacrificial bonds that break are part of the largest crack (Figure 5.4). Thus, it seems that stress can be delocalized away from the crack tip of the largest crack throughout the system via diffuse damage. In this section, we quantify the evolution of (micro)cracks in the sacrificial network, focusing on diffuse damage and its coupling with stress delocalization.

In Figure 5.8(a), we plot the diffuse damage, quantified by the fraction of broken sacrificial bonds (with respect to the total number of initial sacrificial bonds) that are not part of the largest crack $\phi_{\text {diff }}$ as a function of strain $\varepsilon$ for three representative 
(a)

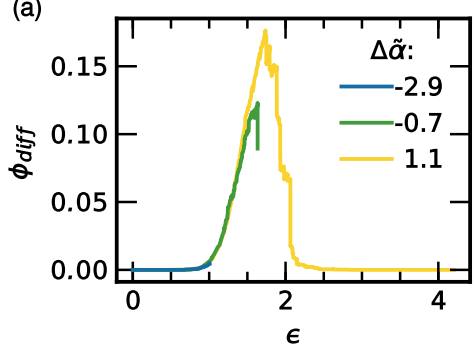

(b)

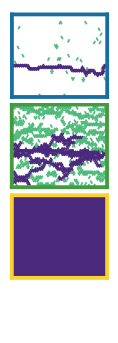

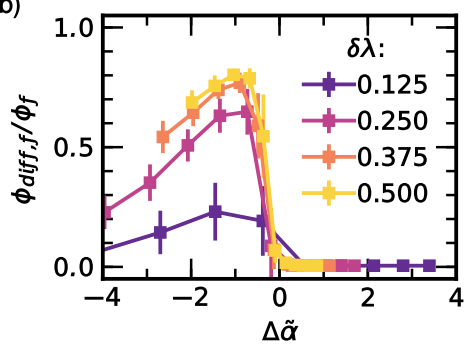

Figure 5.8. Evolution of diffuse damage. (a) Fraction of broken sacrificial bonds not belonging to the largest crack ( $\phi_{\text {diff }}$ ) as a function of strain $\varepsilon$ for different combinations of parameters below $(\Delta \tilde{\alpha}<0)$ and above $(\Delta \tilde{\alpha}>0)$ the BDT. Corresponding fracture patterns in the sacrificial network are also shown, with purple bonds belonging to the largest crack and green bonds to diffuse damage. (b) Normalized diffuse damage at global failure $\phi_{\text {diff, } f}$ as a function of $\Delta \tilde{\alpha}$ for different disorder $\delta \lambda$. Points are averages and error bars indicate standard deviation calculated over 50 configurations. For all curves $\left\langle\lambda_{S}\right\rangle=1.4$.

systems below, around and above the BDT (see Section 5.2.4 for details). In the brittle case $(\Delta \tilde{\alpha}=-2.9), \phi_{\text {diff }}$ barely increases and fracture occurs at smaller strain. Closer to the $\operatorname{BDT}(\Delta \tilde{\alpha}=-0.7)$, the diffuse damage significantly increases, reaches a maximum, and just before global failure slightly decreases. This decrease in the curve indicates that microcracks merge into the largest crack that eventually breaks the system (Figure 5.8(a)). Beyond the BDT $(\Delta \tilde{\alpha}=1.1)$, the diffuse damage reaches a higher maximum of more than $15 \%$ of the total amount of sacrificial bonds before a significant decrease that extends for a large strain interval. Finally, $\phi_{\text {diff }}$ reaches zero, indicating that all microcracks are now merged in a single large crack or damage zone, well before global failure and all subsequent breaking sacrificial bonds join this large crack.

With respect to the global stress-strain response, diffuse damage is expected to be most influential before the BDT. In the ductile regime, broken bonds will eventually join to form one large damage zone. However, in the brittle regime, many bonds can break in a diffuse fashion without being part of the largest crack, potentially diminishing stress concentration. We, therefore, focus on the final ratio of the broken sacrificial bonds belonging to the diffuse damage $\phi_{\operatorname{diff}, f}$ as an indicator of stress delocalization and plot it versus $\Delta \tilde{\alpha}$ for various amounts of disorder in Figure 5.8(b). We observe a non-monotonic behaviour with a peak slightly before the BDT. At the BDT $\phi_{\text {diff, } f}$ drops drastically as all bonds eventually join the largest crack. We conclude that just before the BDT, the diffuse damage is maximum and, therefore, hypothesize that the crack nucleation is delayed the 


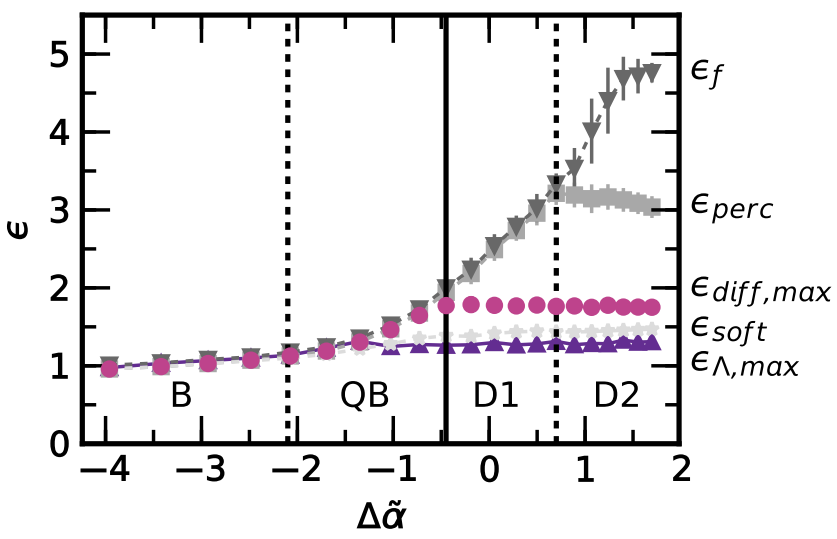

Figure 5.9. Characteristic strains corresponding to microscopic and macroscopic events as a function of $\Delta \tilde{\alpha}\left(\left\langle\lambda_{S}\right\rangle=1.4\right.$ and $\left.\delta \lambda=0.250\right)$. $\varepsilon_{\Lambda \text {,max }}$ (purple triangles) is the strain at maximum

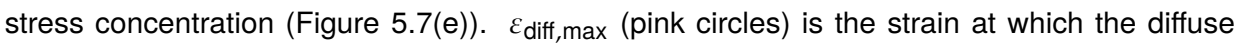
damage is maximal (Figure 5.8(a)). $\varepsilon_{\text {perc }}$ (grey squares) is the strain at which percolation in the sacrificial network is lost. $\varepsilon_{\text {soft }}$ (light grey downward triangles) indicates the onset of softening in the stress-strain curve (Figure 5.4(a) and Section 5.2.3). $\varepsilon_{f}$ (dark grey stars) corresponds to final fracture. The vertical lines indicate the transitions between the fracture regimes in the RSN estimated from the phase diagram in Figure 5.5(b).

most in this region. A consequence of this delayed nucleation is the widening and branching of the largest crack as shown in Figure 5.8 (a), snapshot in the middle. The widening of the damage zone in the sacrificial network around a defect is often used as an explanation for the enhanced fracture toughness in double network materials ${ }^{2,18}$. Although our simulations do not measure the fracture toughness directly, our results suggest that widening of the damage zone and, therefore, fracture toughness is maximal just before the BDT.

\subsubsection{Relating microscopic events to the macroscopic fracture regimes}

The network and the heterogeneous stress distribution emerging upon deformation, clearly have a huge impact on the microscopic fracture mechanism. We have seen that which microscopic fracture mechanisms occur is controlled by the distance from the BDT. In addition, we have observed that these fracture mechanisms can be connected to fracture events that occur as a function of the applied strain. Here we will unify our microscopic insights with the macroscopic fracture regimes described earlier based on the strain values at which these events occur. These results are summarized in Figure 5.9. 
At the macroscopic level we identify two characteristic strains: the softening strain $\varepsilon_{\text {soft, }}$ where the stiffness $(d \sigma / d \varepsilon)$ drops below the initial stiffness (Figure 5.4(a)), and the failure strain $\varepsilon_{f}$ where the system breaks in two pieces. At the microscopic level we identify strain points of three distinct events: the strain $\varepsilon_{\Lambda, \max }$ corresponding to the maximum in stress concentration (peak in Figure 5.7(e)), the strain where the diffuse damage is maximal $\varepsilon_{\text {diff,max }}$, corresponding to the peak in Figure 5.8(a), and finally the strain where the (geometrical) percolation of the network of sacrificial bonds is lost $\varepsilon_{\text {perc }}$ (i.e. the top and bottom of the sacrificial network are no longer connected).

$B$ regime - In the brittle regime, far below the $\mathrm{BDT}$, the system deforms homogeneously and every microcrack immediately develops into a macroscopic crack, leading to global failure. Therefore, all fracture events, both macroscopically and microscopically, occur at the failure strain $\varepsilon_{f}$.

$\mathrm{QB}$ regime - Approaching the $\mathrm{BDT}$ from below, we arrive at the $\mathrm{QB}$ regime, where global failure is preceded by softening in the stress-strain curve. The onset of this softening behaviour $\varepsilon_{\text {soft }}$ becomes distinguishable from the failure strain $\varepsilon_{f}$ around the transition from B-to-QB. Interestingly, the softening strain does not depend strongly on the distance from the BDT, $\Delta \tilde{\alpha}$ (Figure 5.9). If we direct our attention to the microscopic level, we see that the onset of softening coincides with the peak in stress concentration $\Lambda$ (Figure 5.7(e)). Also for $\varepsilon_{\Lambda, \max }$ we only see a weak dependence on the distance from the BDT (Figure 5.9). Here we would like to stress that even though the softening strain is the first clear macroscopic sign of fracture, it is not the strain at which the first bonds break. Actually, a significant amount of (weak) bonds break homogeneously throughout the system before the softening strain. However, the creation of these microcracks does not have a big effect on the stress-strain response because the bonds surrounding these microcracks absorb the released stress, leading to the increase in stress concentration in Figure 5.7(e). At the softening strain, such a large amount of microcracks has been formed that the system can release (a part of) the stress caused by an applied strain via structural relaxation. i.e. the rearrangement of nodes and springs, causing the softening in the stress-strain curve in Figure 5.4(a) and the drop in $\Lambda$ in Figure 5.7(e). Also after $\varepsilon_{\text {soft }}$, we mostly observe the creation of new microcracks or a minor expansion of existing microcracks as indicated by the strong rise of diffuse damage (Figure 5.8). However, in the QB regime, just before global failure, most of these microcracks merge into one large macroscopic crack, leading to global failure as is visible in the middle snapshot in Figure 5.8.

$D 1$ regime - After the $\mathrm{BDT}$, the merging of microcracks starts to occur well before system failure, as can be identified from a peak at $\varepsilon_{\text {diff,max }}$ in the diffuse damage as a function of strain (Figure 5.8(a)). In the ductile regime, the merging of cracks 
occurs at the same strain, as indicated by the fact that $\varepsilon_{\text {diff,max }}$ is independent of the distance from the BDT. Even though microscopic cracks in the sacrificial network start to merge, this does not mean that the sacrificial network breaks completely, i.e. loses (geometrical) percolation. In fact, in the D1 regime for a system with sufficient disorder in $\lambda_{S}$ loss of percolation in the sacrificial network only occurs around the failure strain (Figure 5.9). Thus, in a system with a network structure such as the RSN, the criterion for entering the plateau region in the stress-strain curve (Figure 5.4) does not have to be loss of percolation in the sacrificial network, but rather the opportunity for structural relaxation within the sacrificial network. This observation provides an alternative mechanism with respect to what was proposed in literature, where loss of percolation was thought to be necessary to enter the ductile or necking regime ${ }^{9,34}$.

D2 regime - In the D2 regime, the stress-strain curve enters a matrix dominated regime after the plateau in stress (Figure 5.4(a)). Microscopically this transition is marked by the loss of percolation in the sacrificial network. From the loss of percolation onward, the stress in the material is carried by the matrix as is also apparent from the constant slope in the stress-strain curve (Figure 5.4(a)). However, we do also observe that $\varepsilon_{f}$ continues to grow with increasing $\Delta \tilde{\alpha}$, until a plateau is reached in the failure strain as we have observed earlier in Figure 5.3. The initial increase of $\varepsilon_{f}$ in the D2 regime, shows that although percolation is lost in the sacrificial network and the matrix carries most of the stress, the sacrificial network can still contribute to the mechanical properties. Finally, we emphasize that during all these processes the matrix network remains largely intact and only very close to global failure the matrix bonds break (Figure 5.A.2).

\subsubsection{Connecting the model to experiments}

In our model, we explored how stiffness $\left(\mu_{M}\right.$ and $\left.\mu_{S}\right)$ and extensibility (strain at break of a single spring $\lambda_{M}$ and $\lambda_{S}$ ) of the individual networks influence the fracture behaviour of double networks. Typically, in an experimental system, such as a hydrogel or an elastomer, those parameters cannot be tuned independently. For example, the stiffness is set by the density of polymer chains ${ }^{35}$ and the extensibility is set by the length of these chains ${ }^{36}$. As a first approximation, the volume fraction of monomers determines the density of polymer chains, and thus the stiffness $(\mu)$, whereas the ratio between the volume fraction of monomer and crosslinker determines the chain length, and thus the extensibility $(\lambda)$. This is only an approximation, because at high monomer volume fractions both intra- and inter-network entanglements will occur. These entanglements can act as effective 
crosslinks, increasing the effective chain density and reducing the effective chain length ${ }^{37,38}$.

Nevertheless, within this approximate mapping, we find that experimental results for hydrogel and elastomer double networks are qualitatively consistent with the predictions of our model. For example, hydrogels show a transition from a brittle to a ductile fracture response if the volume fraction of the matrix monomer

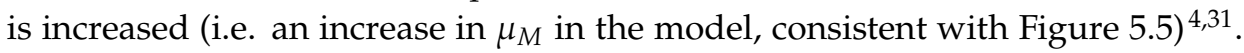
Moreover, experiments on hydrogels and elastomers reveal that an effective way to make a tough double network is to swell the sacrificial network $3,13,16,31$, which pre-stretches the polymer chains in the sacrificial network. The pre-stretching reduces the extensibility of the sacrificial network, which can be captured in our model by decreasing $\lambda_{S}$. In agreement with experiments, we observe in Figure 5.5 that by reducing $\lambda_{S}$ we can enter the ductile fracture regime. If the matrix monomers are used to swell the network, both $\lambda_{S}$ and $\mu_{M} / \mu_{S}$ are affected, revealing that the experimental swelling protocol ultimately determines which regions of the phase diagrams in Figure 5.5 can be explored. Furthermore, our model shows that disorder tunes the macroscopic fracture response away from the $\mathrm{BDT}$, such as the B-to-QB transition and the D1-to-D2 transition. In experiments, the introduction of voids can be one method to introduce disorder in a controlled way. For example, in Ref. [39] a shift in the fracture response is observed from D2 to D1 with an increase in void size. In the context of our model, this corresponds to an increase in disorder and can be explained as a decrease in $\Delta \tilde{\alpha}$ (Equation 5.7).

Finally, recent advances in mechanochemistry made it possible to explore the fracture response at the microscopic level as well, revealing significant diffuse damage, even before the yielding point ${ }^{12,13}$ (Figure 5.7). In addition, scattering experiments show evidence of the delocalization of stress by probing the length scale of the non-affine response ${ }^{40}$ and the expansion of microscopic defects ${ }^{41}$. In particular Ref. [41] suggests that a higher fraction of matrix monomer leads to the creation of new microcracks instead of the expansion of existing defects (Figure 5.8). These observations fit within our theoretical framework where the distance from the BDT and disorder provide control over the microscopic fracture mechanism.

The current simulation framework can be extended to increase the accuracy of its predictions for polymeric systems such as hydrogels and elastomers. In particular, we expect that the implementation of non-linear springs will better represent the non-linear stress-strain response of finitely extensible polymers and the redistribution of stress in a polymer network. With such an extended model we could explore if non-linear elasticity enhances stress delocalization with 
respect to linear elasticity and if this effect influences nucleation and propagation of microcracks.

\subsection{Concluding remarks}

In this chapter we studied both the macroscopic and microscopic fracture behaviour of double network materials using a spring network model (RSN) with emergent load sharing. By comparing the results of the RSN with a model based on equal load sharing, we reveal that: (i) The location of the BDT, defined on the basis of macroscopic stress-strain behaviour, is captured by a simple force balance. (ii) Disorder introduces intermediate fracture regimes but it can be incorporated in the parameter $\Delta \tilde{\alpha}$ to correctly describe the distance from the BDT, allowing for rescaling of the number of total broken sacrificial bonds. (iii) At the microscopic level stress concentration and delocalization reveal a markedly different picture compared to global load sharing.

The overall picture that emerges from the RSN is that the force balance, a central feature of double networks, has significant control over both the macroscopic and microscopic fracture behaviour, irrespective of how stress is (re)distributed. By contrast, the nucleation and propagation of (micro)cracks is also highly dependent on the mode of stress (re)distribution. In particular, we have identified how stress concentration, diffuse damage, and loss of percolation are related to the transitions from B-to-QB, QB-to-D1, and D1-to-D2, respectively.

We highlight that, because many microcracks can form before global failure due to the stabilization by the matrix, the load sharing in double networks becomes highly non-linear as a result of the interaction between these microcracks. Therefore, double networks provide a unique opportunity to exploit these non-linearities in microcrack interaction. For example, this knowledge about the microscopic fracture process could aid the development of robust self-healing double networks, as the healing of diffuse damage is easier than the healing of macroscopic cracks ${ }^{11,42,43}$.

By extending the RSN model, additional features of double network gel fracture could be studied, such as the influence of pre-stress or structural disorder in the sacrificial network. Furthermore, the introduction of disorder in the matrix would allow to also study the enhancement of strength and toughness, including the role of microscopic fracture. In conclusion, this chapter demonstrates that a random spring network model provides the opportunity to systematically study the microscopic fracture process within double networks. 


\section{A Appendix}

(a)

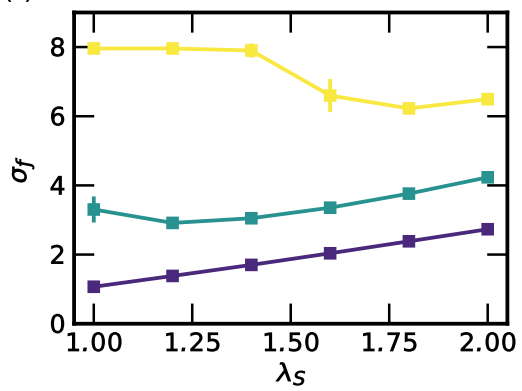

(b)

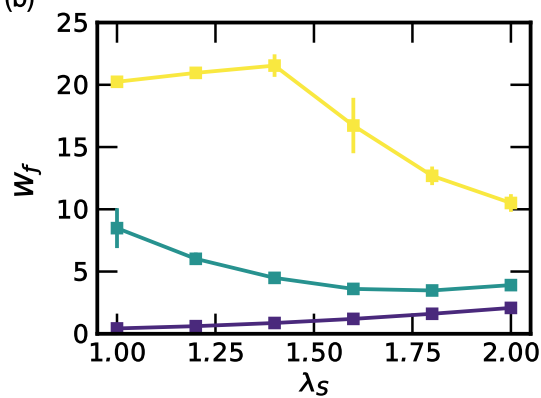

Figure 5.A.1. Material properties versus the average rupture threshold $\left\langle\lambda_{S}\right\rangle . \quad(\delta \lambda=0.250$, $L=50$ ). (a) Failure stress, $\sigma_{f}$. (b) Work of extension $W_{f}$, the area under the stress-strain curve until global failure. Results are shown for stiffness ratios $\mu_{M} / \mu_{S}=0.10$ (purple), 0.50 (green) and 1.00 (yellow).
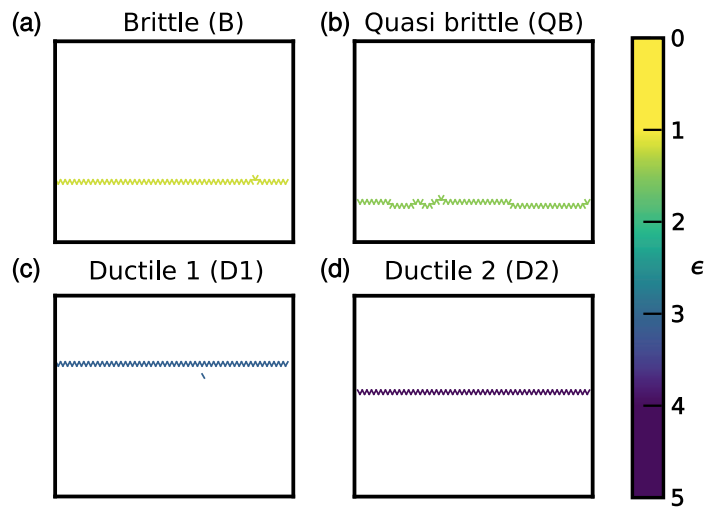

Figure 5.A.2. Spatial damage in the matrix network for the RSN model. (a)-(d) Failure patterns in the matrix network for the four distinct fracture behaviours. Every line represents a broken matrix bond, colour coded according to the strain at which the bond fails (see colour bar above). 
(a)

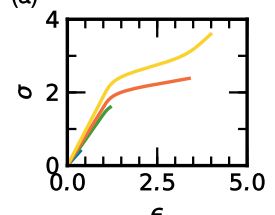

(e)

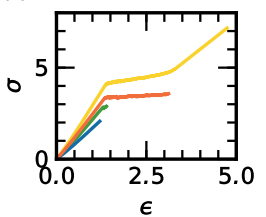

(b)

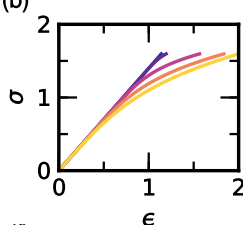

(f)

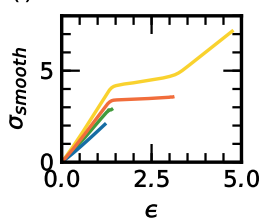

(c)

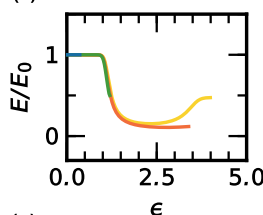

(g)

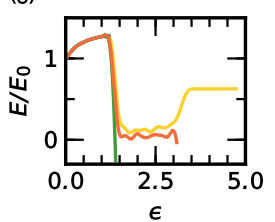

(d)

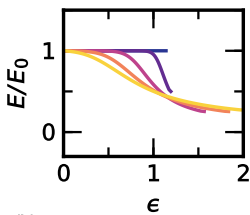

(h)

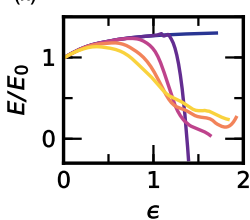

Figure 5.A.3. Stress-strain classification based on stiffness for both the MS model ((a)-(d)) and the RSN model ((e)-(h)). For the MS model we show in panel (a) the stress-strain curves for stiffness ratios $\mu_{M} / \mu_{S} 0.1$ (blue), 0.4 (green)), 0.6 (orange) or 0.8 (yellow) $\left(\left\langle\lambda_{S}\right\rangle=1.4, \delta \lambda=0.125\right.$ ). (b) the stress-strain curves for different amounts of bond strength disorder $\delta \lambda$ from 0.000 (blue) to 0.500 (yellow) $\left(\left\langle\lambda_{S}\right\rangle=1.4, \mu_{M} / \mu_{S}=0.4\right)$. panels (c) and (d) show the stiffness corresponding to panels (a) and (b) normalized by the initial stiffness $E_{0}$. For the RSN model we show in panel (e) stress-strain curves for a series of stiffness ratios (see description of panel (a) for details). (f) the same stress-strain curves smoothed according to the description in section 5.2.3. (g) the normalized stiffness corresponding to the stress-strain curves in panel (f). (h) the normalized stiffness of for different amounts of disorder (see description of panel (b) for details).
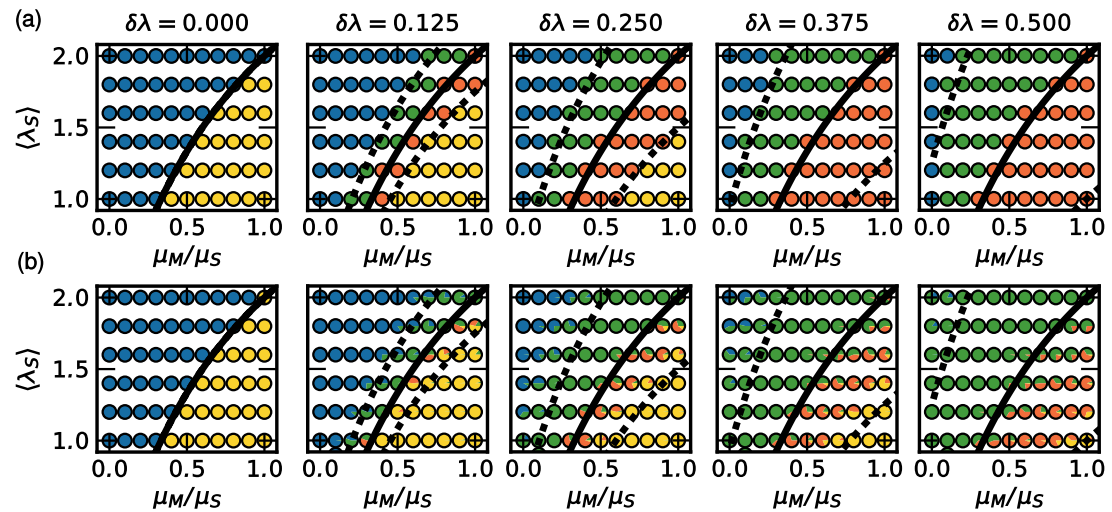

Figure 5.A.4. Stress-strain response of both the MS model (panel (a)) and the RSN model (panel (b)) in the $\left(\mu_{M} / \mu_{S}, \lambda_{S}\right)$-plane for different amounts of disorder $\delta \lambda$. See 5.2.3 for more information. The colours correspond to the fracture regimes: brittle (blue), quasi-brittle (green), ductile 1 (orange) and ductile 2 (yellow). The black line indicates the BDT transition as predicted by the MS model, the dotted lines are placed at $\Delta \tilde{\alpha}=-2.0$ and $\Delta \tilde{\alpha}=2.5$ corresponding to the estimated B-to-QB and D1-to-D2 transitions in the MS model respectively. 
(a)

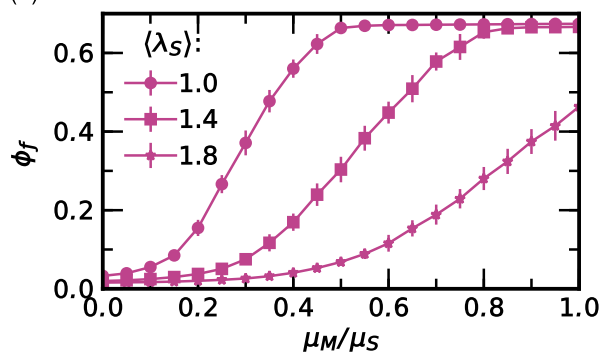

(b)

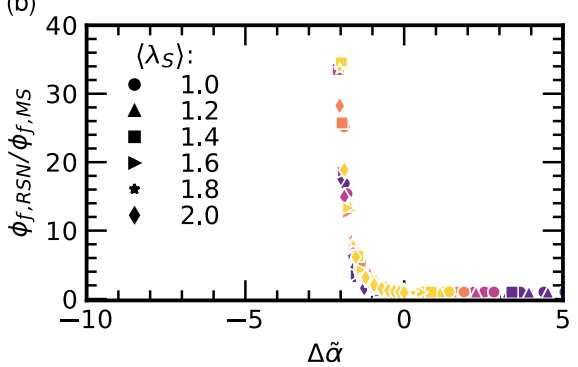

Figure 5.A.5. (a) Fraction of broken sacrificial bonds in the RSN model after global failure $\phi_{f}$ as a function of $\mu_{M} / \mu_{S}$ for several $\left\langle\lambda_{S}\right\rangle$ (see legend). In this panel $\delta \lambda=0.250$ and $L=50$. (b) The relative increase in $\phi_{f, \mathrm{RSN}}$ with respect to $\phi_{f, \mathrm{MS}}$ plotted versus $\Delta \tilde{\alpha}$ for a range of $\lambda_{S}$ (marker shape) and a range of $\delta \lambda$ (marker colour).)

(a)

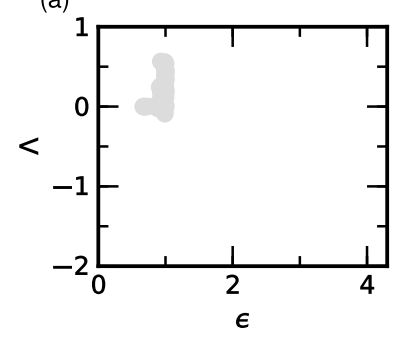

(b)

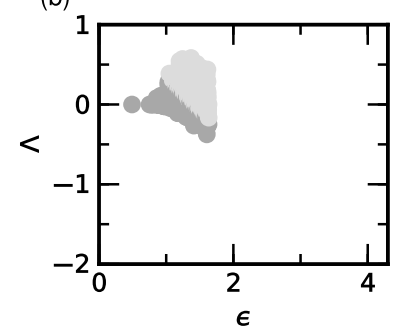

(c)

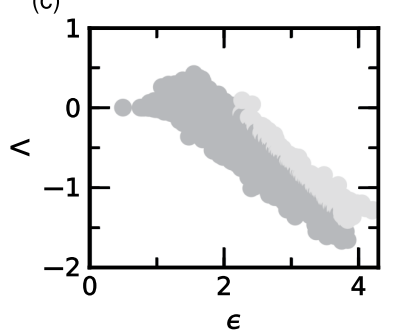

Figure 5.A.6. Rupture of weak and strong bonds. For a single simulation $\Lambda=\lambda_{S \text {,fail }}-d \ell_{\text {aff }}$ is plotted versus $\varepsilon$. Every dot represents a broken sacrificial bond and the colour indicates if the bond is strong (light grey) or weak (dark grey). In all panels $\left\langle\lambda_{S}\right\rangle=1.4$ and $\delta \lambda=0.250$. (a) $\mu_{M} / \mu_{S}=0.1$. (b) $\mu_{M} / \mu_{S}=0.4$. (c) $\mu_{M} / \mu_{S}=0.8$. 


\section{List of symbols}

\begin{tabular}{|c|c|}
\hline Symbol & Description \\
\hline E & Stiffness, defined as $E=d \sigma / d \epsilon$ \\
\hline$E_{0}$ & Linear stiffness \\
\hline$F_{\mathrm{rms}}$ & Tolerance, maximum root mean square force allowed in the system \\
\hline$\Delta \ell$ & $\begin{array}{l}\text { Extension (or compression) of a spring with respect to its rest } \\
\text { length } \ell_{0}\end{array}$ \\
\hline$d \ell_{\text {aff }}$ & Bond extension expected based on affine deformation \\
\hline$L$ & Lattice size, with $L \times L$ the number of nodes \\
\hline$N$ & System size of the multi-spring model \\
\hline$W_{f}$ & Work of extension \\
\hline$\Delta \alpha$ & $\begin{array}{l}\text { Distance from the brittle-to-ductile transition based on average } \\
\text { rupture forces }\end{array}$ \\
\hline$\Delta \tilde{\alpha}$ & $\begin{array}{l}\text { Normalized distance from the brittle-to-ductile transition includ- } \\
\text { ing the effect of disorder in the sacrificial rupture threshold }\end{array}$ \\
\hline$\epsilon$ & Uniaxial strain \\
\hline$\epsilon_{\text {diff,max }}$ & Strain at which diffuse damage in the sacrificial network is maximal \\
\hline$\epsilon_{f}$ & Failure strain \\
\hline$\epsilon_{\text {perc }}$ & $\begin{array}{l}\text { Strain at which geometric percolation is lost in the sacrificial } \\
\text { network }\end{array}$ \\
\hline$\epsilon_{\text {soft }}$ & Softening strain \\
\hline$\epsilon_{\Lambda, \max }$ & Strain at which the stress concentration indicator $\Lambda$ is maximal \\
\hline$\lambda_{M}$ & Rupture threshold of elements in the matrix network \\
\hline$\lambda_{S}$ & Rupture threshold of elements in the sacrificial network \\
\hline$\left\langle\lambda_{S}\right\rangle$ & Mean rupture threshold of elements in the sacrificial network \\
\hline$\lambda_{S}^{*}$ & $\begin{array}{l}\text { Rupture threshold at which the rupture force of a (matrix- } \\
\text { reinforced) sacrificial bond is equal to the rupture force of a } \\
\text { matrix bond }\end{array}$ \\
\hline$\delta \lambda$ & $\begin{array}{l}\text { Disorder, defined as the standard deviation in the rupture } \\
\text { threshold distribution of the sacrificial bonds }\end{array}$ \\
\hline$\Lambda$ & $\begin{array}{l}\text { A measure for stress concentration around broken sacrificial bonds, } \\
\text { defined as the difference between the rupture threshold of a broken } \\
\text { sacrificial bond and the extension } d \ell_{\text {aff }} \text { expected based on affine } \\
\text { deformation }\end{array}$ \\
\hline$\mu_{M}$ & Spring constant of elements in the matrix network \\
\hline$\mu_{S}$ & Spring constant of elements in the sacrificial network \\
\hline
\end{tabular}




$\begin{array}{ll}\sigma & \text { Stress } \\ \sigma_{f} & \text { Failure stress, stress at global failure } \\ \phi & \begin{array}{l}\text { Fraction of broken sacrificial bonds with respect to the number of } \\ \text { sacricial bonds in the undeformed network }\end{array} \\ \phi_{f} & \begin{array}{l}\text { Fraction of broken sacrificial bonds at global system failure } \\ \phi_{\text {diff }}\end{array} \\ \phi_{\text {diff,f }} & \begin{array}{l}\text { Diffuse damage, defined as the fraction of broken sacrificial bonds } \\ \text { that is not part of the largest crack } \\ \phi_{\text {large }}\end{array} \\ \end{array}$

\section{References}

[1] C. Creton, 50th Anniversary Perspective: Networks and Gels: Soft but Dynamic and Tough. Macromolecules 50, 8297-8316 (2017).

[2] J. P. Gong, Why are double network hydrogels so tough? Soft Matter 6, 2583-2590 (2010).

[3] T. Nakajima, Y. Fukuda, T. Kurokawa, T. Sakai, U. I. Chung, J. P. Gong, Synthesis and fracture process analysis of double network hydrogels with a well-defined first network. ACS Macro Letters 2, 518-521 (2013).

[4] J. P. Gong, Y. Katsuyama, T. Kurokawa, Y. Osada, Double-network hydrogels with extremely high mechanical strength. Advanced Materials 15, 1155-1158 (2003).

[5] L. Weng, A. Gouldstone, Y. Wu, W. Chen, Mechanically strong double network photocrosslinked hydrogels from $\mathrm{N}, \mathrm{N}$-dimethylacrylamide and glycidyl methacrylated hyaluronan. Biomaterials 29, 2153-2163 (2008).

[6] J. Y. Sun, X. Zhao, W. R. Illeperuma, O. Chaudhuri, K. H. Oh, D. J. Mooney, J. J. Vlassak, Z. Suo, Highly stretchable and tough hydrogels. Nature 489, 133-136 (2012).

[7] T. Nakajima, H. Sato, Y. Zhao, S. Kawahara, T. Kurokawa, K. Sugahara, J. P. Gong, A universal molecular stent method to toughen any hydrogels based on double network concept. Advanced Functional Materials 22, 4426-4432 (2012).

[8] H. Xin, S. Z. Saricilar, H. R. Brown, P. G. Whitten, G. M. Spinks, Effect of first network topology on the toughness of double network hydrogels. Macromolecules 46, 6613-6620 (2013).

[9] T. Nakajima, T. Kurokawa, S. Ahmed, W. L. Wu, J. P. Gong, Characterization of internal fracture process of double network hydrogels under uniaxial elongation. Soft Matter $\mathbf{9}$, 1955-1966 (2013).

[10] Q. Chen, L. Zhu, L. Huang, H. Chen, K. Xu, Y. Tan, P. Wang, J. Zheng, Fracture of the physically cross-linked first network in hybrid double network hydrogels. Macromolecules 47, 2140-2148 (2014). 
[11] X. Yan, Q. Chen, L. Zhu, H. Chen, D. Wei, F. Chen, Z. Tang, J. Yang, J. Zheng, High strength and self-healable gelatin/polyacrylamide double network hydrogels. Journal of Materials Chemistry B 5, 7683-7691 (2017).

[12] E. Ducrot, Y. Chen, M. Bulters, R. P. Sijbesma, C. Creton, Toughening elastomers with sacrificial bonds and watching them break. Science 344, 186-189 (2014).

[13] P. Millereau, E. Ducrot, J. M. Clough, M. E. Wiseman, H. R. Brown, R. P. Sijbesma, C. Creton, Mechanics of elastomeric molecular composites. Proceedings of the National Academy of Sciences of the United States of America 115, 9110-9115 (2018).

[14] R. Takahashi, T. L. Sun, Y. Saruwatari, T. Kurokawa, D. R. King, J. P. Gong, Creating Stiff, Tough, and Functional Hydrogel Composites with Low-Melting-Point Alloys. Advanced Materials 30, e1706885 (2018).

[15] D. R. King, T. Okumura, R. Takahashi, T. Kurokawa, J. P. Gong, Macroscale Double Networks: Design Criteria for Optimizing Strength and Toughness. ACS Applied Materials and Interfaces 11, 35343-35353 (2019).

[16] T. Matsuda, T. Nakajima, Y. Fukuda, W. Hong, T. Sakai, T. Kurokawa, U. I. Chung, J. P. Gong, Yielding Criteria of Double Network Hydrogels. Macromolecules 49, 1865-1872 (2016).

[17] Y. Tanaka, A local damage model for anomalous high toughness of double-network gels. Epl 78, 56005 (2007).

[18] H. R. Brown, A model of the fracture of double network gels. Macromolecules 40, 3815-3818 (2007).

[19] X. Wang, W. Hong, Pseudo-elasticity of a double network gel. Soft Matter 7, 8576-8581 (2011).

[20] X. Zhao, A theory for large deformation and damage of interpenetrating polymer networks. Journal of the Mechanics and Physics of Solids 60, 319-332 (2012).

[21] M. Bacca, C. Creton, R. M. McMeeking, A Model for the Mullins Effect in Multinetwork Elastomers. Journal of Applied Mechanics 84, 121009 (2017).

[22] F. J. Vernerey, R. Brighenti, R. Long, T. Shen, Statistical Damage Mechanics of Polymer Networks. Macromolecules 51, 6609-6622 (2018).

[23] S. R. Lavoie, P. Millereau, C. Creton, R. Long, T. Tang, A continuum model for progressive damage in tough multinetwork elastomers. Journal of the Mechanics and Physics of Solids 125, 523-549 (2019).

[24] K. Okumura, Toughness of double elastic networks. Europhysics Letters 67, 470-476 (2004).

[25] Y. Kawauchi, Y. Tanaka, H. Furukawa, T. Kurokawa, T. Nakajima, Y. Osada, J. Ping Gong, Brittle, ductile, paste-like behaviors and distinct necking of double network gels with enhanced heterogeneity. Journal of Physics: Conference Series 184, 012016 (2009). 
[26] S. S. Jang, W. A. Goddard, M. Yashar, S. Kalani, Mechanical and transport properties of the poly(ethylene oxide)-poly (acrylic acid) double network hydrogel from molecular dynamic simulations. Journal of Physical Chemistry B 111, 1729-1737 (2007).

[27] W. Wang, Z. Zhang, T. Davris, J. Liu, Y. Gao, L. Zhang, A. V. Lyulin, Simulational insights into the mechanical response of prestretched double network filled elastomers. Soft Matter 13, 8597-8608 (2017).

[28] Y. Higuchi, K. Saito, T. Sakai, J. P. Gong, M. Kubo, Fracture Process of Double-Network Gels by Coarse-Grained Molecular Dynamics Simulation. Macromolecules 51, 3075-3087 (2018).

[29] K. Kovács, R. C. Hidalgo, I. Pagonabarraga, F. Kun, Brittle-to-ductile transition in a fiber bundle with strong heterogeneity. Physical Review E - Statistical, Nonlinear, and Soft Matter Physics 87, 1-8 (2013).

[30] S. Roy, S. Biswas, P. Ray, Modes of failure in disordered solids. Physical Review E 96, 063003 (2017).

[31] S. Ahmed, T. Nakajima, T. Kurokawa, M. Anamul Haque, J. P. Gong, Brittle-ductile transition of double network hydrogels: Mechanical balance of two networks as the key factor. Polymer 55, 914-923 (2014).

[32] E. Bitzek, P. Koskinen, F. Gähler, M. Moseler, P. Gumbsch, Structural relaxation made simple. Physical Review Letters 97, 170201 (2006).

[33] S. Dussi, J. Tauber, J. Van Der Gucht, Athermal Fracture of Elastic Networks: How Rigidity Challenges the Unavoidable Size-Induced Brittleness. Physical Review Letters 124, 18002 (2020).

[34] Y. H. Na, Y. Tanaka, Y. Kawauchi, H. Furukawa, T. Sumiyoshi, J. P. Gong, Y. Osada, Necking phenomenon of double-network gels. Macromolecules 39, 4641-4645 (2006).

[35] H. M. James, E. Guth, Theory of the elastic properties of rubber. The Journal of Chemical Physics 11, 455-481 (1943).

[36] G. Lake, A. Thomas, The strength of highly elastic materials. Proceedings of the Royal Society of London. Series A. Mathematical and Physical Sciences 300, 108-119 (1967).

[37] N. R. Langley, K. E. Polmanteer, Relation of elastic modulus to crosslink and entanglement concentrations in rubber networks. Journal of Polymer Science: Polymer Physics Edition 12, 1023-1034 (1974).

[38] H. Tsukeshiba, M. Huang, Y. H. Na, T. Kurokawa, R. Kuwabara, Y. Tanaka, H. Furukawa, Y. Osada, J. P. Gong, Effect of polymer entanglement on the toughening of double network hydrogels. Journal of Physical Chemistry B 109, 16304-16309 (2005).

[39] T. Nakajima, H. Furukawa, Y. Tanaka, T. Kurokawa, J. P. Gong, Effect of void structure on the toughness of double network hydrogels. Journal of Polymer Science, Part B: Polymer Physics 49, 1246-1254 (2011).

[40] E. Ducrot, H. Montes, C. Creton, Structure of Tough Multiple Network Elastomers by Small Angle Neutron Scattering. Macromolecules 48, 7945-7952 (2015). 
[41] K. Fukao, T. Nakajima, T. Nonoyama, T. Kurokawa, T. Kawai, J. P. Gong, Effect of Relative Strength of Two Networks on the Internal Fracture Process of Double Network Hydrogels As Revealed by in Situ Small-Angle X-ray Scattering. Macromolecules 53, 1154-1163 (2020).

[42] H. Jia, Z. Huang, Z. Fei, P. J. Dyson, Z. Zheng, X. Wang, Unconventional Tough DoubleNetwork Hydrogels with Rapid Mechanical Recovery, Self-Healing, and Self-Gluing Properties. ACS Applied Materials and Interfaces 8, 31339-31347 (2016).

[43] S. Liu, L. Li, Ultrastretchable and Self-Healing Double-Network Hydrogel for 3D Printing and Strain Sensor. ACS Applied Materials and Interfaces 9, 26429-26437 (2017). 



\section{Chapter 6}

\section{Sharing the load: stress redistribution governs fracture of polymer double networks}

The stress response of polymer double networks depends not only on the properties of the constituent networks, but also on the interactions arising between them. Here we demonstrate, via coarse-grained simulations, that both their global stress response and their microscopic fracture mechanics are governed by load sharing through these inter-network interactions. By comparing our results with affine predictions, where stress redistribution is by definition homogeneous, we show that stress redistribution is highly inhomogeneous. In particular, the affine prediction overestimates the fraction of broken chains by almost an order of magnitude. Furthermore, homogeneous stress distribution predicts a single fracture process, while in our simulations fracture of sacrificial chains takes place in two steps governed by load sharing within a network and between networks, respectively. Our results thus provide a detailed microscopic picture of how inhomogeneous stress redistribution after rupture of chains governs the fracture of polymer double networks.

\section{Justin Tauber, Lorenzo Rovigatti, Simone Dussi \& Jasper van der Gucht} "Sharing the load: stress redistribution governs fracture of polymer double networks" Macromolecules, accepted (2021) 


\subsection{Introduction}

By consecutively cross-linking two interpenetrating polymer networks, a composite material is created which is commonly referred to as a (polymer) double network $(\mathrm{DN})^{1,2}$. In many DNs, the two underlying networks do not share any internetwork crosslinkers and are only topologically constrained at the chain-level ${ }^{1,3}$. For this reason, DNs can be considered as a molecular composite ${ }^{4}$. DNs have attracted considerable interest due to the significant enhancement in their (linear) stiffness, strength, and fracture toughness compared to single networks (SNs) ${ }^{1,4-8}$. For example, through this procedure hydrogels can be constructed that have a mechanical response similar to that of an elastomer ${ }^{9}$.

Experiments reveal that stiff, strong, and tough DNs are created when the first network, or sacrificial network, is stiff and weak, while the second network, or matrix network, is soft and stretchable ${ }^{9}$. To make networks with these properties, one can vary the type and concentration of monomers and crosslinkers in both networks ${ }^{8}$. Additionally, these properties can be controlled by swelling the sacrificial network either by introducing a molecular stent ${ }^{3,10}$ or using the monomer of the second network ${ }^{2}$. Experiments on a range of systems, varying from elastomers $^{2,4}$ to macroscopic networks ${ }^{11}$, suggest that the mechanism through which the enhancement occurs is surprisingly general: sharing of load between the two networks via their topological constraints ${ }^{12}$.

The corresponding microscopic picture is that, due to the presence of the matrix chains, the expansion of a (microscopic) crack in a DN requires considerably more energy than in an $\mathrm{SN}^{2,5-7,13}$. As a consequence, fracture of sacrificial chains in DNs is less likely to lead to the formation of macroscopic cracks and thus global failure ${ }^{14}$. Instead, the load is transferred (partially) from the sacrificial network to the matrix network surrounding the broken sacrificial polymer chain ${ }^{4}$. Thus, in a DN more sacrificial chains can break prior to global failure compared to an SN. As the intact sacrificial chains in these DNs can still resist deformation, the work required for global failure of the material is increased significantly compared to the individual networks. This concept has been termed the sacrificial bond principle ${ }^{12}$ and is widely accepted as the main microscopic cause for the enhanced mechanical properties in the fracture regime. This microscopic picture for accumulation of damage is confirmed by experiments $2,13,15,16$ and simulations ${ }^{17}$. However, despite state-of-the art experimental techniques enabling the visualization of stress ${ }^{18}$, strain $^{19-21}$ and the accumulation of damage at the local level ${ }^{2,4,22}$, a thorough understanding of how the microscopic processes affect the global material response is still lacking. 
Several constitutive models have been put forward that provide a connection between the evolution of damage and the global stress response of a $\mathrm{DN}^{23-27}$. These models can be fitted to experimental data and are also used in the interpretation of the output of mechanophores, i.e. molecular probes that report on the rupture of bonds locally ${ }^{18}$. Some of these models ${ }^{25-27}$, referred to as statistical damage mechanics models, predict the global response from the evolution of chain-stretch with respect to an initial stretch distribution, assuming affine deformation and breaking of over-stretched chains. As a result, the global mechanical response of a DN predicted by these models is the sum of the responses of two (or more) independent and affinely deforming networks. Effectively, these models assume that (statistically or on average) the intra-network load distribution follows the global deformation and that inter-network load redistribution is negligible at the global level. However, in the case of DN mechanics these assumptions deserve some scrutiny, because at first glance they seem to be incompatible with the proposed DN toughening mechanism where inter-network load sharing plays an essential role. In this work we investigate to what extent the microscopic process of redistribution of load, both within and between networks, affects the global mechanical response.

To this end, we perform coarse-grained simulations of polymer networks, where load redistribution is intrinsically captured. To generate the DNs we replicate a swelling procedure in silico which is commonly used to make both hydrogel and elastomer DNs in experiment ${ }^{2,3,10}$. By deforming the networks we obtain information on both the global stress response and the local stress, the local strain and the accumulation of damage. We show that the in silico networks behave in accordance with their experimental counterparts. Subsequently, we compare these simulation results with the affine predictions for the global stress and local damage response. From this comparison we find that in our simulations the microscopic mechanism for damage accumulation differs significantly from the affine approximation, with the affine prediction overestimating the fraction of broken chains by almost an order of magnitude. Furthermore, we show that the accumulation of damage occurs in two steps, one controlled by load sharing within the sacrificial network and one by load sharing between the two networks. Finally, we show that in our simulations load sharing causes an enhanced global mechanical response, in contrast to the affine prediction. We conclude with a discussion of the implications of our findings for the microscopic picture of fracture in DNs and polymer networks in general. 
(a)

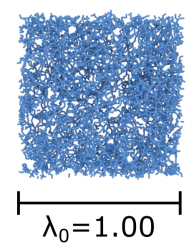

(c)

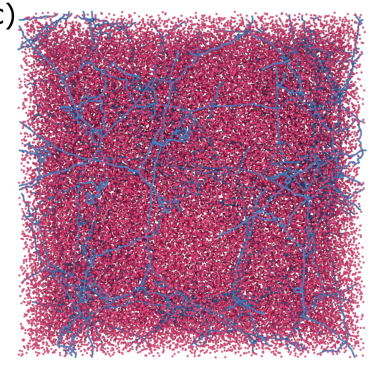

(b)

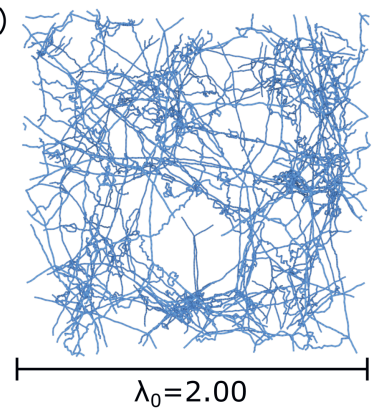

(d)

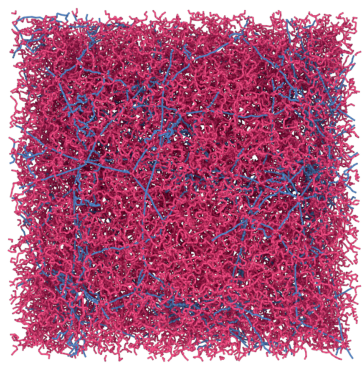

Figure 6.1. In silico double network generation. The sacrificial network is swollen from (a) $\lambda_{0}=1.00$ to (b) $\lambda=\lambda_{0}$. In this example $\lambda_{0}=2.00$. (c) Monomers for the matrix are added at random positions in the swollen sacrificial network such that the number density of the entire system equals $\rho$. (d) The matrix is formed within the sacrificial network with the same crosslinking procedure used for the sacrificial network, but with a lower crosslinker concentration.

\subsection{Model and Methods}

\subsubsection{In silico synthesis of double network}

The networks are formed by the self-assembly of binary mixtures of bifunctional and tetrafunctional patchy particles as done in Refs. [28-30]. In order to build the first network we simulate the binary mixture at a number density $\rho_{\text {init }}=0.17$. We stop the simulation when most (>99.9\%) of the bonds have formed, after which we remove the few clusters that are not attached to the largest one. No more than $3 \%$ of the particles are removed at this stage. We take the resulting system, locate all the chains, defined as clusters of bifunctional particles connecting the crosslinkers, and add five monomers to each in order to make the system more swellable (resulting in a number density $\rho_{\text {init,add }}=0.33$ ).

In order to swell the network in LAMMPS ${ }^{31}$, we convert the network of patchy particles to a network of harmonic bonds and equilibrate the network in the NVT ensemble for $10 \tau$, with $\tau$ the unit of time in our simulations. Subsequently, we 
convert the harmonic bonds to quartic bonds and equilibrate the network in the NPT ensemble for $100 \tau$ such that the network settles at an equilibrium box size $L_{\text {box }, 0}$. The resulting network is the sacrificial SN at $\lambda_{0}=1.00$ and number density $\rho=0.15$. This network is swollen isotropically (NVT ensemble) in steps of $\sim 0.1 \%$ strain such that the new box size is $L_{\text {box }}=L_{\text {box }, 0} \lambda_{0}$, providing the sacrificial SNs at higher swelling ratios.

To form the corresponding DNs, we add matrix monomers, and subsequently perform the same self-assembly procedure described above, with the difference that this time the bifunctional and tetrafunctional particles are embedded in the existing polymer network. After the assembly of the binary mixture completes we once again remove the few disconnected clusters but this time we do not add any additional monomers to the chains. The resulting DNs are NVT equilibrated in LAMMPS first using harmonic bonds (for $10 \tau$ ), then using quartic bonds (for $10 \tau$ ). Finally, the matrix SNs are obtained by removing the sacrificial network from the DNs.

For all LAMMPS simulations the time step for integration $d t=0.001 \tau$. For simulations performed in the NVT ensemble the temperature is controlled via a Nosé-Hoover thermostat and kept fixed at $T=1.0$ (in reduced units) with a damping time $t_{\text {damp }}=0.1 \tau$ (100 time steps). In addition, for simulations performed in the NPT ensemble the pressure is fixed at $P=1.0$ (in reduced units) and the corresponding $t_{\mathrm{damp}}=1.0 \tau$ (1000 time steps).

The interaction between the particles is described by the Weeks-ChandlerAndersen (WCA) potential, a truncated version of the Lennard-Jones potential,

$$
U(R)=4 \epsilon_{\mathrm{LJ}}\left[\left(\frac{\sigma_{\mathrm{LJ}}}{R}\right)^{12}-\left(\frac{\sigma_{\mathrm{LJ}}}{R}\right)^{6}\right] \quad R<R_{c},
$$

with $R$ the inter-particle distance, $\sigma_{\mathrm{LJ}}=1.0$ the particle diameter, $\epsilon_{\mathrm{LJ}}=1.0$ the depth of the potential well and $R_{c}=2^{1 / 6}$ the cut-off distance, unless the particles are connected by a bond, in that case the particle-particle interaction is described by a quartic potential $\left(K=2351, B_{1}=-0.7425, B_{2}=0.0, R_{c}=1.5, U_{0}=92.74467\right)$.

$$
U(R)=K\left(R-R_{c}\right)^{2}\left(R-R_{c}-B_{1}\right)\left(R-R_{c}-B_{2}\right)+U_{0}+4 \epsilon_{\mathrm{LJ}}\left[\left(\frac{\sigma_{\mathrm{LJ}}}{R}\right)^{12}-\left(\frac{\sigma_{\mathrm{LJ}}}{R}\right)^{6}\right]+\epsilon_{\mathrm{LJ}}
$$

These parameters have been used previously to study polymer rupture ${ }^{32}$. The quartic bonds break irreversibly if their extension exceeds $R_{c}=1.5$. However, the maximum force is already reached around an extension of 1.133 and the bonds are expected to break even earlier, around an extension of 1.08, due to thermal fluctuations (see Figure 6.A.6 for details). In some equilibration steps harmonic 
bonds are used instead of a quartic bond $\left(K=1000, R_{0}=0.96\right)$.

$$
U(R)=K\left(R-R_{0}\right)^{2}
$$

\subsubsection{Extension protocol}

The stress response is obtained by performing a continuous uniaxial extension at a strain-rate $\dot{\epsilon}=1 \times 10^{-4}$, while keeping the volume of the simulation box constant (lateral dimensions are reduced during extension). A similar procedure has been followed in literature 33,34 . Decreasing the strain-rate by a factor 10 does not significantly alter the mechanical response. The stress response $\sigma$ is determined from the virial stress excluding kinetic contributions, which are nevertheless negligible. First, we calculate the deviatoric (true) stress as $\sigma_{T}=\sigma_{i i}-\sigma_{\text {hydr }}$. Subsequently, we convert this to the engineering stress $\sigma=\sigma_{T} / \lambda$. For every configuration the deformation protocol is performed in the $x, y$, and $z$ direction and the output is averaged. Data presented in the manuscript are averages over 4 configurations. If error bars are used, they indicate the standard deviations in the values between these 4 configurations.

\subsubsection{Analysis of chains}

Polymer chains are defined as the set of particles in between crosslinkers, the latter having connectivity different from 2. Some of the $N_{\text {chains }}$ in a network are trivial dangling ends, i.e. one of the ends of the chain has functionality of 1 , and are indicated as $N_{\text {dang. }}$. We also identified (first-order) loops when both chain ends share the same crosslinker, and we indicated these as $N_{\text {loops }}$. Both loops and dangling ends are expected to not contribute to the mechanical response; therefore, in a first approximation, we can define the active chains as $N_{\text {act }}=N_{\text {chains }}-N_{\text {dang }}-N_{\text {loops }}$. We define the chain-length, $L$, as $b *\left(N_{\text {beads }}-1\right)$ where $b=0.96$ is the rest length of the quartic bond and $N_{\text {beads }}$ the number of particles in a polymer chain including the crosslinkers. We define the end-to-end distance $r$ as the Euclidean distance between crosslinkers. To calculate $r$ prior to deformation we use the average crosslinker positions from a simulation run of $10000 \tau$, where the crosslinker locations are saved every $50 \tau$. For the calculation of $r$ during deformation the crosslinker positions are based on snapshots which are saved every $\Delta \lambda=0.01$. In both cases, coordinates are unwrapped to correct for periodic boundary crossings and corrected with respect to their combined centre of mass. We consider a polymer chain to be broken if one of the bonds inside the chain breaks. Breaking of bonds is reported via a custom extension of the LAMMPS code. 


\subsubsection{Affine predictions}

Affine predictions for $r_{i} / L$ distributions and global stress are made based on the time-averaged positions of chain-ends of active chains, i.e., crosslinkers. Based on this configuration we can determine the average location of chain-ends after affine deformation. From these positions we calculate the end-to-end distances of all the polymer chains. Chains are considered broken if their chain-stretch exceeds the maximum stretch of a quartic bond $(1.08 / b=1.129)$. Broken chains are not included in the distributions.

To predict the stress response we assume that the stress response of the single polymers can be described as an extensible freely jointed chain (eFJC) $)^{35}$ which covers both entropic and enthalpic contributions. In this way we can obtain the stress contribution of every polymer based on the location of the chain ends. Combining the contributions of all active polymers, we get our prediction of the virial stress tensor and thus the global response based on affine deformation.

To find the stress contribution of every polymer we rewrite the chain-stretch as $r / L=\frac{r_{L}}{L} \lambda_{b}$, where $r_{L} / L$ is the entropic chain-stretch and $\lambda_{\mathrm{b}}=R_{\mathrm{b}} / b$ the enthalpic stretch of a quartic bond. $\lambda_{\mathrm{b}}$ is found by numerically solving the force balance $\frac{d U_{\text {quartic }}\left(\lambda_{\mathrm{b}}\right)}{d \lambda_{\mathrm{b}}} \lambda_{\mathrm{b}}=k_{\mathrm{B}} T \frac{r}{\lambda_{\mathrm{b}} L} \mathcal{L}^{-1}\left(\frac{r}{\lambda_{\mathrm{b}} L}\right)$ according to Ref. [35], where $\mathcal{L}^{-1}$ is the inverse Langevin equation (we use the approximation by Puso ${ }^{36}$ ). Based on this value we can calculate the force from $F=\frac{k_{\mathrm{B}} T}{b \lambda_{\mathrm{b}}} \mathcal{L}^{-1}\left(\frac{r}{\lambda_{\mathrm{b}} L}\right)$. Note that $k_{\mathrm{B}} T=1.0$ in reduced units.

\subsection{Results and discussion}

\subsubsection{In silico preparation of DNs}

We prepared our networks following the procedure of Refs. [28-30]. In particular, the first network, or sacrificial network, is generated from 10000 particles with a number density $\rho=0.15$ (Figure 6.1(a)). The majority of these particles are bifunctional and can only form linear chains. A fraction $c_{1}=5 \%$ of the particles are tetrafunctional and can crosslink polymer chains. After network generation, we swell the sacrificial network isotropically up to a swelling ratio $\lambda_{0}=L_{\mathrm{box}} / L_{0, \text { box }}$ (Figure 6.1(b)), and we add particles for the second network, or matrix (Figure 6.1(c)). The matrix is formed with a crosslinker fraction $c_{2}=1 \%$ (Figure 6.1(d)). Because $c_{2}\left\langle c_{1}\right.$ the sacrificial polymer chains are shorter $(\langle L\rangle=10.9)$ than the matrix chains $(\langle L\rangle=47.3)$ in line with the empirical design rules for creating tough $\mathrm{DNs}^{9}$. The distribution in chain-lengths is exponential as expected for a random polymerization process (see Section 6.A.2 for details). A detailed description of 

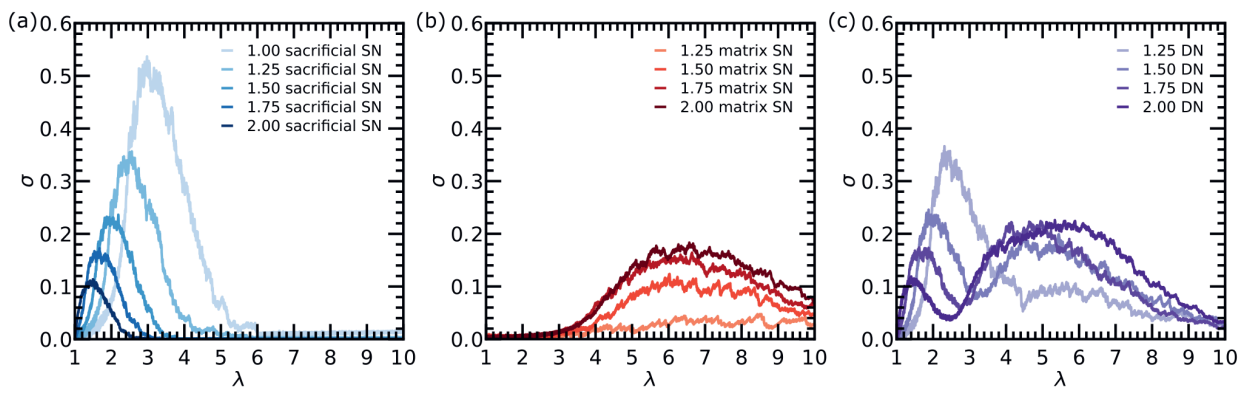

Figure 6.2. Mechanical response of $\mathrm{SNs}$ and $\mathrm{DNs}$ for a range of swelling ratios (see legends). We plot the engineering stress $\sigma$ versus the global stretch $\lambda$ for (a) sacrificial SNs ( $c_{1}=5 \%$ ), (b) Matrix SNs $\left(c_{2}=1 \%\right)$, and (c) DNs $\left(c_{1}=5 \%, c_{2}=1 \%\right)$. Note that the matrix SNs are generated by removing the sacrificial chains from the DNs.

the procedure can be found in Section 6.2.1. We have chosen the parameters based on a trade-off between experimental reality and feasibility of the simulations (see Section 6.A.1 for details).

Note that our protocol for creating DNs differs from other simulation works in several ways. Typically, polymer networks are formed by crosslinking pre-formed chains of given length ${ }^{17,34,37,38}$, rather than using a random-polymerization-like procedure as we do. Furthermore, we prepare DNs via sequential polymerizations, that conceptually resemble the experimental protocols ${ }^{2,3}$, instead of the simultaneous assembly of both networks as previously done ${ }^{17,37,38}$. There are other examples of in silico DNs generated by swelling. However, differently from our procedure, either inter-network crosslinking is allowed ${ }^{33}$, or only the bonds of crosslinkers are allowed to break ${ }^{34}$.

\subsubsection{Mechanical response of in silico DNs}

To obtain the mechanical response of our networks, we perform a uniaxial extension at a constant strain-rate and a constant volume, i.e. we impose a Poisson ratio $v=0.5$. We do this for both the DNs and the stand-alone networks. To facilitate comparison with experimental work, we plot the engineering stress $\sigma$ which is calculated by dividing the deviatoric (true) stress by the global stretch $\lambda$ (see Section 6.2.2 for details). All results are reported in reduced (Lennard-Jones) units.

Following one of the curves in Figure 6.2(a) (e.g. $\lambda_{0}=1.00$ ) we can identify four mechanical regimes. After a short linear response at low strain (the linear elastic regime), the network becomes strain-stiffening, as is expected for entropic springs, around $\lambda=1.50$ (the non-linear elastic regime). Subsequently, strain-softening 
starts from $\lambda=2.25$, induced by breaking of chains, until the maximum strength $\sigma_{\max }$ of the sacrificial $\mathrm{SN}$ is reached at $\lambda=3.35$ (the strain-softening regime). After this point, the stress drops rapidly, indicating that significant damage is done to the network, cracks start to propagate and the capability to carry load is lost (the crack propagation regime). Swelling the networks (without adding the matrix), increases the linear modulus (Figure 6.A.4) and decreases the onset of strain-stiffening, the stretch at maximum strength and the maximum strength. All these effects can be attributed to an increase in the average chain-stretch and are also observed in experiment ${ }^{4,10}$. Note that at the largest swelling ratio $\lambda_{0}=2.00$ a few sacrificial chains break already during the swelling procedure ( $\sim 0.5 \%$ of all chains).

For the stand-alone matrix networks (matrix $\mathrm{SN}$ ), obtained by removing the sacrificial network, we find the same mechanical regimes as for the sacrificial SN (Figure 6.2(b)). However, as on average the matrix chains are longer than the sacrificial chains, the onset of strain stiffening and the maximum stress are found at higher strains. Because the matrix networks are formed after the swelling procedure, we do not find a significant shift in the onset of strain stiffening or the strain at maximum strength with $\lambda_{0}$. We do find a dependency of $\sigma_{\max }$ on $\lambda_{0}$, which is caused by the increase in the matrix monomer density $\rho_{2}$ with the swelling ratio: $\rho_{2}=\rho-\rho_{1}=\rho\left(1-1 / \lambda_{0}^{3}\right)$.

The mechanical response of the DNs (Figure 6.2(c)) is clearly influenced by both the sacrificial network, which dominates at low strain, and the matrix network, which dominates at high strain. The loop in stress at intermediate strains marks the transition between these two regimes. A similar transition is observed for hydrogels and elastomers in experiments in the form of a plateau with a constant stress after a certain yield point ${ }^{4,10}$. This plateau is caused by the separation of the material into a soft and highly stretched region, in which many sacrificial bonds are broken, and a stiff and weakly stretched region, in which the sacrificial network is still intact, also referred to as necking. A force balance between these two regions causes the stress to be constant. We do not observe a plateau here, because our networks are too small to get a separation into a soft and a stiff region. In our simulations the maximum strength of the material $\sigma_{\max }$ is determined by the stress peak either before or after the loop, depending on $\lambda_{0}$. We think this is indicative of the brittle-to-ductile transition which is also a function of $\lambda_{0}$ in experiment ${ }^{4}$. For small $\lambda_{0}$, the first peak is highest, so that the network will fracture completely once the stress exceeds $\sigma_{\max }$, while for higher $\lambda_{0}$, a coexistence between regions of different $\lambda$ is possible at a stress equal to the peak stress of the first network (provided the network is large enough). The brittle-to-ductile transition would then occur at the point where both peaks are of the same height. 
(a)

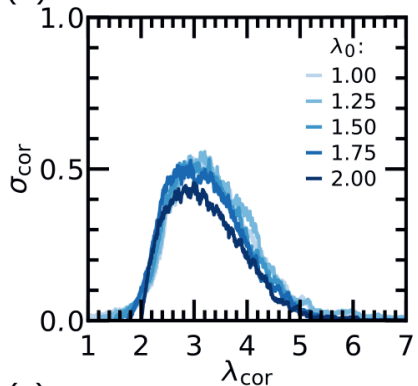

(c)

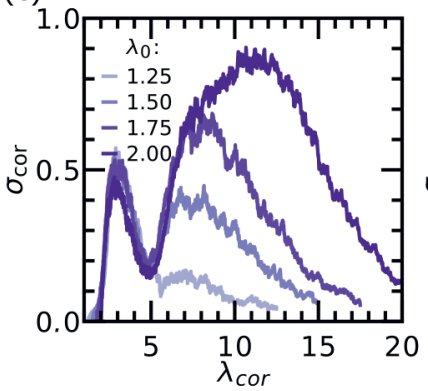

(b)

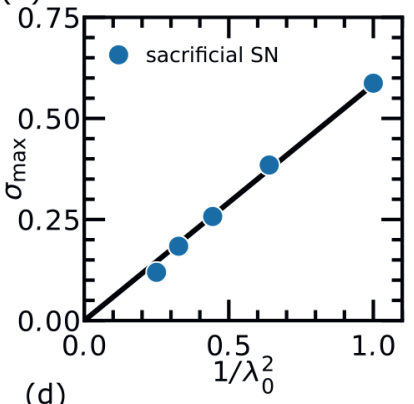

(d)

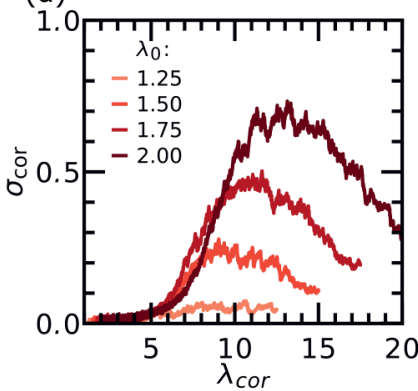

Figure 6.3. The initial stress response is controlled by the sacrificial network. (a) Rescaled stress-strain curves of sacrificial SNs $\left(c_{1}=5 \%\right.$ ), with $\sigma_{\text {cor }}=\sigma \lambda_{0}^{2}$ and $\lambda_{\text {cor }}=\lambda \lambda_{0}$. (b) Maximum stress of sacrificial SN versus $1 / \lambda_{0}^{2}$, a proxy for areal strand density. (c) Rescaled stress-strain curves of DNs ( $\left.c_{1}=5 \%, c_{2}=1 \%\right)$. (d) Rescaled stress-strain curves of the matrix SNs $\left(c_{2}=1 \%\right)$.

We already mentioned that the initial mechanical response of our DNs is dominated by the sacrificial network. Experimental work on hydrogels and swollen elastomers show similar results ${ }^{4,39}$ and reveal that the experimental data can be rescaled onto a single master curve based on the areal strand density of the sacrificial network ${ }^{4,10}$. Figure 6.3 reveals that also our simulation data can be collapsed on a master curve for both sacrificial SNs and DNs, confirming that at least up to the first peak in stress the response is dominated by the sacrificial network. The rescaling corrects for the increase in pre-stretch $\left(\lambda_{\text {cor }}=\lambda \lambda_{0}\right)$ and the reduction in areal strand density in the sacrificial network $\left(\sigma_{\text {cor }}=\sigma \lambda_{0}^{2}\right)$. The onset of strain-stiffening in our rescaled curves occurs around $\lambda_{\text {cor }} \approx 1.5$ and the peak stress falls around $\lambda_{\text {cor }} \approx 3.0$. The latter value is close to the maximum extension limit of our chains under ideal conditions $\left(\lambda_{\text {limit }}=\sqrt{N}=3.37\right.$ with $\left.\langle N\rangle=11.35\right)$.

The collapse for both DNs and sacrificial SNs shows that the maximum stress at the first peak is determined by the strength of the sacrificial network, resulting in the linear scaling between $\sigma_{\max }$ and $\lambda_{0}^{-2}$ shown in Figure 6.3(b). In experiment, a similar scaling was found for the yield stress in ductile $\mathrm{DNs}^{4}$, implying that in 
experiment the yield stress is determined by the strength of the sacrificial network. However, unlike our simulations this linear scaling is only found at high $\lambda_{0}$. At low $\lambda_{0}$, the experimentally measured yield (or breaking) stress increases with $\lambda_{0} \cdot{ }^{4}$ Our explanation for this experimental observation is that the fracture strength of polymer networks is not only determined by the areal strand density, but also by the presence of defects. Because stress concentrates around defects, their presence can drastically reduce the global stress at which macroscopic cracks are formed and global failure is induced. In DNs the effect of these defects in the sacrificial network is mitigated, because the expansion of the defects into macroscopic cracks is hampered by the matrix chains. An increase in $\lambda_{0}$ increases the volume fraction of matrix chains and thus increases the screening effect. As a result, the yield (or breaking) stress will increase with $\lambda_{0}$ as long as global failure is induced by defects in the sacrificial network. Only at high $\lambda_{0}$, when most defects are screened by the matrix chains, the areal strand density will dominate the fracture response, leading to the expected decrease in the yield stress with increasing $\lambda_{0}$. In our simulations we do not observe this sensitivity to defects, because our networks are too small to contain defects that can dominate the fracture response.

On passing, we note that the post-peak response of the simulated networks is more ductile than for experimental ones. This is a finite-size effect, also observed in elastic spring networks ${ }^{40}$. In the remainder, we, therefore, focus on the prepeak behaviour, which we have shown to be consistent with the experimental observations. Furthermore, we note that networks formed at a higher number density of $\rho=0.34$ behave in a similar way to networks formed at $\rho=0.15$ (Figure 6.5).

\subsubsection{Deviations from affine deformation}

The goal of this work is to assess whether the process of inhomogeneous load redistribution affects the global response and the process of damage accumulation at the local level. To quantify this effect, we compare the results of our simulations, where load sharing is intrinsically captured, with an affine prediction for the response, which by definition does not take into account the interaction between the networks. We make these affine predictions based on the evolution of the distribution in end-to-end distances under affine deformation, similar to the statistical damage mechanics models discussed earlier. We assume that the polymers break at an average chain-stretch of $r / L=1.129$, which corresponds to the stretch where the activation barrier for bond rupture is equal to the thermal energy $^{41}$ (see Figure 6.A.5 for details). Note that in our simulations for sacrificial SNs chains break around $r / L=1.08$. We attribute this lower value to the presence 
(a)
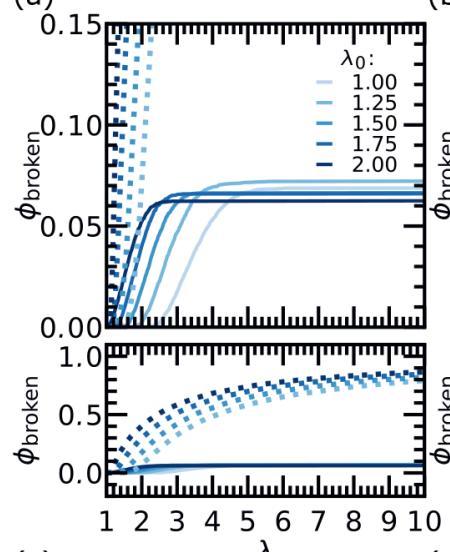

(c)

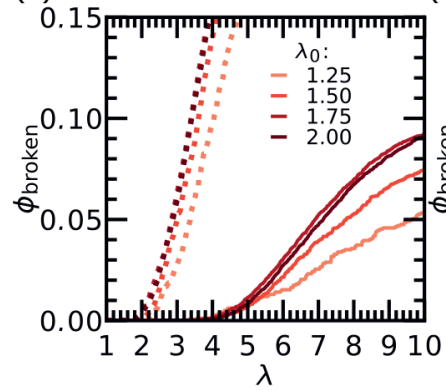

(b)
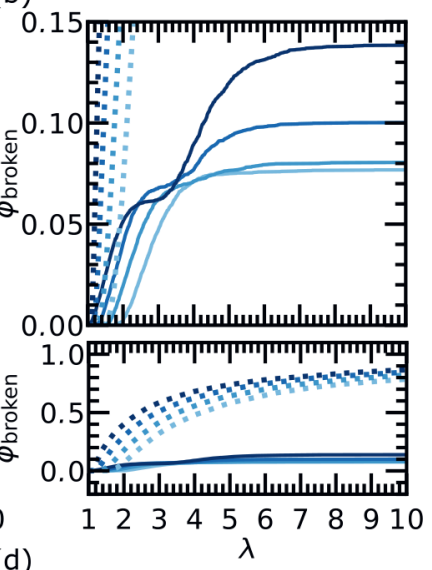

(d)

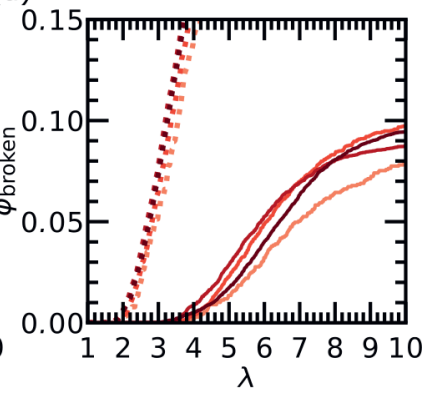

Figure 6.4. Fraction of broken chains $\phi_{\text {broken }}$ as a function of global stretch. The affine prediction for $\phi_{\text {broken }}$ is indicated with the dashed lines. (a) $\phi_{\text {broken }}$ for sacrificial chains in the sacrificial SN $\left(c_{1}=5 \%\right)$ for a range of $\lambda_{0}$ as indicated in the legend. To illustrate the large difference between the affine prediction and the simulations results, we plot the curves for the entire range of $\phi_{\text {broken }}$ in the lower panel and for a smaller range in the upper panel. (b) $\phi_{\text {broken }}$ for sacrificial chains in DNs $\left(c_{1}=5 \%, c_{2}=1 \%\right)$ for the same swelling ratios as (a). (c) $\phi_{\text {broken }}$ for matrix chains in matrix SNs $\left(c_{2}=1 \%\right)$ for a range of $\lambda_{0}$ as indicated in the legend. (d) $\phi_{\text {broken }}$ of matrix chains in DNs $\left(c_{1}=5 \%, c_{2}=1 \%\right)$ for the same swelling ratios as (c).

of topological constraints, which can lead to an underestimation of the chain tension based on the end-to-end distance.

\section{Damage accumulation}

From the microscopic point of view, the main characteristic of the double network response is the enhanced fracture of sacrificial chains. Indeed, we observe a significant increase in the fraction of broken sacrificial chains $\phi_{\text {broken }}$ in the DN (Figure 6.4(b)) compared to the SN (Fig 6.4(a)). The enhancement increases with 
$\lambda_{0}$, reaching up to a $100 \%$ increase for $\lambda_{0}=2.00$. This observed enhancement is a clear indication that in our simulations on DNs the sacrificial network interacts with the matrix network.

Comparing the simulation data (solid lines) with the affine predictions (dashed lines), we find that the affine prediction overestimates $\phi_{\text {broken }}$ by almost an order of magnitude for both the sacrificial SN and sacrificial DN (see bottom panels in Figure 6.4(a) and (b)). In the affine prediction, the behaviour of all chains in a single network is considered to be independent, i.e., if a chain breaks, this has no effect on the stress carried by the neighbouring chains. The significant overestimation of $\phi_{\text {broken }}$ by the affine models, implies that in our simulation interactions at the network level play an important role in the failure response. The simplest way to introduce network structure into the affine model, would be to consider global failure when percolation is lost. However, in such a simple model the fraction of broken chains is still too high with respect to our simulations $\left(\phi_{\text {broken }} \approx 0.99\right)^{42}$. In fact, the fraction of broken chains observed in our simulations is closer to the fractions observed for failure of athermal elastic networks ${ }^{40,43}$, where the fracture response is controlled by network rigidity ${ }^{40,44-46}$.

Going back to our simulation data we also find that the rate of chain failure (the slope of the curves) drops significantly at the start of the transition regime in the stress-strain curve for DNs (Figure 6.3(c)), implying that in a DN the fracture of sacrificial chains takes place in two steps. This is in sharp contrast to the affine prediction where the development of $\phi_{\text {broken }}$ is the same for both the sacrificial $\mathrm{SN}$ and the sacrificial DN, due to the absence of interactions between the two networks.

Combining these insights, we hypothesize that the two-step fracture mechanism in our simulations is controlled by network interactions. The first step is controlled by interaction within the sacrificial network, while the second step is controlled by the topological constraints between the sacrificial network and the matrix. A drop in the fracture rate has been observed experimentally for elastomers ${ }^{4}$ and also experiments on hydrogels identified more than one fracture regime ${ }^{13}$. Finally, we note that in our simulations the rupture of matrix chains does not take place in two steps, however, the fracture of matrix chains occurs earlier in DN networks than in the matrix SN (Figure 6.4(c) and (d)).

\section{Which chains are likely to break?}

A next step in studying the microscopic fracture response is to ask whether we can predict which chains will break. A good first guess would be that shorter chains are likely to break at a lower global strain than longer ones, because for an 

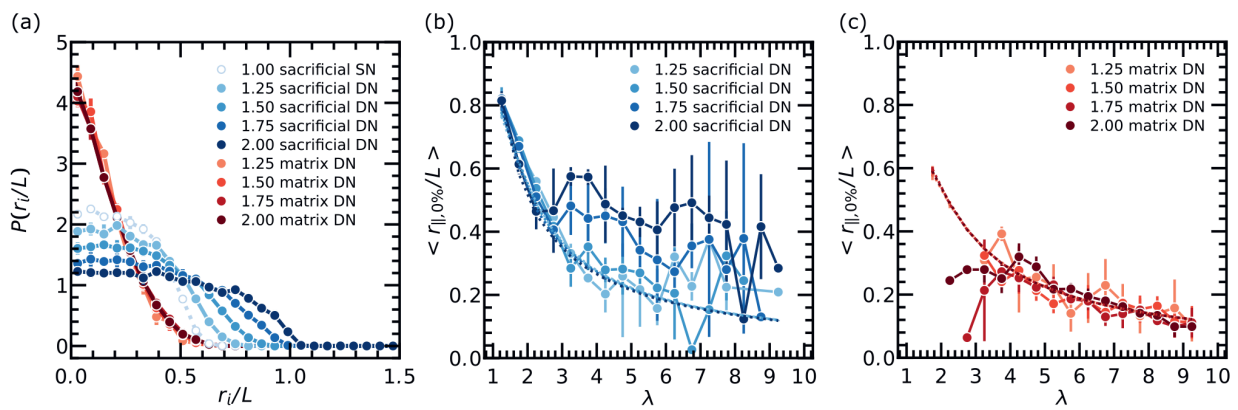

Figure 6.5. Role of initial chain-stretch, or pre-stretch, in chain scission. (a) Chain pre-stretch distribution at $0 \%$ strain for swollen sacrificial networks embedded in a matrix (blue) as well as for matrix networks formed inside swollen sacrificial networks (red). The swelling ratios $\lambda_{0}$ are indicated in the legend. In addition to the data for DNs (solid symbols) the pre-stretch distribution of the sacrificial network prior to swelling $\left(\lambda_{0}=1.00\right)$ is shown (open symbols). Here the stretch $r_{i} / L$ is the end-to-end distance in one direction divided by the contour length of the polymer. The distributions in these plots are based on $r_{i} / L$ in all three dimensions. (b) and (c) Average initial chain-stretch along the direction of applied strain $\left\langle r_{\|, 0 \%} / L\right\rangle$ of broken chains as a function of the global strain $\lambda$ at chain rupture for (b) sacrificial DNs and (c) matrix DNs. The dashed lines represent the affine prediction. In all plots the error bars indicate the standard deviation over 4 configurations.

ideal chain the average stretch at break scales as $N b /(\sqrt{N} b)=\sqrt{N}$. However, we do not find this trend for the sacrificial network in either the simulations results or the affine prediction (Figure 6.A.7). This is because in a network the average end-to-end distance of a polymer is constrained by the connections with other chains in the network. This results in a distribution in the average chain-stretch, or pre-stretch, especially in disordered networks with a distribution in chain-length and local connectivity. In Figure 6.5(a) we show the distribution in pre-stretch as the distribution in $r_{i} / L$, which is the end-to-end distance along one axis, divided by the contour length $L$ of the polymer.

Considering this distribution in average pre-stretch we could hypothesize that instead of the chain-length the chain-stretch at $0 \%$ strain determines when a chain will break, so that the sequence in which bonds break can be predicted from the initial chain-stretch distribution. This is also assumed in the statistical damage mechanics approach ${ }^{25}$. In Figure 6.5(b) and (c) we plot the average pre-stretch at $0 \%$ strain $\left\langle r_{\|, 0 \%} / L\right\rangle$ of broken chains as a function of the global stretch $\lambda$ at which the chains break including both the simulation results (solid lines) and the affine prediction (dashed lines). For the sacrificial network we indeed find that at low strains, the initial chain-stretch does scale with the global strain at break, just as for the affine prediction. For SNs this is true for almost all broken chains (Figure 6.A.7). 
However, in DNs this correlation becomes weaker with an increasing $\lambda_{0}$ and for high $\lambda_{0}$ the correlation even seems to be lost after the first peak stress (this point also corresponds to the minimum in the chain rupture rate). This observation indicates that up to the peak stress the breaking of chains is largely defined by the configuration at $0 \%$ strain. i.e. network rearrangements do not affect the tension on the chains that break before the peak. However, after this peak stress (the start of the transition region in the $\mathrm{DN}$ ) the initial structure no longer controls which sacrificial chains break. As a consequence, the initial chain-stretch is not a predictor of failure anymore and the interactions with the matrix (i.e. topological constraints) dominate. This interpretation aligns with our hypothesis that network fracture takes place in two steps.

Note that the initial chain-stretch distribution of the sacrificial networks is determined by the structure of the network and the level of swelling $\lambda_{0}$. In our simulations the evolution of chain stretch with $\lambda_{0}$ is largely affine with respect to the distribution at $\lambda_{0}=1.00$ (Figure 6.A.3). We also find that the initial chain-stretch distribution of both the sacrificial and matrix networks are the same in the SN and the $\mathrm{DN}$, indicating that prior to deformation, interactions between the networks are negligible. Note that for the matrix polymers we do find a correlation between chain-length and breaking strain in both the simulations results and the affine prediction; this is because there is a wider distribution in chain-lengths in the matrix networks (Figure 6.A.2 and Figure 6.A.6).

\section{Which chains do break?}

The observation that the initial chain-stretch is predictive for when a bond breaks over a large strain-range (Figure 6.5), similar to the affine prediction, is surprising considering the enormous overestimation of broken chains by the affine approximation (Figure 6.4). To investigate what is going on, we plot the distribution of initial chain-stretch for all the intact chains at a particular strain (Figure 6.6). We find that although the initial chain-stretch is predictive for when a chain can break, this does not mean that all chains with that initial chain-stretch will break. Actually, only few of those chains break, which is in sharp contrast with the affine prediction Figure 6.6(b). Our explanation is that in the first failure regime the deformation is largely affine in the undamaged network, but once a polymer breaks, significant stress-relaxation becomes possible via rearrangements at the local level.

\section{Evolution of the pre-stretch distribution}

The data on the accumulation of damage suggest that in our simulations inhomogeneous redistribution of stress is taking place both within single networks during 
(a)

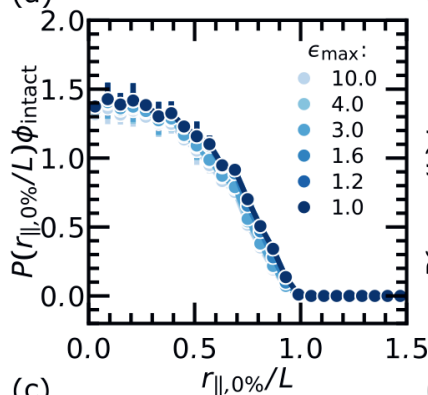

(c)

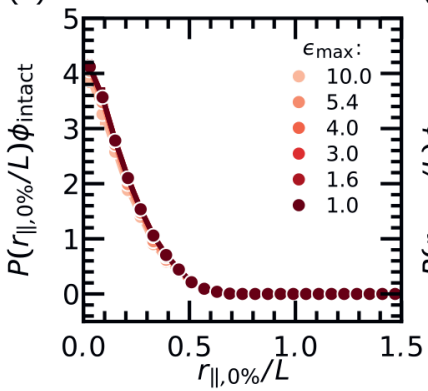

(b)

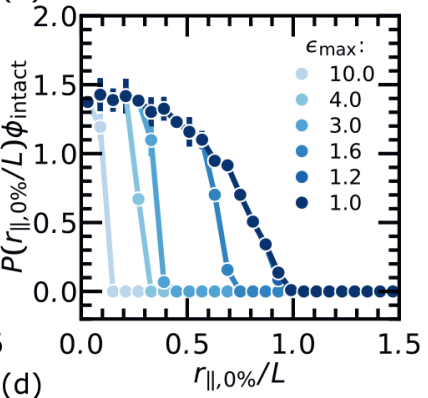

(d)

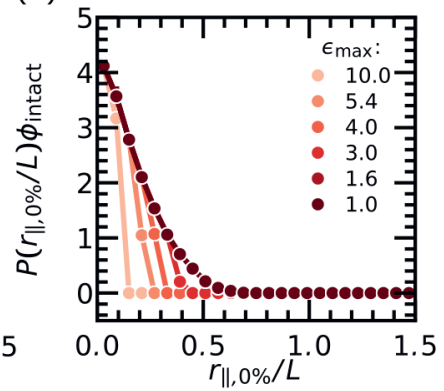

Figure 6.6. Predictability of chain rupture based on initial average chain-stretch. (a) Distribution in initial chain-stretch for all intact sacrificial chains in a DN at $\lambda_{0}=1.75$ at a maximum strain $\epsilon_{\max }$. The distributions are scaled by the fraction of intact chains with respect to the initial configuration (b) Affine prediction for the sacrificial chains in the DN. (c) Distribution in initial chain-stretch for all intact matrix chains in a $\mathrm{DN}$ at a maximum strain $\epsilon_{\max }$. (d) Affine prediction for matrix chains in a DN.

the first failure process and between networks during the second failure process. By analysing the distribution in chain-stretch during deformation we can gain information about the microscopic distribution of stress within the networks and whether this is different in DNs compared to SNs. In particular, we look at the distribution in end-to-end distances parallel to the axis of deformation $r_{\|} / L$ (Figure 6.7).

When the strain is increased from $\lambda=1.0$ to $\lambda=1.2$ for a network at $\lambda_{0}=1.75$ we see that the distribution for both the sacrificial SN and the sacrificial DN flattens, in a similar way as expected for affine deformation (Figure 6.7(a2)). Around the first peak stress at $\lambda=1.6$ (Figure 6.7(a3)) we see an accumulation of chains that are stretched up to their contour length $\left(r_{\|} / L=1.0\right)$ both in the sacrificial SN and sacrificial DN. This behaviour is very different from the response expected based on affine deformation, and is a clear sign of inhomogeneous redistribution of load within the network. 
(a)

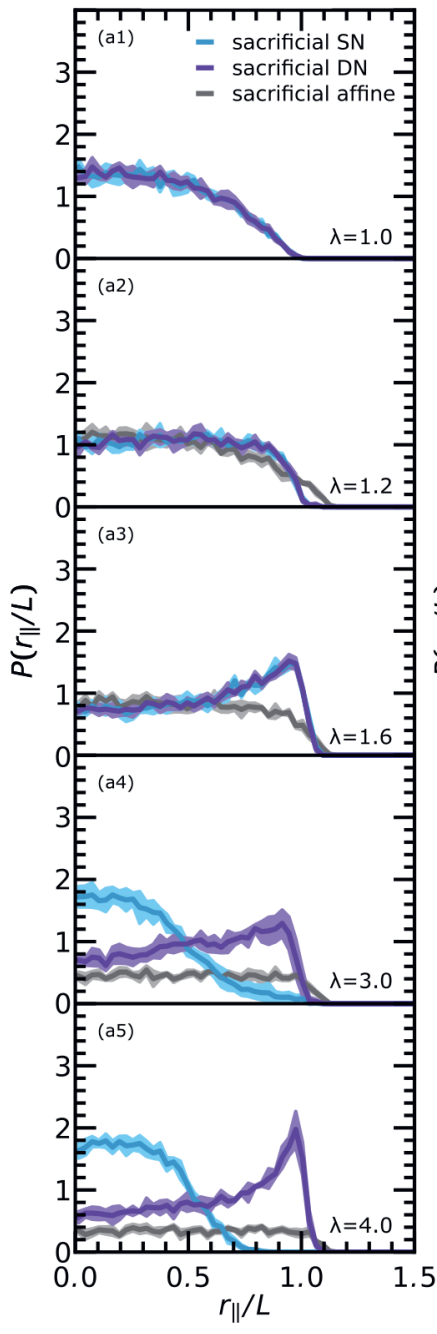

(b)

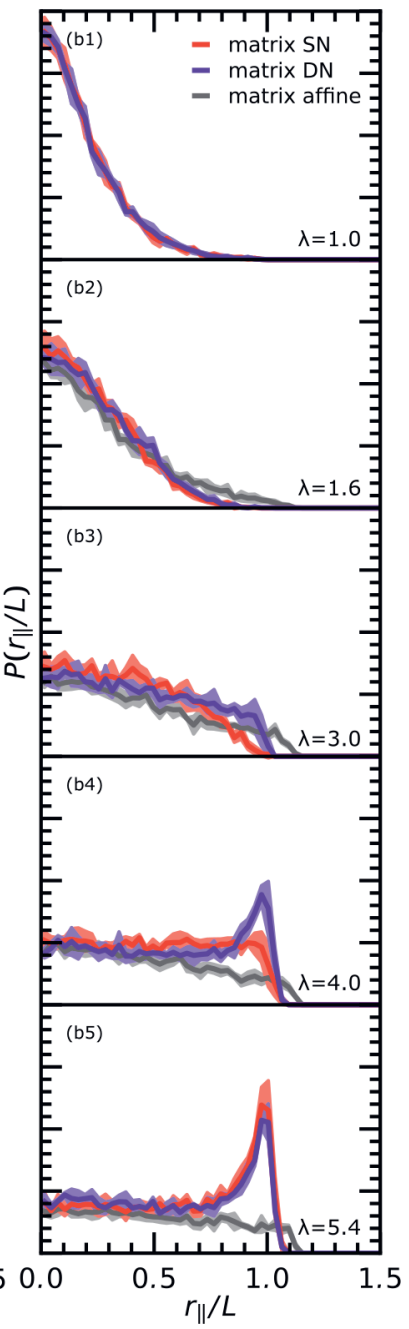

Figure 6.7. Evidence of network-network interaction based on stretch distribution. Comparison of chain-stretch in a network ( $S N$ and $D N$ ) at different strains as indicated in the plot $\left(\lambda_{0}=1.75\right)$. (a) The sacrificial network as stand-alone network (SN, blue) and as part of a DN (purple). (b) Matrix network as a stand-alone network (SN, red) and as part of a DN (purple). For both panels the affine prediction for the chain-stretch distribution is indicated in grey. The shading indicates the standard error computed over the 4 configurations. 
At higher strains $(\lambda=3.0$ and $\lambda=4.0$, Figure 6.7(a4) and (a5)) the behaviour of sacrificial chains in the DN diverges from the behaviour in SNs. While the sacrificial chains in the SN relax to a stretch below the initial chain-stretch and remain there at higher strains, a large part of the sacrificial chains in the DN remain close to their entropic stretching limit $\left(r_{\|} / L=1.0\right)$. At $\lambda=4.0$ (Figure 6.7(a5)) the number of chains at maximum extension increases again in the DN. Far beyond the second peak in stress $(\lambda=10.0)$ the sacrificial chains start to relax, as strongly stretched chains rupture (not shown).

In the matrix network (Figure 6.7(b)) we see that initially the SN and DN behave in the same way, but between a stretch of $\lambda=3$ and $\lambda=4$ we see that there are more stretched chains in the $\mathrm{DN}$ compared to the $\mathrm{SN}$, indicating that during the transition regime an interaction between the two networks arises. These data also match the shift in the stress response we have seen earlier for the matrix DN compared to the matrix SN (Figure 6.2). Overall, it becomes clear that in our simulations we find inhomogeneous stress redistribution at the network level at low strains and inhomogeneous stress redistribution between networks at high strains. These different processes for stress management explain the two distinct failure regimes identified for the sacrificial chains in Figure 6.4.

\section{Impact on global response}

So far we have shown that the microscopic response of the networks is dominated by processes of inhomogeneous stress redistribution, both within single networks and between networks. With increasing strain, these microscopic processes deviate further from the affine picture. The question that remains is: do these processes only matter at the local level or do they also affect the global stress response? To answer this question, we make a prediction for the stress response under affine deformation, based on the pre-stretch distributions shown previously, assuming that the resistance to deformation of a single chain can be described as an extensible freely jointed chain (eFJC, see Section 6.2.4 for details). Although our short chains are not expected to behave exactly as ideal chains, a cross-check of this method with the simulation result reveals that this assumption still serves our purpose (Figure 6.A.6).

Comparing the affine prediction with the simulation results, we find that in the linear regime, the affine prediction agrees quite well with the simulations at low swelling ratios (Figure 6.A.4). For the response at larger strains we look at the networks for $\lambda_{0}=1.75$ in Figure 6.8. We immediately see that in the affine prediction strain-stiffening sets in earlier both for the SNs and the DN. This suggests that in the non-linear elastic regime network rearrangements reduce the 
(a)
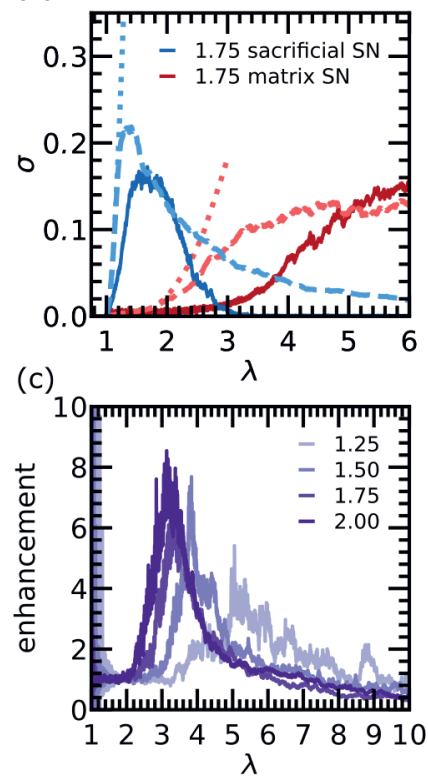

(b)

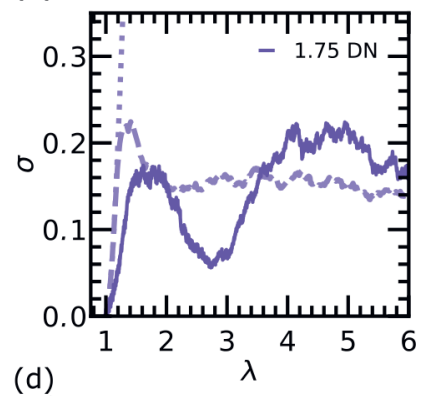

(d)

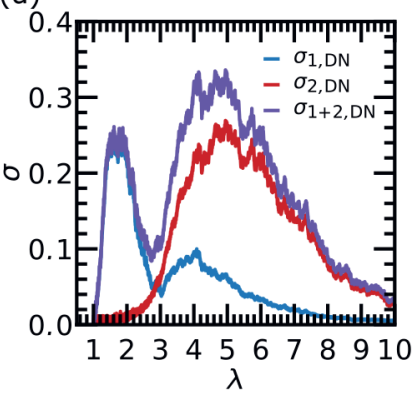

Figure 6.8. The effect of load sharing on the global stress response. (a) and (b) Comparison of the stress response from simulation with the affine prediction $\left(\lambda_{0}=1.75, c_{1}=5 \%, c_{2}=1 \%\right)$. The stress response upon affine deformation is determined from the average crosslinker positions at $0 \%$ strain, assuming that the stress carried by the polymer in between the crosslinkers can be described as a freely-jointed chain with extensible quartic bonds (eFJC, see section 6.2.4 for details). (a) SN response of the sacrificial network (blue) and the matrix network (red) from simulation (solid line), the affine prediction for the mechanical response (dotted line) and the affine prediction considering polymers with $r / L>1.129$ to be broken (dashed line). (b) DN response. (c) Enhancement in measured stress in the DN $\sigma_{1+2, D N} /\left(\sigma_{1, S N}+\sigma_{2, S N}\right)$ with $\sigma_{1+2, D N}$ the measured stress in the DN, $\sigma_{1, S N}$ the measured stress in the sacrificial $\mathrm{SN}$ and $\sigma_{2, S N}$ the measured stress in the matrix SN. Swelling ratios are indicated in the legend. (d) Stress response of the sacrificial network $\sigma_{1, D N}$ (blue) and the matrix network $\sigma_{2, D N}$ (red) embedded in the DN at $\lambda_{0}=1.75$. For reference, we also plot the total stress response of the $\mathrm{DN} \sigma_{1+2, D N}$ (purple).

tension on individual polymers. Even though the impact on the global stress is significant, calculation of the non-affine parameter shows that in most cases these rearrangements are relatively small in the non-linear elastic regime (Figure 6.A.8).

We find that the stress of the affine prediction quickly overshoots the simulation response if bond breaking is not considered. If instead, we assume that chains with a stretch larger than 1.129 break (light-coloured solid lines), we find an SN response that is qualitatively similar to the simulation results. However, in the affine case the onset of strain-softening occurs earlier, the maximum stress is higher, 
the strain at maximum stress is lower and the stress drop after the maximum stress is smoother. These differences imply that inhomogeneous redistribution of load within a network upon breaking of chains has a significant impact on the global stress response. The picture is the same if we compare the affine prediction for the behaviour of the DN with the simulation result. We note that the loop observed in the affine case is less pronounced than in the simulations. We attribute this to the broad distribution in chain-length which causes a relatively smooth decay of the affine stress in the sacrificial network (Figure 6.8(a)) and the relatively small difference in crosslinker density between the sacrificial network and the matrix.

We have found several indications from the microscopic response in our simulations that interactions arise between the two networks if the strain is high enough (Figure 6.4, Figure 6.5,Figure 6.7). To check if these network-network interactions affect the global stress response we compare the stress of the DN with the sum of the stresses for the individual SNs via the enhancement factor $\sigma_{1+2} /\left(\sigma_{1}+\sigma_{2}\right)$ (Figure 6.8(c)). We observe that significant enhancement starts after a certain stretch and that the onset of enhancement decreases as a function of $\lambda_{0}$. The onset of enhancement seems to coincide with the peak strain of the sacrificial SN. After the onset of enhancement, the enhancement factor increases up to a factor of 8.0 at the peak. Also the location of this peak decreases as a function of $\lambda_{0}$. The enhancement peaks just after the end of the transition region. If we plot the stress of the sacrificial network and the matrix network in the DN (Figure 6.8(d)) we see that the enhancement in stress contains contributions of both the sacrificial network and the matrix, further confirming that the enhancement is caused by the interaction between the two networks. The second peak in stress in the sacrificial DN response suggests that even after the yield stress, sacrificial chains resist deformation. Indeed, we find that around this second peak in stress a considerable fraction of the sacrificial chains is stretched up to their contour length (Figure 6.7(a5)).

\subsection{Concluding remarks}

Our simulations confirm that in a DN, both the local and global response is governed by sharing of load at the network level. Similar to experiments ${ }^{4}$, the mechanical response and accumulation of damage at low strains is governed by the sacrificial network even in the strain-softening regime. The behaviour at the microscopic level reveals that upon deformation and damage accumulation stress is redistributed inhomogenously within the network via small and local non-affine rearrangements. 
After the yield stress, the mechanical response is controlled by both the sacrificial network and the matrix network. The enhancement in broken chains (Figure 6.4), the change in failure mechanism (Figure 6.5) and the altered stretch distributions (Figure 6.7) reveal that both networks interact with each other through their topological constraints, leading to large non-affine rearrangements at the network level. These inter-network interactions cause an enhancement in the fraction of broken sacrificial chains, in line with the sacrificial bond principle. At the global level these interactions cause a significant enhancement in the stress response of DNs compared to the SNs (Figure 6.8).

The comparison of our simulation data with affine predictions suggests that for any polymer network (SN or DN) the inhomogeneous redistribution of load through the network can be an important mechanism in the non-linear elastic and fracture response. In the non-linear elastic regime, non-affine rearrangements appear to be small. Therefore, we expect that in this regime the rearrangements are mainly driven by the non-linear stress response of entropic springs in combination with the disordered structure of the network. However, in the fracture regime we find strong deviations from the affine prediction. In particular, we observe that a significant fraction of the chains is stretched beyond their contour length (Figure 6.7). Experiments on single polymer chains also reveal that extension of polymer chains up to this limit is possible ${ }^{41}$. These data suggest that in the fracture regime enthalpic stretching could play an important role in the behaviour of networks prior to the propagation of a macroscopic crack.

Our simulations, provide predictions for the effect of load sharing on the microscopic fracture response. Several of these predictions can be verified in experiment. For example, the low fraction of broken chains (Figure 6.4) could be investigated by quantification of the fraction of broken chains prior to crack propagation by incorporating chain scission reporters in the network such as dioxetane $^{2}$ or anthracene ${ }^{47}$. Our simulations also suggest that the distribution in chain-stretch provides information on the (inhomogeneous) redistribution of load within a network and between networks (Figure 6.5 and Figure 6.7). Although tracking the evolution of the chain-stretch distribution would be a challenging endeavour, experiments on FRET-based force-sensors show that this might be possible experimentally ${ }^{48}$. 


\section{A Appendix}

\section{A.1 Parametrization of the coarse-grained networks}

The input parameters for the network generation process are the particle number density $\rho$, the number of particles $N_{1}$, and the fraction of crosslinkers $c_{1} . \rho$ and $N_{1}$ set the initial box size via $L_{\mathrm{box}}=\sqrt[3]{N_{1} / \rho}$. Our choice for $c_{1}$ is inspired by elastomeric double networks synthesized from polyethyl acrylate (PEA) ${ }^{4}$. For these networks the fraction of crosslinkers $c_{\text {cross }}$ is $1.45 \mathrm{~mol} \%$ relative to the monomer. Because we simulate coarse-grained networks, the particles in our simulation do not represent a single monomer, but a Kuhn length $b$ (which typically encompasses several monomers). To convert $c_{\text {cross }}$ to a $c_{1}$, we used the characteristic ratio $C_{\infty}$ as a coarse-graining factor. Assuming the polymer behaves as a freely jointed chain, $C_{\infty}$ is equal to the number of monomers within a Kuhn length. For PEA $C_{\infty}=9.67$, thus our particle represents 9.67 ethyl acrylate monomers ${ }^{4}$. Using this information we can determine the crosslinker fraction as $c_{1}=c_{\text {cross }} C_{\infty}$, which for PEA gives a crosslinker fraction of approximately $10 \%$. Because such a high $c_{1}$ would result in very short chains, we decided to set $c_{1}=5 \%$.

The parameters for the second network are again inspired by experimental data $^{4,10}$. In our model the variables are $\lambda_{0}, N_{2}$ and $c_{2}$. Because in elastomeric double networks, the sacrificial network is swollen with matrix monomers, $\mathrm{N}_{2}$ is coupled to $N_{1}$ and $\lambda_{0}$. Considering that in an elastomer the monomer density remains constant we can write expressions for the density of the initial network $\rho=\rho_{1 \text {,init }}=\frac{N_{1}}{V}$ and the swollen network $\rho=\rho_{1+2 \text {,new }}=\frac{N_{1}}{V \lambda_{0}^{3}}+\frac{N_{2}}{V \lambda_{0}^{3}}$, with $V$ the volume of the system. By equating these expressions, we find that the ratio between $N_{2}$ and $N_{1}$ can be written as $N_{2} / N_{1}=\lambda_{0}^{3}-1$. Plotting this relation together with experimental data on $\lambda_{0}$ in Figure 6.A.1, we find that this relation approximates the experimental situation for double network elastomers. Although swelling in double network hydrogels can be controlled independently of the monomer concentration, we do observe in at least one article on hydrogels ${ }^{10}$ that $N_{2} / N_{1} \approx \lambda_{0}^{3}-1$.

The fraction of crosslinkers in the second network relative to the number of matrix monomers $c_{\text {cross }, 2}$ is typically a lot lower than in the sacrificial network. For example, in elastomers $c_{\text {cross }, 2}=0.01 \mathrm{~mol} \%$, which would lead to a $c_{2}=$ $c_{\text {cross }, 2} C_{\infty} \approx 0.1 \%$. For our system size it would be improbable to get a percolating second network under those conditions. Therefore, we opt for a $c_{2}$ of $1 \%$. 


\section{A.2 Chain length distribution}

Just like in hydrogels ${ }^{3}$ and elastomers ${ }^{2}$ the polymer chains in our in silico networks have a distribution in chain-length and pre-stretch. Our in silico networks show an exponential distribution in chain length (Figure 6.A.2) for both the sacrificial network and the matrix. This is in accordance with earlier works ${ }^{28,29}$ where it is demonstrated that the chain-length distribution follows asymptotic Flory statistics ${ }^{29}$ as indicated by the grey lines.

Specifically, we create a network from $n_{\text {mon }}=n_{A+B}$ monomers, of which $n_{A}$ monomers and $n_{B}=n_{\text {mon }} c_{x \text {-link }}$ crosslinker. The crosslinkers have a functionality $f_{B}$. If all crosslinkers are used efficiently the number of chain ends $n_{e}=f_{B} n_{B}$ and thus we also know that the number of chains $n_{c}=f_{B} n_{B} / 2$ as all chains have two chain ends. The minimum number of particles in a chain $n_{\min }$ is set by two factors. (i) crosslinkers (B) can only form bonds with monomers (A) such that the smallest chain will always include two crosslinkers and one monomer. (ii) we can add additional particles $n_{\text {add }}$ to every chain after network formation to increase the minimum chain length. If $n_{\text {add }}>0$ we first form a network with $n_{A+B, \text { init }}=n_{A+B}-n_{\text {add }} n_{c}$ and $n_{A}=n_{A+B \text {,init }}-n_{B}$. Please note that the segment length of the polymer $N=l-1$ with $l$ the number of particles in a chain. The number of chains of a certain segment length $n_{N}^{\text {asympt }}$ in this system can be described according to an asymptotic Flory description ${ }^{29}$.

$$
n_{N}^{\text {asympt }}=n_{A}\left(\frac{p_{B}}{p_{A}}\right)^{2}\left(\frac{p_{A}-p_{B}}{p_{A}}\right)^{N-n_{\text {add }}-2}
$$

Here $p_{A}=2 n_{A} /\left(2 n_{A}+f_{B} n_{B}\right)$ and $p_{B}=1-p_{A}$. To get the probability distribution function for chain length, we can write

$$
P_{\text {asympt }}\left(N ; n_{\text {mon }}, c_{\text {x-link }}, f_{B}, n_{\text {add }}\right)=\frac{n_{N}^{\text {asympt }}}{n_{c}}
$$




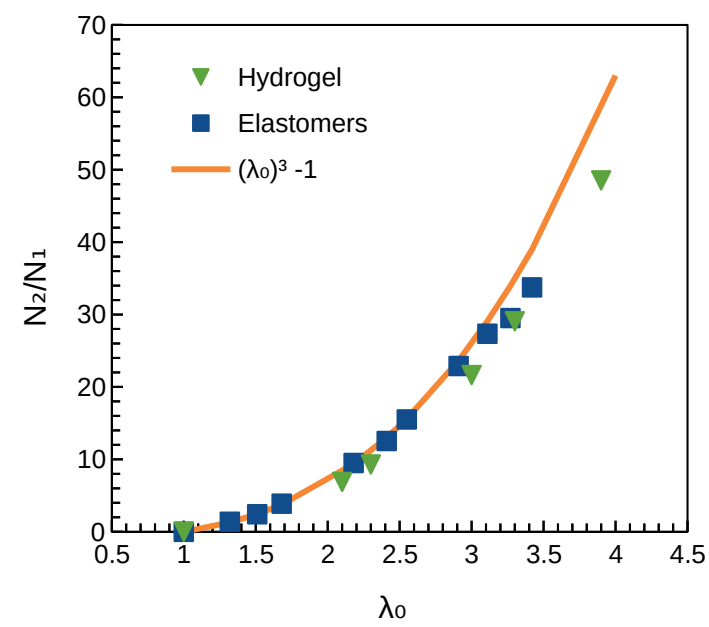

Figure 6.A.1. Fraction of matrix network monomers with respect to sacrificial network monomers as a function of $\lambda_{0}$. The plot show the fractions for experiments on elastomers ${ }^{4}$ and on hydrogels ${ }^{10}$. The orange line is our approximation $N_{2} / N_{1} \approx\left(\lambda_{0}\right)^{3}-1$.

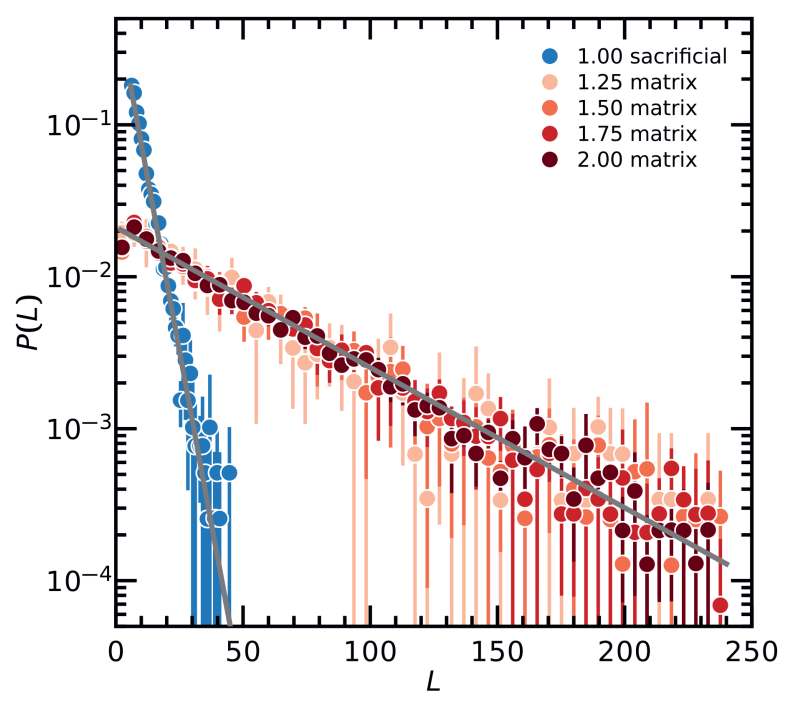

Figure 6.A.2. Chain length distribution within the sacrificial network (blue) and within the matrix networks (red) $\left(\rho=0.15, c_{1}=5 \%, c_{2}=1 \%\right)$. Error bars indicate the standard deviation over 4 configurations. The grey lines indicate the distribution in chain length as expected from asymptotic Flory statistics ${ }^{29}$. 

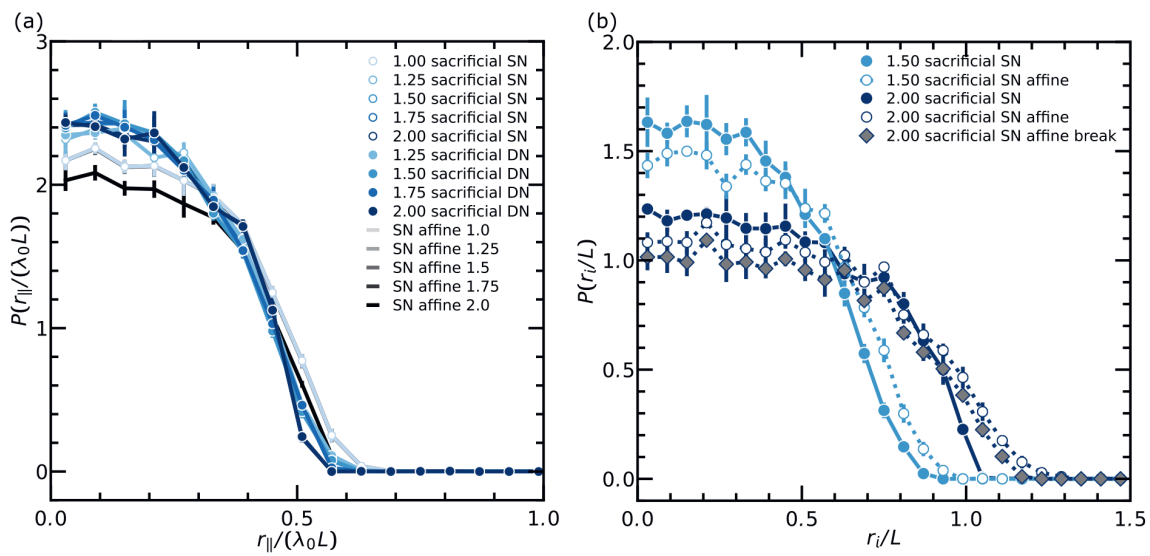

Figure 6.A.3. Evolution of pre-stretch during the swelling procedure. The response to swelling is largely affine. (a) Rescaling of the average chain-stretch distribution of sacrificial chains prior to deformation for SNs (open symbols) and DNs (solid symbols). In addition we show the rescaling of the affine prediction for the average chain-stretch distribution based on affine swelling with respect to the $\lambda_{0}=1.00$ configuration (grey lines). As expected almost all affine predictions collapse on the pre-stretch distribution at $\lambda_{0}=1.00$. Only the affine prediction for $\lambda_{0}=2.00$ does not collapse, due to rupture of polymer chains. (b) Comparison between the average stretch distribution $r_{i} / L$ at $0 \%$ strain obtained from simulation (closed faced circles) and from affine swelling with respect to the average positions at $\lambda_{0}=1.00$ (open-faced circles). For $\lambda_{0}=2.00$ we also show the distribution for affine swelling where polymers that exceed $r / L=1.129$ are considered broken (grey faced diamonds). 
(a)

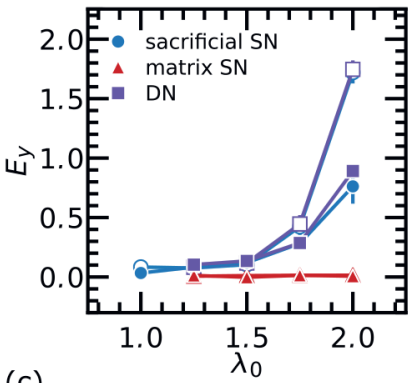

(c)

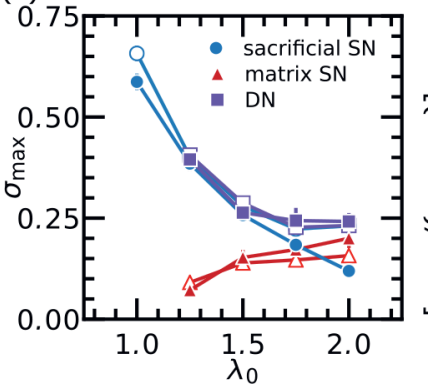

(b)

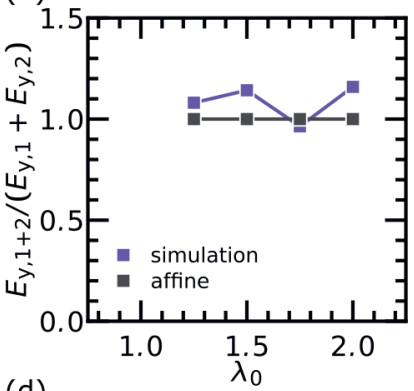

(d)

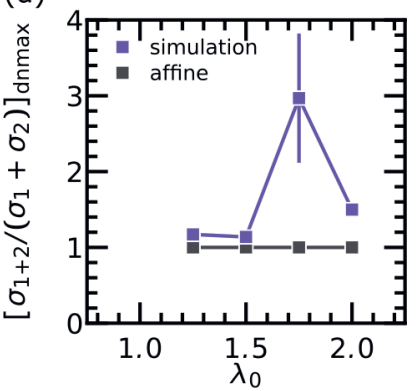

Figure 6.A.4. Enhancement in Young's modulus and peak strength as a function of $\lambda_{0}$. (a) Young's modulus for sacrificial SN (blue), matrix SN (red), and DN (purple) versus the swelling ratio. Including simulation results (closed symbols) and affine predictions (open symbols). Error bars indicate the standard deviation over 4 configurations. To measure Young's modulus in simulation, we apply a step strain protocol. The uniaxial strain is increased in steps $(\Delta \lambda=0.005)$ at a strain rate $\dot{\epsilon}=1 \times 10^{-4}$. Between the strain steps no deformation is applied and the system is equilibrated for $10000 \tau$ (stress is reported every $1 \tau$ ). At each strain step the average stress is calculated based on the final $9500 \tau$ of each step. We calculate the modulus as the slope of a linear fit to the average stress at $\lambda=\{1.0,1.005,1.01\}$. For each configuration the modulus is calculated for deformation in the $x, y$ and $z$ direction and averaged. (b) Enhancement in $E_{y}$ of DNs with respect to SNs for simulations (purple) and affine prediction (grey). (c) Maximum stress $\sigma_{\max }$ for sacrificial SN (blue), matrix SN (red), and DN (purple) versus the swelling ratio. Including simulation results (closed symbols) and affine predictions (open symbols). Error bars indicate the standard deviation over 4 configurations. (d) Enhancement in $\sigma_{\max }$ of DNs with respect to SNs for simulations (purple) and affine prediction (grey). For this panel the $\sigma$ values of the SNs are obtained at the strain at maximum stress of the DN. 
(a)
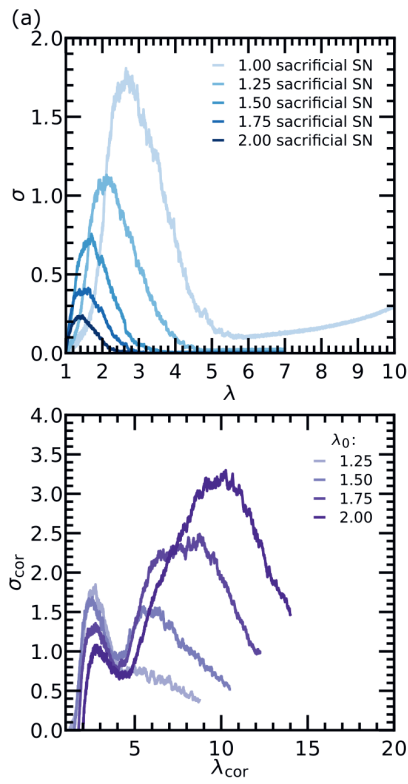

(b)
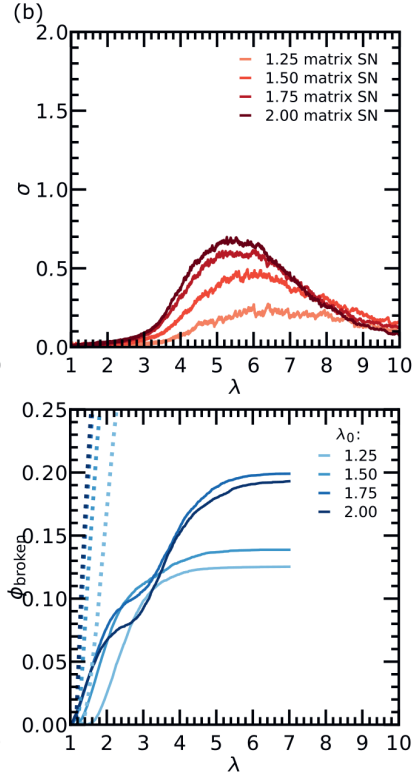

(c)
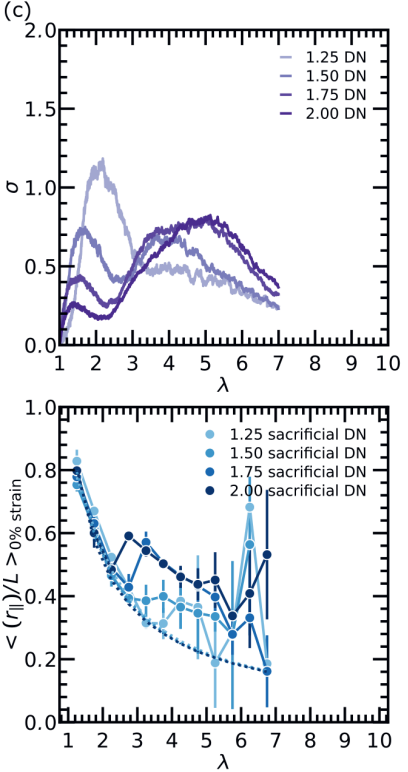

Figure 6.A.5. Mechanical response of networks formed at $\rho=0.34\left(c_{1}=5 \%, c_{2}=1 \%\right)$, instead of $\rho=0.15$ as shown in the main text. Qualitatively similar conclusions can be drawn also in this case. However, we noticed that higher density networks at $c_{1}=5 \%$ accumulate a significant amount of damage if they are swollen beyond $\lambda_{0}=1.25$. Data are averaged over 4 configurations. For every configuration simulations are performed by uniaxial extension in the $x, y$ and $z$ direction and the response is averaged. (a) Stress-strain response for the sacrificial SNs. (b) Stress-strain response for the matrix SNs. (c) Stress-strain response for the DNs. (d) Rescaled DN data. (e) fraction of broken sacrificial chains in simulations on DNs and in the corresponding affine predictions. (f) Average initial pre-stretch of broken sacrificial chains as a function of the global strain for simulations on DNs. 
(a)

(c)
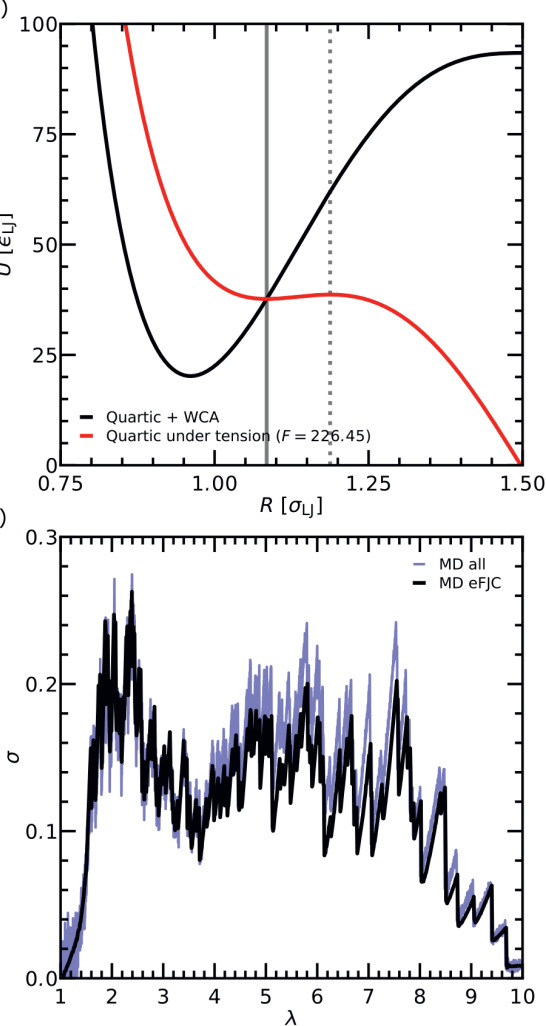

(b)

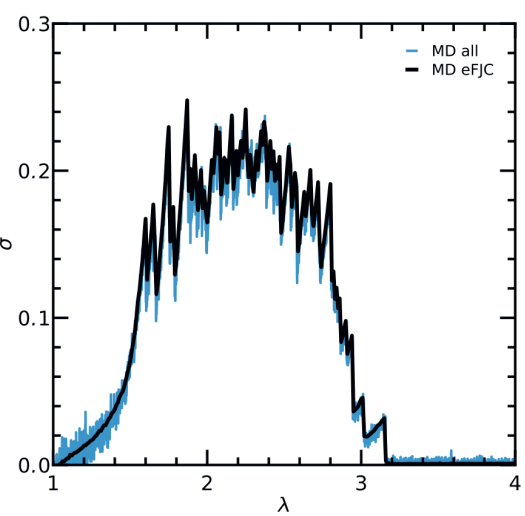

(d)

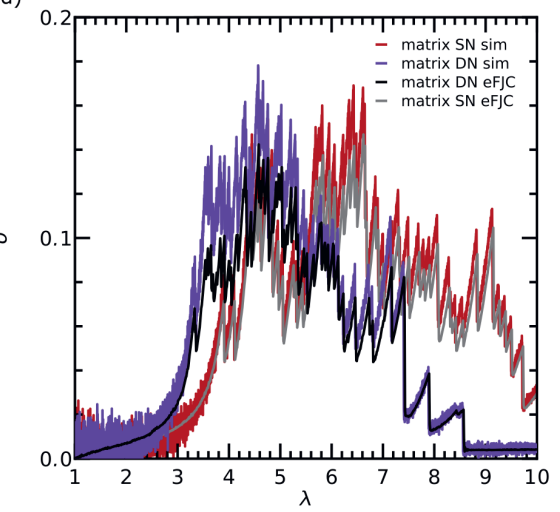

Figure 6.A.6. Estimation of the stress-strain response by mapping the end-to-end distances on extensible freely jointed chains (eFJC). Although our short chains are not expected to behave exactly as ideal chains, our eFJC estimation agrees well with the simulation results. (a) Quartic potential used in our simulations (black), quartic potential with coupled work due to tension $U_{\text {tension }}(R)=U(R)-F \times\left(R-R_{R}\right)$ (red). With $R_{R}$ the rest length under tension (solid grey line). At $F=226.45$ the energy barrier to bond rupture is 1.00 (in reduced units). (b) and (c) Comparison of the stress-strain response in simulation (blue) and the eFJC estimation (black). Data is shown for a single configuration and deformation along the $x$-axis. (b) SN response $\left(\lambda_{0}=1.50, \rho=0.15\right.$, $c_{1}=5 \%$ ) (c) DN response $\left(\lambda_{0}=1.50, \rho=0.15, c_{1}=5 \%, c_{2}=1 \%\right.$ ). (d) For the DN the eFJC approach (slightly) underestimates the stress response, because the end-to-end distance underestimates the tension in chains that are affected by topological constraints. This effect is most prominent for matrix chains, as can be seen in this panel for a network at $\lambda_{0}=1.75$ ( $\rho=0.15, c_{1}=5 \%, c_{2}=1 \%$ ). If we only plot the contribution of the matrix chains to the global stress in a DN, we clearly see that the eFJC estimation (black) underestimates the stress response (red). We also observe that in the matrix SN the difference between the stress response (red) and the eFJC estimation (grey) is smaller, suggesting that the difference in DNs is mainly caused by inter-network constraints. 
(a)

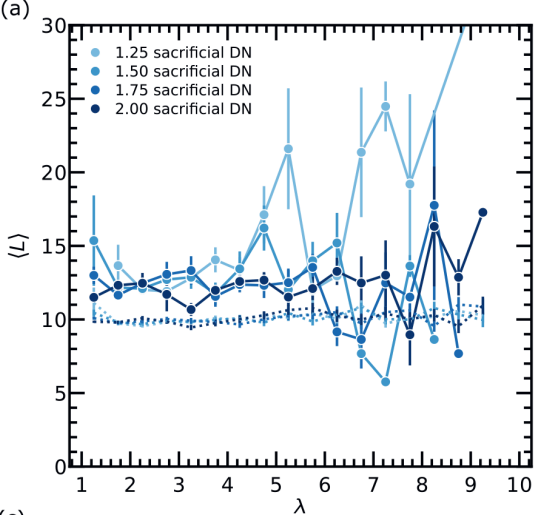

(c)

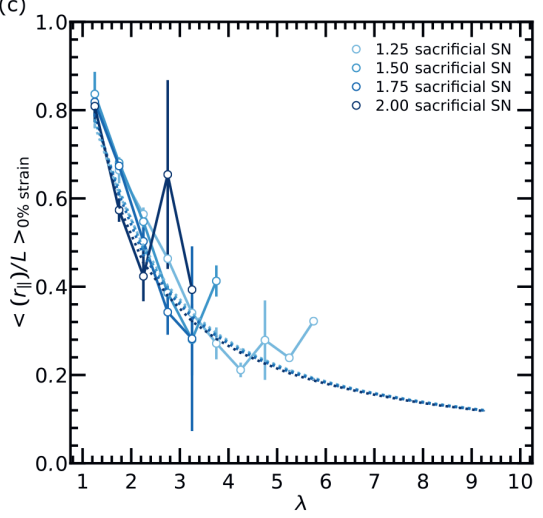

(b)

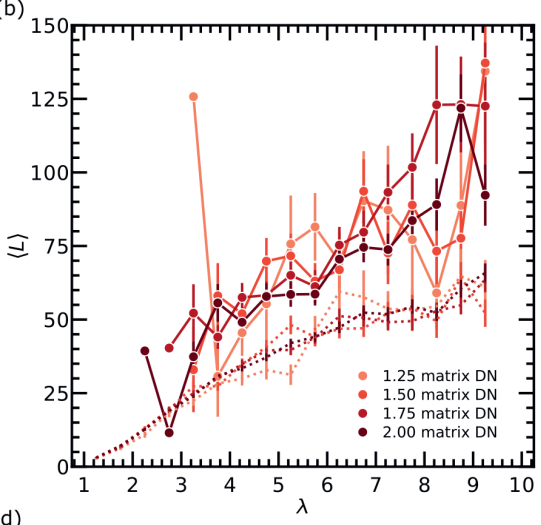

(d)

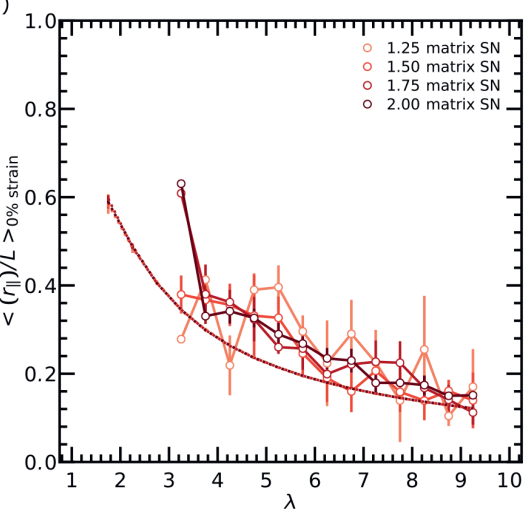

Figure 6.A.7. (a) and (b) Average length of broken chains as a function of global strain found in simulation (solid lines) and according to the affine prediction (dashed lines). (a) Sacrificial chains in DN networks. (b) Matrix chains in DN networks. (c) and (d) Average initial pre-stretch of broken chains as a function of the global strain at break found in simulation (solid lines) and according to the affine prediction (dashed lines). (c) Sacrificial chains in SN networks. (d) Matrix chains in SN networks. 
(a)

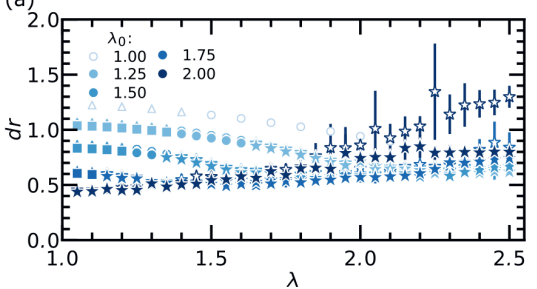

(c)

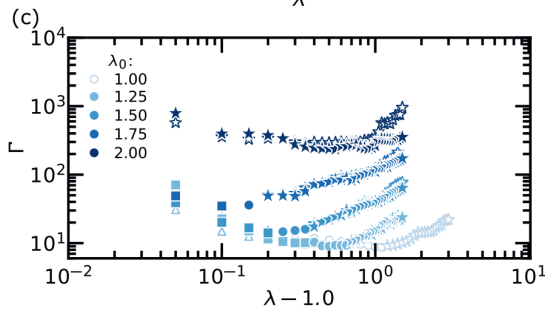

(b)

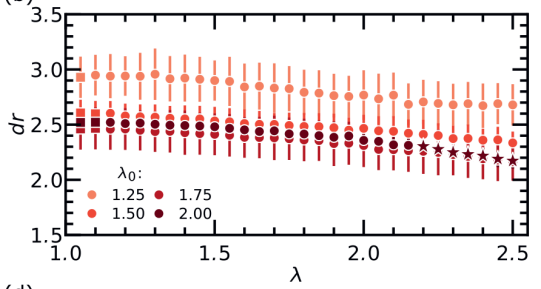

(d)

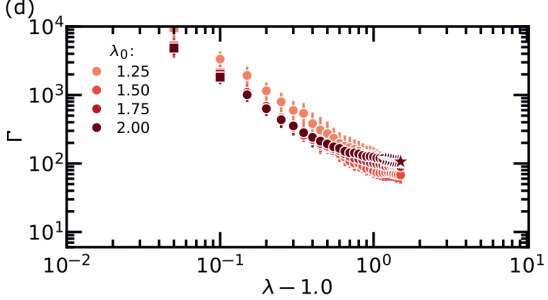

Figure 6.A.8. Effect of $\lambda_{0}$ and uniaxial strain on thermal and non-affine fluctuations of crosslinkers. With $\lambda_{0}$ the size of the thermal fluctuation in the sacrificial network decreases, while the size of the non-affine rearrangements increases. A large part of the non-affine response is either dominated by thermal fluctuations or by rupture of polymer chains. We determine the size of these fluctuations from the crosslinker positions obtained during a step-strain protocol where every strain step $(\Delta \lambda=0.05)$ the system is equilibrated for $10000 \tau$ and crosslinker positions are reported every $50 \tau$. Only crosslinkers that are part of the largest percolating cluster are considered. The particle positions in each snapshot are unwrapped and corrected for the centre of mass (only considering the crosslinkers). Using these coordinates we can calculate the average position of every crosslinker $\langle\boldsymbol{r}\rangle$ and the size of the thermal fluctuations around this position $d r=\left\langle\sqrt{(\boldsymbol{r}-\langle\boldsymbol{r}\rangle)^{2}}\right\rangle$ as a function of strain. In the following plots the marker shape indicates different regimes: around squares the non-affine fluctuations are smaller than the thermal fluctuations, circles represent the elastic regime, and around stars breaking of chains has started. (a) Size of the thermal fluctuations in the sacrificial SNs (open symbols and sacrificial DN (closed symbols) (b) Size of the thermal fluctuations in matrix DNs. (c) and (d) Non-affinity parameter $\Gamma=\left\langle\left(\boldsymbol{u}-\boldsymbol{u}_{\text {aff }}\right)^{2}\right\rangle /\left(\epsilon^{2} \sigma^{2}\right)$. With $\boldsymbol{u}$ the displacement vector of the average crosslinker position $\langle\boldsymbol{r}\rangle$ with respect to $\lambda=1.00$ and $\boldsymbol{u}_{\text {aff }}$ the displacement vector under affine conditions. (c) $\Gamma$ for the sacrificial SNs (open symbols) and sacrificial DN (closed symbols) (d) $\Gamma$ for matrix DNs. 


\section{List of symbols}

\begin{tabular}{|c|c|}
\hline Symbol & Description \\
\hline$b$ & $\begin{array}{l}\text { Polymer segment length or Kuhn length, here defined as the rest } \\
\text { length of a quartic bond }\end{array}$ \\
\hline$c_{\text {cross }}$ & Crosslinker fraction in an experimental system \\
\hline$c_{1}$ & Crosslinker fraction in the sacrificial network \\
\hline$c_{2}$ & Crosslinker fraction in the matrix network \\
\hline$C_{\infty}$ & Characteristic ratio \\
\hline$E_{y}$ & Young's modulus \\
\hline$F$ & Force \\
\hline$k_{\mathrm{B}}$ & Boltzmann constant \\
\hline$L$ & Polymer contour length \\
\hline$L_{\text {box }}$ & Size of the simulation box \\
\hline$L_{\text {box }, 0}$ & Size of the simulation box after NPT-equilibration \\
\hline $\mathcal{L}^{-1}$ & Inverse Langevin equation \\
\hline$N_{1}$ & Number of sacrificial particles or monomers \\
\hline$N_{2}$ & Number of matrix particles or monomers \\
\hline$N_{\text {beads }}$ & Number of particles in a polymer chain, including the crosslinkers \\
\hline$N_{\text {chain }}$ & Total number of polymer chains in the system \\
\hline$N_{\text {dang }}$ & Number of polymer chains with a dangling end \\
\hline$N_{\text {loops }}$ & $\begin{array}{l}\text { Number of chains polymer chains that start and end at the same } \\
\text { particle }\end{array}$ \\
\hline$t_{\text {damp }}$ & Damping time of the Nosé-Hoover thermostat \\
\hline$d t$ & Integration time step \\
\hline$U$ & Potential energy \\
\hline$r$ & Distance between polymer ends \\
\hline$r_{i}$ & Distance between polymer ends along the $i$-th axis \\
\hline$r_{\|}$ & Distance between polymer ends along the axis of deformation \\
\hline$r_{\|, 0 \%}$ & $\begin{array}{l}\text { Distance between polymer ends along the axis of deformation } \\
\text { prior to deformation }\end{array}$ \\
\hline$r_{L}$ & $\begin{array}{l}\text { Corrected distance between polymer ends in the eFJC model, so } \\
\text { that } r_{L} / L \text { can be defined as the entropic chain stretch }\end{array}$ \\
\hline$d r$ & Root mean square size of thermal fluctuations of the nodes \\
\hline$R$ & Interparticle distance \\
\hline$R_{c}$ & Cut-off distance for the interaction potential \\
\hline
\end{tabular}


$\begin{array}{ll}R_{0} & \text { Rest length of a harmonic spring } \\ V & \text { Volume of the simulation box } \\ \Gamma & \text { Non-affinity parameter } \\ \dot{\epsilon} & \text { Strain rate } \\ \epsilon_{\mathrm{LJ}} & \text { Lennard-Jones unit for energy } \\ \epsilon_{\max } & \text { Maximum extension experienced by the network }\end{array}$

$\lambda$

Stretch, defined as the system size along the axis of deformation divided by the initial system size

$\lambda_{0}$

Swelling ratio, defined as the the size of a swollen sacrificial network divided by the size of the unswollen sacrificial network

$\lambda_{b}$

Enthalpic stretch of a quartic bond following the eFJC model

Corrected stretch, taking into account the pre-stretch on sacrificial

$\lambda_{\text {cor }}$

$\lambda_{\text {limit }}$

chains due to swelling

Maximum extension limit of a freely jointed chain under ideal conditions

$v \quad$ Poisson ratio

$\rho \quad$ Number density of particles within the simulation box

$\rho_{1} \quad$ Number density of sacrificial particles within the simulation box

$\rho_{2} \quad$ Number density of matrix particles within the simulation box

$\rho_{\text {init }} \quad$ Number density of patchy particles prior to network formation

$\rho_{\text {initadd }} \quad$ Number density of patchy particles in the system after adding monomers to each self-assembled polymer chain

$\sigma \quad$ Engineering stress

Stress carried by the sacrificial network, the matrix network and $\sigma_{1}, \sigma_{2}, \sigma_{1+2} \quad$ the double network. Sometimes $\mathrm{SN}$ or $\mathrm{DN}$ is added to subscript to clarify whether we are considering the response of a stand-alone network or a network that is part of a double network

$\sigma_{\mathrm{cor}}$

Corrected stress, taking into account the decrease in sacrificial areal chain density with swelling

Strength, defined as the maximum engineering stress that can be

$\sigma_{\max }$

$\sigma_{L J} \quad$ Lennard-Jones unit for distance

$\sigma_{T} \quad$ Deviatoric true stress

$\sigma_{\text {hydr }} \quad$ Hydrostatic component of the true stress

$\tau \quad$ Unit of time in the simulation

$\begin{array}{ll}\phi_{\text {broken }} & \text { Fraction of broken polymer chains with } \\ \text { intact polymers prior to deformation }\end{array}$ 
$\phi_{\text {intact }}$

Fraction of intact polymer chains with respect to the number of intact polymers prior to deformation

\section{References}

[1] J. P. Gong, Y. Katsuyama, T. Kurokawa, Y. Osada, Double-network hydrogels with extremely high mechanical strength. Advanced Materials 15, 1155-1158 (2003).

[2] E. Ducrot, Y. Chen, M. Bulters, R. P. Sijbesma, C. Creton, Toughening elastomers with sacrificial bonds and watching them break. Science 344, 186-189 (2014).

[3] T. Nakajima, H. Sato, Y. Zhao, S. Kawahara, T. Kurokawa, K. Sugahara, J. P. Gong, A universal molecular stent method to toughen any hydrogels based on double network concept. Advanced Functional Materials 22, 4426-4432 (2012).

[4] P. Millereau, E. Ducrot, J. M. Clough, M. E. Wiseman, H. R. Brown, R. P. Sijbesma, C. Creton, Mechanics of elastomeric molecular composites. Proceedings of the National Academy of Sciences of the United States of America 115, 9110-9115 (2018).

[5] Y. Tanaka, A local damage model for anomalous high toughness of double-network gels. Epl 78, 56005 (2007).

[6] H. R. Brown, A model of the fracture of double network gels. Macromolecules 40, 3815-3818 (2007).

[7] H. Xin, S. Z. Saricilar, H. R. Brown, P. G. Whitten, G. M. Spinks, Effect of first network topology on the toughness of double network hydrogels. Macromolecules 46, 6613-6620 (2013).

[8] S. Ahmed, T. Nakajima, T. Kurokawa, M. Anamul Haque, J. P. Gong, Brittle-ductile transition of double network hydrogels: Mechanical balance of two networks as the key factor. Polymer 55, 914-923 (2014).

[9] J. P. Gong, Why are double network hydrogels so tough? Soft Matter 6, 2583-2590 (2010).

[10] T. Matsuda, T. Nakajima, Y. Fukuda, W. Hong, T. Sakai, T. Kurokawa, U. I. Chung, J. P. Gong, Yielding Criteria of Double Network Hydrogels. Macromolecules 49, 1865-1872 (2016).

[11] D. R. King, T. Okumura, R. Takahashi, T. Kurokawa, J. P. Gong, Macroscale Double Networks: Design Criteria for Optimizing Strength and Toughness. ACS Applied Materials and Interfaces 11, 35343-35353 (2019).

[12] T. Nakajima, Generalization of the sacrificial bond principle for gel and elastomer toughening. Polymer Journal 49, 477-485 (2017).

[13] T. Nakajima, T. Kurokawa, S. Ahmed, W. L. Wu, J. P. Gong, Characterization of internal fracture process of double network hydrogels under uniaxial elongation. Soft Matter $\mathbf{9}$, 1955-1966 (2013).

[14] J. Tauber, S. Dussi, J. Van Der Gucht, Microscopic insights into the failure of elastic double networks. Physical Review Materials 4, 063603 (2020). 
[15] R. E. Webber, C. Creton, H. R. Brown, J. P. Gong, Large strain hysterisis and Mullins effect of tough double network hydrogels. Macromolecules 40, 2919-2927 (2007).

[16] T. T. Mai, T. Matsuda, T. Nakajima, J. P. Gong, K. Urayama, Distinctive Characteristics of Internal Fracture in Tough Double Network Hydrogels Revealed by Various Modes of Stretching. Macromolecules 51, 5245-5257 (2018).

[17] Y. Higuchi, K. Saito, T. Sakai, J. P. Gong, M. Kubo, Fracture Process of Double-Network Gels by Coarse-Grained Molecular Dynamics Simulation. Macromolecules 51, 3075-3087 (2018).

[18] Y. Chen, C. Joshua Yeh, Y. Qi, R. Long, C. Creton, From force-responsive molecules to quantifying and mapping stresses in soft materials. Science Advances 6, eaaz5093 (2020).

[19] K. Fukao, T. Nakajima, T. Nonoyama, T. Kurokawa, T. Kawai, J. P. Gong, Effect of Relative Strength of Two Networks on the Internal Fracture Process of Double Network Hydrogels As Revealed by in Situ Small-Angle X-ray Scattering. Macromolecules 53, 1154-1163 (2020).

[20] E. Ducrot, H. Montes, C. Creton, Structure of Tough Multiple Network Elastomers by Small Angle Neutron Scattering. Macromolecules 48, 7945-7952 (2015).

[21] H. M. Van Der Kooij, S. Dussi, G. T. Van De Kerkhof, R. A. Frijns, J. Van Der Gucht, J. Sprakel, Laser Speckle Strain Imaging reveals the origin of delayed fracture in a soft solid. Science Advances 4, eaar1926 (2018).

[22] T. Matsuda, R. Kawakami, T. Nakajima, J. P. Gong, Crack tip field of a double-network gel: Visualization of covalent bond scission through mechanoradical polymerization. Macromolecules 53, 8787-8795 (2020).

[23] X. Zhao, A theory for large deformation and damage of interpenetrating polymer networks. Journal of the Mechanics and Physics of Solids 60, 319-332 (2012).

[24] Y. Liu, H. Zhang, Y. Zheng, A Micromechanically Based Constitutive Model for the Inelastic and Swelling Behaviors in Double Network Hydrogels. Journal of Applied Mechanics, Transactions ASME 83, 021008 (2016).

[25] F. J. Vernerey, R. Brighenti, R. Long, T. Shen, Statistical Damage Mechanics of Polymer Networks. Macromolecules 51, 6609-6622 (2018).

[26] S. R. Lavoie, P. Millereau, C. Creton, R. Long, T. Tang, A continuum model for progressive damage in tough multinetwork elastomers. Journal of the Mechanics and Physics of Solids 125, 523-549 (2019).

[27] V. Morovati, M. A. Saadat, R. Dargazany, Necking of double-network gels: Constitutive modeling with microstructural insight. Physical Review E 102, 062501 (2020).

[28] N. Gnan, L. Rovigatti, M. Bergman, E. Zaccarelli, In Silico Synthesis of Microgel Particles. Macromolecules 50, 8777-8786 (2017).

[29] L. Rovigatti, N. Gnan, E. Zaccarelli, Internal structure and swelling behaviour of in silico microgel particles. Journal of Physics Condensed Matter 30, 044001 (2018). 
[30] V. Sorichetti, A. Ninarello, J. M. Ruiz-Franco, V. Hugouvieux, W. Kob, E. Zaccarelli, L. Rovigatti, Effect of Chain Polydispersity on the Elasticity of Disordered Polymer Networks. Macromolecules 54, 3769-3779 (2021).

[31] S. Plimpton, Fast parallel algorithms for short-range molecular dynamics. Journal of Computational Physics 117, 1-19 (1995).

[32] T. Ge, F. Pierce, D. Perahia, G. S. Grest, M. O. Robbins, Molecular dynamics simulations of polymer welding: Strength from interfacial entanglements. Physical Review Letters 110, 098301 (2013).

[33] W. Wang, Z. Zhang, T. Davris, J. Liu, Y. Gao, L. Zhang, A. V. Lyulin, Simulational insights into the mechanical response of prestretched double network filled elastomers. Soft Matter 13, 8597-8608 (2017).

[34] Y. Yin, N. Bertin, Y. Wang, Z. Bao, W. Cai, Topological origin of strain induced damage of multi-network elastomers by bond breaking. Extreme Mechanics Letters 40, 100883 (2020).

[35] Y. Mao, B. Talamini, L. Anand, Rupture of polymers by chain scission. Extreme Mechanics Letters 13, 17-24 (2017).

[36] R. Jedynak, Approximation of the inverse Langevin function revisited. Rheologica Acta 54, 29-39 (2015).

[37] S. S. Jang, W. A. Goddard, M. Yashar, S. Kalani, Mechanical and transport properties of the poly(ethylene oxide)-poly (acrylic acid) double network hydrogel from molecular dynamic simulations. Journal of Physical Chemistry B 111, 1729-1737 (2007).

[38] H. Li, H. Wu, B. Li, Y. Gao, X. Zhao, L. Zhang, Molecular dynamics simulation of fracture mechanism in the double interpenetrated cross-linked polymer. Polymer 199, 122571 (2020).

[39] T. Nakajima, T. Kurokawa, H. Furukawa, J. P. Gong, Effect of the constituent networks of double-network gels on their mechanical properties and energy dissipation process. Soft Matter 16, 8618-8627 (2020).

[40] S. Dussi, J. Tauber, J. Van Der Gucht, Athermal Fracture of Elastic Networks: How Rigidity Challenges the Unavoidable Size-Induced Brittleness. Physical Review Letters 124, 18002 (2020).

[41] S. Wang, S. Panyukov, M. Rubinstein, S. L. Craig, Quantitative Adjustment to the Molecular Energy Parameter in the Lake-Thomas Theory of Polymer Fracture Energy. Macromolecules 52, 2772-2777 (2019).

[42] Z. Li, Z. Liu, The elongation-criterion for fracture toughness of hydrogels based on percolation model. Journal of Applied Physics 127, 215101 (2020).

[43] S. Deogekar, M. R. Islam, R. C. Picu, Parameters controlling the strength of stochastic fibrous materials. International Journal of Solids and Structures 168, 194-202 (2019). 
[44] M. M. Driscoll, B. G. G. Chen, T. H. Beuman, S. Ulrich, S. R. Nagel, V. Vitelli, The role of rigidity in controlling material failure. Proceedings of the National Academy of Sciences of the United States of America 113, 10813-10817 (2016).

[45] E. Berthier, J. E. Kollmer, S. E. Henkes, K. Liu, J. M. Schwarz, K. E. Daniels, Rigidity percolation control of the brittle-ductile transition in disordered networks. Physical Review Materials 3, 075602 (2019).

[46] J. Tauber, A. R. Kok, J. Van Der Gucht, S. Dussi, The role of temperature in the rigidity-controlled fracture of elastic networks. Soft Matter 16, 9975-9985 (2020).

[47] J. Slootman, V. Waltz, C. J. Yeh, C. Baumann, R. Göstl, J. Comtet, C. Creton, Quantifying Rate-and Temperature-Dependent Molecular Damage in Elastomer Fracture. Physical Review X 10, 41045 (2020).

[48] T. van de Laar, H. Schuurman, P. van der Scheer, J. Maarten van Doorn, J. van der Gucht, J. Sprakel, Light from Within: Sensing Weak Strains and FemtoNewton Forces in Single Molecules. Chem 4, 269-284 (2018). 




\section{Chapter 7}

\section{General discussion}


In this thesis, we explored how soft network materials deal with stress at the microscopic level using a range of coarse-grained network models. We showed that the combination of large extensibility and network disorder, characteristic of soft network materials, dominates both the elastic and the fracture response. In particular, large non-affine rearrangements occur in the network, resulting in a heterogeneous stress distribution. These non-affine rearrangements are of special interest in composite materials, where additional (inter-network) elastic interactions arise from these non-affine rearrangements, leading to a synergistic mechanical response. Despite the variety of our models, we did come across several general trends in their elastic and fracture response, suggesting that also the mechanical response of a wide range of soft network materials, from polymer networks to biological fibre networks to macroscopic networks, can be understood in a general sense.

In Section 7.1.1 we paint a mesoscopic picture of the elastic and fracture response of soft network materials based on the minimal central-force elastic model introduced in Chapter 2. We sketch out future directions for this model in Section 7.1.2 and propose a phase diagram for the fracture response of soft network materials inspired by models for the fracture of stiff heterogeneous materials. Next, we investigate to what extent this minimal description applies to real soft network materials. Additional physical phenomena such as stabilizing fields are considered in Section 7.1.3, additional fracture mechanisms in Section 7.1.4, and inter-network interactions in Section 7.1.5. Realizing the dynamic nature of biological materials and the ever growing interest in transient or self-healing network materials, we introduce a strategy to investigate fracture problems where time is essential in Section 7.2. Subsequently, we discuss whether it is possible to bridge the gap that exists between the mesoscopic picture of fracture in soft network materials and the continuum elastic description of fracture in Section 7.3. Finally, we provide an outlook on the study of fracture in material science and biology in Section 7.4.

\subsection{A mesoscopic picture of elasticity and fracture in soft network materials}

\subsubsection{Damage accumulation in subisostatic networks}

The point of view in all the research presented in this thesis is that the mechanical response of a material emerges from the structure of the network and nonaffine rearrangements at the microscopic level. To capture this microscopic response, we studied an athermal central-force elastic model as a highly coarsegrained representation of soft network materials (see Chapter 2 for details). Below 
(a)

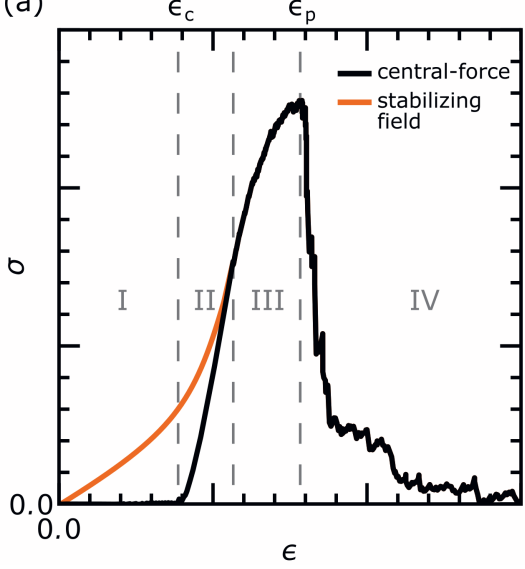

(b)

(c)
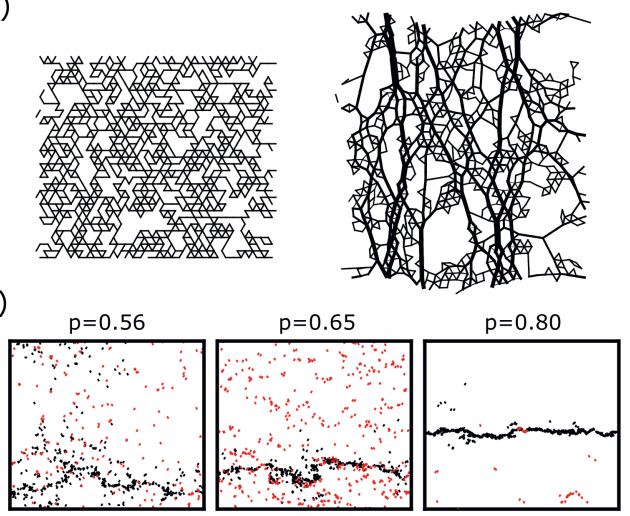

Figure 7.1. The microscopic fracture process in soft network materials. (a) In the global stress versus strain response of subisostatic networks we observe four regimes: linear elastic (I), strain-stiffening (II), strain-softening (III) and stress-drop (IV). Athermal subisostatic central-force networks are completely floppy in regime I (black line). If a stabilizing field is included we do observe a stress response (orange line). (b) The development of force chains after the critical strain $\epsilon_{c}$ leads to an inhomogeneous distribution in stress as indicated by the width of the lines. (c) The microscopic damage response is rigidity dependent. Here we plot the broken bonds with respect to their location in the initial network structure. We distinguish between bonds that break before (red) and after (black) the peak stress. We observe diffuse damage prior to the peak stress, which is maximal for networks around the isostatic point. The shape of the cracks emerging after the peak is more irregular at lower $p$.

we will review the non-linear elasticity and fracture of soft network materials, encompassing elastomers, hydrogels, fibre networks and macroscopic networks, from the view point of this model and present the central-force spring network as a framework for understanding fracture in soft network materials.

\section{Virtually all soft network materials are subisostatic networks.}

Our first assumption in constructing this framework is that virtually any soft network material is subisostatic. This means that the connectivity is so low that based on only central-force interaction the network is not rigid as explained in Chapter 2 (Section 2.3.1). At small deformation stretching of bonds can be prevented by local (non-affine) rearrangements of the network. Only at a critical strain $\epsilon_{c}$ the network acquires rigidity (i.e. becomes resistant to deformation). Beyond this critical strain the network strain-stiffens drastically, due to stretching of bonds ${ }^{1}$. Thus, if we assume that bond scission only occurs after over-stretching of 
bonds, we only expect fracture to occur after this critical strain as demonstrated for minimal network models in literature ${ }^{2}$ and in Chapter 2. In principle, this critical strain is only controlled by the structure of the network. Note that in reality most networks are rigid below this critical strain due to stabilizing fields (see regime I in Figure 7.1), we will come back to this in section 7.1.3.

As the critical strain presents a theoretical lower limit for the occurence of fracture, it would be great if we could estimate this value for real soft network materials. Unfortunatly, there is currently no way to determine an exact value for the critical strain itself based on just a few network structure descriptors, even though the scaling of the (non-)linear elastic response around the critical strain has been found to be surprisingly universal ${ }^{1,3-6}$. For now, if a detailed structure of the network material of interest is known, for example from microscopy on fibre networks, an athermal simulation on a coarse-grained description of the networks does allow an estimation of this critical strain. The question is: What is the appropriate coarse-grained description of such a network material? For athermal fibre networks, this is straightforward: all the material in between crosslinks can be mapped to a (linear) spring with rest length $l_{0}=l_{c}$. However, for (semi-)flexible polymers the contour length of the polymer in between crosslinks is typically longer than $l_{c}$. To correctly estimate the athermal critical strain, this contour length should be captured, for example by introducing multiple segments in between crosslinks with a total length equal to the contour length. In the case of flexible polymers $\left(l_{p} \ll L_{\text {contour }}\right)$ these segments could represent the Kuhn length $b$ (similar to the networks in Chapter 6). Alternatively a rope-like spring could be introduced which only resists extension with $l_{0}=L_{\text {contour. }}{ }^{7}$

\section{The stress distribution in subisostatic networks is heterogeneous beyond the critical strain.}

In any network material that only starts to break after this athermal critical strain, fracture will be dominated by enthalpic stretching. When stretching these networks beyond their critical strain, a highly inhomogeneous (exponential) distribution in stress arises at the level of bonds as we have observed in Chapter 2 (Section 2.3.1) and Chapter 3 (Section 3.3.6). Furthermore, we think that the peak in the stretch distribution of the polymer chains in Chapter 6 (Figure 6.7) is a reflection of this exponential distribution at the bond level. This emerging distribution is associated with the development of force chains, paths where stress concentrates within the network, similar to the structures observed in granular packings ${ }^{8-11}$. Judging from the images in Figure 7.1(b) these force chains are connected to each other, therefore, it might be more accurate to call these structures force networks. With an increase 
in strain, more bonds get stretched, resulting in distinct strain-stiffening at the global level and a wider stretch distribution at the network level. In Chapter 3 (Figure 3.8) we showed that the evolution of this distribution can be quantified by following its higher moments. In particular, we found that the skewness and the kurtosis grow prior to fracture. A similar trend was found for the chain stretch distributions in Chapter 6 (not shown). As stretch distributions are measurable in simulations and in some experiments, these provide a great opportunity for experimental verification of inhomogeneous stress.

\section{Stress redistribution at the network level rather than stress concentration con- trols damage accumulation prior to the peak stress.}

So far we have looked into the reversibly elastic response of soft network materials (regime II in Figure 7.1(a)). We can also study their fracture response (regime III and IV in Figure 7.1(a)) from the perspective of our athermal central-force elastic model, if we assume that individual bonds break in a brittle fashion if they exceed a rupture threshold $\lambda$. For experimental systems $\lambda$ can be estimated from experiment ${ }^{12,13}$ or theory ${ }^{14}$. Note that for polymer networks we proposed to coarse-grain up to the Kuhn length. In that case, $\lambda$ is the relative extension of the atomistic bonds (covalent or non-covalent) in the polymer backbone and not the extensibility of the polymer chains with respect to their rest length. This approach allows a prediction for the (athermal) fracture strength of any network. Because the estimated athermal fracture strength considers damage accumulation in the strain-softening regime and the inhomogeneous distribution of stress, this prediction would be more accurate than an estimate of strength based on the areal strand density, which has been used previously for polymer networks ${ }^{15,16}$. In addition, this analysis provides an estimation for the extensibility via the fracture strain $\epsilon_{p}$.

Our simulations in Chapter 2 (Figure 2.8) and Chapter 3 (Figure 3.7) indicate that the peak stress and the peak strain depend on network size. However, the fracture response up to the peak stress is barely affected by the size of the system. Therefore, our central-force elastic network models seem to be ideally suited to study the accumulation of damage in soft network materials prior to the nucleation and propagation of cracks (see Section 7.1.2 for a discussion on nucleation and propagation). During the strain-softening regime we see a surprising development in the stretch distribution. At the first point of bond breakage the kurtosis and skewness sharply decrease, until a minimum is reached that coincides exactly with the peak stress. This is in contrast to the affine behaviour of the networks described in Chapter 6, where the higher moments keep increasing in the fracture 
regime (not shown). This behaviour reveals that the accumulation of damage has a strong influence on the distribution of stress within the network and suggests that redistribution of stress after bond rupture plays an important role.

For subisostatic networks the damage pattern up to the peak stress is highly diffuse (Figure 7.1(a)) and spreads throughout the entire network, similar to observations for elastomers ${ }^{16}$. Since diffuse damage takes place in the bulk of the material it can have a significant impact on the global response. In particular, the diffuse damage allows the dissipation of elastic energy as demonstrated by the Mullins effect in double networks ${ }^{17}$. The diffuse character of the damage pattern suggests that stress concentration around some sort of defect does not play a significant role in most of the strain-softening regime of subisostatic athermal networks and that instead the emerging force networks control where bonds break. Diffuse damage is also observed for hyperstatic spring networks with a very broad distribution in $\lambda$. For those networks the damage evolution is completely uncorrelated in the initial stage ${ }^{18}$, however, because in our case $\lambda$ is the same for each element and the network structure controls where the force chains occur, we can not rule out that some spatial or temporal correlation exists in our central-force elastic model. A study of the evolution in the accumulated damage and in the void size distribution (see Chapter 5 for an example), could provide further confirmation that stress concentration does not play a major role in the strain-softening regime. A connected question is whether damage accumulation does become (strongly) correlated close to the peak stress, shifting from diffuse damage to crack nucleation. If so, this would provide insight in the origin of crack nucleation and allow forecasting of the transition from regime III to regime IV, i.e. the point where a material can no longer carry additional stress.

\subsubsection{Future questions regarding the central-force elastic model}

Even though the central-force elastic model presented above is a highly simplified representation of soft network materials, we have shown that it can be used as an effective tool to study and understand their fracture response. However, there are still several open questions regarding the microscopic fracture mechanism in this model, especially beyond the peak strain. A further exploration of this model is required to answer these questions.

\section{Network topology and force networks}

The scaling of fracture properties with network connectivity shows there is a strong relation between the structure of a network and how it breaks. One particular question that is asked in literature is whether the location of damage can be 
forecasted based on the network topology. In Ref. [19] it has been demonstrated that a purely topological measure, the geodesic edge betweenness centrality, is a good predictor for bond fracture in subisostatic, but very stiff, networks. This method is based on the detection of shortest paths in the network, the set of shortest paths that connect any possible combination of two nodes. Geodesic edge betweenness centrality measures how often a bond in the network is visited by these shortest paths in the network. Ref. [19] shows that a bond that is part of many shortest paths is more likely to fail. In order to get a structural understanding of how fracture is controlled it would be interesting to test the predictive power of geodesic edge betweenness centrality for the central-force elastic model, especially for highly deformable networks.

However, we expect that an even better understanding of the fracture response can be obtained by studying the structure of the force network. In the centralforce elastic model the fracture of bonds is preceded by the emergence of force networks. By eye there does not seem to be a one-to-one correspondence between the structure of these force networks and the distribution of voids in the diluted network, especially below the isostatic point. It, therefore, seems likely that in diluted spring networks the accumulation of damage and the approach to the peak stress is controlled by the evolution of the force networks instead of the initial distribution and evolution of voids in the network. In order to test this hypothesis it would be helpful to identify force networks and quantify their structure. One straightforward method, also used for granular packings, is to analyze the distribution in the bond tensions that we find after energy minimization at a particular strain ${ }^{9}$ or by analyzing the structure of connected bonds above a certain force threshold ${ }^{10}$. Another possible route is the identification of states of self-stress. Each state of self stress represents a set of bond tensions that results in mechanical equilibrium (i.e. the sum of forces at each node is zero). For granular packings it is argued that the force chains are linear combinations of these states of self-stress ${ }^{20,21}$. Just looking at the number of states of self-stress as a function of stress can already be insightful. In Ref. [22] it was demonstrated that in a Mikado network the onset of rigidity (i.e. at the critical strain) a single state-of-self stress emerges that is responsible for the rigidity transition. This observation could be very relevant in terms of fracture, because for a sufficiently small $\lambda$, rupture of the first bond will be controlled by this emerging state of self-stress. What is not known is what happens after the bond breaks. Are entirely new states of self-stress formed, or does it remain very similar to the old state? How do states of self-stress develop towards the peak stress? Is it possible that at high thresholds there are multiple states of self-stress? Studying the evolution of the states of self-stress could thus be used to study the strong $\lambda$-dependence around the isostatic point. 


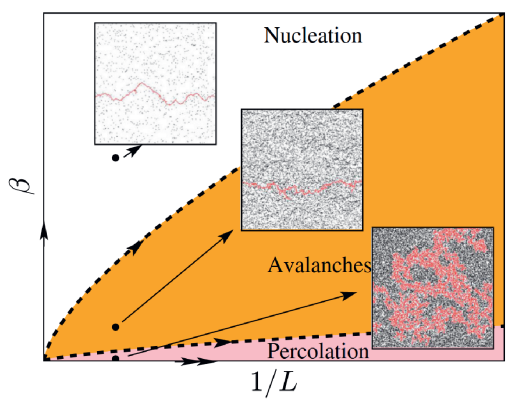

Figure 7.2. Phase diagram for the size dependent fracture response of random fuse networks by Shekhawat et al. ${ }^{23}$ The diagram distinghuises three fracture regimes: percolation, avalanches and nucleation. Which of these fracture responses will occur depends on the inverse network size $1 / L$ and the inverse disorder $\beta$. The images show the system spanning damage cluster (red) and the broken bonds that are not part of this cluster (black). Reprinted with permission from Ref. [23]. Copyright 2013 by the American Physical Society.

\section{Rigidity, disorder, and system size}

Up to the peak stress, the global stress response in the central-force elastic model is almost independent of network size, implying that at the microscopic level the elastic and fracture response are not altered by system size. As far as we have seen, damage accumulation in this regime has a diffuse character (Figure 2.9 or Figure 3.6). At the peak, a size effect is observed in the fracture stress $\sigma_{p}$ and fracture strain $\epsilon_{p}$ in the form of a monotonic decrease with system size; this phenomenon might be associated with crack nucleation and requires more investigation, for example via the procedures proposed in Section 7.1.2. In contrast, the global stress response after the peak stress, is highly non-monotonic as a function of network size and changes smoothly from a force-chain dominated response to a stress concentration dominated response around a characteristic size $L^{*}$ as discussed in Section 2.3.3.

This observation shows similarities with the crossover from fracture via a percolating damage cluster to fracture via a system spanning crack observed in literature ${ }^{2,23-26}$. A study of this cross-over in random fuse networks by Shekhawat et al. ${ }^{23}$ resulted in the phase diagram in Figure 6.8. The study shows that the fracture response of a fuse network can change from damage percolation to crack nucleation by an increase in system size (here the inverse system size $1 / L$ is plotted) or by a decrease in disorder (here the inverse disorder $\beta$ is plotted), in this case the distribution in rupture thresholds. Furthermore, a region characterized by avalanches is observed in between these fracture regimes. Driscoll et al. ${ }^{26}$ observed similar behaviour for elastic networks. However, they also demonstrated 
(a)

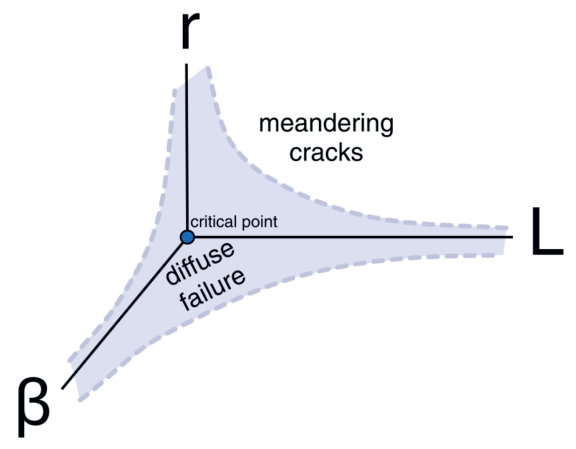

(b)

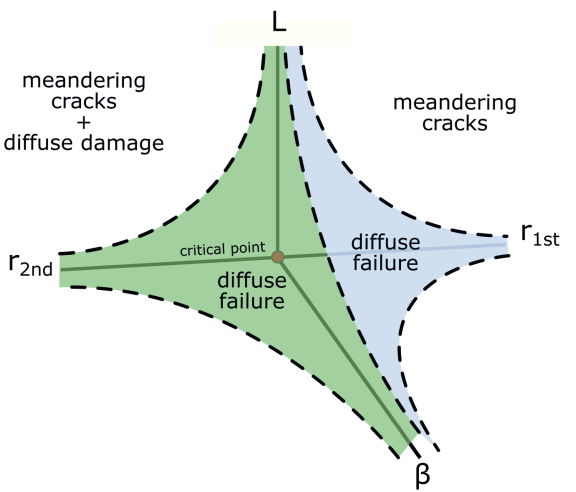

Figure 7.3. (a) Phase diagram as a function of system size $L$, rigidity $r$ and inverse disorder $\beta$ proposed by Driscoll et al. ${ }^{26}$ based on experiments and simulations on elastic networks. Close to the critical point the material fracture occurs by the formation of a percolating damage cluster, while far from the critical point the fracture occurs by the formation of a system spanning meandering crack. In between these limits a cross-over occurs at a characteristic size, rigidity or disorder. (b) Our proposed phase diagram for fracture regimes of central-force elastic networks. In addition to (a) we distinguish between first order and second order rigidity.

the existence of a cross-over as a function of the rigidity $r$ at fixed system size and disorder, adding an additional axis to the phase diagram from Figure 7.2 as shown in Figure 7.3(a). In particular, it was demonstrated that for twodimensional hyperstatic networks a crossover occurs from crack nucleation to damage percolation if the network connectivity approaches the isostatic point. The emerging picture is that a cross-over can be expected depending on the distance from a critical point, with fracture by crack nucleation if any of these values is large $(x \rightarrow \infty)$ and fracture by damage percolation (diffuse failure) if one of these values is really small $(x \rightarrow 0)$.

If our observations for the post-peak response are indeed related to this crossover behaviour in the evolution of microscopic damage after the peak stress, we can draw a similar phase diagram for the fracture response of the central-force elastic model as shown in Figure 7.3(b) as a function of $r, \beta$ and $L$. We note that in our study it seems $r$ does not only depend on the distance from the isostatic point, but also on $\lambda$, thus we propose that $r\left(p-p_{c}, \lambda\right)$. Furthermore, we distinguish between first-order rigidity $r_{1 s t}$ and second-order rigidity $r_{2 \text { nd }}$, considering the different origins of the elastic response leading up to fracture for hyperstatic $\left(p>p_{c}\right)$ and subisostatic networks $\left(p<p_{c}\right)$. Finally, we assume that also for subisostatic networks the fracture response depends on the distance from the isostatic point $\left|p-p_{c}\right|$. A quantitative study of the fracture response in both regime 
III and regime IV is required to establish to what extent the microscopic fracture response of subisostatic networks differs from hyperstatic networks. Are distinct fracture patterns observed (i.e. a qualitative difference), as was initially suggested by Zhang et al. ${ }^{2}$ or can all the descriptors of the microscopic fracture response be rescaled onto a single master curve (i.e. a quantitative difference), just like the fracture abruptness in the central-force elastic model? A more detailed study of the spatial damage distribution (see Figure 7.1(c)), for example by monitoring the evolution of the crack width ${ }^{26}$ or avalanche size ${ }^{23}$, will be required to answer this question.

To conclude, the proposed fracture diagram also contains an axis describing the effect of disorder. This concerns the disorder in the elements, as opposed to the network disorder captured by $r$. In our case this could be the width of the distribution in $\mu, l_{c}$ and/or $\lambda$ (e.g. $\delta \lambda$ in Chapter 5). For random fuse networks 27,28 and random spring networks ${ }^{18,29,30}$ disorder in the rupture threshold has been studied in great detail especially for very wide distributions in $\lambda$, in order to model fracture in stiff heterogeneous materials like concrete. In this thesis we have only explored the $\beta$-axis for undiluted triangular networks in Chapter 5; however, we did not explore the fracture response in the $\left(\beta, r_{2 n d}\right)$-plane. We expect that compared to the rigidity axis, the disorder (in $\lambda$ ) is not very relevant for polymer networks. For fibre networks and metamaterials, the distribution will be wider due to the variations in fibre dimensions, however, still comparatively narrow with respect to the distributions explored for stiff materials. Nevertheless an exploration along this axis could provide insight into the debate whether disorder should be avoided $^{31}$ or embraced $^{32}$ in the design of strong and tough materials.

\subsubsection{The role of stabilizing fields}

\section{The linear response of real soft network materials is controlled by a stabilizing field.}

So far we assumed that after the critical strain the elastic response is caused purely by enthalpic stretching. However, it should be considered that in reality soft network materials are influenced by a stabilizing field, such as bending rigidity (Chapter 4), thermal fluctuations (Chapter 3) or a soft matrix (Chapter 4). Other methods of stabilizing the network are by pre-stressing the network. This can either be done using active components ${ }^{33,34}$ or by stretching the network ${ }^{7,35}$. From literature ${ }^{3}$ and our own work (Figure 3.3 and Figure 4.A.10) we already know that these stabilizing fields alter the rigidity transition in a coarse-grained network model. At the global level that means there is now a smooth, but often highly non-linear transition from the stabilized to the stretching regime (regime I and 

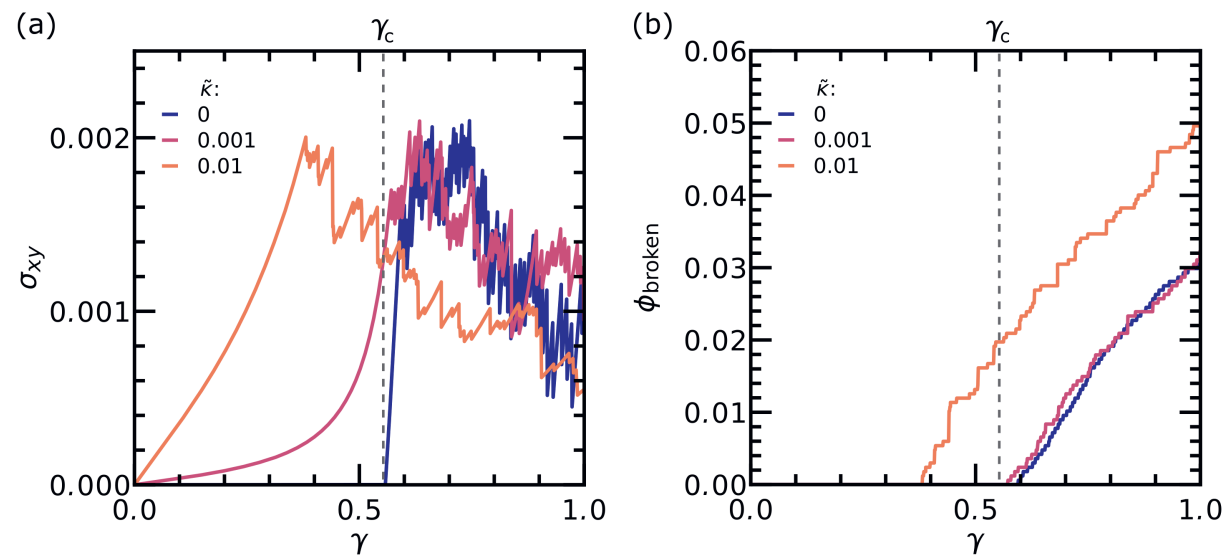

Figure 7.4. Bond scission prior to the critical strain at high bending rigidities and $\lambda=0.03$. Simulations are performed on diluted and distorted (three-dimensional) diamond lattices $(\langle z\rangle=3.4$, $L=5, N_{\text {bonds }} \approx 1650$ ). Bending interactions are considered between all nearest neighbour bonds. The FIRE-algorithm is used for energy minimization as described in Chapter 4 (Section 4.2.2). (a) Shear stress as a function of shear strain. (b) Fraction of broken bonds with respect to the initial number of bonds as a function of strain.

II in Figure 7.1). To what extent the initially floppy networks are stabilized can be expressed with a dimensionless number comparing the energy penalty for resisting the stabilizing field with the energy penalty for stretching. In case of bending this is the reduced bending rigidity $\tilde{\kappa}=\kappa /\left(\mu l_{0}^{2}\right)$, in the case of thermal fluctuations it is the reduced temperature $T^{*}=k_{\mathrm{B}} T /\left(\frac{\mu}{l_{0}} l_{0}^{2}\right)$, and for an embedding soft matrix it is $\tilde{\mu}_{2}=\frac{\mu_{2}}{l_{0}} l_{0}^{2} /\left(\frac{\mu}{l_{0}} l_{0}^{2}\right)=\mu_{2} / \mu$. The closer these values are to unity, the stronger the stabilizing fields.

\section{The fracture response of soft ductile polymer materials is primarily controlled by enthalpic chain stretching.}

By now, it is well accepted for athermal fibre networks, like collagen, that strainstiffening is caused by a rigidity transition from a bending-stabilized regime to a stretching-dominated regime. In contrast, the role of rigidity in the strain-stiffening behaviour of (semi-)flexible polymer networks is barely explored. The reason is that the global strain-stiffening of polymer networks is attributed to the entropic strain-stiffening behaviour of individual polymers, assuming that rearrangements at the network level are negligible. Indeed, we agree with literature that the initial strain-stiffening response is dominated by entropic strain-stiffening of individual chains. However, if the atomistic bonds are strong enough, individual polymer 
chains can be stretched significantly beyond their contour length into an elastic regime dominated by enthalpic stretching of the molecular bonds in the polymer chain ${ }^{13}$. Therefore, it is theoretically possible that in a polymer network a rigidity transition occurs from a regime stabilized by (non-linear) entropic chain stretching to a regime dominated by enthalpic chain stretching. Following the earlier findings presented in this thesis, this rigidity transition would result in large non-affine rearrangements and a strongly heterogeneous distribution in stress at the network level. The comparison of our simulation results for coarse-grained polymer networks with affine predictions in Chapter 6 (Figure 6.8) also points in this direction.

From an experimental point of view there has been little exploration in the role of enthalpic chain elasticity in the fracture response of polymer networks. Although this point of view has been proposed in literature ${ }^{36}$ and also starts to collect interest from the perspective of constitutive micromechanical modeling ${ }^{37}$, it deserves considerably more attention, because it has major implications for our understanding of the microscopic fracture process in polymer networks. It is possible that for soft brittle networks this regime dominated by enthalpic elasticity is not observed at the global level, because the enthalpic stretching regime is only reached around the defects prior to catastrophic fracture. However, based on our simulations in Chapter 6, we expect that soft ductile material remain intact beyond this rigidity transition. As a result, the majority of the fracture response of soft ductile materials should be dominated by enthalpic chain stretching. We note that in the coarse-grained network models, stabilizing fields typically lead to damping of non-affine fluctuations, in both the linear and non-linear regime ${ }^{38}$. This might provide a microscopic explanation for why the affine assumption still seems to work quite well for the elastic response of flexible polymer networks.

\section{Strong stabilizing fields decrease network extensibility.}

Up to this point we have assumed that during the fracture response the contribution of the stabilizing field is negligible. Generally this is true when the elements are very stiff compared to the stabilizing field. However, when the dimensionless numbers introduced in the previous paragraph get closer to unity, the stabilizing field can affect the stress distribution in the fracture regime and even lead to significant stretching prior to the athermal critical strain. Whether the stabilizing field can affect the fracture response, depends on the fracture strength of the elements in the network, which scales with $\lambda$ if the element is linearly elastic. With this information we introduce a new dimensionless number that measures the strength of the stabilizing field compared to the energy for rupture $E_{\text {break }}$; 
assuming that $E_{\text {break }}=\frac{1}{2} \frac{\mu}{l_{0}}\left(\lambda l_{0}\right)^{2}$, we can just divide the dimensionless numbers by $\frac{1}{2} \lambda^{2}$. In Figure 7.4 we show the fracture response of a strongly bending-stabilized network with $\lambda=0.03$. At $\tilde{\kappa}=1 \times 10^{-2}$ or $\tilde{\kappa}_{\text {break }}=\tilde{\kappa} /\left(\frac{1}{2} \lambda^{2}\right) \approx 20$ bonds start to break significantly before the central-force critical strain. Similarily, the effect of thermal fluctuations can be estimated by making a crude estimation of $T^{*}$ and $T_{\text {break }}^{*}$ in polymer networks based on single molecule experiments, picking $l_{0}=b$ (the Kuhn length). For a polyethylene glycol chain with $b=0.6 \mathrm{~nm}$ and $\frac{\mu}{l_{0}}=k_{\text {seg }}=20 \mathrm{nN} \mathrm{nm}^{-1}$, this would lead to $T^{*} \approx 1 \times 10^{-3}$ at $298 \mathrm{~K}^{2}$ and $T_{\text {break }}^{*}=1 \times 10^{-2} \cdot{ }^{13}$ At first glance, these values imply that enthalpic stretching dominates the fracture response of polymer networks as described above, however, this approach does not take into account the non-linear entropic response. A force balance as described for the extensible freely jointed chain in Chapter 6 (Section 6.2.4) could provide additional insight. To explore the use of $T^{*}$ and $T_{\text {break }}^{*}$, simulations could be considered with the network structures presented in Chapter 6, but now for phantom chains with harmonic bonds, such that the fracture response in presence of thermal fluctuations can be compared with the athermal fracture response. The observation that bonds can break prior to the athermal critical strain due to the stabilizing field might also be relevant beyond the accumulation of damage. In athermal central-force networks, crack nucleation is only possible after the critical strain. However, in the presence of a stabilizing field, enough stress might be able to concentrate around a large defect of size $c$ in a soft brittle network $\left(\sigma_{p} \sim \sqrt{1 / c}\right)$ to nucleate a crack, even if the bulk of the network is still below the critical strain. This phenomenon could explain why the enthalpic regime is barely observed for soft brittle networks.

\subsubsection{Additional fracture mechanisms in fibre networks}

So far we have only investigated brittle fracture by stretching as a bond scission mechanism. For molecular bonds this is indeed the most probable mode of fracture. However, in many biopolymer networks and supramolecular networks more fracture modes are possible due to their hierarchical nature.

\section{Ductile or plastic bond failure}

In Chapter 2 (Figure 2.11) we observed that for several collagen types, the peak strain $\gamma_{p}$ was significantly higher than the prediction from the simulations on our central-force elastic networks. In Ref. [39] we have attributed these higher values to plastic deformation either at the fibril level or at the network level depending on the type of collagen. Our hypothesis for fibril level plasticity is that the protofibrils that make up the collagen fibrils can slide along each other (Figure 7.5(a)), leading 
(a)

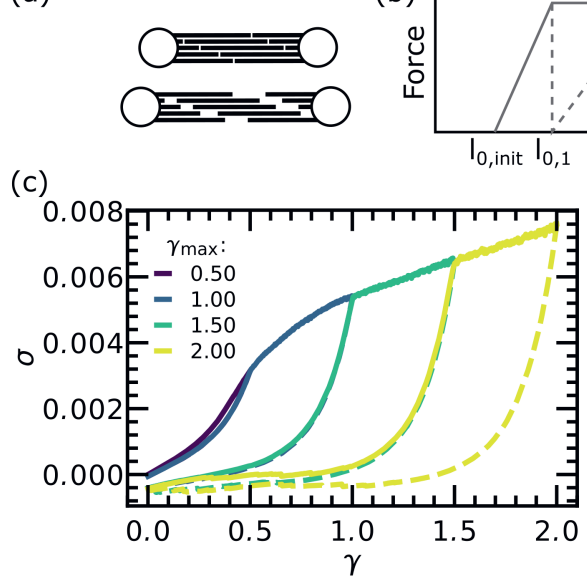

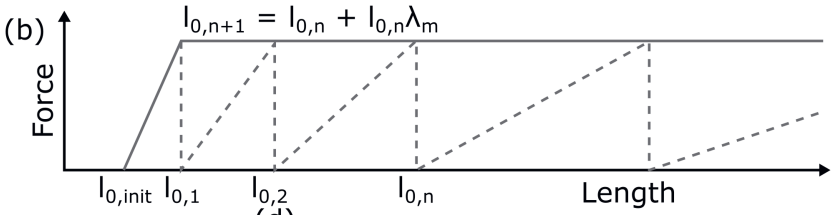

(d)

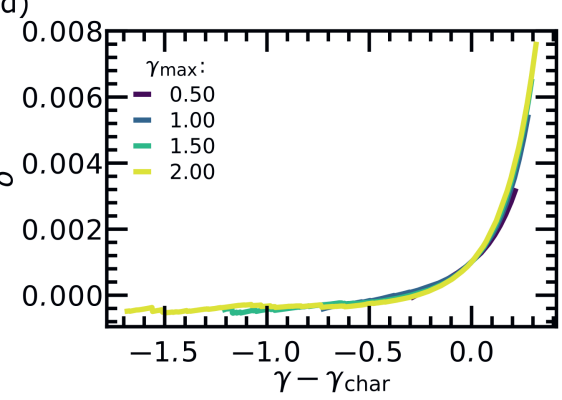

Figure 7.5. Damage accumulation via plastic bond failure. (a) Schematic of fibre plasticity by sliding. (b) Quasistatic description of the fibre lengthening process. In this example bond rupture is not considered. (c) Simulation results for repeated strain cycles performed on diluted and distorted (three-dimensional) diamond lattices where fibre plasticity is taken into account ( $\langle z\rangle=3.4, \tilde{\kappa}=1 \times 10^{-2}, \lambda_{m}=0.03, L=5, N_{\text {bonds }} \approx 1650$ ). We show forward (solid lines) and backward curves (dashed lines). The colour indicates the maximum strain $\gamma_{\text {max }}$ reached for that particular cycle (see legend). (d) Collapse of the backward curves in panel (c) based on the characteristic strain $\gamma_{\text {char }}$, defined as the strain at which the stress $\sigma_{\text {char }}=0.001$.

to lengthening of the fibrils, a phenomenon that has been demonstrated previously for fibrin and collagen networks ${ }^{40}$. In native collagen, the protofibrils inside the fibril are crosslinked to each other via telopeptides, which are attached at the ends of the protofibrils. Increased values of the peak strain $\gamma_{p}$ were observed for atelocollagen (see Figure 2.10), where these telopeptides are removed, indicating that the telopeptides prevent sliding of protofibrils. Alternatively, network plasticity is expected for telocollagen networks with a low connectivity $(\langle z\rangle=3)$. Those networks mainly consist out of branching collagen fibrils. Plasticity at the network level could occur by opening of these branches. We have introduced plasticity in our coarse-grained simulations as well, by introducing a single lengthening step prior to fracture at the plasticity threshold $\lambda_{m}$. The simulations show good agreement with the atelocollagen response and allow an estimation of the onset of fibre level plasticity. These results and the observations in Chapter 2 (Section 2.4) reveal that the fracture response of disordered fibre networks, can be tuned via network connectivity and the level of plastic deformation at the fibre level. 
We have developed a similar model to study the plastic response of synthetic tubule bundle networks that self-assemble from azo-benzene derived building blocks in collaboration with Groningen university ${ }^{41}$. This synthetic tubule bundle network has a wide plastic regime. In an effort to model this behaviour we have implemented a multi-step plastic response at the bond level as depicted schematically in Figure 7.5(b). By performing repeated strain cycles on our coarsegrained networks we can follow the evolution of the elastic response in the plastic regime (Figure 7.5(c)). Similar to repeated strain cycles on fibrin and collagen ${ }^{40}$ and the synthetic tubule bundle networks, the evolution in the elastic response of our simulation results can be collapsed on a master curve by shifting the curves with a characteristic strain $\gamma_{\text {char }}$, which is the strain at which the stress reaches a pre-defined threshold value $\sigma_{\text {char }}$ (Figure 7.5(d)). This collapse by subtraction indicates that lengthening does shift the onset of strain-stiffening, but does not affect the strain-stiffening process itself. We note that this plastic behaviour in fibril or tubule bundle networks bears resemblance to the plastic response of colloidal gels, which can be regarded as a network of colloidal gel strands, as demonstrated previously in our lab ${ }^{42,43}$. Experiments and simulations showed that the plastic response of these colloidal gels can also be attributed to yielding of the colloidal gel strands, which effectively lengthens the strands ${ }^{42,43}$. Considering this apparent overlap in the structure and mechanical response it seems worth exploring whether colloidal gels and (biological) bundle networks can be interpreted as limits of a design space for low-density hierarchical network materials.

\section{Breaking by peeling or snapping}

Another example of fibre networks with fracture properties that deviate from our central-force elastic model is the class of aromatic-dipeptide hydrogels. These dipeptides, functionalized at the N-terminus with an aromatic-group, self-assemble into fibre networks upon protonation of the C-terminus of the peptide sequence. The self-assembly is driven by $\pi-\pi$ interactions between Fmoc-dipeptides, and results in different fibrillar morphologies depending on the peptide sequence ${ }^{46}$.

In our lab we have studied the fracture response of Fmoc-Leu-Gly (Fmoc-LG), with Fmoc as the aromatic group and Leu-Gly the dipeptide, to challenge the applicability of our minimal network model to experimental systems ${ }^{45}$. FmocLG forms networks of stiff and long bundled ribbon-like fibres as shown in Figure 7.6(a $)^{44}$. Similar to biological fibre networks, Fmoc-LG networks show a non-linear response upon shear deformation and the development of a negative normal stress, which is characteristic of biopolymer gels (Figure 7.6). However, compared to collagen, the resulting fibre network is significantly stiffer and also 

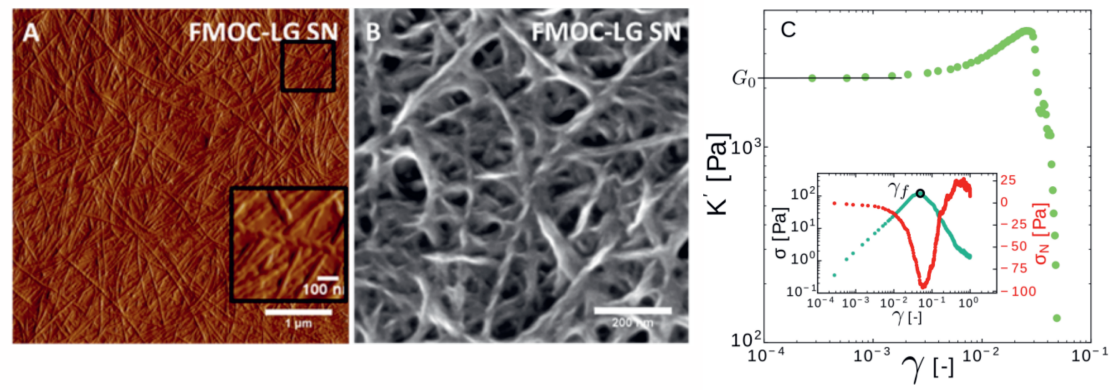

Figure 7.6. (a) AFM image of dried Fmoc-LG networks. (b) SEM image of dried Fmoc-LG network. Reprinted with permission from Ref. [44]. Copyright 2014 American Chemical Society. (c) Non-linear modulus $K^{\prime}$ as a function of the shear strain $\gamma$ for an FMOC-LG hydrogel. Inset shows both the shear stress and the normal stress as a function of $\gamma$. Plot based on data from Ref. [45].

the peak strain $\gamma_{p}$ is considerably lower $\left(\gamma_{p} \approx 0.05\right)$. As for most fibre networks, we expect that the Fmoc-LG fibre networks are subisostatic, which for a threedimensional structure most likely leads to a central-force critical strain above 0.05; hence, these Fmoc-LG networks seem to fracture prior to their critical strain. To confirm this hypothesis the network structure should be characterized in its hydrated state; however, to our knowledge this has not been achieved so far. Judging from atomic force microscopy and electron microscopy measurements on dried samples ${ }^{44}$ (Fig. 7.6(a) and (b)) we can estimate that the fibres have a radius of approximately $10 \mathrm{~nm}$ and a mesh size on the order of $100 \mathrm{~nm}$, so that for a solid rod the estimated $\tilde{\kappa}=\frac{1}{4}\left(r_{\text {fib }} / l_{c}\right)^{2} \approx 3 \times 10^{-3}$. This indicates that the stabilizing bending field in Fmoc-LG is strong compared to collagen ${ }^{47}$, but based on fracture simulation on our coarse-grained networks (Figure 2.3) we can not reproduce $\gamma_{p} \approx 0.05$ for this value of $\kappa$ with realistic $\lambda$ values (i.e. $\lambda \geq 0.5 \%$ ), even if we consider bending interactions between all neighbouring bonds to simulate a higher rigidity at the nodes (in our collagen models bending was only implemented along straight fibres). Simulations on random geometric graphs, which have a larger distribution in bond length, did not result in sufficiently low values for $\gamma_{p}$ either ${ }^{45,48}$. Peak strains of this order of magnitude have been observed in simulations of random fibre networks ${ }^{49}$ designed to model fracture of stiff fibre networks, like paper, where bonds between fibres can break both due to stretching and due to rotation induced peeling (see Figure 1.9(e)), showing that for stiff fibre networks alternative mechanisms of inter-fibre bond breaking can occur. Other mechanism that could be explored in the fracture of these stiff fibre networks are snapping of fibres due to bending and the effect of extremely long fibres, which 
have shown to supress non-affine deformation in the linear and non-linear elastic regime $^{50}$.

Aromatic-dipeptides seem to be a promising candidate for making synthetic fibre networks, as the building block is simple and bio-compatible. Currently, most of these networks are barely strain-stiffening and very brittle. However by carefully tuning their structure it might be possible to create networks with a strain-stiffening behaviour like collagen and a higher extensibility, expanding the range of applications for aromatic-dipeptide hydrogels.

\subsubsection{Elasticity and fracture of composite networks}

The second part of this thesis concerns the elasticity and fracture of composite networks. This choice was motivated by four observations:

- The prevalence of composite networks in biology ${ }^{51}$.

- The high level of tunability providing ample design space, combined with a synergistic response compared to the individual networks.

- The abundance of experimental data.

- The transition from a brittle to a ductile fracture response, suggesting a change in the microscopic fracture response.

\section{Tunability from locked in internal stress.}

For the elastic regime the high tunability is well demonstrated by our research in Chapter 4. By combining a network material that is soft at small strains and stiff at high strains, with a material that does not strain-stiffen in the region of interest, the linear and the non-linear regimes can be tuned independently. We also showed that the critical strain of a network can be shifted by incorporating a polyelectrolyte matrix material that builds up compressive internal stresses upon crosslinking. The result is similar to applying a compressive strain prior to shear deformation 7,35 . This approach effectively allows one to tune the strain-stiffening response. We hypothesized that the build-up of pre-stress during the polymerization process is caused by a combination of entropic and repulsive forces, but this hypothesis deserves some scrutiny. It is also not clear if the internal stress can be tuned after the crosslinking process via external parameters, such as $\mathrm{pH}$, salt concentration or temperature ${ }^{52}$, which could introduce additional functionality. It would be interesting to explore if the same principle can be realized with other material types as well or whether repulsive extensional stresses could be created such that the critical strain is shifted to lower strains. 


\section{Synergy arises from (emerging) constraints.}

Even more exciting than the tunability of composite networks is their synergistic stress response, i.e. the stress response of the composite is higher than the sum of the mechanical properties of the individual networks. The synergistic response arises from the interactions between the two networks, which are obviously not present in the separate materials. A perfect example is the enhanced linear modulus in double networks $47,53,54$ described in Chapter 4 (Figure 4.A.6), where the matrix resists the non-affine deformations that the fibre networks want to undergo. A requirement for a synergistic response is that the contribution of both networks to the mechanical property of interest is of roughly the same order of magnitude. This is the reason that there is barely any mechanical enhancement in the linear modulus of the polymer double networks in Chapter 6 (Figure 6.A.4): the sacrificial network dominates the overall response. Also there needs to be a mismatch in how the networks want to respond to a global deformation. If the networks respond in exactly the same way, as in the linear response of the double networks in Chapter 5, there will be no mechanical enhancement. The most striking example in mechanical enhancement we have observed was the enhancement in the fracture regime of the coarse-grained polymer double networks in Chapter 6 (Section 6.8). Because the networks can only interact via topological constraints, the enhancement only emerges when the chains in both networks are under tension. In this case, we found that the sacrificial chains contribute significantly to the mechanical response of the composite materials even at strains that are significantly higher than the fracture strain of a single sacrificial network.

\section{Locating the brittle-to-ductile transition.}

The most drastic outcome of constructing a composite network is that combining two brittle materials can result in a composite that behaves as a ductile material. Experiments show that there is great control over the transition from soft brittle to soft ductile via experimentally accessible parameters. We have explored this phenomenon both in Chapter 5 and Chapter 6. In Chapter 5 (Figure 5.5) we showed that for a highly coarse-grained model the location of the brittle-to-ductile transition can be predicted based on a force balance between the fracture strength of the matrix network and the reinforced sacrificial bond in the double network. It would be very helpful if the brittle-to-ductile transitions of real soft network materials could also be predicted based on this principle, as currently many experiments are required to identify the transition. Experiments do indicate that a force-balance also governs the brittle-to-ductile transition in experiment ${ }^{55}$. At a macroscopic level it means that the brittle-to-ductile transition should occur 
when the softer material in the neck can reach a higher stress than the yield stress. From Chapter 6 (Figure 6.3) we know that in the ductile regime the yield stress is defined solely by the sacrificial network and scales with the areal strand density. The fracture strength of the soft region, i.e. the global fracture strength of the double network, is a tougher nut to crack. Experiments suggest that the controlling parameter is the elastically effective strand density in the matrix, i.e. the density of polymer strands that contribute to the elastic response. Because the effective strand density $v_{\text {eff }}$ is corrected for topological constraints within and between networks, it can be significantly higher than just the chain density of the matrix network $v \cdot{ }^{55}$ Understanding the evolution in the density of topological constraints contributing to the elastic response might be a first step in locating the brittle-to-ductile transition more effectively. Assuming that in the fracture regime enthalpically stretched chains dominate the fracture response like in Chapter 6, this density could be determined in simulation by comparing the end-to-end distance between crosslinkers to the stretched contour length. If $L_{\text {stretched }} /(\mathrm{Nb})>1.0$ and $r_{\text {end-to-end }} /(\mathrm{Nb})<1.0$ at least one topological constraint will be present along the chain.

\subsection{Fracture and time}

The fracture process of soft network materials does not only involve several length scales, but also a range of time scales is relevant to the fracture process. In Chapter 3 (Section 3.A.4) we addressed three time related processes: thermal fluctuations, the rate of deformation and viscous damping effects. The influence of these effects is well understood for single bonds ${ }^{56,57}$; however, if and how these processes affect the microscopic fracture behaviour in networks remains unclear in most cases. The approach in Chapter 3 could be continued to identify these effects for fracture of systems that are driven externally. Below we will discuss a fracture process that we have not explored so far in this thesis: delayed fracture.

\subsubsection{Delayed fracture}

In delayed fracture a material breaks after a certain time $t_{\text {rupt }}$ even though it is loaded at a stress below the maximum strength ${ }^{58}$. From an engineering point of view, this type of fracture is undesirable due to its high unpredictability. From a theoretical point of view, the sudden speed-up of the fracture process as it passes from damage nucleation to damage propagation is fascinating. Two questions are typically asked. How far can $t_{\text {rupt }}$ be delayed and can catastrophic failure be forecasted? At a microscopic level, thermal fluctuations in the material are thought 
(a)

$$
\begin{gathered}
\downarrow \mathrm{k}_{\text {off }} \\
\mathrm{k}_{\text {off }}=\mathrm{k}_{\mathrm{off}, 0} \exp \left(\mathrm{f}_{\mathrm{i}}\right)
\end{gathered}
$$
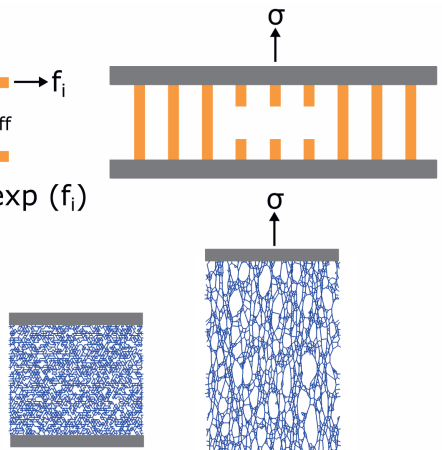

(c)

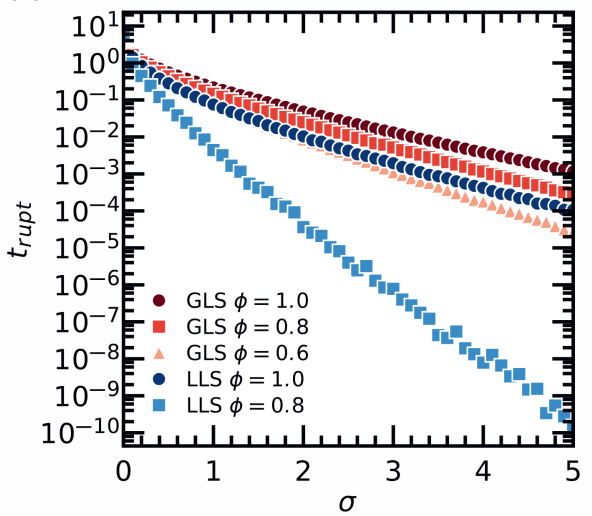

(b)

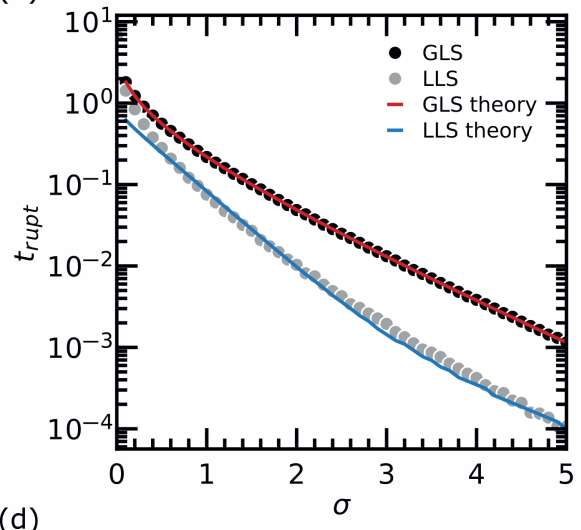

(d)

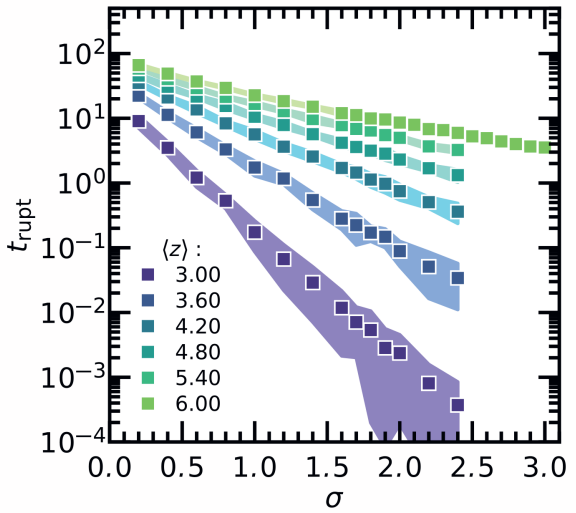

Figure 7.7. Studying delayed failure using the Gillespie model. (a) In the Gillespie model a force dependent rate constant $k_{\text {off }}$ is assigned to each bond. Which bond will break and the associated time step is determined based on these rate constants. We have implemented this procedure for a fibre bundle model (top), where the redistribution of load is predefined according to a load sharing rule and in a network model (bottom) where the redistribution of load after bond rupture is determined by the energy minimization procedure introduced in Chapter 2. To achieve a constant stress in the latter model the strain is adjusted iteratively between Gillespie steps until the desired stress is measured. Note that bonds are only broken during Gillespie steps. (b) Rupture time $t_{\text {rupt }}$ versus applied stress $\sigma$ for the bundle model global load sharing (GLS) and local load sharing (LLS) rules. Dots represent simulations results, while the lines are the theoretical predictions. (c) Influence of defects on $t_{\text {rupt }}$ as a function of $\sigma . \phi$ indicates the fraction of remaining bonds after random deletion. (d) $t_{\text {rupt }}$ as a function of $\sigma$ for a central-force spring model with average connectivity $\langle z\rangle$ coupled to a Gillespie model. The stress is kept constant by adjusting the strain accordingly. 
to be responsible for delayed fracture. Due to their stochastic nature there is a very small possibility that a thermal fluctuation occurs that is large enough to induce bond scission. Thus, even if a material is not being deformed this process will slowly lead to the degradation of a material, provided that bond scission is irreversible. If instead the material is experiencing a subcritical load, this process will speed up significantly. Since the bonds in the materials will be pre-stressed, the probability that a thermal fluctuations occurs that is large enough to break the bond is significantly higher ${ }^{56}$. Once a defect is created from this initially random process, stress will concentrate and the fracture process speeds up drastically.

To explore the tunability of delayed fracture we studied the effect of load redistribution on delayed fracture in a one-dimensional fibre bundle model ${ }^{59}$ similar to Ref. [60] as shown in Figure 7.7. To capture the stochastic nature of fracture in the presence of thermal fluctuations, rupture and rupture time of the fibres is determined by a Gillespie algorithm ${ }^{61}$. In the simplest model load is shared equally over all fibres (global load sharing or GLS), so that after rupture the load is redistributed equally over all remaining fibres. Even though bond rupture is stochastic, the average rupture time of this model can easily be predicted in the limit of global load sharing, because stress cannot concentrate (Figure 7.7). The other limit with respect to stress redistribution is the local load sharing (LLS) limit, where after bond rupture load is only distributed over the neighbouring fibres. Predicting the rupture time is more involved in this model, because the stress concentration at the crack edges significantly accelerates once the crack reaches a critical crack size $l^{*}$. Nevertheless we were able to describe the rupture time, using only one fitting parameter, by assuming that $t_{\text {rupt }}$ is only determined by the time required to generate a crack of size $l^{*}$ and that the process of damage accumulation is random up to this point ${ }^{59}$. The fibre bundle model illustrates perfectly that the method of load redistribution controls the sensitivity to defects (Figure 7.7(c)). Thus in studying delayed fracture it is important to know how load is shared in a system.

In a network, load sharing is not specified, but emergent from the network structure. To study effect of network structure on delayed fracture, we coupled the energy minimization procedure for central-force networks, described in Chapter 2 (Section 2.3), to the Gillespie algorithm (see Figure 7.7 for initial results). This Gillespie central-force network model allows us to study the effect of network structure on delayed fracture and in particular the microscopic mechanisms that precede catastrophic fracture for systems where structural relaxation effects can be neglected. One intriguing possibility is to study the relation between the inhomogeneous stress distribution arising in networks and damage reporting by mechanophores as described theoretically for pre-defined load sharing in Ref. [62]. 


\subsubsection{Rupture kinetics and reversibility}

The bonds described in the previous section are also called slip bonds. A hottopic in mechanobiology, especially in cell adhesion ${ }^{63}$, is a mechanical bond with different kinetics: the catch bond. In contrast to slip bonds, the life-time of a catch bond initially increases if the bond is stressed, induced by conformation changes at the molecular level (Figure 7.8(a)). By using a constitutive description of catch bond kinetics, we can explore the role of catch bonding in network fracture with our Gillespie central-force network model as demonstrated in Figure 7.8(b). We show that the bond kinetics does translate to the response of the network, however, we expect that the true power of catch bonding comes into play when the catch bond formation is reversible and the catch bonds are mobile (Figure 7.8(c)), so that catch bonds can accumulate at locations of intermediate stress, prolonging the lifetime ${ }^{64}$ and building in a short term structural memory with respect to applied load. So far we have introduced reversibility with pre-existing bonds (Figure 7.8(d)), which introduces a meta-stable regime which has also been observed for FBMs in Ref. [64]. Future studies can employ this type of model to study the dynamics of (micro)cracks in transient and self-healing materials.

\subsection{Bridging length scales}

In this thesis we have focused on fracture processes at the mesoscopic level. However, there is a vast amount of literature on the study of fracture from a continuum point of view ${ }^{65-67}$ as discussed in Section 1.3. Will it be possible to bridge the gap between a mesoscopic understanding of fracture, as discussed in this thesis, and the continuum approach?

The continuum elasticity point of view always considers the propagation of cracks resulting from stress concentration at the crack tip of a pre-existing defect in the material. The material surrounding this defect is considered a continuum and a clear interface exists between the defect and the bulk of the material. In contrast, a network could be interpreted as a material only consisting of voids, the empty spaces bounded by bonds. It is not immediately clear if and how a defect can be distinguished from these voids. The most probable candidates are rare large-sized voids that emerge from network disorder or large voids that are created by merging of smaller voids during diffuse failure (a process that lies out of the scope of continuum elasticity). However, whether stress can concentrate at the edges of these voids will not only depend on their size, but also on the structure of the surrounding network. To explore this problem further we recommend a study 
(a)

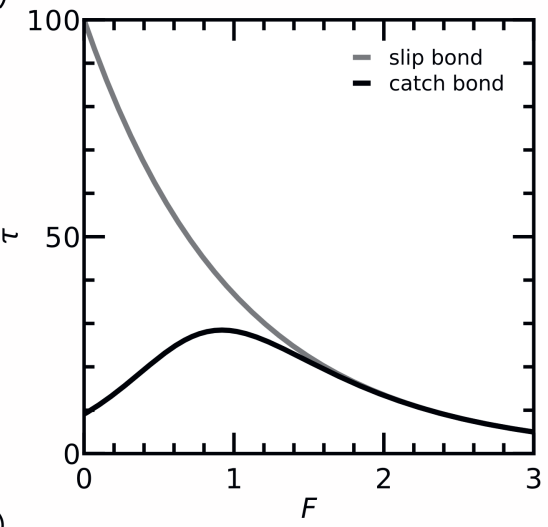

(c)

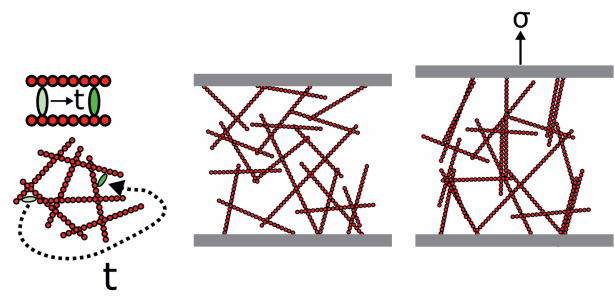

(d) (b)

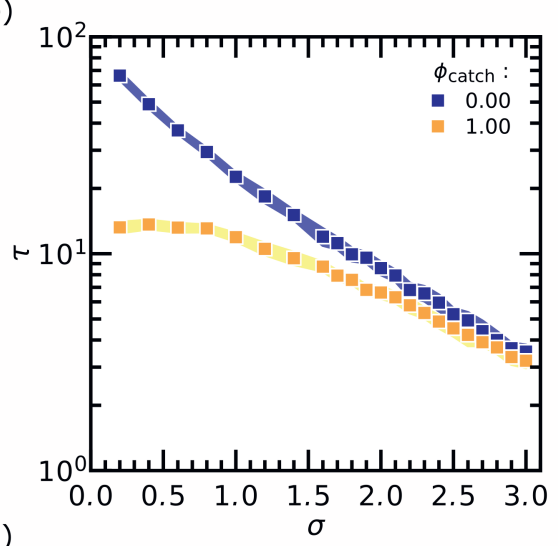

(e)

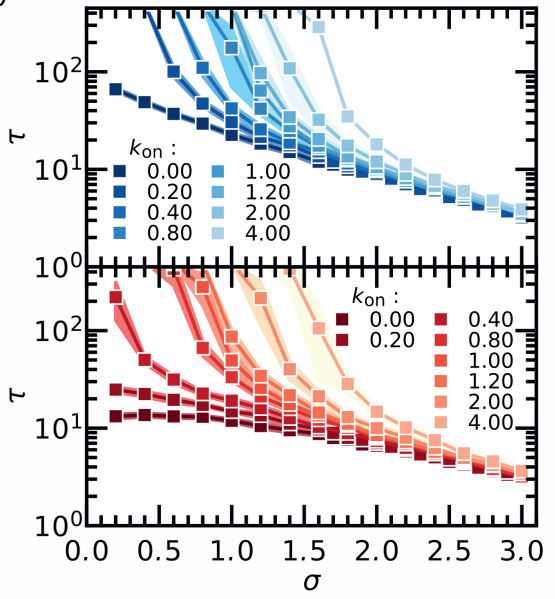

Figure 7.8. Rupture kinetics and reversibility. (a) Force $F$ versus life-time $\tau=1 / k_{\text {off }}$ curves for slip bonds and catch bonds used in this model. (b) $t_{\text {rupt }}$ as a function of $\sigma$ for Gillespie-central-force spring network models with $\langle z\rangle=6.0$. $\phi_{\text {catch }}$ is the fraction of catch bonds in the network. (c) Two types of mobility, translation along fibres and diffusion, in combination with reversible binding allows network reorganization in a response to stress. (d) Introduction of reversible binding of pre-existing bonds. (e) $t_{\text {rupt }}$ as a function of $\sigma$ for networks with $\langle z\rangle=6.0$ for a range of $k_{\text {on }}$. The networks are not equilibrated prior to deformation. We observe a shift to a metastable state at low stress with increasing $k_{\text {on }}$ for slip bonds (top) and catch bonds (bottom). 
on stress profiles around cracks of size $c$ as a function of the network connectivity in the central-force elastic model.

At which stress a large defect will propagate is largely determined by the fracture energy $\Gamma$. From a continuum elasticity point of view the fracture energy is the sum of the intrinsic fracture energy $\Gamma_{0}$ required to break the bonds that span the future crack and the dissipative fracture energy $\Gamma_{D}$ that captures the dissipation processes around this crack in order to allow crack propagation. In real materials both these parameters can be controlled by a range of physical phenomena such as network heterogeneity by pre-stretch in polymer networks, molecular friction in elastomers and sharp gradients in the chemical potential at the crack-tip in biological hydrogels ${ }^{68}$. However, for our central-force elastic model it might be possible to systematically explore both $\Gamma_{0}$ and $\Gamma_{D}$ as a function of network structure, allowing the possibility to provide a microscopic explanation of the fracture energy $\Gamma$ that goes beyond the Lake-Thomas approach ${ }^{69}$. Experiments on the well-defined tetra-PEG gels might be a good starting point in this respect, as their fracture toughness is close to $\Gamma_{0}{ }^{70}$. In particular the study of the stress and damage profiles surrounding artificially introduced cracks could be helpful. For example, for random fuse networks it has been demonstrated that a cross-over occurs from disorder dominated behaviour to stress concentration dominated behaviour as a function of the crack length ${ }^{71}$. Recently this transition has also been demonstrated in tensile experiments ${ }^{72,73}$ and puncture experiments ${ }^{74}$ on polyacrylamide gels and cavitation experiments in PDMS ${ }^{66}$. As explained in Section $1.3, \Gamma$ is connected to the non-linear length scale $\ell$ and a dissipative length scale $\xi$ in the continuum description. Identifying $c$ dependent cross-overs in the fracture response of the central-force isostatic model, could provide a mesoscopic interpretation of these length scales. These results could then be used to design materials with a tunable response from brittle to ductile solely based on structure, either by modifying $c$ or by tuning $\xi$ and $\ell$.

\subsection{Conclusion and outlook}

In this thesis we have explored how soft network materials deal with stress at the mesoscopic level both during their elastic and fracture response. We have demonstrated that the combination of large deformability and network level disorder in soft materials leads to fascinating mechanics, both in the reversibly elastic regime, where a force balance between enthalpic stretching and a stabilizing field controls the mechanical response, and in the fracture regime, where stress heterogeneity induced by network rigidity controls the fracture response. 
Significant steps are still required to achieve a microscopic understanding of fracture in our minimal central-force elastic network model as outlined in the general discussion, in particular for the subisostatic regime. The biggest challenge will be to establish a strong connection between this model and the evolution in the fracture response from diffuse damage to crack propagation in real soft network materials. Especially the transition from diffuse fracture to crack nucleation deserves attention, as these stages occur in every fracture response, but are so far poorly explored in soft network materials. From the perspective of simulations, this challenge initially requires a more systematic analysis of the microscopic fracture response in the current central-force elastic model, rather than the development of new simulation methods. A follow up step would be to study fracture in coarsegrained polymer networks both with and without excluded volume interactions between chains ${ }^{75}$ such that the effect of topological constraints on fracture can be explored systematically. On the experimental side, mechanophores can play a major role in increasing the understanding of damage accumulation in this stage of the fracture response, for example by reporting on spatial and temporal correlation in the damage process in polymer networks with existing mechanophores ${ }^{76}$, but also by developing mechanophores that can provide insight in the evolution of the chain-stretch distribution. On top of that, biological or synthetic fibre networks could play an important role as the coarseness of their networks in principle allows us to track damage accumulation at the network level in space and time. Typically fluorophores are used to allow visualization via confocal laser scanning microscopy, but considering the development in mechanophores it might also be possible to incorporate mechanophores into the fibres such that they can provide insight into stress heterogeneity in the fibre network, e.g. by crosslinking the protofibrils in the collagen strands.

The research described in this thesis has followed the classical philosophy regarding fracture, that failure is an inevitable process. For those materials we have now shown that the damage process can be controlled via network structure or strong stabilizing fields. Especially, the creation of double networks has proven to be an effective strategy to create deformable, tough, and strong networks. Both for these polymer double networks and our work on biological composites the incorporation of internal stresses, is an effective way to alter the mechanical response. In creating tough networks the transition from soft brittle to soft ductile materials plays a crucial role. However, also for self-healing materials a ductile fracture response, with a large diffuse failure regime, might make the material more resilient as local damage must be easier to repair than large cracks.

Shifting our focus to networks in biology, we expect that the fracture response of biological soft networks deviates from network materials used in technological 
applications, in several aspects. This is related to both the softness of these networks and their coarseness. For most biological fibre networks $L / l_{c}$ is very small, not only because $l_{c}$ is relatively high, but also because the typical network size $L$ in biological networks, such as the cytoskeleton or the collagen network in the wall of the aorta ${ }^{77}$, is small. Therefore, a continuum picture seems less relevant in the biological context and a shift to more damage percolation like fracture is expected (Figure 7.3). A question that arises is whether biological systems can or even do exploit this size effect. Although networks with smaller $L / l_{c}$ will relatively be stronger and tougher, networks with large $l_{c}$ will also have a lower strand density reducing their absolute strength. Finally, interactions in biological systems can be highly dynamic, allowing a drastically different philosophy towards fracture: accumulation of damage is not problematic as long as it can be repaired. This dynamic character in combination with the inherent inhomogeneous stress distribution in networks could serve as a governing principle behind "smart" ways to deal with stress. 


\section{List of symbols}

\begin{tabular}{|c|c|}
\hline Symbol & Description \\
\hline$c$ & Size of a defect or (micro)crack \\
\hline$E_{\text {break }}$ & Energy required to rupture a bond \\
\hline$k_{\mathrm{B}}$ & Boltzmann constant \\
\hline$k_{\text {off }}$ & Load dependent unbinding rate of a bond in the Gillespie model \\
\hline$k_{\text {off }, 0}$ & Unbinding rate of a bond if no load is applied \\
\hline$k_{\text {on }}$ & Load independent rebinding rate of a bond in the Gillespie model \\
\hline$k_{\text {seg }}$ & Spring constant of a polymer chain segment \\
\hline$l_{0}$ & Segment rest length \\
\hline$l_{c}$ & Equilibrium distance between crosslinkers \\
\hline$l_{p}$ & Persistence length \\
\hline$l_{*}$ & Critical crack size for crack propagation in the Gillespie model \\
\hline$\ell$ & Non-linear length scale in fracture mechanics \\
\hline$L$ & System size \\
\hline$L_{\text {contour }}$ & Contour length of a (semiflexible) polymer chain \\
\hline$L_{\text {stretched }}$ & Contour length of a polymer chain in its stretched state \\
\hline$L^{*}$ & Characteristic length scale related to the fracture response \\
\hline$N_{\text {bonds }}$ & Number of bonds in the network \\
\hline$p$ & Connectivity parameter for (diluted) triangular lattices \\
\hline$p_{c}$ & The isostatic point \\
\hline$r, r_{1 \mathrm{st}}, r_{2 \mathrm{nd}}$ & Rigidity, first order rigidity, and second order rigidity \\
\hline$r_{\text {fib }}$ & Fibre radius \\
\hline$r_{\text {end-to-end }}$ & End-to-end distance of a polymer chain \\
\hline$t_{\text {rupt }}$ & Time until rupture for a material under a subcritical load \\
\hline$T$ & Temperature \\
\hline$T^{*}$ & Reduced temperature \\
\hline$T_{\text {break }}^{*}$ & $\begin{array}{l}\text { Dimensionless number expressing the sensitivity of bond rupture } \\
\text { to thermal fluctuations }\end{array}$ \\
\hline$\langle z\rangle$ & Average connectivity \\
\hline$\beta$ & Inverse disorder \\
\hline$\gamma$ & Shear strain \\
\hline$\gamma_{\text {char }}$ & Characteristic shear strain at which the stress equals $\sigma_{\text {char }}$ \\
\hline$\gamma_{\max }$ & Maximum shear strain experienced by the network \\
\hline
\end{tabular}


$\gamma_{p}$

$\Gamma$

$\Gamma_{0}$

$\Gamma_{D}$

$\epsilon_{C}$

$\epsilon_{p}$

$\kappa$

$\tilde{\kappa}$

$\tilde{\kappa}_{\text {break }}$

$\lambda$

$\lambda_{m}$

$\delta \lambda$

$\mu$

$\mu_{2}$

$\tilde{\mu}_{2}$

$v$

$v_{\text {eff }}$

$\xi$

$\sigma$

$\sigma_{p}$

$\sigma_{\text {char }}$

$\phi$

$\phi_{\text {catch }}$

Peak strain

Fracture energy

Intrinsic fracture energy

Dissipative fracture energy

Critical extensional strain

Peak strain or fracture strain

Bending rigidity

Reduced bending rigidity

Dimensionless number expressing the sensitivity of bond rupture to fibre bending

Rupture threshold

Lengthening threshold

Width of the distribution in the rupture threshold

Stretching rigidity

Stretching rigidity of a matrix

Reduced stretching rigidity of a matrix

Polymer chain or strand density.

Effective strand density, including topological constraints.

Dissipative length scale in fracture mechanics

Stress

Peak stress, fracture stress or strength

Stress threshold used in the rescaling of the mechanical response under plastic deformation

Fraction of remaining bonds in the 1D Gillespie model after random deletion

Fraction of catch bonds in the Gillespie model with respect to all intact bonds 


\section{References}

[1] S. Arzash, J. L. Shivers, F. C. MacKintosh, Finite size effects in critical fiber networks. Soft Matter 16, 6784-6793 (2020).

[2] L. Zhang, D. Z. Rocklin, L. M. Sander, X. Mao, Fiber networks below the isostatic point: Fracture without stress concentration. Physical Review Materials 1, 052602 (2017).

[3] A. Sharma, A. J. Licup, K. A. Jansen, R. Rens, M. Sheinman, G. H. Koenderink, F. C. Mackintosh, Strain-controlled criticality governs the nonlinear mechanics of fibre networks. Nature Physics 12, 584-587 (2016).

[4] M. Merkel, K. Baumgarten, B. P. Tighe, M. L. Manning, A minimal-length approach unifies rigidity in underconstrained materials. Proceedings of the National Academy of Sciences of the United States of America 116, 6560-6568 (2019).

[5] J. L. Shivers, S. Arzash, A. Sharma, F. C. MacKintosh, Scaling Theory for Mechanical Critical Behavior in Fiber Networks. Physical Review Letters 122, 188003 (2019).

[6] O. K. Damavandi, V. F. Hagh, C. D. Santangelo, M. L. Manning, Energetic Rigidity: a Unifying Theory of Mechanical Stability. Preprint at http://arxiv.org/abs/2102.11310 (2021).

[7] S. Arzash, J. L. Shivers, A. J. Licup, A. Sharma, F. C. Mackintosh, Stress-stabilized subisostatic fiber networks in a ropelike limit. Physical Review E 99, 042412 (2019).

[8] M. E. Cates, J. Wittmer, J.-P. P. Bouchaud, P. Claudin, Jamming, Force Chains, and Fragile Matter. Physical Review Letters 81, 1841-1844 (1998).

[9] J. H. Snoeijer, T. J. Vlugt, M. van Hecke, W. van Saarloos, Force Network Ensemble: A New Approach to Static Granular Matter. Physical Review Letters 92, 054302 (2004).

[10] S. Ostojic, E. Somfai, B. Nienhuis, Scale invariance and universality of force networks in static granular matter. Nature 439, 828-830 (2006).

[11] L. Papadopoulos, M. A. Porter, K. E. Daniels, D. S. Bassett, Network analysis of particles and grains. Journal of Complex Networks 6, 485-565 (2018).

[12] M. Guthold, W. Liu, E. A. Sparks, L. M. Jawerth, L. Peng, M. Falvo, R. Superfine, R. R. Hantgan, S. T. Lord, A comparison of the mechanical and structural properties of fibrin fibers with other protein fibers. Cell Biochemistry and Biophysics 49, 165-181 (2007).

[13] S. Wang, S. Panyukov, M. Rubinstein, S. L. Craig, Quantitative Adjustment to the Molecular Energy Parameter in the Lake-Thomas Theory of Polymer Fracture Energy. Macromolecules 52, 2772-2777 (2019).

[14] Y. Mao, B. Talamini, L. Anand, Rupture of polymers by chain scission. Extreme Mechanics Letters 13, 17-24 (2017).

[15] T. Matsuda, T. Nakajima, Y. Fukuda, W. Hong, T. Sakai, T. Kurokawa, U. I. Chung, J. P. Gong, Yielding Criteria of Double Network Hydrogels. Macromolecules 49, 1865-1872 (2016). 
[16] P. Millereau, E. Ducrot, J. M. Clough, M. E. Wiseman, H. R. Brown, R. P. Sijbesma, C. Creton, Mechanics of elastomeric molecular composites. Proceedings of the National Academy of Sciences of the United States of America 115, 9110-9115 (2018).

[17] M. Bacca, C. Creton, R. M. McMeeking, A Model for the Mullins Effect in Multinetwork Elastomers. Journal of Applied Mechanics 84, 121009 (2017).

[18] I. Malakhovsky, M. A. Michels, Scaling and localization in fracture of disordered central-force spring lattices: Comparison with random damage percolation. Physical Review B 74, 014206 (2006).

[19] E. Berthier, M. A. Porter, K. E. Daniels, Forecasting failure locations in 2-dimensional disordered lattices. Proceedings of the National Academy of Sciences of the United States of America 116, 16742-16749 (2019).

[20] D. M. Sussman, C. P. Goodrich, A. J. Liu, Spatial structure of states of self stress in jammed systems. Soft Matter 12, 3982-3990 (2016).

[21] E. Lerner, Quasilocalized states of self stress in packing-derived networks. European Physical Journal E 41, 93 (2018).

[22] M. F. Vermeulen, A. Bose, C. Storm, W. G. Ellenbroek, Geometry and the onset of rigidity in a disordered network. Physical Review E 96, 053003 (2017).

[23] A. Shekhawat, S. Zapperi, J. P. Sethna, From damage percolation to crack nucleation through finite size criticality. Physical Review Letters 110, 185505 (2013).

[24] M. J. Alava, P. K. V. V. Nukala, S. Zapperi, Statistical models of fracture. Advances in Physics 55, 349-476 (2006).

[25] D. Bonamy, E. Bouchaud, Failure of heterogeneous materials: A dynamic phase transition? Physics Reports 498, 1-44 (2011).

[26] M. M. Driscoll, B. G. G. Chen, T. H. Beuman, S. Ulrich, S. R. Nagel, V. Vitelli, The role of rigidity in controlling material failure. Proceedings of the National Academy of Sciences of the United States of America 113, 10813-10817 (2016).

[27] S. Roux, A. Hansen, H. Herrmann, E. Guyon, Rupture of heterogeneous media in the limit of infinite disorder. Journal of Statistical Physics 52, 237-244 (1988).

[28] C. Manzato, A. Shekhawat, P. K. V. V. Nukala, M. J. Alava, J. P. Sethna, S. Zapperi, Fracture strength of disordered media: Universality, interactions, and tail asymptotics. Physical Review Letters 108, 065504 (2012).

[29] M. Sahimi, S. Arbabi, Mechanics of disordered solids. III. Fracture properties. Physical Review B 47, 713-722 (1993).

[30] P. K. V. Nukala, S. Zapperi, S. Åimunović, Statistical properties of fracture in a random spring model. Physical Review E 71, 066106 (2005).

[31] A. Guarino, L. Vanel, R. Scorretti, S. Ciliberto, The cooperative effect of load and disorder in thermally activated rupture of a two-dimensional random fuse network. Journal of Statistical Mechanics: Theory and Experiment p. P06020 (2006). 
[32] J. Kierfeld, V. M. Vinokur, Slow crack propagation in heterogeneous materials. Physical Review Letters 96, 175502 (2006).

[33] G. H. Koenderink, Z. Dogic, F. Nakamura, P. M. Bendix, F. C. MacKintosh, J. H. Hartwig, T. P. Stossel, D. A. Weitz, An active biopolymer network controlled by molecular motors. Proceedings of the National Academy of Sciences of the United States of America 106, 15192-15197 (2009).

[34] C. P. Broedersz, F. C. MacKintosh, Molecular motors stiffen non-affine semiflexible polymer networks. Soft Matter 7, 3186 (2011).

[35] A. S. G. Van Oosten, M. Vahabi, A. J. Licup, A. Sharma, P. A. Galie, F. C. MacKintosh, P. A. Janmey, Uncoupling shear and uniaxial elastic moduli of semiflexible biopolymer networks: compression-softening and stretch-stiffening. Scientific Reports 6, 19270 (2016).

[36] D. E. Hanson, J. L. Barber, A new paradigm for the molecular basis of rubber elasticity. Contemporary Physics 56, 319-337 (2015).

[37] M. R. Buche, M. N. Silberstein, Chain breaking in the statistical mechanical constitutive theory of polymer networks. Preprint at https://arxiv.org/abs/2104.08866 (2021).

[38] A. R. Cioroianu, E. M. Spiesz, C. Storm, Disorder, pre-stress and non-affinity in polymer 8-chain models. Journal of the Mechanics and Physics of Solids 89, 110-125 (2016).

[39] F. Burla, S. Dussi, C. Martinez-Torres, J. Tauber, J. van der Gucht, G. H. Koenderink, Connectivity and plasticity determine collagen network fracture. Proceedings of the National Academy of Sciences of the United States of America 117, 8326-8334 (2020).

[40] S. Munster, L. M. Jawerth, B. a. Leslie, J. I. Weitz, B. Fabry, D. a. Weitz, Strain history dependence of the nonlinear stress response of fibrin and collagen networks. Proceedings of the National Academy of Sciences of the United States of America 110, 12197-12202 (2013).

[41] J. W. Fredy, A. Méndez-Ardoy, S. Kwangmettatam, D. Bochicchio, B. Matt, M. C. Stuart, J. Huskens, N. Katsonis, G. M. Pavan, T. Kudernac, Molecular photoswitches mediating the strain-driven disassembly of supramolecular tubules. Proceedings of the National Academy of Sciences of the United States of America 114, 11850-11855 (2017).

[42] J. M. Van Doorn, J. E. Verweij, J. Sprakel, J. Van Der Gucht, Strand Plasticity Governs Fatigue in Colloidal Gels. Physical Review Letters 120, 208005 (2018).

[43] J. E. Verweij, F. A. Leermakers, J. Sprakel, J. Van Der Gucht, Plasticity in colloidal gel strands. Soft Matter 15, 6447-6454 (2019).

[44] W. H. Rombouts, M. Giesbers, J. Van Lent, F. A. De Wolf, J. Van Der Gucht, Synergistic stiffening in double-fiber networks. Biomacromolecules 15, 1233-1239 (2014).

[45] P. de Visser, Disentangling the mechanical behavior of a synthetic fiber network, Master's thesis, Wageningen University and Research (2019).

[46] S. Fleming, R. V. Ulijn, Design of nanostructures based on aromatic peptide amphiphiles. Chemical Society Reviews 43, 8150-8177 (2014). 
[47] F. Burla, J. Tauber, S. Dussi, J. van der Gucht, G. H. Koenderink, Stress management in composite biopolymer networks. Nature Physics 15, 549-553 (2019).

[48] F. Beroz, D. Zhou, X. Mao, D. K. Lubensky, Physical limits to sensing material properties. Nature Communications 11, 5170 (2020).

[49] S. Deogekar, R. C. Picu, On the strength of random fiber networks. Journal of the Mechanics and Physics of Solids 116, 1-16 (2018).

[50] C. P. Broedersz, M. Sheinman, F. C. MacKintosh, Filament-length-controlled elasticity in 3D fiber networks. Physical Review Letters 108, 078102 (2012).

[51] F. Burla, Y. Mulla, B. E. Vos, A. Aufderhorst-Roberts, G. H. Koenderink, From mechanical resilience to active material properties in biopolymer networks. Nature Reviews Physics 1, 249-263 (2019).

[52] P. de Almeida, M. Jaspers, S. Vaessen, O. Tagit, G. Portale, A. E. Rowan, P. H. Kouwer, Cytoskeletal stiffening in synthetic hydrogels. Nature Communications 10, 609 (2019).

[53] A. S. Shahsavari, R. C. Picu, Exceptional stiffening in composite fiber networks. Physical Review E 92, 012401 (2015).

[54] J. M. Van Doorn, L. Lageschaar, J. Sprakel, J. Van Der Gucht, Criticality and mechanical enhancement in composite fiber networks. Physical Review E 95, 042503 (2017).

[55] S. Ahmed, T. Nakajima, T. Kurokawa, M. Anamul Haque, J. P. Gong, Brittle-ductile transition of double network hydrogels: Mechanical balance of two networks as the key factor. Polymer 55, 914-923 (2014).

[56] H. Kramers, Brownian motion in a field of force and the diffusion model of chemical reactions. Physica 7, 284-304 (1940).

[57] J. T. Bullerjahn, S. Sturm, K. Kroy, Theory of rapid force spectroscopy. Nature Communications 5, 4463 (2014).

[58] H. M. Van Der Kooij, S. Dussi, G. T. Van De Kerkhof, R. A. Frijns, J. Van Der Gucht, J. Sprakel, Laser Speckle Strain Imaging reveals the origin of delayed fracture in a soft solid. Science Advances 4, eaar1926 (2018).

[59] H. Dekker, Exploiting rigidity and surface structure to tune adhesive performance, Master's thesis, Wageningen University and Research (2019).

[60] Y. Mulla, G. Oliveri, J. T. Overvelde, G. H. Koenderink, Crack Initiation in Viscoelastic Materials. Physical Review Letters 120, 268002 (2018).

[61] D. T. Gillespie, A general method for numerically simulating the stochastic time evolution of coupled chemical reactions. Journal of Computational Physics 22, 403-434 (1976).

[62] F. F. Dubach, W. G. Ellenbroek, C. Storm, How accurately do mechanophores report on bond scission in soft polymer materials? Journal of Polymer Science 59, 1188-1199 (2021). 
[63] E. V. Sokurenko, V. Vogel, W. E. Thomas, Catch-Bond Mechanism of Force-Enhanced Adhesion: Counterintuitive, Elusive, but ... Widespread? Cell Host and Microbe 4, 314-323 (2008).

[64] Y. Mulla, M. J. Avellaneda, A. Roland, L. Baldauf, S. J. Tans, G. H. Koenderink, Weak catch bonds make strong networks. Preprint at http:/ / www.biorxiv.org/content/10.1101/2020.07.27.219618v2 (2020).

[65] C. Creton, M. Ciccotti, Fracture and adhesion of soft materials: A review. Reports on Progress in Physics 79, 46601 (2016).

[66] J. Y. Kim, Z. Liu, B. M. Weon, T. Cohen, C. Y. Hui, E. R. Dufresne, R. W. Style, Extreme cavity expansion in soft solids: Damage without fracture. Science Advances 6, eaaz0418 (2020).

[67] R. Long, C. Y. Hui, J. P. Gong, E. Bouchbinder, The Fracture of Highly Deformable Soft Materials: A Tale of Two Length Scales. Annual Review of Condensed Matter Physics 12, 71-94 (2021).

[68] K. Bircher, M. Zündel, M. Pensalfini, A. E. Ehret, E. Mazza, Tear resistance of soft collagenous tissues. Nature Communications 10, 792 (2019).

[69] G. Lake, A. Thomas, The strength of highly elastic materials. Proceedings of the Royal Society of London. Series A. Mathematical and Physical Sciences 300, 108-119 (1967).

[70] T. Sakai, Y. Akagi, S. Kondo, U. Chung, Experimental verification of fracture mechanism for polymer gels with controlled network structure. Soft Matter 10, 6658-6665 (2014).

[71] M. J. Alava, P. K. V. V. Nukala, S. Zapperi, Role of disorder in the size scaling of material strength. Physical Review Letters 100, 055502 (2008).

[72] C. Chen, Z. Wang, Z. Suo, Flaw sensitivity of highly stretchable materials. Extreme Mechanics Letters 10, 50-57 (2017).

[73] C. Yang, T. Yin, Z. Suo, Polyacrylamide hydrogels. I. Network imperfection. Journal of the Mechanics and Physics of Solids 131, 43-55 (2019).

[74] S. Fakhouri, S. B. Hutchens, A. J. Crosby, Puncture mechanics of soft solids. Soft Matter 11, 4723-4730 (2015).

[75] V. Sorichetti, A. Ninarello, J. M. Ruiz-Franco, V. Hugouvieux, W. Kob, E. Zaccarelli, L. Rovigatti, Effect of Chain Polydispersity on the Elasticity of Disordered Polymer Networks. Macromolecules 54, 3769-3779 (2021).

[76] M. Stratigaki, R. Göstl, Methods for Exerting and Sensing Force in Polymer Materials Using Mechanophores. ChemPlusChem 85, 1095-1103 (2020).

[77] S. Sugita, T. Matsumoto, Local distribution of collagen fibers determines crack initiation site and its propagation direction during aortic rupture. Biomechanics and Modeling in Mechanobiology 17, 577-587 (2018). 



\section{Summary}

Fracture of materials is both undesirable and unavoidable; therefore, there is great interest in methods to either forecast or control the fracture process of a material. To develop these methods, a deep understanding of the microscopic fracture process is needed covering the entire process from the scission of the first atomic bond to catastrophic failure of the entire material. In this thesis we study the microscopic fracture processes in soft network materials, ranging from elastomers, like rubber, to biological fibre networks, like collagen. Due to their disordered network structure, these materials can undergo large deformations prior to the damage accumulation process, in contrast to stiff materials, like concrete, where deformation is negligible. Here we investigate the implications of these large deformations on the elastic response and fracture process of soft network materials. Specifically, we investigate the role of the network structure on elasticity and fracture via highly coarse grained models, wherein the network structure plays a central role. To cover the whole range of soft network materials ranging from soft brittle to soft ductile, we model both single network materials and composite networks materials where two or more networks are combined.

In Chapter 2 we introduce the simplest model to study fracture in networks that can undergo large deformations: the athermal central-force elastic model. In this two-dimensional model the network structure is simplified to a graph consisting of linear springs and nodes and its equilibrium configuration is found by an energy minimization procedure. The only elastic contributions in this networks come from the elements, however, rearrangements at the network level have a significant impact on the mechanical response. These rearrangements, and thus the elastic response, are governed by the rigidity of the network which depends on both the network topology and the applied deformation, and can result in highly heterogeneous distributions in stress throughout the network as well as the formation of force chains. We find that also the fracture response of these networks is controlled by rigidity. Furthermore, we observe a distinct dependence of the fracture behaviour on system size both at and after the strain at maximum strength, a common phenomenon in fracture. Finally, we use our model to explain the role of network structure in the fracture response of disordered collagen networks, revealing that the athermal central-force elastic model can be a helpful tool in understanding the microscopic fracture response of real soft network materials.

In the athermal model the effect of thermal fluctuations and other time related processes, such as relaxation, are neglected. However, many soft network materials 
are actually highly sensitive to thermal fluctuations. To study the effect of these fluctuations we embed our central-force elastic model in a Langevin dynamics environment in Chapter 3, introducing both thermal fluctuations and an implicit solvent. We confirm the picture that thermal fluctuations act as a stabilizing field, providing rigidity prior to the critical strain. We find that the network undergoes significant non-affine structural rearrangements around the critical strain to minimize the stress, with additional strong non-affine thermal fluctuations. As a result, the fracture process becomes a function of both rigidity and temperature, with a distinct coupling around the isostatic point. We find that temperature has two seemingly opposing effects on network fracture. The stabilizing field homogenizes stress with respect to the athermal simulation. However, the stochastic nature of the thermal fluctuations also allows rupture of bonds that are on average not overstretched, which can be understood as a destabilizing effect.

In Chapter 4 we shift our attention to composite networks, by investigating the linear and non-linear response of collagen networks embedded in a matrix of crosslinked hyaluronan polysacharrides, the two main component of the extracellular matrix, the network structure that supports the cells in our body. In this composite material there are two stabilizing fields that affect the linear response: bending rigidity of the collagen fibres and the elasticity of the hyaluronan matrix. Both these stabilizing fields resist the non-affine deformations that arise naturally in approaching the critical strain. Moreover, the coupling between the two stabilizing fields results in a enhancement in the linear modulus due to a competition between their preferred modes of local deformation. An intriguing experimental finding in this research is that upon crosslinking the hyaluronan, a negative normal stress arises, indicative of a tendency to compress. We find that the hyaluronan network pulls on the collagen fibres, which causes a delay in the onset of strain-stiffening. We have been able to capture both the enhanced linear modulus and the delayed strain stiffening by expanding the central-force elastic model, describing the composite as a subisostatic network with bending interactions between neighbouring bonds, representing the collagen network, which is coupled to a homogeneous soft network, representing the hyaluronan matrix. Furthermore, we showed that the mechanical response of this composite can be captured in a two parameter network model that allowed us to map our model on the experimental data demonstrating that the influence of the matrix on the mechanical response can be interpreted as two independent processes, damping of non-affine fluctuations affects the linear response, while pre-stress shifts the non-linear response.

In Chapter 5 we shift our focus from the elastic response to the fracture response of double networks, inspired by the significantly enhanced fracture response found in double network elastomers, hydrogels, and macroscopic materials. All these 
materials show that the response of an initially brittle network, also called the sacrificial network, can be shifted to a seemingly ductile response by embedding it in a significantly softer matrix network. We explore this transition from brittle to ductile behaviour by expanding upon the idea that the location of the brittle-toductile transition is governed by a force balance between the two networks. We locate the transition from brittle to ductile in a two-spring model, a multi-spring model and a random spring network model (another variant of the central-force elastic model) and find that in all models the location of the brittle-to-ductile transition can be predicted even when disorder is included in the latter two models. A detailed study of the network model reveals that also the development in the microscopic fracture response can be understood with respect to this brittle-to-ductile transition.

In Chapter 6 we also study the fracture response of a double network via a coarse grained model, but now we use a three-dimensional model that specifically targets polymer double networks. We explicitly model the polymers as a string of particles, so that each bond represents a Kuhn length. Furthermore, the model contains excluded volume interactions, so that topological constraints will arise between different polymer chains. Our network generation procedure is based on a consecutive polymerization process used to generate double networks in elastomers by swelling of the sacrificial network with the matrix monomer. The resulting networks have an exponential distribution in polymer length and can only interact via topological constraints. We find that our networks behave similar to the experimental systems and we can even rescale the initial mechanical response following a procedure proposed in literature for elastomers and hydrogels. We find that the damage response of our networks takes place in two steps. At first bond scission is governed by the first network, while after the yield strain interactions between the two networks dominate bond scission. This fracture mechanism, and the associated evolution of microscopic damage deviates significantly from the affine predictions for the damage response, where network structure is not considered. Overall this research demonstrates that redistribution of stress over the network plays an important role in the damage response of polymer networks.

In the general discussion we reflect on our findings and present a microscopic picture of fracture in soft network materials. Furthermore, we discuss a strategy to explore fracture processes that span a range of time scales, such as delayed fracture. Finally we discuss our findings in the context of continuum elasticity and provide an outlook for future research into fracture of soft network materials. 


\section{List of publications}

\section{This thesis}

- Federica Burla*, Justin Tauber*, Simone Dussi, Jasper van der Gucht and Gijsje H. Koenderink, 'Stress management in composite biopolymer networks', Nature Physics 15, 549-553 (2019). (Chapter 4)

- Simone Dussi, Justin Tauber and Jasper van der Gucht, 'Athermal fracture of elastic networks: how rigidity challenges the unavoidable size-induced brittleness', Physical Review Letters 124, 018002 (2019). (Chapter 2)

- Federica Burla*, Simone Dussi*, Cristina Martinez-Torres, Justin Tauber, Jasper van der Gucht and Gijsje H. Koenderink, 'Connectivity and plasticity determine collagen network fracture', Proceedings of the National Academy of Sciences 117, 8326-8334 (2020). (Chapter 2)

- Justin Tauber, Simone Dussi and Jasper van der Gucht, 'Microscopic insights into the failure of double networks', Physical Review Materials 4, 063603 (2020). (Chapter 5)

- Justin Tauber, Aimeé R. Kok, Jasper van der Gucht and Simone Dussi, 'The role of temperature in the rigidity-controlled fracture of elastic networks', Soft Matter 16, 9975-9985 (2020). (Chapter 3)

- Justin Tauber, Lorenzo Rovigatti, Simone Dussi and Jasper van der Gucht, 'Sharing the load: stress redistribution governs fracture of polymer double networks', Macromolecules, accepted (2021). (Chapter 6)

- Justin Tauber, Jasper van der Gucht and Simone Dussi, 'Stretchy and disordered: Towards understanding fracture in soft network materials via mesoscopic computer simulations', In preparation.

\section{Other work}

- Justin Tauber*, Ruben Higler*, and Joris Sprakel, 'Anomalous dynamics of interstitial dopants in soft crystals', Proceedings of the National Academy of Sciences 113, 13660-13665 (2016).

\footnotetext{
* These authors contributed equally to this work.
} 


\section{Acknowledgements}

In this thesis you have read how soft network materials deal with stress: by sharing the load. In the past few years I have experienced that the scientific community works in a similar way. In contrast to what the romantic depiction of scientists suggests, science is not an individual endeavour; as a PhD candidate you get to interact with many other scientists, sharing thoughts and experiences, both scientific and non-scientific. It is this sense of community that has made the monumental task of writing a thesis an overseeable journey and so much more enjoyable.

Jasper, thank you for offering me the opportunity to work within the SOFTBREAK project and welcoming me at PCC. Upon hearing about the project I had no clue about fracture of polymer networks, and even now I feel like we have only touched upon the beginnings of a microscopic understanding of fracture in these materials. I think this lack of knowledge - at least on my part - is exactly what intrigued me about this project. I am thankful for the freedom you provided me with in studying this topic. I admire your calm nature and your healthy habit to pay more attention to what goes well than what goes wrong. You have a way of formulating complex thoughts in a simple manner, which often helped me to reach a deeper understanding of the problems we were working on.

Simone, it was on my first day at PCC as a PhD candidate I heard you were working as a postdoc for the SOFTBREAK project and that you would be my daily supervisor. Since that moment you have been instrumental in every part of my thesis. We have discussed the ins and outs of network elasticity and fracture over and over again, refining our research questions along the way. I was very lucky to have two supervisors with such bright minds. Our work meetings were often exiting and filled with new insights, leaving me inspired, but also quite exhausted. I will be forever grateful that you decided to keep supervising me even after your postdoc at PCC was finished. I am almost certain that I would have lost myself in a labyrinth of details without your gentle reminders to stay focused on the main message.

During my PhD I had the oppertunity to work together with several excellent scientists from outside Wageningen. Federica and Gijsje, it is the dream of any scientist to be presented with consistent, but puzzling data and this is exactly what you showed us at the start of my PhD trajectory. It was a true joy to figure out this puzzle together in our collaborative effort. I was always amazed by the creativity of your experiments and your writing. Lorenzo, I enjoyed your visit in Wageningen and I am greatful for the effort you put in the project that followed. I 
learned a great deal from you about modelling polymers during our discussions. Federico, Julien, Tibor, you radiate energy when you talk about your experiments. Even though we haven't met each other in person (yet), one of our meetings even spanned three different time zones, we already did some cool work and I am looking forward to finishing it soon.

I am especially thankful towards the BSc and MSc thesis students that decided to work with me on this project and dealt with the continuous evolution of our research questions. Hans, during your entire thesis you seemed to have everything under control. It was fun working with you. Pieter, your attention for detail is noteworthy as well as your ability to ask the right questions. Aimeé, I appreciate your sense of humour. You do not only show a genuine enthusiasm for theory and simulations, but you are also good at it. Good luck in the future to all of you.

Many thanks to the SOFTBREAK team. Sven, Raoul and Simone, I especially remember our trip to the conference in Rolduc as a nice experience. Jose, although you joined the project at the end, we had many wonderful scientific discussions and even started working on a few projects together. I hope we can continue this effort.

Thank you Mara and Leonie for your genuine interest in my well-being and all the help with administrative tasks. Especially thanks to Leonie for helping out at the last moment with the submission of my thesis. Remco, you were of great help when I needed to do maintenance on the computers. Peter, Frans, René, Lennart, Dirk, Bart, I had the opportunity to help you with several courses. I learned a great deal from you about teaching chemistry and physics. Also thanks to Frans, Joshua and Renko for stimulating discussions at the simulation meetings and Joris and Tom for their advice on navigating through the rough landscape that is the academic world.

My office mates, it has been a pleasure sharing the office with all of you. Qimeng, we have been desk neighbours most of my PhD, and consequently we have discussed all kinds of things from our PhD struggles to food. I am glad I got to know you. Hanne, you're a kind-hearted person and your passion for science is contagious. Your thoughts are always crystal clear, which made our (scientific) discussions very enjoyable. Also, thank you for discussing my propositions with me. Fabiola, you are a kind and warm person. Dana, your lively spirit can instantly brighten up my day. Preeti, you are a hard worker with a lot of energy. I think we were a good team when we managed the group meeting together.

All my colleagues, thank you for the great atmosphere I experienced at PCC and your willingness to share stories about science as well as other matters during coffee breaks, planned and non-planned lunches, spontaneous evening meals, game nights, and $\mathrm{PhD} /$ postdoc weekends. I wish all of you the best of luck with 
either your projects at PCC or any other endeavours you embarked on in the meantime. Jochem and Raoul, having close study friends as close colleagues, made the transition to the $\mathrm{PhD}$ incredibly smooth and significantly increased my consumption of chocolate muffins and spare ribs. Ruben, there was never a dull moment during our lunch break conversations. Aljosha, your love of nature, literature and music is inspiring. Joanne, it was great to have you as a simulation buddy. Without your help I would have never even tried to assemble a computer cluster. Martijn, even though your visits were not always timely, I always enjoyed our discussions. I am also very happy that with your enthusiasm we could revitalize the simulation meeting. Jessica, your intriguing scientific work makes the world a more colourful place. I will always remember the splendid time we had in Edinburgh sipping whisky in the 'Auld Hoose'. Raisa, visiting conferences with you is always great fun. Sophie, although you joined PCC only in the last months of my PhD, your boundless enthusiasm was exactly what I needed to remind me of why I started doing a $\mathrm{PhD}$ in the first place. Keep up the spirit! Dana, Jochem, Martijn, Niek, Sophie, Chandan, Preeti and Akankshya, thank you for joining the online friday afternoon lab drinks, it really meant a lot to me.

Hoe geweldig spannend wetenschappelijk onderzoek ook kan zijn, er zijn nog genoeg andere dingen in de wereld om te ontdekken. Ik ben mijn vrienden en familie dan ook enorm dankbaar voor de nodige afleiding in de afgelopen jaren. Hanna, Jochem, Iris, Kate, Oberon en Robin, in welke combinatie we elkaar ook tegen komen, het is altijd gezellig. jullie staan altijd klaar om de hoogtepunten te vieren en de dalen in perspectief te plaatsen. Oberon en Sander, ik had onze (provincie)grensoverschrijdende tripjes vol droge humor zeker niet willen missen. Roos, als we samen koken en thee drinken wordt ik meteen getransporteerd naar onze zorgeloze studententijd op de Walstraat.

Mario, Karin, Koen, Merel. Bedankt voor jullie belangstelling in mijn onderzoek. Het "boekje" is nu echt af.

Opa en Oma, jullie vinden altijd wel een reden om weer uiteten te gaan met de familie, ik kan daar enorm van genieten.

Pap, Mam en Sherida. Bedankt voor alle etentjes, tripjes en natuurlijk de hulp bij het klussen. Doorzettingsvermogen is een van de voorwaarden voor het doen van wetenschappelijk onderzoek; dit is iets wat ik zeker van jullie heb geleerd.

Hanna, van iedereen heb je deze thesis het meest van dichtbij meegemaakt; zeker tijdens het laatste anderhalf jaar toen je onvrijwillig mijn enige kantoorgenoot werd. Ik ben enorm dankbaar dat ik deze tijd met jou heb mogen doorbrengen en hoop dat nog vele jaren te blijven doen. Bedankt voor alles! 


\section{About the author}

Justin Tauber was born on 21 Juli 1993 in Haarlem, the Netherlands. After receiving his high school diploma from the Stedelijk Gymnasium in Haarlem, he went on to study Molecular Life Sciences at Wageningen University, specializing in soft matter physics, and obtaining his MSc degree cum laude. As part of his master studies Justin conducted research for his MSc thesis at the Laboratory of Physical Chemistry and Soft Matter of Wageningen University, under the supervision of Dr. Ruben Higler and Prof. Joris Sprakel, using Brownian dynamics simulations to study the dynamics of interstitial dopants in soft colloidal crystals; for this work, Justin received the UFW Thesis award 2016. Subsequently, he visited the Soft Matter Engineering Group from Prof. Thomas Angelini at the University of Florida (United States), for an internship on 3D printing of human cells and collagen. In 2017, Justin started pursuing his PhD at the Laboratory of Physical Chemistry and Soft Matter of Wageningen University, studying the elasticity and fracture of soft network materials under the supervision of Dr. Simone Dussi and Prof. Jasper van der Gucht. This thesis is the result of this endeavor. In August 2021 Justin started a project in the Food Process Engineering Group at Wageningen University, under supervision of Prof. Karin Schroën, to investigate the potential of spontaneous emulsification in microfluidic devices for the production of micron sized silica particles. Justin plans to start a postdoc position abroad at the beginning of 2022. 


\section{Overview of completed training activities}

\section{Discipline-specific activities}

- Dutch Soft Matter Meeting, Delft (TU Delft 2017)

- Ameland Summer School Smart Materials, Ameland (4TU.HTM, 2017) ${ }^{\dagger}$

- Dutch Soft Matter Meeting, Enschede (TU Twente, 2017)

- Han Sur Lesse Winter School, Han Sur Lesse, Belgium (PCC/WUR, 2018)

- DYFP, Rolduc (TU/e 2018)

- Dutch Polymer Days, Lunteren (PTN 2018)

- 4TU.HTM Dutch Materials Day, Utrecht (4TU.HTM, 2018) ${ }^{\dagger}$

- Dutch Soft Matter Meeting, VU (VU, 2018)

- Physics@Veldhoven, Veldhoven (NWO,2019)

- Han Sur Lesse Winter School, Han Sur Lesse, Belgium (PCC/WUR, 2019)

- Dutch Polymer Days, Lunteren (PTN, 2019) ${ }^{\dagger}$

- International Soft Matter Conference, Edinburgh, U.K. (University of Edinburgh, 2019) ${ }^{\ddagger}$

- Physics@Veldhoven, Veldhoven (NWO, 2020) ${ }^{\dagger}$

- MRS Fall Meeting 2020, Boston/online, U.S.A. (MRS, 2020)

- Physics@Veldhoven, Veldhoven/online $(\mathrm{NWO}, 2021)^{\ddagger}$

\section{General courses}

- Scientific Artwork - vector graphics and images, Wageningen (WGS, 2017)

- VLAG PhD week, Baarlo (VLAG, 2017)

- Research Data Management, Wageningen (WGS, 2018)

- Start to supervise BSc \& MSc thesis students, Wageningen (WGS, 2018)

- Supervising BSc \& MSc thesis students, Wageningen (WGS, 2019)

- Scientific Writing, Wageningen (Wageningen Into Languages, 2019)

- Reviewing a scientific manuscript, Wageningen (WGS, 2019)

- Infographics and Iconography, Wageningen, (WGS, 2019)

- Critical Thinking and Argumentation, Wageningen (WGS, 2019)

- Career Assessment, Wageningen (WGS, 2019) 


\section{Other activities}

- Preparation of research proposal, Wageningen (PCC, 2017)

- Group meetings and colloquia, Wageningen (PCC, 2017-2021)

- Simulation meetings, Wageningen (PCC, 2017-2021)

- Mechanochemistry meetings, Wageningen (PCC, 2017-2019)

- Literature club, Wageningen (PCC, 2017-2021)

${ }^{\dagger}$ poster presentation

$\ddagger$ oral presentation 

This project has received funding from the European Research Council (ERC) under the European Union's Horizon 2020 research and innovation programme (grant agreement n'682782)

Printed by ProefschriftMaken || www.proefschriftmaken.nl 



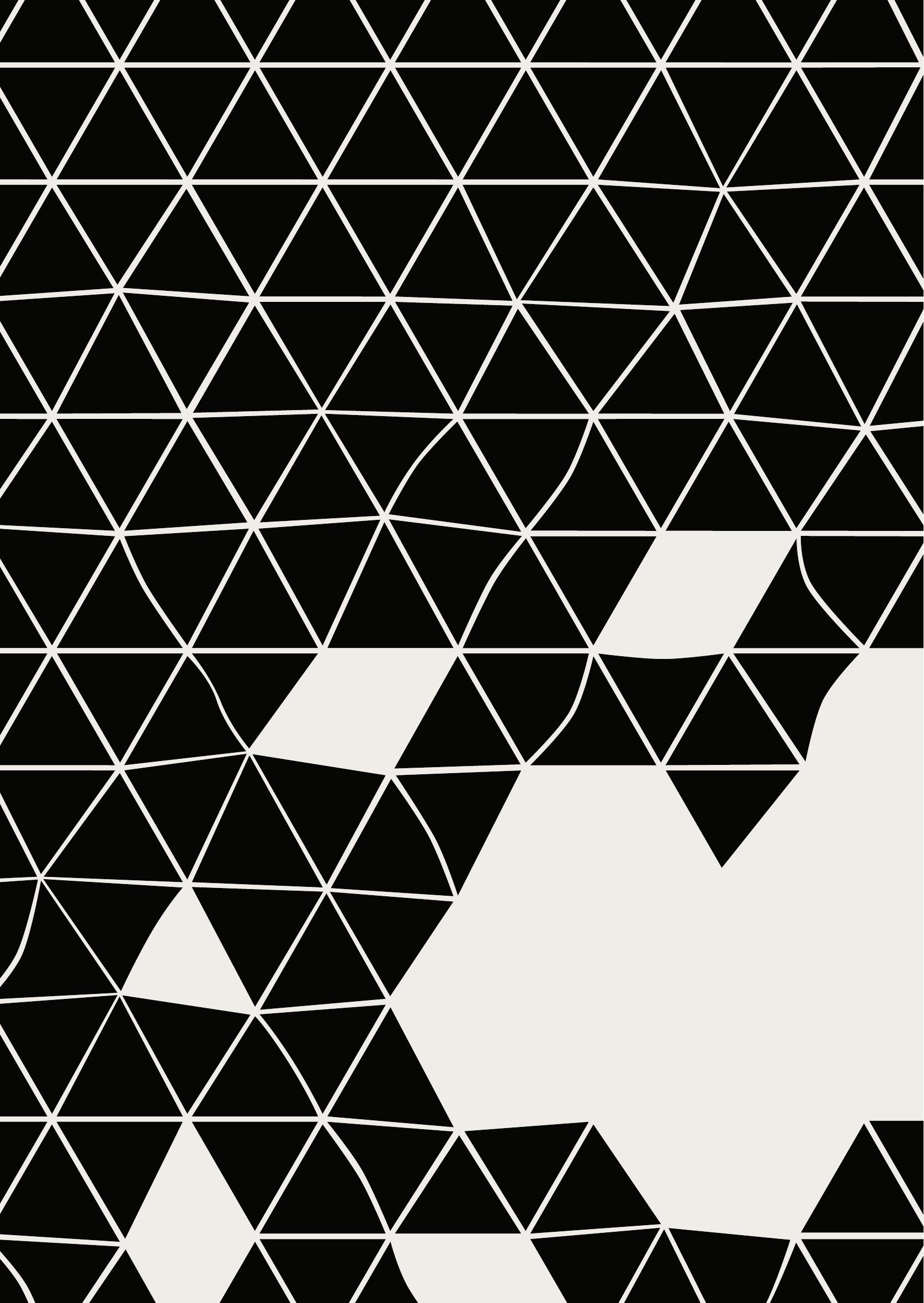

